\title{
Coordination and gapping in Catalan Sign Language (LSC)
}

Giorgia Zorzi

TESI DOCTORAL UPF / 2018

DIRECTOR DE LA TESI

Dr. Josep Quer

DEPARTAMENT DE TRADUCCIÓ I CIENCIES DEL LLENGUATGE

\begin{tabular}{|l|l}
$\mathbf{u} p f$. & $\begin{array}{l}\text { Universitat } \\
\text { Pompeu Fabra } \\
\text { Barcelona }\end{array}$
\end{tabular} 
To my sister and my parents

"your heart is a muscle the size of your fist. keep on loving. keep on fighting. and hold on, and hold on, hold on for your life."

Ramshackle Glory 


\section{Acknowledgements}

I would like to sincerely thank my supervisor Josep Quer for giving me the chance to work on Catalan Sign Language (LSC) in a very special place where I met amazing people and learnt new languages, among which Catalan became one of my favorites. I also want to thank him for the support and for always being available to look at new LSC data and discuss them together.

Among the people I was so lucky to meet at UPF, I want to first thank very much the LSC Lab for the activities, the fun LSC lunches and the constant support for everything: Gemma Barberà, Alexandra Navarrete-González, Raquel Veiga Busto, Eréndira Cervantes Carreto, Jordina Sánchez Amat, Sara Cañas Peña, Xavi (Francisco Javier Alvarez), Ryan Simoens, Sílvia Gabarró-Lopez, Celia Alba, Juana Roselló, Noelia Hernández; a very special thank goes to Delfina Aliaga Emeterio and Santi Frigola Segimon, I am very grateful I could work with you both. Thank you for your patience when I mixed languages, signing or writing. Also thank you for having been my LSC teachers. I also want to thank my office mates over the years: Kata Wohlmuth, Iris Hübscher, Josep Ausensi, Veronika Richtarcikova, Santiago Gonzalez, Olga Kushch, Alfonso Igualada and Florence Baills and also Anderson Almeida, even if for only few months, but it was so nice to have you there, tea buddy. A special thanks to Núria Esteve-Gibert for the patience with the inprogress learning Catalan and the biggest hugs every time I see you,

to Toni Bassaganyas Bars for being an amazing office buddy and friend, to Alexandra Navarrete for making me so excited about 
contrast, to Raquel Veiga Busto for always adding a nice energy anywhere you are, to Eréndira Cervantes Carreto for the constant support and to Antonia Tovar for the fun times together and for being the best teaching buddy. From the GLiF group I also want to thank Louise McNally, Laia Mayol, Gemma Boleda, Cristina RealPuigdollers, Kristina Gulordava, Carina Silberer, Julie Hunter, Álex Alsina, Enric Vallduví, Wolfram Hinzen, Josep Maria Fontana, Gabriel Sevilla, Ernesto Cota, Chenjie Yuan, Eugenio Vigo, Matthijs Westera and among the PhD students Fengrong Yang, Ionut Sorodoc, Laura Aina and Dominika Slusna. From the former members, a special thank goes to Paula Menendez Benito for the walks and super nice chats in Gràcia.

During my PhD I also had the chance to spend four months as a visiting student at UMass Amherst. I really want to thank Vincent Homer for giving me this opportunity and for always being supportive. A big thank also to Kyle Johnson for supporting me in my path in discovering the beauty of gapping, either meeting at UMass or later at summer schools or conferences. Among the people I had the chance to meet, I want to thank in particular Kiyomi Kusumoto, Deniz Ozyildiz, Lisa Hofmann, Jon Ander Mendia, Ekaterina Vostrikova, Rodica Ivan, Thuy Bui, Jyoti Iyer, Rong Yin, Sakshi Bhatia, Alexander Göbel, Petr Kusliy, Nicholas LaCara, Chris Hammerly, Rajesh Bhatt, Seth Cable, Brian Dillon, Jeremy Hartman and Barbara Partee for making my time at UMass an amazing experience. A big thank also goes to Manda and Corey for showing up at my first ASL meeting and becoming my ASL buddies. 
I also had the chance to collaborate with the Sign-Hub project so I want to thank a lot Josep Quer, again, and also Caterina Donati, Carlo Cecchetto, Naama Freedman, Lara Mantovan, Alessandra Checchetto, Beatrice Giustolisi, Chiara Annucci, Valentina Aristodemo. A very special thank goes to Jordina Sánchez Amat, I love working with you, you are an amazing person!

I want to thank the people who gave me precious feedback and a lot of support at conferences and workshops. I want to thank Carlo Geraci, Carlo Cecchetto, Elena Koulidobrova, Kyle Johnson, Caroline Féry, Caterina Donati, Vadim Kimmelman, Roland Pfau, Jason Merchant, Philippe Schlenker, Asli Ozkul, Yasutada Sudo, Elena Castroviejo Miró, Jan-Wouter Zwart, Gillian Ramchand, Laura Vela-Plo, Charlotte Hauser, Kadir Gökgöz, Kate Davidson, Annika Herrmann, Matic Pavlic, Derya Nuhbalaoglu and Anne Wienholz. With them, also the interpreters that made conferences more fun: Christopher Stone, Oliver Pouliot and Lissa Zeviar.

I am also grateful to my MA supervisor Carlo Geraci, I would have not come to Barcelona to do a $\mathrm{PhD}$ without the introduction to the sign language research world from him, thank you.

This research would not have been possible without the funding by the Spanish Ministry of Economy and Competitiveness (MINECO \& FEDER: ClauseCombi2 FFI2012-36238, BES2013062848 \& GramRefLSC FFI2015-68594-P), by the Govern de la Generalitat de Catalunya (2014 SGR 698), BES2013062848 2017 SGR 1478 and SIGN-HUB project, which has received funding from the European Union's Horizon 2020 research and innovation programme under grant agreement No 693349. 
I am also very grateful for meeting two special people who I had the chance to see almost every day and with whom I shared a lot of quality time together. Many, many thanks to Iris Hübscher and Kata Wohlmuth for becoming not just working buddies but also special friends, always there for me, also in the darker times; not even all the cakes, stroopwafels, cigarette breaks and walks along the beach or at the park in the whole world could show my appreciation. Among the friends that were not at the university that I want to thank very much there is Babo (Borja), for always making me feel at home and be there for me... and therefore also to Irene, David, Adela, Joan, Ombra and Rubén. Thanks to Mike, for walking together to places where I would have never gone myself. Thanks also to Teresa Suñol for teaching me so many interesting things about bees and nature. A huge thank also goes to the friends that are very far but at the same time so close...to Lillino (Silvia) for always being there and comforting me anytime I need it; to Masha, thank you "gatto" for making Paris a place where I feel at home and to Hannah Lutzenberger, for being the most fun and smart signing person I have ever met.

Finally, and most importantly, to my family. To my parents, for supporting me during these many years of studying around the world and always being encouraging. Without you I probably would not have had the chance to end up doing a $\mathrm{PhD}$ in linguistics. To Daniel, for being there when I was not. And the biggest thank goes to my sister, Marika, who I cannot thank enough. Love you, sis.

Barcelona, 2018 


\section{Abstract}

This thesis gives a description and a syntactic analysis for coordination and gapping in conjunction in Catalan Sign Language (LSC) within the framework of Generative Grammar and Minimalism. Regarding coordination, Coordination Phrase (CoP) is proposed as the category for conjunctive, disjunctive and adversative coordination, assuming that the conjuncts are specifiers and complements of $\mathrm{CoP}$ in a right-branching coordination structure. The specific derivation for each types of coordination is then applied. As for gapping in conjunction, in LSC it shows similarities with VPellipsis, especially because it can appear also in subordination. Moreover, the availability of only distributed scope negation $(\neg \mathrm{A} \& \neg \mathrm{B})$ and the presence of contrastive topic and contrastive focus require a large coordination structure, that is, $\mathrm{CP}$ coordination. In order to derive gapping, I propose movement of the arguments to TopP and FocP followed by the deletion of TP at PF, with [E] feature in the head of FocP.

\section{Resum}

Aquesta tesi ofereix una descripció i una anàlisi sintàctica per a la coordinació i el "gapping" en coordinació conjuntiva en llengua de signes catalana (LSC), dins el marc generativista i minimista. Pel que fa a la coordinació, la categoria sintàctica que es proposa és "Coordination Phrase" (CoP) per a la coordinació conjuntiva, 
disjuntiva i adversativa. A l'estructura, ramificada a la dreta, els constituents de la conjunció són especificadors i complements de CoP. La derivació per a cada tipus de coordinació s'aplica a partir d'aquest model. Pel que fa al "gapping", mostra proprietats similars a l'el-lipsi de SV (VP-ellipsis), sobretot perquè pot aparèixer en subordinació. A més, la l'existència només d'un abast distribuït de la negació $(\neg \mathrm{A} \& \neg \mathrm{B})$ i la presència de tòpic i focus contrastius mostra la necessitat de tenir una coordinació "àmplia" on els dos conjunts siguin CPs. En la derivació de "gapping", els arguments es mouen a TopP i FocP, seguits de l'eliminació del TP a PF, ambel tret [E] posicionat al nucli de FocP. 


\section{Table of contents}

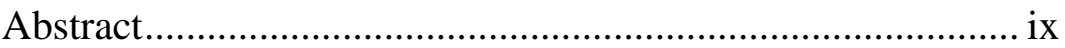

List of abbreviations .........................................................

Annotation conventions ..................................................... Xvi

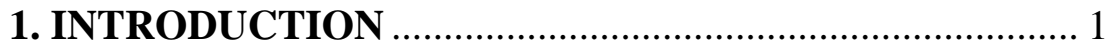

1.1 Introduction to Catalan Sign Language (LSC) ................... 6

1.2 Methodology........................................................................ 7

PART I: COORDINATION ..................................................... 11

\section{COORDINATION IN SPOKEN AND SIGN}

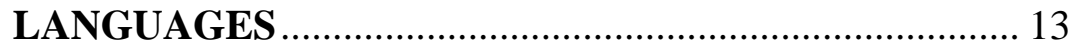

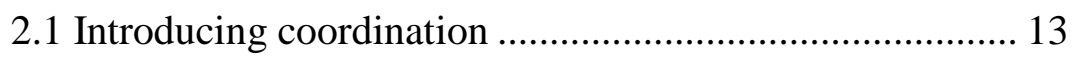

2.1.1 Types of coordination .............................................. 15

2.1.2 Asyndetic and syndetic coordination......................... 18

2.1.3 Position of the coordinator: head initial and head final

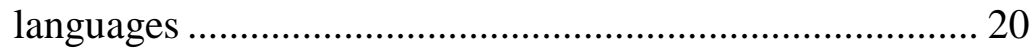

2.1.4 Symmetric and asymmetric coordination .................. 24

2.2. Syntax of coordination ................................................... 36

2.2.1. Asymmetries in coordination................................... 37

2.2.1.1 Directionality of coordination ............................ 42

2.2.2. What can be coordinated?......................................... 46

2.2.3. Syntactic properties: CSC and ATB ........................ 48

2.2.4 Towards a syntactic structure ................................... 52

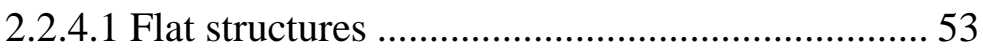

2.2.4.2 Non-flat structures ............................................. 56

2.2.4.2.1 Conjuncts are specifiers and complements of

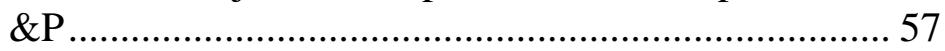

2.2.4.2.2 Each conjunct is the complement of its own

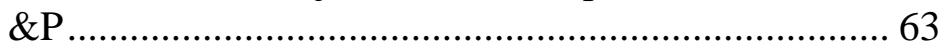

2.2.4.2.3 Conjuncts are adjuncts and coordinators are

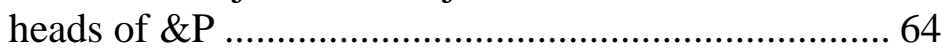

2.2.4.2.4 Conjuncts are heads but not of \&P .............. 65 
2.2.5 Disjunctive and adversative coordination................. 69

2.2.5.1 Disjunctive coordination ..................................... 69

2.2.5.2 Adversative coordination.................................. 72

2.2.6 Summary and conclusions ......................................... 78

2.3 Coordination in sign languages ..................................... 79

2.3.1 Types of coordination in sign language.................... 80

2.3.2 Expression of coordination in SLs: manual and non-

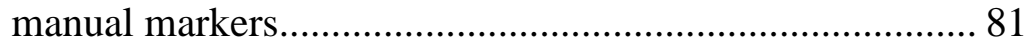

2.3.2.1 Conjunctive coordination .................................. 81

2.3.2.2 Disjunctive coordination .................................... 85

2.3.2.3 Adversative coordination..................................... 87

2.3.3 Tests to identify coordination (vs subordination)...... 89

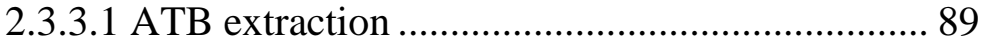

2.3.3.2 Gapping …..................................................... 91

2.3.3.3 Scope of yes/no questions and negation (in conjunction) …............................................................. 93

2.3.3.4 Distributional dependency test ........................... 95

2.3.3.5 Presence of prosodic boundaries ......................... 97

2.3.4 Conjunction (vs) disjunction: analysis ...................... 98

2.3.5 Summary and conclusions ...................................... 102

\section{COORDINATION IN CATALAN SIGN LANGUAGE}

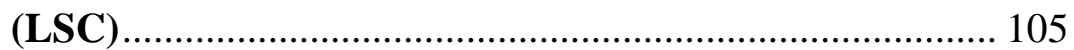

3.1 Expression of coordination in LSC …........................... 105

3.1.1 Conjunctive coordination ........................................ 107

3.1.1.1 NMMs for conjunctive coordination ................ 107

3.1.1.2 Manual markers for conjunction........................ 113

3.1.2 Disjunctive coordination ....................................... 125

3.1.2.1 NMMs for disjunctive coordination ................. 125

3.1.1.2 Manual markers for disjunctive coordination... 127

3.1.3 Adversative coordination........................................ 137

3.2 Properties of coordination in LSC .................................. 140 
3.2.1 What can you coordinate? ....................................... 140

3.2.2 Asymmetries in coordination.................................. 143

3.2.2.1 Semantic asymmetry....................................... 143

3.2.2.2 Syntactic asymmetry....................................... 148

3.2.3 Tests to identify coordination in LSC..................... 152

3.2.3.1 ATB extraction: wh-movement? ...................... 152

3.2.3.2 Scope of yes/no questions and negation (in

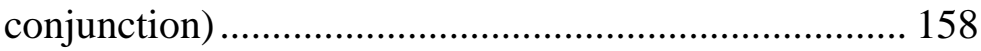

3.2.3.3 Distributional dependency test ......................... 162

3.2.3.4 Presence of prosodic boundaries ...................... 163

3.3 Analysis of coordination in LSC ................................... 165

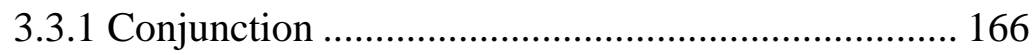

3.3.1.1 Coordination of simultaneous events ............... 175

3.3.1.2 List-buoys ......................................................... 186

3.3.2 Disjunction (vs conjunction) ..................................... 194

3.3.3 Adversative coordination........................................ 197

3.4 Remarks on directionality of coordination and other

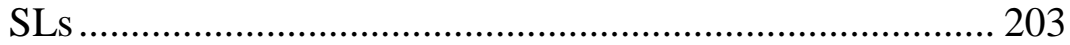

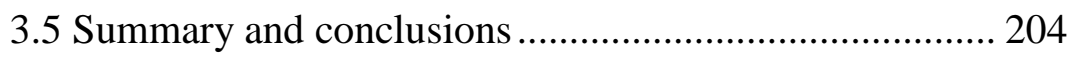

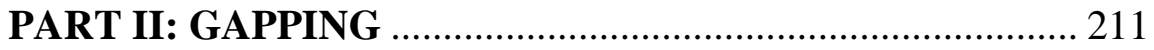

4. ELLIPSIS AND GAPPING .......................................... 213

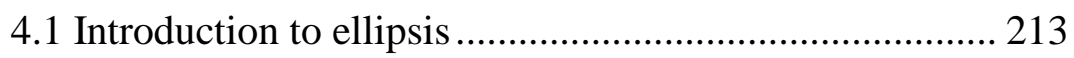

4.1.1 Analysis: different approaches ............................... 216

4.1.1.1 Non-structural approaches ............................... 216

4.1.1.2 Structural approaches ....................................... 218

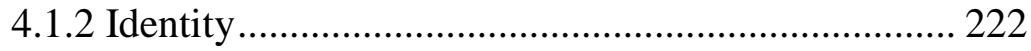

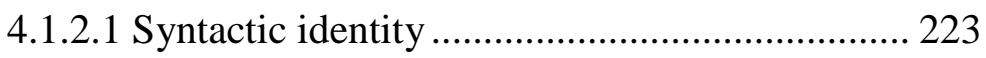

4.1.2.2 Semantic identity ............................................ 226

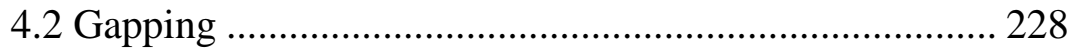

4.2.1 Properties of gapping ............................................ 229 
4.2.1.1 Gapping and information structure. 239

4.2.2 Approaches to gapping .......................................... 242

4.2.2.1 Small-conjunct coordination.............................. 243

4.2.2.2 Large-conjunct coordination.............................. 249

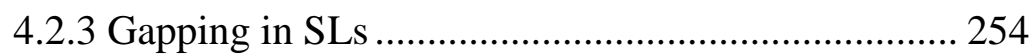

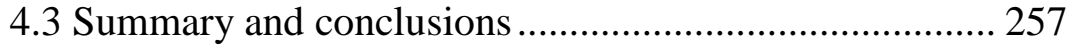

5. GAPPING IN CATALAN SIGN LANGUAGE.............. 259

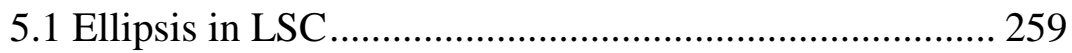

5.1.1 Agreement in LSC and classification of ellipsis ..... 262

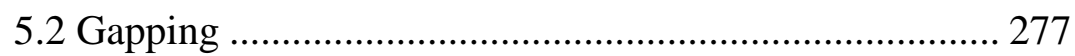

5.3 Discourse properties in gapping in LSC ........................ 288

5.3.1 NMMs of contrastive topic and contrastive focus... 289

5.4 Towards an analysis of gapping in LSC ........................ 300

5.4.1 Against small-conjunct coordination and ATB ....... 301

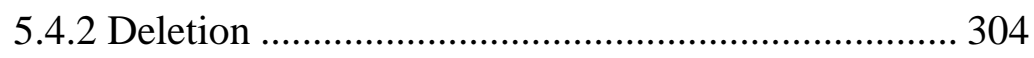

5.4.2.1 Evidence for structure in gapping..................... 305

5.4.2.2 What can you delete?...................................... 307

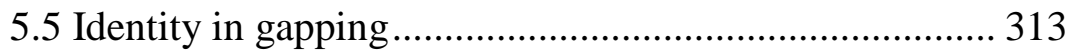

5.5.1 Identity in form: $\varphi$-features mismatch ..................... 314

5.5.1.1 Special cases of verbal CL and their realization in agreement and gapping ............................................... 343

5.5.2 Identity in meaning: adverb incorporation mismatch 348

5.6 Directionality of coordination and of gapping: a unified

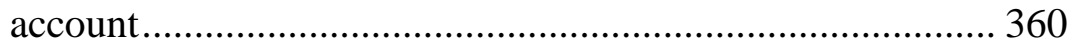

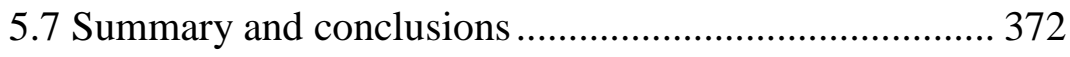

6. FINAL REMARKS AND FUTURE DIRECTIONS ....... 379

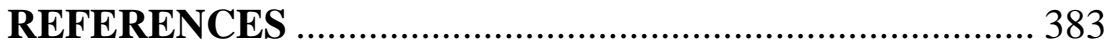




\section{List of abbreviations}

Abbreviations of sign languages

$\begin{array}{ll}\text { ASL } & \text { American Sign Language } \\ \text { Auslan } & \text { Australian Sign Language } \\ \text { BSL } & \text { British Sign Language } \\ \text { DGS } & \text { Deutsche Gebärdensprache, German Sign Language } \\ \text { FinSL } & \text { Finnish Sign Language } \\ \text { HKSL } & \text { Hong Kong Sign Language } \\ \text { LIS } & \text { Lingua dei Segni Italiana, Italian Sign Language } \\ \text { LSC } & \text { Llengua de signes catalana, Catalan Sign Language } \\ \text { LSE } & \text { Lengua de signos española, Spanish Sign Language } \\ \text { LSF } & \text { Langue des Signes Française, French Sign Language } \\ \text { NGT } & \text { Nederlandse Gebarentaal, Sign Language of the } \\ & \text { Netherlands } \\ \text { RSL } & \text { Russian Sign Language } \\ \text { TiD } & \text { Turkish Sign Language }\end{array}$




\section{Annotation conventions}

\section{General annotation}

$\begin{array}{ll}\text { FLOWER } & \text { Lexical sign } \\ \text { PERSON+++ } & \text { Reduplication of sign } \\ \text { N-A-D-I-A } & \text { Fingerspelled word } \\ \text { IX-1 } & \text { First person pronoun } \\ \text { IX-2 } & \text { Second person pronoun } \\ \text { IX-3 } & \text { Third (person) pronoun } \\ \text { IX-3i } & \text { Coreferential index } \\ \text { IX-1-POSS } & \text { Possessive pronoun (first person singular) } \\ \text { 1-GIVE-2 } & \text { Agreement verb inflected for first and second } \\ & \text { person singular meaning "I gave you" } \\ \text { 1AUX2 } & \text { Auxiliary inflected for first and second person } \\ \text { CL } & \text { Classifier } \\ \text { PICK-UP-CL } & \text { Verb expressed with a classifier } \\ \text { EAT-A-LOT } & \text { Verb expressed with a morphologically } \\ & \text { incorporated adverb meaning "eat a lot" } \\ \text { hd } & \text { Dominant hand } \\ \text { hd } & \text { Non-dominant hand (weak hand) } \\ \text { LIST2----- } & \text { Holding of a sign }\end{array}$

\section{Non-manual markings (NMMs)}

\begin{tabular}{|c|c|}
\hline re & Raised eyebrows \\
\hline $\mathrm{fe}$ & Furrowed eyebrows \\
\hline space contralateral & $\begin{array}{l}\text { Use of the contralateral side of the space } \\
\text { (notation with any kind of abbreviation } \\
\text { depending on the space available) }\end{array}$ \\
\hline space ipsilateral & $\begin{array}{l}\text { Use of the ipsilateral side of the space (notation } \\
\text { with any kind of abbreviation depending on the } \\
\text { space available) }\end{array}$ \\
\hline $\mathrm{bl}$ & Body lean \\
\hline
\end{tabular}




\begin{tabular}{ll}
$\frac{\mathrm{hl}}{\mathrm{lb}}$ & Head lean \\
$\frac{\mathrm{bbl}}{\mathrm{bs}}$ & Lean back \\
$\frac{\mathrm{hs}}{\mathrm{lcd}}$ & Backward body lean \\
\hline$\frac{\mathrm{hl}+\mathrm{bs} \text { ipsil. }}{\mathrm{hn}}$ & Body shift \\
\hline$\frac{\mathrm{shn}}{\mathrm{hth}}$ & Head shift \\
\hline $\mathrm{hthb}$ & Lips curved down \\
\hline $\mathrm{sq}$ & More than one NMMs appear together \\
\hline $\mathrm{hs}$ & Sead nod \\
\hline $\mathrm{eb}$ & Head hrust head nod \\
\hline $\mathrm{pc}$ & Head thrust back \\
\hline$[\mathrm{xxx}]$ & Squinted eyes \\
& Head shake (in combination with negation) \\
& Eye blink \\
& Puffed cheeks \\
& Lexical marker: a mouth gesture or mouthing \\
(silent articulation of a spoken word)
\end{tabular}





\section{INTRODUCTION}

Coordination and gapping are two phenomena that often come together. Coordination is present in all languages, even though it can be expressed in different ways, and it is found not just in spoken languages but also in sign languages (SLs). Through coordination it is also possible to realize ellipsis, which comes in different types, among which one is gapping. Unlike coordination, which already received some attention in SLs (HKSL by Tang and Lau (2012), ASL by Davidson (2011), FinSL by Jantunen (2015) and NGT by Legeland et al. (2018)), gapping is SLs is still an understudied phenomenon. Several authors presented data on ellipsis and outlined directions for a potential analysis, but no formal syntactic analysis has been proposed so far. Cecchetto et al. (2015) give an analysis for VP ellipsis in LIS, and Schlenker (2014) also presents data related to that for ASL and LSF. Some authors provide data on gapping, but without giving a thorough analysis (Liddell (1980) and Frazier \& Yosida (2012) for ASL, Jantunen (2013) for FinSL and Tang \& Lau (2012) for HKSL).

The goal of this thesis is to provide a thorough description and an exhaustive formal syntactic analysis for coordination and gapping in Catalan Sign Language (LSC), within the framework of Generative Grammar, and more specifically within Minimalism. For these understudied phenomena, it will be an important contribution from a syntactic point of view, in a way that was never undertaken before for a sign language. 
This work is divided in two parts: the first one focuses on coordination and the second one on gapping. In the first part, dedicated to coordination, I will be focusing mainly on sentential coordination, and I will present data on conjunctive, disjunctive and adversative coordination. Generally, the goal here is to provide an appropriate syntactic structure and in order to do that, I will answer the following questions: i) is there asymmetry between conjuncts?; ii) what categories can be coordinated?; iii) what are the properties of extraction in coordination?, and, finally, iv) what is the structure that represents coordination in syntax best and what is its directionality? The first two questions are important to determine the presence of an asymmetric syntactic structure, going against a flat one. As for the categories that can be coordinated when they are different, the fact that the conjuncts always need to have a category that saturates the verb shows that there is no preference in adopting the category of the first conjunct as the one for the whole coordination structure, contrary to what Zhang (2010) argues for spoken languages. This aspect supports the adoption of Coordination Phrase $(\mathrm{CoP})$ as syntactic category of coordination in LSC. It is also justified by the modality specific expression of coordination in SLs through the use of non-manual markings (NMMs) that makes this structure detectable since the beginning of the sentence. Questions iii) and iv), instead, are crucial in determining the properties of the structure itself and its directionality. Ross (1967) formulated the Coordination structure constraint (CSC), which states that it is not possible to extract from only one of the two conjuncts, but it is necessary to do it out of both for the same element, in an across-the-board (ATB) 
fashion. In LSC, though, this constraint does not apply. Considering this point in combination with the directionality of the structure, we will see that it can be a way to justify its ungrammaticality. Looking at the intonational grouping of the coordinator with respect to the conjuncts and following Zhang's (2010) generalization, the structure will be right-branching and the conjuncts specifiers and complements of CoP, following Munn (1987). For conjunction, particular attention will be given to the formalization of the expression of simultaneous events thought the modality specific "weak hand hold", for which a multidominant structure will be adopted, following Kimmelman (2017). As for disjunction, a more semantic approach will be used, integrating a quantificational account in the syntactic structure, following Davidson (2013). Both conjunction and disjunction are mainly expressed not with a manual marker, but through the use of non-manual markings (NMMs) that allow to distinguish these two structures. The presence of a universal or an existential quantifier scoping over the whole structure will be the element determining the presence of conjunction or disjunction, respectively. For the analysis of adversative coordination, I will consider the corrective and counterexpectational types, following Vicente's (2010) analysis. I will argue that corrective adversative coordination consists of clausal coordination plus deletion, and, in the case of counterexpectational adversative coordination, it is allowed for the conjuncts to be smaller than clauses. This analysis of coordination in LSC will be the first one to be extended to the three different types of coordination conjunctive, disjunctive and adversative - for a sign language. Moreover, the identified properties related to coordination, such as 
extraction and scope of negation, contribute to the cross-linguistic and cross-modal comparison between spoken and sign languages. These properties are also relevant when looking at ellipsis.

The second part of this thesis focuses on gapping and its properties and has the goal of providing a syntactic analysis for it. Gapping relates to coordination because it must be expressed through it. Interestingly, unlike many spoken languages, gapping in LSC can also appear in subordination. In addition, it does not respect another property that is very common for most of spoken languages, the No embedding constraint, which is usually used as a diagnostic for gapping in comparison to VP-ellipsis. The constraint does not apply to gapping in LSC. The aim of this section on gapping is, then, to answer the following questions: i) what are the properties of gapping in LSC?; ii) is gapping a unique type of ellipsis that can only appear in coordination and respects the No embedding constraint?; iii) what is the classification of the ellipsis types in LSC?, and, finally, with respect to the analysis, iv) is there structure in the ellipsis site in gapping?, and v) is there identity in form or in meaning between the antecedent and the gapped site?

Focusing on the properties of gapping, LSC shows a different pattern than spoken languages. Gapping in LSC can appear in subordination, as it is also the case for VP-ellipsis (VPE). The analysis for gapping will require large coordination structure, meaning $\mathrm{CP}$ coordination, due to the existence of only distributed scope negation $(\neg \mathrm{A} \& \neg \mathrm{B})$ and the presence of contrastive topic and contrastive focus. Moreover, the sensitivity to islands justifies the presence of deletion at PF, following Merchant (2001), with the E- 
feature placed in the head of FocP. VPE shows similar properties to gapping, but even if it targets a different portion of the structure, a unified account for gapping and VPE will be proposed. In both structures, as well, information structure plays a crucial role. The remnants in both structures are put in contrast and in gapping the external argument is marked as contrastive topic and the internal one as contrastive focus; the only remnant in VP-ellipsis will be marked as contrastive focus. Another factor to take into account in the analysis of gapping in LSC is the presence of $v$ to T movement of the verb. In the derivation of gapping, since it is the verb that is targeted, it is the whole TP that gets deleted. As for the identity relation between the antecedent and the gapped site, we will see, looking at $\varphi$-feature mismatch and adverb incorporation, that both identity in form and in meaning can be found. The data presented for gapping in LSC also help to classify the main types of ellipsis and justify a unified account at least for gapping and VPE, even though the parts of the structure affected are different.

Finally, the discussions about coordination and gapping are very much related when we look at their directionality. Despite LSC being a head-final language - and as such we would expect a leftbranching coordination structure -, gapping happens forward, realizing an SOV-SO order. The necessity of licensing the gap in the second conjunct leads to confirm the presence of a right-branching coordination structure. The ungrammaticality in extracting in ATB fashion, in having negation scoping over both conjuncts and in producing right-node raising (RNR) can be justified by the same means, too. As I will show, the directionality of gapping and that of 
coordination go together since it is the latter that determines the former cross-linguistically and cross-modally.

\subsection{Introduction to Catalan Sign Language (LSC)}

Sign languages (SLs) are natural languages used by deaf communities in the whole world. They are realized in the gesturalvisual modality, but despite this difference with spoken languages in the aural-oral modality, these two groups of languages have similar properties. In SLs phonology, morphology and syntax, semantics and pragmatics are also the core components of their grammars. In this thesis I will focus on Catalan Sign Language (LSC).

LSC is the sign language used by the deaf community in Catalonia (Spain) by around 25,000 signers among which are 12,000 deaf people, as estimated by the Catalan Federation for the Deaf. LSC, together with LSE, was recognized as a language of Spain in 2007 (Ley27/2007, October 23rd 2007) and its use in the public life was regulated in 2010 by the Catalan Parliament (Llei 17/2010, June 3rd 2010) after already including the right of using LSC in Catalonia in 2006 (Quer et al. 2010).

As other sign languages, because of their modality, LSC makes grammatical use of space and grammar is conveyed also by the use of non-manual markers (NMMs) that involve different facial expressions and movements of the body or of the head. NMMs are crucial in expressing, for example, complex structures such as polar questions and thanks to NMMs they can be distinguished from declarative sentences. Moreover, the spreading of NMMs is used also 
in LSC as a tool to detect the presence of syntactic structure, as for example in the case of $\mathrm{v}$ to $\mathrm{T}$ movement of the verb in combination with the NMMs for negation spreading over the verb. Moreover, NMMs are crucial when looking at prosody and the interaction with information structure notions like topic and focus. As for the use of space, as shown by Barberà (2012), spatial locations are also part of the grammar and each spatial plane contains its grammatical features, and, for instance, specificity is expressed in lower spatial locations.

Looking at word order, LSC is considered a head final language with SOV order that triggers a left-branching structure. The position of the subject, though, as in other SLs, is claimed to be on the specifier position of TP directed to the left (Pfau \& Quer 2007).

Finally, LSC, as other SLs and spoken languages, shows a wide range of syntactic structures, among which there are also modality specific ones such as role shift.

\subsection{Methodology}

The LSC data presented in this work were collected through data elicitation with two deaf LSC native signers, Delfina Aliaga Emeterio and Santi Frigola Segimon. Due to detailed features that were needed to be accounted for in both coordination and gapping, the existing LSC corpus, started in 2012 and in progress of annotation, would have not provided a representative sample of the potential of the language for these two structures.

Most of the elicitation sessions were conducted with both informants and the language used was LSC. The possibility of 
working the three together was very beneficial, because the two informants could discuss the data in LSC and provide each other with examples in native LSC.

The data elicitation happened in different ways, depending on the topic. The more common way to work was to provide glossed sentences in written Spanish, the written language both informants are more comfortable with, after giving a context signed in LSC. The glosses had the goal to make the informants produce the sentence requested and judge its grammaticality or ungrammaticality. There were also cases in which the first input for the sentences was directly provided in LSC. Depending on the topic, pictures were also used. The data discussed was filmed, turned into mp4 using iMovie and annotated with ELAN, a software developed at the Max Planck Institute $^{1}$ (Lausberg \& Sloetjes 2009). In the following sessions, the filmed sentences were showed again to the informants in order to confirm their judgments about them or to clarify further aspects. For each session, the videos recorded were collected together in one video and then numbered using ELAN. In this thesis, the LSC examples will have a number close to them, e.g., "80.10". The first number corresponds to the session and the second to the sentence. An edited version with subtitles for the LSC examples is provided in the text when close to the example there is a camera symbol (nat will direct the reader to an on-line folder if clicked on. Despite the restricted number of LSC experts the data were collected with, the

\footnotetext{
${ }^{1}$ ELAN (Version 4.8.1) [Computer software]. Nijmegen: Max Planck Institute for Psycholinguistics. Retrieved from https://tla.mpi.nl/tools/tla-tools/elan/
} 
1. Introduction

possibility of asking judgments about the same sentences several times guaranteed a quantitative and qualitative analysis. 


\section{PART I: COORDINATION}




\section{COORDINATION IN SPOKEN AND SIGN LANGUAGES}

In this chapter we will present the background on coordination in spoken and signed languages. We will describe this phenomenon and we will go through the analyses that have been proposed in the literature. In 2.1 we will introduce the main types of coordination: conjunctive, disjunctive and adversative. In 2.2 we will illustrate the syntactic properties of each type and then go through the syntactic analyses proposed in the literature. We will first address general questions about coordination and then enter the details of the syntactic analysis for conjunctive, disjunctive and adversative coordination. In 2.3, we will describe the properties of coordination in SLs. We will illustrate the modality specific strategies used to express it and the tests used to identify coordination in contrast to subordination. Despite the limited amount of formal analyses on coordination, we will present the ones proposed in the literature for conjunction and disjunction.

\subsection{Introducing coordination}

Every language shows the use of coordination. Haspelmath (2007) defines coordination as a syntactic construction in which two or more units of the same type are combined into a larger unit and still have the same semantic relations with other surrounding elements. He also points out that it is possible to coordinate words (1a), phrases (1b), subordinate clauses (1c) and sentences (1d). 
(1) a. My husband supports and adores Juventus Turin.

b. My uncle or your in-laws or the neighbors will come to visit us.

c. I realize that you were right and that I was mistaken.

d. The pope dissolved the Jesuit order, and all the Indian missions were abandoned.

(Haspelmath 2007: 1)

The parallelism between the two conjuncts, the two units coordinated, leads to a syntactic and semantic symmetry in coordination, like in the examples in (1). However, many researchers noticed that it is possible to combine different categories, as Sag et al. (1985: 143) for (2a) and Munn (1993: 80) for (2b) did, among others. These are cases of asymmetric coordination.
a. Jermaine is [AP boring] and [NP a fool].
b. You can depend [PP on my assistant] and [CP that he will be on time].

In this chapter we will first look into the different types of coordination trying to give an overview of the properties and functions of this structure across languages, mainly focusing on English. Afterwards, we will go through the syntactic proposals given to account for coordination in the literature, providing a syntactic explanation for the properties seen in the first part of the chapter. 


\subsubsection{Types of coordination}

In coordination, the particle that is used to connect the two units is called coordinator, which can be different depending on the type of coordination. The four attested ones in English are conjunctive coordination that has and as coordinator, disjunctive coordination having or, adversative coordination which uses but, and causal coordination using for (Haspelmath 2007: 2). In this chapter, though, we will mainly focus on the first three types. We will refer to conjunctive and disjunctive coordination also as conjunction and disjunction, respectively.

(3) a. Conjunctive coordination

Snowwhite ate and drank.

b. Disjunctive coordination

She was a countess or a princess.

c. Adversative coordination

The dwarfs were ugly but kind.

d. Causal coordination

She died, for the apple was poisoned.

(Haspelmath 2007: 2)

In the examples in (3), the coordinator is a word but, in some languages, the coordinator can appear also as a prefix or a suffix attached to one of the two conjuncts, marking that the two units are combined together. Japanese, for instance, has the particle expressing and attached to the end of the first conjunct, as illustrated in (4a). In 
$\operatorname{Dargi}^{2}$ it is adversative coordination that can be expressed using a clitic element, as in (4b). Disjunction, instead, is mainly expressed using an independent element, as we saw for English above.

a. musuku-ga sotugyoo sita-si musume-ga (Japanese) son-NOM graduation did-and daughter-NOM yome-ni itta.

bride-DAT went

'The son graduated and the daughter got married.'

(Ross 1967: 90-91)

b. xan

$$
\text { yurt }
$$$$
b-i c-a
$$$$
\text { P-ili }
$$

khan(ABS) [house(ABS) N-sell-imp] say-GER

r-uqna-či $w$-äq'-I sa.y-gu r-uqna

F-old-sup M-come-GER be:M-but F-old

q'abul. $\langle r>i k-i l i$ ahen.

agree:F-GER be.not

'The khan went to the old lady and said "Sell me the house", but the old lady did not agree.'

(Haspelmath 2004: 205)

Focusing on conjunction, it is possible to express it using a different marker, that is with, realizing the so called comitative coordination, semantically equivalent to the conjunctive counterpart, as we can see in (5), as explained by Hartmann (2015: 484) for German.

\footnotetext{
${ }^{2}$ Dargi is a written language of Daghestan.
} 
a. Peter und Carlo sind nach Kassel gefahren. (German)

Peter and Carlo are to Kassel driven

'Peter and Carlo went to Kassel.'

b. Peter ist mit Carlo nach Kassel gefahren.

Peter is with Carlo to Kassel driven

'Peter went to Kassel with Carlo.'

Another element that is used to express conjunction is equivalent to the additive focus particle also that, as pointed out by Mithun (1988), can develop into a conjunctive marker. We can see an example in (6), in Lezgian ${ }^{3}$, where the particle $-n i$ means also.
(6) Isa-di-ni Ali-di sada-sada-w ğil-er
Isa-ERG-CONJ Ali-ERG one-one-ADESS hand-PL
wuga-na.
give-AOR

'Isa and Ali shook hands.'

(Haspelmath 1993: 327)

In this section we saw that there are markers to express the different types of coordination but it is common in languages to express them also using only prosody, without any overt particle, as we will see in the next section.

\footnotetext{
${ }^{3}$ Lezgian is a North Caucasian language.
} 


\subsubsection{Asyndetic and syndetic coordination}

Coordination structures usually show the presence of an overt coordinator, but they may also lack it: in that case they are realized as a juxtaposed construction in which the specific coordination relation is given by the context and by the semantics of the two conjuncts (Mauri 2008). Moreover, intonation is the only way to signal the presence of coordination (Velupillai 2012). Depending on the coordinator being overt or covert it is possible to respectively distinguish syndetic and asyndetic coordination, respectively. It is possible to refer to the latter one also as juxtaposition (Velupillai 2012). In English, the choice of using asyndetic coordination can occur in a particular context such as listing (Mauri 2008), as in (7), or choosing a stylistically marked style (Velupillai 2012). In sign languages (SLs), instead, as we will see in 2.3 for several SLs and in 3.1 for Catalan Sign Language (LSC), expressing conjunction asyndetically is the strategy most commonly adopted and prosodic cues are crucial in detecting the type of coordination.

(7) I came, I saw, I conquered.

(Mauri 2008: 60)

Rapanui ${ }^{4}$ shows juxtaposition for both phrasal and sentential coordination (8a, b), and it can also be used in disjunction (8c).

\footnotetext{
${ }^{4}$ Rapanui is an Austronesian language spoken on the Easter Island.
} 
(8)

a. he e'a kiruna, he u'i u'i a te

ACTN get up ACTN look RED POSS SPE

kona ta'ato'a.

place every

'She stood up (and) looked everywhere.'

b. he oho he tu'u he u'i, i oho era, $i$

ACTN go ACTN arrive ACT see PAST GO PPD PAST

u'I era he ki mai ki a au.

see PPD ACTN say TOW DAT P.SG 1SG

'I went off (and) came to see him, (and) when I had got

there, (and) he had seen me, he said to me...'

c. he oro ki te po'e mo kai, he inaki

ACTN grate DAT SPE po'e BEN eat ACTN accompany

koe ki te kiko.

2SG DAT SPE meat

'You grate it to make po'e (or) you use it to accompany meat.'

(Du Feu 1996: 85, 87, 88)

Mithun (1988) argues that there are several languages which do not show any overt marker and that asyndetic coordination is characterized by different intonational patterns. The two conjuncts can be separated by a pause with a non-final peak contour, but also no intonation break can be produced. Mauri (2008) reports cases of 
asyndetic coordination in Kisi, Hidi and Lango $^{5}$, among other languages.

As for syndetic coordination, looking at the number of coordinators, it is possible to use one or more, distinguishing between monosyndetic (9a) and bisyndetic coordination (9b), respectively (Haspelmath 2007: 6).
a. Franz and Susi.
b. Both Franz and Susi.

Let us focus on monosyndetic coordination. Depending on the language, the position of the coordinator can take a different position with respect to each conjunct. Its position can be ascribed to the headedness of the language, that is head initial or head final.

\subsubsection{Position of the coordinator: head initial and head final languages}

Considering only monosyndetic coordination, it is possible to identify two main types depending on the position of the coordinator: final and initial. Assuming the first conjunct is $\alpha$ and the second one $\beta$, final coordination shows a $[\alpha[\beta \&]]$ order where the coordinator appears at the end of the two conjuncts. As for initial coordination, instead, the coordinator appears between the conjuncts and it can be divided in two further types: prepositive, where the coordinator

\footnotetext{
${ }^{5}$ Kisi is spoken in West Africa, Hidi in Cameroon and Nigeria and Lango in Uganda.
} 
belongs to the second conjunct $[[\alpha][\& \beta]]$ and postpositive, where the coordinator belongs to the first conjunct $[[\alpha \&][\beta]]$.

In order to establish the distribution of the coordinator, Haspelmath (2007) proposes four tests:

i) Intonation: the coordinator forms an intonational group with one of the two conjuncts;

ii) Pauses: a natural pause can be produced either before or after the coordinator;

iii) Discontinuous order: when it is possible to separate the conjuncts with material such as temporal adverbs and the second conjunct added as an afterthought, it is possible to establish the position of the coordinator;

iv) (Morpho)phonological alternations: when the coordinator or a conjunct undergoes such alternations when establishing a relation between the two, this shows that they form a constituent together.

In languages with coordination expressed by a clitic particle, Stilo (2004) uses extraposition, trying to move the second conjunct and see if the coordinator moves along; if it does, the coordinator is considered to be part of it. Stilo (2004) considers this test more exhaustive than looking at the morphological distribution of the coordinator and therefore intonational grouping. It is possible to apply it only in languages that allow extraposition, though. Japanese, for example, is not one of those.

Applying the tests suggested by Haspelmath (2007), it is possible to affirm that English shows prepositive coordination 
$[[\alpha][\& \beta]]$, as in (10) looking at intonation and discontinuous order. In (10a, a'), // indicates the presence of an intonational break. Following Stilo (2004), also Standardized Colloquial Persian (Farsi) has prepositive coordination, despite an apparent postpositive structure and basic SOV order. As we can see in (10d), the element corresponding to and is enclitic to the preceding element but when extraposing the second conjunct, it moves along and it encliticizes onto the element that precedes it (10d'). This confirms the grouping $[[\alpha][\& \beta]]$. Japanese and Baram Kayan (Western Austronesian), instead, looking at the morphological alternations, respectively have postpositive $([[\alpha \&][\beta]])$ and final coordination $([\alpha[\beta \&]])$, in (11) and (12). Japanese, differently than Farsi, does not allow extraposition of any kind therefore it is only possible to look at the morphological properties of the coordinator.

(10) Initial, prepositive coordination: $[[\alpha][\& \beta]]$

a. The son got married // and the daughter graduated.

a'. *The son got married and // the daughter graduated

b. My uncle will come tomorrow, or my aunt.

b'. *My uncle or will come tomorrow, my aunt.

(Haspelmath 2007: 8)

d. Xoda ye dune baradar-o // ye xahardad (Farsi) God one (CLF) brother-and one sister gave beh-esh. to-3S.OBL 
d'. Xoda ye (dune) bæradær dad beh-és-o

God one (CLF) brother gave to-3S.OBL-and ye xahær.

one sister

'God gave him a brother and a sister.' (Stilo 2004: 280)

(11) Initial, postpositive coordination: $[[\alpha \&][\beta]]$

musuku-ga sotugyoo sita-si // musume-ga

(Japanese)

son-NOM graduation did-and daughter-NOM

yome-ni itta.

bride-DAT went

'The son graduated and the daughter got married.'

(Ross1967: 90-91)

(12) Final coordination: $[\alpha[\beta \&]]$

en na' uvui nah dalo' Anyi' ji Jau ji (Baram Kayan)

PRT he call FOC them Anyi' one Jau one

[Uvang ji pah].

Uvang one also

'He called them _Anyi, Jau, and Uvang.'

(Clayre and Kubit 1974: 72)

Zwart (2005) goes back to the question whether the position of the coordinator can be derived by the head-initial or head-final nature of languages. Looking at noun phrase coordination, he concludes that, in a sample of 68 head-final and 77 head-initial languages, the use of 
final coordination of the type $[[\alpha][\beta \&]]$ is very rare, while the initial coordination where the coordinator appears between the two conjuncts is widespread in both types. Data from Ross (1967) already showed that a head initial language like English forms a unit with the second conjunct, as we saw in (10a), while a head final language like Japanese shows postpositive coordination (11). Stilo (2004), though, attests prepositive coordination in Farsi, despite being a head-final language. Till now no generalization has been made with respect to the factor that can determine the presence of prepositive or postpositive initial coordination in head-final and initial languages. In chapter 3, though, we will relate this aspect to other properties of coordination in LSC and other SLs. We will also see a proposal where constituency in coordination seems to affect the characteristics of ellipsis in head-initial and head-final languages (5.6) considering a sample that includes both spoken and sign languages.

Looking now at the structure of coordination from a different point of view, it is possible to identify another relation between the conjuncts, that is symmetry and asymmetry. In the next section, we will focus on conjunction and look at the difference in meaning conveyed by these two types of relations between conjuncts.

\subsubsection{Symmetric and asymmetric coordination}

In the introduction, we anticipated the concepts of symmetry and asymmetry in coordination. These aspects have been studied from a semantic and a syntactic point of view considering both DP/NP and 
VP coordination and sentential coordination in order to determine whether the syntactic structure for coordination needs to be flat or hierarchical. In this section, we will focus on the semantic relation between conjuncts while we will look at the syntactic properties in 2.2 .

Semantically, it is possible to establish a symmetry, a parallelism, between the conjuncts in several ways. Crysmann (2006) affirms that in order to have a semantic parallel between two DP conjuncts, they need to realize the same theta role; when having two VPs, they need to have the same "degree of semantic saturation", that is the verb in each conjunct needs to be fully saturated, whether the verb is transitive or intransitive. Hartmann (2015: 491) provides examples for the first case. In (13) we can see that in German it is not possible to realize coordination of DPs that have different theta roles where the first conjunct is an agent and the second one a recipient. In (14), Crysmann (2006: 184) shows how in English both verbs need to be semantically saturated.

(13) Das Kostüm hat [DP Gerda] (*und) [DP ihrem (German) The costume has Gerda and her Enkelkind] geschenkt. grandchild given 'As for the costume, Gerda gave it to her grandchild.'

(14) a. *We will attack the enemy and beat.

b. We will attack and beat the enemy. 
Another factor that makes two conjuncts symmetric or asymmetric is the temporal relation between them. Nonato (2014), citing Ross (1967) and Postal (1998), defines coordination as symmetric when "conjuncts can be swapped without affecting the semantics of the coordinate complex", as in (15). As for the asymmetric one, instead, it is found when "swapping conjuncts results in a different meaning", as in (16).

(15) Symmetric Coordination (SC)

a. Matthew dates a veterinarian and hopes to date a surgeon.

b. = Matthew hopes to date a surgeon and dates a veterinarian.

(16) Asymmetric Coordination (AC)

a. You can use this magic herb and get cured of cancer.

b. $\neq$ You can get cured of cancer and use this magic herb.

Nonato (2014: 5)

Moreover, as pointed out by Blakemore \& Carston (1999), symmetric coordination, differently from the asymmetric one, does not require a temporal or causal relation between two clauses. Mauri (2008) explains that it is possible to distinguish between two types of temporal relation: sequential and simultaneous. In the first case, we will have an asymmetry between the conjuncts, differently from when the two events expressed are happening at the same time. In order to recognize the sequential type, the conjuncts cannot be reversed, as in (17a), otherwise the meaning of the whole sentence would be different (17b). In languages like Malagasy, instead, a 
sequential coordinator, dia, meaning "and then", can be used for this purpose (17c).

(17) a. The police came into the room and everyone swallowed their cigarettes.

b. Everyone swallowed their cigarettes and the police came into the room.

(Lakoff 1971: 127)

c. Nitsangatsangana aho dia (Malagasy)

PAST.AT.RED.walk 1SG.NOM DIA

hitako ny bokibo very.

found.1SG DET book.1SG lost

'I was walking in the woods, and then I found my lost book.'

Simultaneous combination, instead, is characterized by absence of sequential order since the two conjuncts are happening at the same time (18), and thus creates a symmetry between them.

(18) At noon, I was eating at the park and Mary was sitting next to me.

(Mauri 2008: 84)

It is also possible to identify an atemporal relation, that is the temporal relation between the conjuncts is not relevant. Looking at 
(19) from Mauri (2008), it is clear that the order between the two events is not crucial.

(19) When we came back home, I took a shower and Paul checked his e-mail.

(Mauri 2008: 85)

It is interesting to notice, though, that the two events might have happened at the same time. The expression of atemporal and simultaneous combination is mostly expressed using the same type of structure since in both types of combination the order of the conjuncts is absent or not relevant (Mauri 2008). The two conjuncts do not need to be presented in a special order, having in both cases a symmetric relation.

As pointed out by Mauri (2008), when having different participants in an atemporal combination, though, it is possible to create a contrast between the two conjuncts, as in (20). In (20), John and Mary and the respective VPs are contrasting.

(20) John works in a store and Mary is still a student.

(Mauri 2008: 86)

As also noticed by Repp (2010), contrast is another relation that results in a symmetric coordination structure. Hartmann (2015: 493) defines two expressions to be in contrast when "they belong to the same semantic field without being identical". We can see an example in (21), where the two DPs are contrasted. Repp (2016: 1), moreover, 
with respect to two sentences, states that "S1 and S2 may be construed as being in a contrastive relation if $S 1$ contains an element $\alpha$ that can be construed as an alternative to an element $\beta$ in S2, where being construed as an alternative reflects the notions of juxtaposition and comparison". Umbach (2004: 2), as well, talks about set of alternatives where "picking one element out of a set of comparable entities is often regarded as establishing a contrast between the one element and the others". In (22) we can see an example from Umbach (2004) where the words in capital letters are intonationally marked and contrasting with each other between conjuncts. Moreover, the conjuncts can be put in a sequential or a contrastive discourse relation using the coordinator and or but, respectively.

(21) Die Lehrer und die Busfahrer sind in den (German) the teacher and the bus.drivers are in the Streik getreten.

strike stepped

'The teacher and the bus drivers have gone on strike.'

(Hartmann 2015: 493) /JOHN went to IPARIS and/but /MARY went to LONDON. (Umbach 2004: 2)

Moreover, there are three main types of contrast that are found in adversative coordination, as we will see in detail in 2.2.5.2: oppositive, corrective and counterexpectative. 
As for oppositive contrast, in (20) repeated in (23a), the fact that the two conjuncts are parallel but different creates opposition. Lakoff (1971) would define the conjuncts to be in semantic opposition. Correction, instead, is expressed through the negation of the first conjunct and its substitution in the second one, as in (23b), from Mauri (2008). Counterexpectative contrast, finally, is generated by the negation of an expectation which can be explicit or provided by the context. An example from Lakoff (1971) is given in (23c).

(23) a. John works in a store and Mary is still a student.

(Mauri 2008: 86)

b. Peter is not studying in his room, but he's playing in the garden.

(Mauri 2008: 130)

c. John is tall but he's not good at basketball.

(Lakoff 1971: 133)

In (23c), asserting that John is tall gives the expectation that he is also good at playing basketball. Such assumption is denied, though, in the second conjunct.

These types of relations are mainly expressed through the use of an overt marker. Referring to the language sample used by Mauri (2008), Lango, as well as other languages such as Turkish and Georgian, uses juxtaposition. These languages can also have an overt marker to express counterexpectative contrast, as in Lango and Limbu, or oppositive or corrective contrast as in Turkish and Georgian. At the spoken level, it is possible to find juxtaposition also 
in languages like English, as in (24), where intonation helps in identifying correction.

(24) Peter is not studying in his room, he is playing in the garden. (Mauri 2008: 130)

It is important to notice, though, that in all these examples of symmetric coordination based on temporal order or contrast, symmetry is not necessarily respected if an element like a possessive pronoun is used in the second conjunct. An example like the one in (25a), repeated from (18), in which the symmetry is given by the atemporal relation between the conjuncts, needs to be considered asymmetric if adding an element like a possessive adjective that requires an antecedent. In (25a), the antecedent of her is the subject of the first conjunct and this relation makes impossible to reverse the two conjuncts. The same would happen with the use of a quantifier.

(25) a. At noon, I was eating at the park and Mary was sitting next to me.

b. At noon, Mary was eating at the park and her daughter was sitting next to her.

Therefore, despite the superficial semantic relation between the conjuncts, it is necessary to take into account the potential structural relation between them. As we will see in 2.2, there are other syntactic arguments that lead to the same conclusion. 
Another relation that is possible to find in asymmetric coordination, expressed syndetically or asyndetically, is dependency between the conjuncts. It is often called semantic subordination. $\mathrm{Na}$ \& Huck (1992) and Schmerling (1975) use the term asymmetric coordination instead of pseudo-coordination, which states the relation of coordination with subordination (de Vos 2005, among others), to underline the fact that one conjunct is semantically more prominent than the other. We can see two examples in (26), (26a) from Culicover and Jackendoff (1997) and (26b) from Hoeksema \& Napoli (1993), respectively.

(26) a. One more can of beer and I'm leaving.

b. I fainted, the sun was so hot.

(26a) expresses a conditional relation between the conjuncts, while (26b) expresses a causal one. In (26b), prosody is crucial in conveying a subordinate interpretation. As claimed by Hoeksema and Napoli (1993), the two clauses in (26b) are produced under a unique intonational contour. Prosody plays an important role in distinguishing between semantic subordination and coordination when dealing with juxtaposition. This is the case for languages like Otomi ${ }^{6}$ where the presence of a prosodic break between the conjuncts deletes the subordination reading that is present when there is a single intonational contour over the two sentences.

A recent classification of the different types of asymmetric coordination appears in Weisser (2015), who distinguishes between:

\footnotetext{
${ }^{6}$ Otomi is a Mesoamerican language family of the Oto-Manguean stock.
} 
conative, contiguous, scene-setting, consecutive and conditional coordination. All the cases of asymmetric coordination described by Weisser (2015) show properties that associate these structures semantically and structurally with coordination and subordination. Some structures show both syntactic and semantic properties in common with subordination, but those will not be described in depth. For a complete description and analysis, I refer the reader to Weisser (2015).

As for conative coordination, in (27), it is characterized by having always the verb try in the first conjunct and the second one being, in a certain sense, the infinitival complement of try.

(27) He wanted to try and finish this thesis.

(Zwart 2005: 1)

In contiguous coordination, in (28), the activity described by the first conjunct cannot be considered different from the one of the second one. This relation makes the second conjunct aspectually dependent on the first one (de Vos 2005). The class of verbs that can occur in the first conjunct is very restricted (go, come, sit) and in this kind of structure they are considered semantically bleached since they almost lose their meaning. As pointed out by Weisser (2015), the verb in first conjunct in (28) does not really denote the action of going somewhere.

(28) He went and addressed the troops. 
Ross (2013: 10) introduces these two types of asymmetric coordination as try-type and go-type. Culicover and Jackendoff (1997) refer to them as showing "semantic subordination despite syntactic coordination". Often, it is possible to have an infinitival paraphrase (29).

(29) a. I try and do it. = I try to do it.

b. I go and get it. $\approx$ I go to get it.

A diagnostic to distinguish the two types, proposed by Ross (2013), is given by the fact that in the try-type it is often possible to cancel the second verb's truth conditions, like in (30), while that is not allowed in the go-type .

(30) a. I will try and finish the report on time, but I might not succeed.

b. *I will go and get the book, even if it is sold out.

(Ross 2013: 12)

As for scene-setting coordination, in (31), the verb in the first conjunct seems to set the temporal or locative information for the second conjunct. Differently from contiguous coordination, it is possible to insert a PP or a particle between the conjuncts. Moreover, the set of verbs that can be used in this structure is not so limited.

(31) He went (to the store) and bought the whiskey.

(adapted from Ross 1967) 
De Vos (2005) focuses on distinguishing the last two types of asymmetric coordination since in the literature they have been largely considered to be the same ${ }^{7}$.

In consecutive coordination, in (32), the second conjunct appears as a consequence of the first one, differently than in scenesetting coordination. These two types of coordination are considered very similar apart from their semantics because, in the latter one, it is the first conjunct that gives temporal or background information.

(32) The guys in the Caucasus drink that stuff and live to be a hundred.

(Lakoff 1986: 157)

The last type of asymmetric coordination studied by Weisser (2015) is conditional coordination. An example like (33) expresses a conditional meaning where the first conjunct denotes a condition for the event in the second one to occur.

(33) You drink another can of beer and I'm leaving.

(Culicover \& Jackendoff 1997: 197)

Differently from the previous ones, conditional coordination is the only one where it is possible to have different subjects in both conjuncts.

${ }^{7}$ De Vos (2005) proposed a battery of tests that involve violation of syntactic properties of coordination, insertion properties, subject licensing, semantic bleaching and VP deletion. 
On the base of these data it was possible to look at the relation between coordination and subordination, both in asyndetic and syndetic coordination.

In this section, we looked at the description of coordination structurally and semantically. In the next one, section 2.2 , we will focus on the syntax of coordination.

\title{
2.2. Syntax of coordination
}

The main questions that have been asked in relation to the syntax of coordination are the following:

1. Is there asymmetry between conjuncts?

2. What categories can be coordinated?

3. What are the properties of extraction in coordination?

4. What is the structure that better represents coordination in syntax?

\begin{abstract}
All these questions are related to the need of justifying whether coordination must be analyzed as a flat structure, as in (34a), or a hierarchical one, as in (34b), and whether coordination actually constitutes a special syntactic category or it depends on the type of units that are coordinated.
\end{abstract}

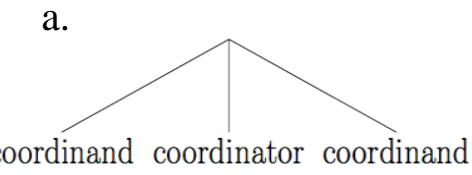

b.

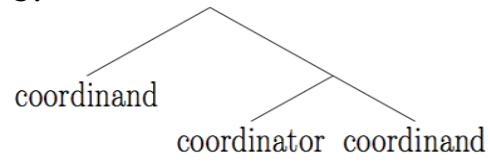


In the following sections we will try to answer the questions presented above concluding with the presentation of the main proposals made to analyze coordination syntactically.

\subsubsection{Asymmetries in coordination}

The answer generally given to the first question introduced above ( $I s$ there asymmetry between conjuncts?) is "yes". In section 2.1 .4 we already mentioned cases of asymmetric coordination considering asymmetry in the categories of the conjuncts that it is possible to coordinate and also in the semantic relation between them. Other factors that justify asymmetry in coordination are:

i) the position of the coordinator with respect to the conjuncts

ii) binding

iii) possessee pronominalization

iv) unbalanced coordination.

We will now examine each one in turn.

(i) As mentioned in section 2.1.3, on the basis of tests such as intonation, pauses, discontinuous order and (morpho)phonological alternations (Haspelmath 2007), it is possible to see that the coordinator can assume different positions with respect to the conjuncts. We can see another example from English in (35) from Ross (1967) who noticed that in English the coordinator forms a unit with the second conjunct. 

a. John left, and he didn't even say good-bye.
b. John left. And he didn't even say good-bye.
c. *John left and. He didn't even say good-bye.

(Ross 1967: 90-91)

Data like that in (35) and in the previous section 2.1.3 show that the two conjuncts are asymmetric cross-linguistically, even in cases when the coordinator is a clitic.

(ii) A second argument that proves the asymmetry between conjuncts is binding. Focusing on conjunction in English, the first aspect to consider is that the first conjunct can be the antecedent of a pronoun in the second one but the second conjunct cannot be the antecedent of a pronoun in the first conjunct (36).

(36) a. Every mani and hisi dog went to mow a meadow.

b. *Hisi dog and every mani went to mow a meadow.

(Munn 1993: 16)

Several authors, though, point out that examples like the ones in (36) do not tell us much about the binding relation between conjuncts. The quantifier every c-commands his only at the Logical Form (LF) via quantifier raising (QR), as mentioned by Progovac (1998). It is the quantifier in an A-bar position to c-command the pronoun and not its trace. This is also the case of the relation between quantifiers and epithets (37), where the latter can be a bound variable. Also the epithet in (37) is c-commanded at LF. 
(37) John criticized every senator in private while praising the bastard $_{\mathrm{i}}$ in public.

Moreover, at the sentential coordination level, variable binding cannot be invoked either, since c-command operates only within sentence boundaries (Progovac, 1998), as in (38).

*Nobody $y_{i}$ joined the army and his mother joined the navy. ${ }^{8}$

(Carminati 2002: 4)

Cases of binding with quantifiers, then, do not give any evidence for binding between conjuncts. Progovac (1998: 208) gives another argument against c-command in coordination, looking at Negative Polarity Items (NPIs). As we can see in (39), the NPI in the first conjunct does not c-command the one in the second one.

*He chased nobody and/or any dogs.

The same holds also for Spanish, as reported by Camacho (2003), but the reason of the ungrammaticality of (40a) and therefore also of (39) seems to be the lack of collective reading that is instead present in the grammatical (40b). (40b) means that nobody saw pairs formed by Juan and each woman.

\footnotetext{
${ }^{8}$ Authors such as Oehrle (1987), McCawley (1993) and Johnson (2009) claim that in cases of gapping, though, there is c-command of the first conjunct over the second one when having a quantifier such as no (e.g., No $o_{i}$ woman can join the army

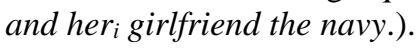


a. *Nadie vio a Juan y ninguna mujer. nobody saw to Juan and no woman

'Nobody saw Juan and any woman.'

b. Nadie vio a ninguna mujer y Juan. nobody saw to no woman and Juan 'Nobody saw any woman and Juan.'

(Camacho 2003: 21)

The collective reading seems, then, to be a factor that needs to be present in order to have c-command between NPIs in coordination. Looking at the elliptical structure of gapping in Spanish in (41), though, an NPI in the first conjunct can c-command an NPI in the second one, without involving collectivity. The NPI under discussion here is the Spanish postverbal tampoco that is similar to the English “(n)either". The NPI nadie 'none/anyone' is licensing tampoco in the second conjunct via c-command. As confirmed by the examples in (41b) and (41c), absence of negation in the first conjunct and the eventual presence of it in the second but not in the first one, does not license the NPI tampoco.

(41) a. Nadie vino y Miguel tampoco. no-one came and Miguel neither 'No one came, and neither did Miguel.'

b. *Juan vino y Miguel tampoco. Juan came and Miguel neither

c. *Juan vino y Miguel no tampoco. Juan came and Miguel not neither

(Camacho 2003: 21) 
The data in (40) and (41) show that c-command in coordination is indeed present. Moreover, Zhang (2010), citing Sag et al. (1985: 117), underlines the fact that in (42) the pronoun it pronominalizes the entire first conjunct a Republican and is co-referential with it.

(42) Pat is a Republican and proud of it.

Therefore, since it is not possible to invert the two conjuncts, there must be an asymmetry. It is possible to observe the same in (43) where there is clausal coordination but it is impossible to invert the two conjuncts a cause of the presence of a possessive pronoun in the second one. There is no real binding relation but there is still asymmetry between the two conjuncts.

(43) Mary jought pizza and her ${ }_{j}$ mother brought ice-cream.

It is possible to conclude, then, that the first conjunct is in a higher position than the second one.

iii) A third argument to show the asymmetry between conjuncts is related to possessee pronominalization. In (44), the impossibility of inverting the order of the conjuncts shows that the first conjunct is structurally higher than the second one.

(44) a. Sally's mother and yours have turned vegetarian.

b. *Yours and Sally's mother have turned vegetarian.

(Zhang 2010: 12) 
iv) The last argument is built on unbalanced coordination (Johannessen 1998) where case is involved. Unbalanced coordination is characterized by unexpected case assignment where accusative can be assigned to subjects and nominative to objects. Interestingly, in unbalanced coordination, only the second conjunct is affected (45).

(45) a. She and him will drive to the movies.

(Schwartz 1985: 165, quoted in Johannessen 1998)

b. He says he saw John and I last night.

(Quirk et al. 1985: 338, quoted in Johannessen 1998)

Johannessen (1998) provides data from 32 languages showing that the phenomenon is systematic and it is attested cross-linguistically. Again, this is another argument to prove asymmetry between conjuncts.

The properties we saw in this section have an effect in the directionality of the syntactic structure of coordination. Without taking any part on the category of coordination, in the next section we will see how the distribution of the conjuncts in relation to the coordinator is affected.

\subsubsection{Directionality of coordination}

The location of the deviant conjunct in unbalanced coordination and the position of the coordinator with respect to the conjuncts can be used as arguments to define the directionality of coordination 
depending on the word order, as stated by Johannessen (1998), who establishes the correlation reported in (46).

\section{(46) Johannessen's Correlation}

"There is a very strong correlation between, on the one hand, the order of verb+object, and on the other, that of normal conjunct+deviant conjunct (usually the same as that between conjunction+deviant conjunct)."

In other words, depending on the word order of the language, it is possible to predict the position of the deviant conjunct, the one affected by unexpected case. In SVO languages, it will be in second position (47a), and in SOV languages, in first position (47b).

(47)

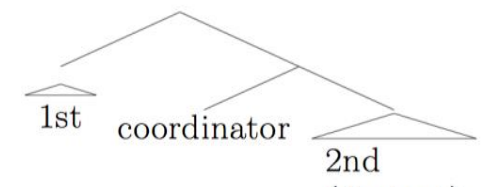

(deviant) b. SOV languages

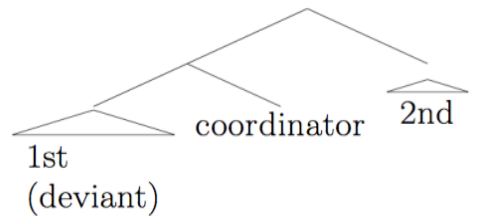

Another argument that affects directionality of coordination is the position of the coordinator. As seen in the previous section 2.1.3, the coordinator can take a specific position with respect to the conjuncts when looking, for example, at the prosody of a sentence. When appearing only once, or more than once as in doubling coordination, the coordinator can be to the left of the second conjunct, realizing prepositive coordination, or to the right of the first one, having postpositive coordination: $[[\alpha[\& \beta]]$ or $[[\alpha \&][\beta]]$. The former is 
found, for instance, in English (48a) and the latter in Japanese (48b), from Ross (1967: 90-91). An example of doubling coordination in Italian (Progovac 1998: 4) and Japanese (Kayne 1994 and Zoerner 1995) are shown in (49). The coordinator is found to the left in Italian, and to the right in Japanese, respectively.

(48) a. The son graduated // and the daughter got married.

b. musuku-ga sotugyoo sita-si //musume-ga (Japanese) son-NOM graduation did-and daughter-NOM yome-ni itta.

bride-DAT went

'The son graduated and the daughter got married.'

(49) a. Sono arrivati (e) Anna, (e) Roberto, e Laura. (Italian) are arrived and Anna and Roberto and Laura. 'Anna (and) Roberto and Laura have arrived.'

b. [Robin-to Kim-to Terry-to]-ga (Japanese) Robin-and Kim-and Terry-and-CASE

Zhang (2010) argues that the two orders need two different kinds of coordination structure: right-branching for prepositive coordination, like in English (50a), and left-branching for postpositive coordination, like in Japanese (50b).

a.

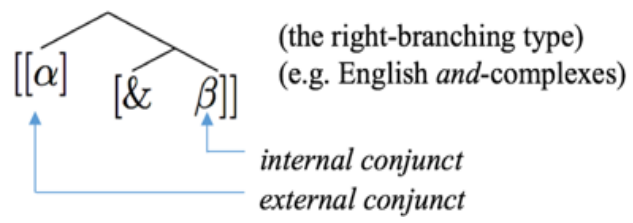


b.

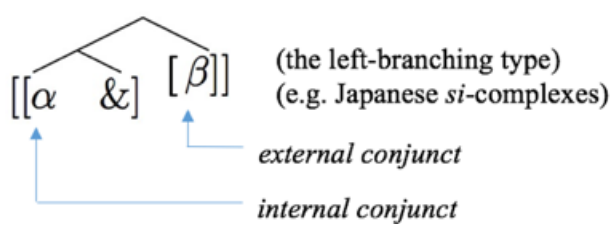

In section 2.1.3, though, we saw that Standardized Colloquial Persian (Farsi), despite having the coordinator morphologically attached to the first conjunct, it appears grouped with the second conjunct: $[[\alpha[\& \beta]]$ (Stilo 2004). With regard to what was pointed out by Zhang (2010) in (50), also Farsi shows a right-branching coordination structure for coordination based on the data presented in 2.1.3. In 2.2.4 we will see that the position of the coordinator could be an argument to consider coordinators heads since a head is expected to behave as such also with respect to the word order of a language: in VO languages the head is expected to appear before the complement and in OV languages it is the complement that precedes its head (Svenonius 1992). This generalization also relates to Johannessen's Correlation in (46). As we saw in 2.1.3, Farsi, and also Catalan Sign Language (see 3.2.2), do not respect this generalization, though. Farsi, despite being a head final language, shows a right-branching coordination structure established by the position of the coordinator. Further data on the directionality of coordination will be presented in chapter 4 when talking about gapping. The grouping between conjunct and coordinator seems to determine not just the directionality of the coordination structure but also the properties of ellipsis in a language. As for the arguments to consider coordinators heads, we will see more solid ones in 2.2.4. 
Let us go into the second question that was addressed at the beginning of this main section 2.2.

\subsubsection{What can be coordinated?}

An important issue that has been central to coordination is related to what syntactic and semantic categories can be coordinated.

Chomsky (1957), using examples like the ones in (51), points out that different syntactic categories cannot be coordinated. He formulates the so-called Coordination of likes constraint (CLC) which states that "if $\mathrm{X}$ and $\mathrm{Y}$ are both constituents, but are constituents of different kinds...then we cannot in principle form a new sentence by conjunction". Williams (1978), following the same line, formulated the Law of coordination of likes ( $L C L)$.

(51) a. The scene [PP of the movie] and [PP of the plan].

b. *The scene [pP of the movie] and [cP that I wrote].

Schachter (1977) then specified that constituents "need to belong to the same syntactic category and have the same semantic functions".

However, the examples in (52) show that it is perfectly possible to coordinate syntactically unlike categories.

(52) a. Pat has become a banker and very conservative.

(Sag et al. 1985: 118)

b. Robin is ugly, a dolt and of no help. 
c. Robin considers Kim completely evil, a total witch, and beyond salvation.

d. [NP Robin's help] and [CP that (s)he gave it so willingly] delighted Kim.

e. Robin realized [CP that the sky was falling] and [NP the gravity of the situation].

(Zoerner 1995)

Focusing on (52d) and (52e), it is possible to notice that NPs and CPs can be coordinated as subjects or objects of a verb. Examples like the ones in (53), though, show a limitation in the selection of the first conjunct where the main verb is a phrasal verb.

(53) a. You can depend on [my assistant] and [that he will be on time].

b. *You can depend on that he will be on time.

c. *You can depend on that he will be on time and my assistant.

(Sag et al. 1985: 165)

In (53), the first conjunct cannot be clausal but the second one can be if the first one is a conjunct that satisfies the category selection of the verb. It is only the first conjunct, then, that "must satisfy the category requirements that are imposed on the whole coordinate complex", as underlined by Zhang (2010: 54).

The discussion related to what categories are possible to coordinate is also very important in determining the adequate 
representation of coordination and eventually the category for the phrase to select for it.

In order to give the proper representation to the syntactic structure of coordination, it is also crucial to look at the extraction properties of coordination, topic of the third question asked at the beginning of section 2.2, that again will put in contrast symmetric and asymmetric coordination.

\subsubsection{Syntactic properties: CSC and ATB}

Two important properties in relation to extraction in coordination that correlate to each other are Coordinate Structure Constraint (CSC) and Across-the-board (ATB) movement.

Ross (1967) states for CSC that "in a coordination structure, no conjunct may be moved, nor may any element contained in a conjunct be moved out of that conjunct". (54a) gives an example of the first part of this definition, showing the unacceptable movement of a whole conjunct to an A-bar position. (54b), in turn, illustrates the second part, the unacceptability of extracting out of a conjunct.
a. $*$ Which surgeon ${ }_{i}$ did Kim date $t_{i}$ and a lawyer?
b. *Which surgeon ${ }_{\mathrm{i}}$ did Kim date friends of $\mathrm{t}_{\mathrm{i}}$ and a lawyer.

(Progovac 1998: 5)

These two parts illustrated in (54) are named by Grosu (1972, 1973) the Conjunct Constraint (CC) and the Element Constraint (EC), respectively. The violation of the CSC is restricted to symmetric 
coordination while it does not apply to asymmetric coordination, where extraction of a whole conjunct or of a part of it is allowed, as in (55): (55a) is a case of extraction in scene-setting coordination and (55b', b') in continuous coordination, both mentioned in section 2.1.4. This difference between symmetric and asymmetric coordination in respect to extraction is also an argument used by Zhang (2010) in order to support her proposal where CSC cannot be a general syntactic constraint for movement.

(55) a. How much $_{i}$ can you drink $t_{i}$ and still stay sober?

(Lakoff 1986: 152)

b. John went and read a book on the bus.

b'. What did John go and read on the bus?

b'. Who went and read a book on the bus?

(Ross 1967)

$\mathrm{CSC}$, though, can also be violated in symmetric coordination if the extraction takes place out of both conjuncts, having then Across-theBoard (ATB) movement, where it is necessary to "move a constituent out of all the conjuncts of a coordinate structure" (Ross 1967). In other words, the only extracted element moves out of both conjuncts simultaneously. This is exemplified in (56).

(56) a. Which book does [Peter like _ ] and [Susan hate _ ] ?

b. *Which book does [Peter like _ ] and [Susan hate Moby Dick]? 
c. *Which book does [Peter like Moby Dick] and [Susan hate $\_$] ?

An assumption related to ATB is that the element moved out of each conjunct is subject to an identity requirement, that is, the extracted element needs to be the same for both conjuncts. Munn (1992), though, notices that some cases allow a pair-answer reading, like in (57).

(57) a. A: Where did Mary vacation and Bill decide to live?

B: Mary vacationed in Paris and Bill decided to live in Toronto

b. A: How tired did Bill look and Mary seem?

B: Bill looked exhausted and Mary looked OK.

c. A: Why did Bill leave and Fred arrive?

B: Bill left because Fred arrived and Fred arrived because he had a meeting.

An explanation has been given, semantically, by Chierchia (1993) and, syntactically, by Citko (2007), among others. Chierchia (1993), particularly looking at pair list reading of wh/quantifier questions, argues, as reported by Munn (1999: 243), that "pair-list readings of wh/quantifier questions are derived via the functional reading of the wh-element". In (58b) is given the logical form that corresponds to a pair-list or to an individual answer. 
a. What did everyone bring to the party?

b. For which $f$ : everyone $\mathrm{x}$ [x brought $f(\mathrm{x})$ to the party]

According to Chierchia (1993) and Munn (1999), ATB is not violated since under the functional reading the extracted element is the same because the function is the same.

Citko (2007), adopting the treatment of movement on the base of Copy, Merge and Delete (Chomsky 1995), gives an explanation that accounts for both answers in $(59 \mathrm{~b}, \mathrm{c})$ that reduces the analysis to which copies are deleted at LF.
a. How many books $s_{i}$ did John like $\mathrm{t}_{\mathrm{i}}$ and Mary dislike $\mathrm{t}_{\mathrm{i}}$ ?
b. Seven.
c. John liked 5 books and Mary disliked 8 books.

(Citko 2007: 1)

Citko (2007) assumes that both lower copies of the wh-phrase are deleted at PF for both interpretations, single individual (60b) and pair-list reading (60c). At LF, the single-individual reading involves the deletion of the lowers copies (60b), while the pair-list reading needs the deletion of the upper copy (60c).

(60) a. Copy and Merge how many books in [Spec,CP]:

[ст How many books did [\&P [тP John like how many books] and [тР Mary dislike how many books? ] ] 
b. Delete lower copies at LF (single individual reading):

[СР How many books did [\&P [тР John like-how many books] and [тр Mary dislike how many books? ] ] ]

c. Delete upper copy at LF (pair list reading): [сР How many books did [\&P [тP John like how many books] and [тр Mary dislike how many books? ] ] ]

(adapted from Citko 2007)

Despite the realization of non-identity readings in ATB extraction, the crucial aspect is the possibility of violating the CSC also in symmetric coordination. Extraction in both symmetric and asymmetric coordination is an important aspect to consider for the syntactic structure that will represent coordination.

Let us look at the main syntactic proposals that have been made to account for the properties we saw till now, answering to the last question of this section 2.2: what is the structure that better represents coordination in syntax?

\subsubsection{Towards a syntactic structure}

Since the ' $80 \mathrm{~s}$, linguists tried to provide an appropriate structure for coordination ranging from flat structures, to non-flat ones and to multidimensional ones. Nowadays the debate is still open even though the proposed approaches, that will be presented below, contributed to enrich and clarify our knowledge on coordination. 


\subsubsection{Flat structures}

The first attempt to propose an appropriate structure for coordination is made by Jackendoff (1977), Chomsky (1981), and many others after them. They suggest a multi-headed flat structure of conjuncts that belong to the same category, like the one in (61) that can be used for any category, for example NP as in (61b).

(61)

a.

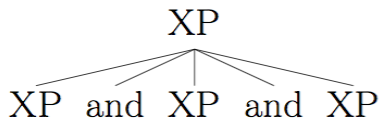

b.

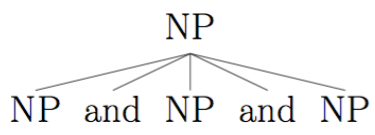

This structure, though, leads to some problems. Progovac (1998) points out that the representation in (61) cannot capture asymmetry and does not allow to coordinate different categories. Moreover, the order of conjuncts is not always reversible and this structure would not be able to account for cases like the contrast in (62), where the verb can select as first conjunct only an NP, while the second one can belong to another category.

(62) a. You can depend on [NP my assistant] and [CP that he will be on time].

b. *You can depend on [CP that he will be on time] and [NP my assistant].

The possibility of coordinating any conjunct of the same category in a flat structure also goes against the Clausal Conjunct Hypothesis 
(CCH) proposed by Gleitman (1965) and Tai (1969). The CCH states that all conjuncts are clausal and that apparent non-clausal conjuncts are the result of reduction. This operation is considered also by other authors who do not support a flat structure for coordination. As pointed out by Zhang (2010), CCH is characterized by two main operations: deletion (63) and regrouping. The former derives phrasal coordination constructions (Transformation of coordinationreduction). The latter, instead, contributes to adjusting verb agreement. In example (64), the verb passes from being singular (64b) to being plural (64a).

(63) a. Louise and George rode bicycles.

b. Louise rode bicycles and George rode bicycles.

(64) a. Tom and Jane eat bread and crackers respectively.

b. Tom eats bread and Jane eats crackers.

(Tai 1969: 144)

Important arguments against $\mathrm{CCH}$ are given by Lakoff and Peters (1966), who refuse reduction since it would give a different and wrong reading for sentences such as the one in (65).

(65) John and Mary are alike.

$\neq *$ John is alike and Mary is alike.

Zhang (2010) gives some arguments against CCH as well:

i) There are certain sentences where assuming clausal coordination reduction would lead to semantically anomalous sentences where the 
subject could not be the same in the two allegedly underlying sentences (66).

(66) a. The children are in the bathroom and in the living room.

b. \#The children $n_{i}$ are in the bathroom and the children ${ }_{i}$ are in the living room.

ii) Looking at asymmetric coordination, which is assumed to encode a single event, it would be impossible to derive this aspect from the reduction of two clauses (67), as reported by Sag. et al (1985: 151) citing Schmerling (1975: 220-221).

a. I went to the store and bought some whiskey. (1 event)

b. I went to the store and I bought some whiskey. (2 events)

Flat structures, then, are not appropriate to account for asymmetric coordination but neither for cases of symmetric one. Nonato (2014) argues for a flat structure to account for symmetric coordination, but as pointed out by Weisser (2015), no argument such as binding or conjunct agreement are taken into consideration in Nonato's work. Flat structures, then, can be excluded also in cases of symmetric coordination.

Let us go now through the main proposals that have been made for a non-flat structure for coordination. 


\subsubsection{Non-flat structures}

Assuming the necessity for a structure that shows the asymmetry between conjuncts, different structures have been proposed. They all assume coordinators to be heads based on the head-criteria of word order, uniqueness and $X^{\circ}$ element by Svenonius (1992). In section 2.2.1.1 we already looked at the criterion that relates the behavior of a head to the word order of a language. As already explained, though, SOV languages such as Farsi do not respect this criterion because, contrary to expectation, the head precedes the complement instead of following it. As for uniqueness, Svenonius (1992) claims that it is the property of a head to be unique and not reiterated inside one conjunct. As we saw for English till now, only one coordinator is allowed per conjunct. In cases where it is repeated at the begging and the end of a constituent, the initial one will be rather considered an adverb than a head. The $X^{\circ}$ element criterion, finally, shows that in coordination, the coordinator can only be a head, while the conjuncts can be either heads or maximal projections.

Among the non-flat structures that consider the coordinator a head, the main differences are based on the category used to indicate the phrase headed by the coordinator and on the status of the conjuncts, whether adjuncts or specifiers. The different structures are characterized by the following properties:

a) Conjuncts are specifiers and complements of \&P (Munn 1987; Zoerner 1995; Johannessen 1998; Weisser 2015 for asymmetric coordination); 
b) Each conjunct is the complement of its own \&P (Collins 1988);

c) Conjuncts are adjuncts and coordinators are heads of \&P (Munn 1993);

d) Conjuncts are heads but not of \&P (Camacho 1997; Zhang 2010): the category of coordination phrase depends on the category of the first conjunct.

Moreover, another type of approach is given by Goodall (1987), among others adopting his proposal (Muadz 1991 and Moltmann 1992), who proposes a multidimensional analysis where the coordinated structure is characterized by parallel levels. Coordination is seen as a union of phrase markers merged together at an identical node.

In the next subsections we will go through each proposal in detail.

\subsection{Conjuncts are specifiers and complements of $\& \mathrm{P}$}

The first analysis we are going to look at proposes that in the coordination structure the conjuncts are specifiers and complements of \&P. The main supporters of this analysis are Zoerner (1995) and Johanessen (1998), based on previous analysis such as Munn (1987). The three approaches are slightly different but they all share the idea that the conjunct is the head of a phrase, coordination phrase. The coordination head, then, has the same properties of other heads, since coordinators are considered functional categories. As for the category 
of coordination phrase, Munn (1987) calls it BP (Bolean Phrase), Zoerner (1995) \&P and Johannessen (1998) CoP, but it is just a matter of labelling. From now $\mathrm{I}$ will be using $\& \mathrm{P}$ to refer to coordination phrase in order to avoid confusion with the labelling.

Munn (1987) noticed the necessity of having a hierarchical and asymmetrical structure for coordination on the basis of examples like the one in (68) where we can see that the order of the two conjuncts cannot be inverted. This tells us that there is a hierarchical relation between the two where the pronoun his needs a referent previously introduced. Moreover, Johannessen (1998) assumes that coordinators can head an independent projection because they form a closed class and they are unstressed elements.

(68) a. John $n_{i}$ and hisis son went to the swimming-pool.

b. *Hisi son and Johni went to the swimming-pool.

The structure proposed by the three authors is the one in (69).

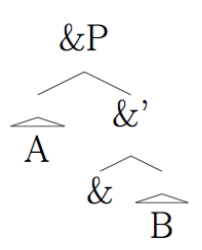

When dealing with more than two conjuncts, Johannessen (1998) assumes that for each conjunct there is a different \&P (CoP) projected, like in (70a), where the second $\& \mathrm{P}$ is marked with the 
subindex 2. Zoerner (1995), instead, assumes that there is a single \&P structure that projects more than one level, like in (70b).

(70)

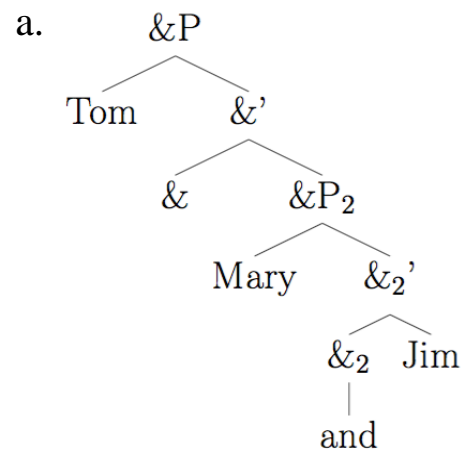

b.

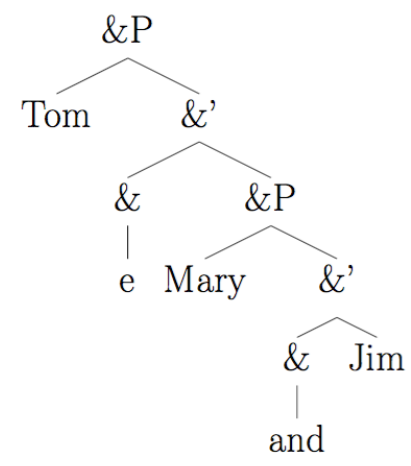

As pointed out in Progovac's (1998) overview, Zoerner's and Johannessen's approaches can differentiate sub-group coordination, cases like the one in (71). In the example, the second and the last conjunct form a sub-group. Both structures in (70) account for the asymmetry between the first and the other two conjuncts thanks to the use of a second \&P. The structure in (70a) by Johannessen, though, would be more appropriate since it would be able to account for different coordinators having two distinct \&Ps, like in (72). In Zoerner's (70b), instead, the coordinator, if overtly pronounced in both $\&$ P heads, needs to be the same.

(71) Tom and [Mary and Jim].

(72) a. Tom 'n [Mary and Jim]

b. Tom and [Mary 'n Jim]

(Progovac 1998: 3) 
For Borsley (2005), though, none of the two can account for a collective or distributive interpretation of a sentence with more than two conjuncts, like the one in (73), where either each participant takes part alone to the activity or the whole three together or in pairs do it.

(73) Hobbs and Rhodes and Barnes lifted the rock.

(Borsley 2005: 468)

Borsley (2005) suggests possible structures and concludes that having more than two conjuncts in a structure like the one proposed by Munn (1987) creates several problems. This issue related to collective or distributive interpretation of the conjunct might need, though, an implementation from a semantic point of view that I will not be taking into consideration in this work.

Another aspect to look at is that in these approaches, coordination is considered to have features. Johannessen (1998), by looking crosslinguistically at the morphology of case used on both conjuncts, identifies unbalanced coordination, where the expected case appears only on one of the two conjuncts, as seen in 2.2.1, and extraordinary balanced coordination, in which both conjuncts show unexpected case. We can see an example of each type in (74a) and (74b), respectively. In (74a), only the second conjunct shows unexpected case, while in (74b) both conjuncts do. 
(74) a. [Han og meg] var sammen om (Norwegian) det. he.NOM and me.ACC were together about it 'He and I were in it together.'

(Johannessen 1998: 8)

b. [Them and us] are going to the game together.

(Stalke 1984: 360) cited by Johannessen 1998)

Relying on this data, Johannessen (1998) claims that features can be inherited by the specifier via spec-head relation. As for the complement, it can get default features or it inherits them from the specifier. For Zoerner (1995), instead, features of conjuncts percolate to \&P. As pointed out by Progovac (1998), Johannessen's approach is more economic and follows relations already established in syntax such as the spec-head relation.

Moreover, Johannessen considers that the input of any \&P is a propositional structure that undergoes deletion and sharing. As seen before in (63), though, there are sentences that cannot be assumed to have $\mathrm{CP}$ coordination as underlying structure, since the reduced version does not present the same meaning as the full one (e.g., Louise rode bicycles and George rode bicycles).

As for the coordination of different categories, the use of \&P does not create any problem in relation to that, but as pointed out by Zhang (2010), there is no constraint disallowing coordination of unlike categories. As we will see for LSC in 3.2, this does not hold cross-linguistically. In LSC, in fact, we will see that only categories selected by the verb can be coordinated, creating a constraint in the category selection. Moreover, Zhang (2010) claims that even if coordinators are considered to be heads, this does not mean that they 
can form an independent functional category. Zhang (2010) also points out more arguments against the existence of $\& \mathrm{P}$. The first one states that the creation of a new syntactic category needs to be based on the existence of a new syntactic distribution of elements. A coordination structure does not fit this description since it does not contribute to any specific position in the sentence of the elements. Taking a transitive verb like compare, it selects plural nominals that can either be in the form of a plural NP or two singular NPs coordinated. For the second argument, Zhang (2010) looks at the categories that can be coordinated. For cases in which two constituents of the same category are coordinated, she claims that the category of the structure takes the category of the singular conjuncts. She does not mention cases where the two constituents are for example an NP and a CP. In this case, her argument does not hold. Later, in section 2.2.4.2.4, we will see that Zhang does not require any special category for coordination even when the conjuncts have different categories. For the author, it is always the category of the first conjunct that determines the category of the whole coordinated structure. Still, though, the category of the first conjunct cannot represent the one of the two so there is no reason why not using \&P in these cases. Finally, in another argument, Zhang states that unbalanced coordination does not prove that the coordinator and is a case-related functional category that assigns case features, as defended by Johannessen (1998) and Zoerner (1995). Zhang's justification is based on the fact that in case of non-nominal coordination, the derivation would crash since non-nominal conjuncts do not have case features. 
Let us look now into the second proposal that tries to account for coordination where each conjunct is the complement of its own $\& \mathrm{P}$.

\subsection{Each conjunct is the complement of its own \&P}

A different proposal by Collins (1988) uses CONJP as marker of conjunction phrase and instead of having specifier and complement as positions for the conjuncts, each conjunct is a complement of its own conjunction phrase CONJP, as represented in (75).

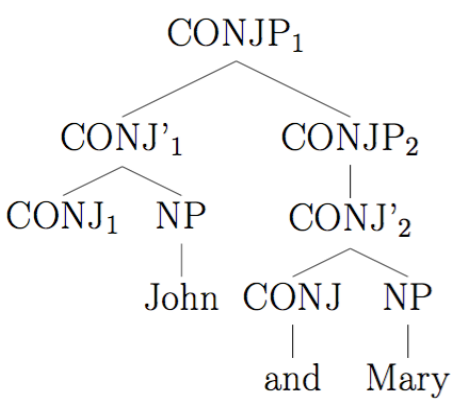

Differently from Zoerner (1995) and Johannessen (1998), recursion takes place in the specifier position, which is to the right of CONJ'. Moreover, there are as many \&Ps as there are conjuncts. For Zoerner and Johannessen, instead, the complement does not project another $\& \mathrm{P}$. The need of having a CONJP for each conjunct is due to the fact that it is possible to have a conjunction adverb per conjunct, like in (76). 
(76) Perhaps John, maybe Mary, and certainly Bill went to the store.

(Collins 1988)

Moreover, Progovac (1998: 4) points out that this structure would capture well conjunct doubling as in (77), repeated here from (49a).

(77) Sono arrivati (e) Anna, (e) Roberto, e Laura. (Italian) are arrived and Anna and Roberto and Laura. "Anna (and) Roberto and Laura have arrived."

Another problem pointed out in Progovac (1998) is that the head of the higher CONJP is always empty. Moreover, she notices that the spec-head relation of feature sharing is not invoked. She states, though, that the spec-head mechanism suggested by Johannessen (1998) is very effective, since it is very important for subcategorization, assuming that the features of the first conjunct in the specifier are assigned to \&P by spec-head agreement.

Let us look now at the third proposal that we will consider, by Munn (1993).

2.2.4.2.3 Conjuncts are adjuncts and coordinators are heads of \&P

The third proposal we are going to consider is given by Munn (1993), who assumes that some conjuncts are adjuncts. In (78), the Boolean 
Phrase (BP), used to refer to the conjunction phrase, is adjoined to $\mathrm{NP}_{1}$.

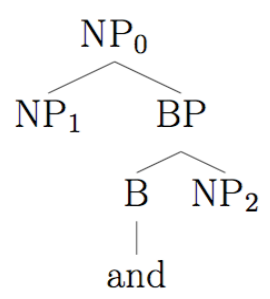

Munn (1993) assumes that conjunction must adjoin to the first conjunct at LF. This, as pointed out by Progovac (1998), would create problems in the prosodic grouping of coordination with the conjuncts. In Kayne (1994), instead, the first conjuncts are adjuncts to the last \&P because, in his antisymmetry approach, adjuncts cannot appear on the right. His structure, though, is anyway hierarchically parallel to Johannessen's.

In the next subsection we will present the last analysis in our review, by Camacho (1997) and Zhang (2010).

\subsection{Conjuncts are heads but not of \&P}

The last non-flat structure that needs to be taken into account is the option of having conjunction as a head but not of \&P. As we saw in section 2.2.2, it is possible to coordinate different categories. Camacho (1997) and Zhang (2010) suggest, then, that in a multiple specifier structure for coordination, the category of the phrase that conjunction is head of is determined by the category of the first conjunct. In (79) there are the structures they propose respectively. 
(79)
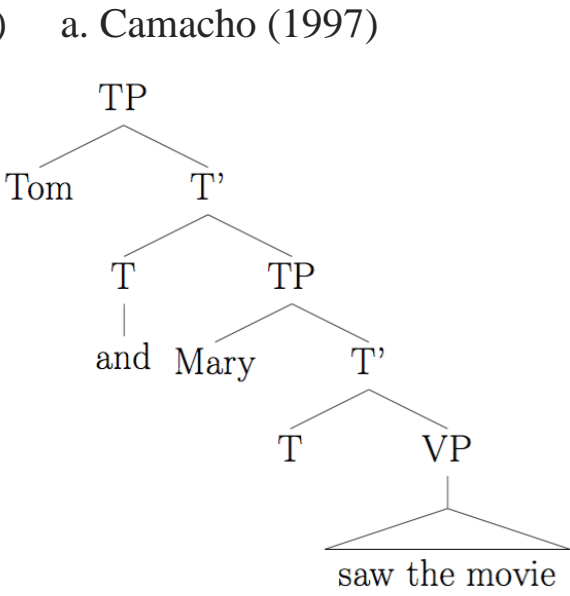

b. Zhang (2010)

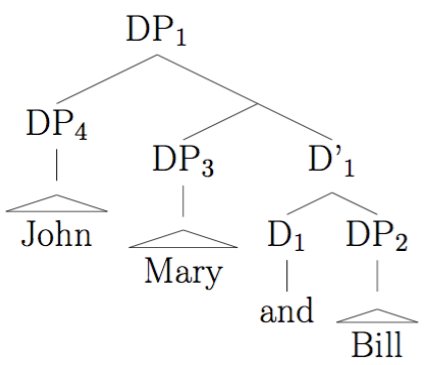

As presented in section 2.2.4.2.1 above, Zhang (2010) gives several arguments against $\& \mathrm{P}$, and in favor of assigning no special category to coordination (see also Büring 2002). Her arguments against a flat structure are related, (i) to the need of being able to represent grouping when having more than two conjuncts and, (ii) to the fact that the category of a coordination structure is never determined by non-final conjuncts. The former argument is exemplified in (80) where it is clear that it is the last conjunct that hosts the coordinator. As for the latter argument, the examples in (81) mark the contrast between non-final and final conjuncts supporting the need for a nonflat structure and for the fact that coordination does not need any special category.

(80) a. apple, oranges and bananas.

b. *apple, and oranges, bananas. 
(81) a. You can depend on my assistance, John's cooking skill, and that Mary will be on time.

b. *You can depend on my assistance, that Mary will be on time, and John's cooking skill.

c. *You can depend on that Mary will be on time, my assistance, and John's cooking skill.

(Zhang 2010: 50)

Progovac (1998), though, points out some arguments against this approach. First, in the representation in (79a) the two NPs Tom and Mary do not form a constituent. This was already noticed by Camacho (1997) and it constitutes a problem for agreement, binding and other constituency effects. Second, the lack of a special category for coordination goes against the trend in research, where a head needs its own category and therefore that should be the case also for coordination.

Finally, we present a different type of analysis, a multidimensional approach proposed by Goodall (1987), followed also by Muadz (1991), Moltmann (1992) and te Velde (1996, 1997). This analysis assumes that coordination is the union of two 2D phrase markers being merged together if belonging to an identical node. Thus, parallel levels are involved, as it is possible to see in (82b), in a multidimensional structure, representing the sentence in (82a). 
a. Jane and Alice saw Bill.

b.

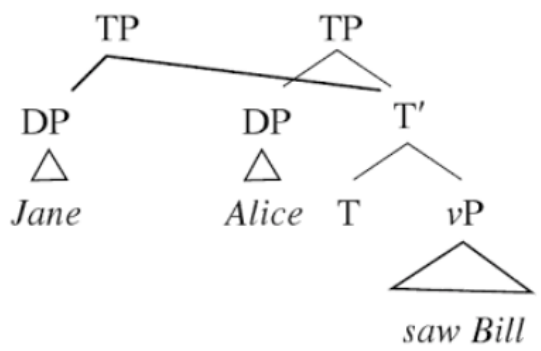

(Goodall 1987: 23)

As for linearization, conjunction is inserted at Phonological Form (PF). Progovac (1998) remarks, in favor of this approach, that conjuncts do not c-command each other being on different levels but, as seen till now, it is a property that needs to be taken into account to justify cases of binding into the second conjunct. Moreover, the structure in (82) cannot account either for asymmetries between the conjuncts, cases like (83), where the order of conjuncts is not reversible.

(83) a. John read the book and quickly.

b. *John read quickly and the book.

(Progovac 1998: 6)

None of these structures gives a final answer to the issue of providing a proper representation for coordination, but they all contribute to make the whole picture clearer.

After presenting data that are mostly related to conjunction, we will illustrate next the analysis in the literature for disjunctive and adversative coordination. 


\subsubsection{Disjunctive and adversative coordination}

In the previous sections we presented the main analyses that have been proposed in the literature for conjunction. For disjunctive and adversative coordination not much syntactic work has been done but in this section we will present some of the considerations that have been made on these two structures that will be useful in the analysis of these two types of coordination also for LSC in chapter 3.

\subsubsection{Disjunctive coordination}

Disjunctive coordination has been mainly studied from a semantic point of view, looking for example at scalar implicatures or at the scope of element such as either or or and their interpretation in affecting the conjuncts. In the '80, Partee and Rooth (1982) discussed the properties of disjunction in English and concluded that or has the properties of a scope-bearing element. Larson (1985) worked later on how so called "scope indicators" such as either or whether affect the interpretation of the scope of disjunction. For Larson (1985), the scope of $o r$ is assigned depending on the syntactic movement of either, whether or the phonologically null indicator when only or is used. Taking into consideration examples with either like the ones in (84), we can see that (84a) and (84b) have the same meaning, with three apparent readings available, following Partee and Rooth (1982). (84c), instead, only shows two of them. In $(84 a, b)$, it is possible to have a de dicto reading in which Mary is looking for a servant and this would be satisfied if the condition of this individual $\mathrm{x}$ being a 
maid or a cook is met. The second reading is a de re reading in which "for some x, a maid or a cook, Mary is seeking for x". The last one is understood as a disjunction reduction of the clauses assuming to interpret (84a) as "Mary is looking for a maid or Mary is looking for a cook". In (84b), as in (84a), it is possible to find these three readings. According to Larson's judgments, instead, in (84c), the de $r e$ reading is missing.
a. Mary is looking for a maid or a cook.
b. Mary is looking either for a maid or a cook.
c. Either Mary is looking for a maid or a cook.

(Larson 1985: 220)

It is possible to see other effects produced by the position of either in more complex sentences like the ones in (85).

(85) a. Scherlock pretended to be looking for a burglar or a thief.

b. Scherlock pretended to be looking either for a burglar or a thief.

c. Scherlock pretended to either be looking for a burglar or a thief.

d. Scherlock either pretended to be looking for a burglar or a thief.

(Larson 1985: 221)

In (85), (85a) and (85b) have the same meaning, showing the three readings presented above. In (85c), instead, or is interpreted inside 
pretend but outside look for, while in (85d) it is interpreted outside the scope of both verbs. The conclusion drawn by Larson (1985) is that when either is displaced from or, either must take clausal scope. Larson's generalization is explained also in syntactic terms, but due to the fact that it does not apply to Minimalism representation, I will it leave aside, considering only the interesting facts related to scope. Larson also looked at whether and its interaction with scope of disjunction, but I will not go through its analysis because in LSC this element is not present and the data for English will not be relevant. Either, with the same properties that we see in English, does not exist either. As we will see in 3.1.2, LSC has several elements that can convey an exclusive reading in disjunction but they are mainly found in sentence final position and they are not naturally displaced in different positions in the sentence in order to check its scope in relation with $o r$.

For the analysis of disjunction in LSC we will follow Davidson's (2013) analysis for American Sign Language (ASL), which will be presented in 2.3.4. Therefore, I shall not present any more background on disjunction in spoken languages but I suggest the reader to consult Han and Romero (2014) for an interesting study on disjunction in interaction with information structure.

We will look next at one of the main syntactic analyses proposed for adversative coordination that will be relevant for the analysis of this type of structure in LSC. 


\subsubsection{Adversative coordination}

In 2.1.1 we identified, among the different types of coordination, adversative coordination as well, which is usually expressed through the use of the connector but. In 2.1.4 we classified three types of contrast that can be expressed in coordination and two of them are realized in adversative coordination: corrective and counterexpectational. We can see an example of each one in (86), repeated from $(23 b, c)$.

(86) a. Peter is not studying in his room, but he's playing in the garden.

(Mauri 2008: 130)

b. John is tall but he's not good at basketball.

In (86a), corrective adversative coordination is expressed through the negation of the first conjunct and its substitution in the second one. In (86b), instead, where we have a case of counterexpectational adversative coordination, a presupposition in the first conjunct is not supported in the second one. Repp (2009) schematically expresses it as in (87).

(87) $\quad p$ but $q$ corresponds to $p$ and therefore $\neg q$, but actually $q$.

(Repp 2009: 85) 
Authors such as Vicente (2010) analyze these two types from a syntactic point of view, the one showing corrective but in (88a) and another one with counterexpectational but, in (88b). He also considers data from Spanish that I will not be fully reporting here.

a. Amanda ate three apples but one banana.

b. The girl is tall but no good at basketball.

(Vicente 2010: 382)

Vicente (2010) proposes a different analysis for each type of but. For the corrective one he assumes the presence of clausal coordination plus ellipsis, supporting Merchant's (2001) PF deletion account in which the remnant in the second conjunct moves to the left periphery of the sentence and the given material gets deleted. For the example in (88a) we can see the representation in (89a). In cases of counterexpectational but like the one in (88b), instead, NP coordination is at play, analyzed as in (89b). Combining the two proposals, Vicente states the following in (90).

(89) a. [Amanda ate three apples] but [[one banana $]_{i}\left[{ }_{I P}\right.$ Amanda ate $\left.\left.t_{i}\right]\right]$.

b. The girl is [tall] but [no good at basketball]. (adapted from Vicente 2010: 382)

(90) The syntax of adversative coordination (Vicente 2010: 413)

a. Corrective but always requires its conjuncts to be full clauses. 
b. Counterexpectational but allows its conjuncts to be smaller than clauses.

In order to get to this conclusion, where corrective but is always used in clausal coordination plus ellipsis, while counterexpectational can also be present in structures smaller than a clause, Vicente (2010) looks at scope of negation, preverbal subject coordination, attributive adjective coordination, first conjunct agreement, locality effect and connectivity. Here, though, we are going to present only the tests that will be relevant for the LSC discussion (see 3.3.3).

Let us start presenting the data for the scope of negation. As we can see in (91), with corrective but the scope of negation is restricted only to the first conjunct. It is possible to represent (91a) as in (91b), following Munn (1987) for the coordination structure and using as label for coordination phrase butP. With counterexpectational but in English, instead, the reading of negation is ambiguous between a wide and narrow scope over the conjuncts, as in (92a). Spanish data could help clarify this ambiguity since it has two different words for the two but: sino for the corrective (92b) and pero for the counterexpectational (92c). The counterexpectational but in (92c), then, is used in subclausal coordination of two APs, which means that it is not the case that Susana is both poor and honest, but she can be only one of the two.

(91) a. Gabriel didn't drink beer but champagne.

$[(\neg p) \wedge q]$ 
b.

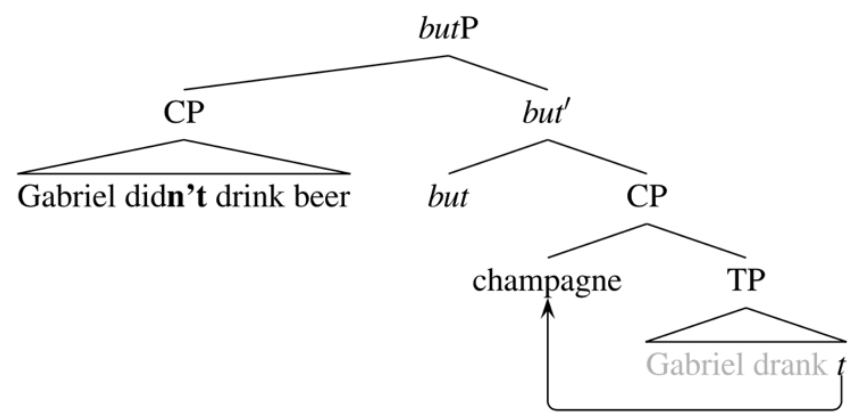

(Vicente 2010: 386,387)

(92) a. Susan is not poor but honest.

Ambiguous: $[(\neg \mathrm{p}) \wedge \mathrm{q}]$ and $[\neg(\mathrm{p} \wedge \mathrm{q})]$

b. [ $C P$ Susana no es pobre] sino [ $C P$ honesta].

(Spanish)

Susana not is poor but honest

$[(\neg \mathrm{p}) \wedge \mathrm{q}]$

c. Susana no es [AP pobre pero honesta].

Susana not is poor but honest

$[\neg(\mathrm{p} \wedge \mathrm{q})]=[\neg \mathrm{p} \vee \neg \mathrm{q})]$

(Vicente 2010: 405,406)

Another argument used by Vicente (2010) consists in looking at preverbal subject coordination. Since corrective but needs clausal coordination, this type of structure is not available, but it is with the counterexpectational but having the two NPs coordinated. We can see an example for each but, in (93a) for the corrective one and (93b) for the counterexpectational one. 
a. * Two mathematicians but seven astrophysicists didn't get their papers published.

b. [DP One single neurosurgeon but at least three cardiologists] will take part in this operation.

(Vicente 2010: 387, 406)

Finally, the main argument used by Vicente (2010) to support a clausal coordination plus deletion account for corrective but is based on Merchant's (2001) test in which island sensitivity gets checked. We will give more details about this test in chapter 4 when talking about ellipsis (see 4.1.1.2). The crucial point of this test consists of the fact that if there is sensitivity to islands, it means that the elliptical site is not empty but there is structure detected. We can see an example in (94a) where the sentence is ungrammatical because Cassandra, which is a focalized element, cannot be extracted to reach the left periphery of the sentence. This piece of data justifies the presence of clausal coordination with corrective but. In (94b), instead, we can see that with counterexpectational but there is no island violation due to the fact that there is no clausal coordination and no ellipsis.

(94) a. * I didn't leave the party [\{after/because $\}$ Amy started telling bad jokes] but [Cassandra]i [I left the party [\{after/because $\}$ ti started telling bad jokes]]. 
b. I complained to the director of the hospital [because one single surgeon but at least three unqualified students took part in the operation].

(Vicente 2010: 395, 409)

Vicente (2010) applies also other tests with attributive adjective coordination, first conjunct agreement and connectivity. The first two lead to the same conclusion we arrived at with the tests presented here. The last one, instead, shows similar behavior between corrective but coordination and counterexpectational since in both structures a pronoun in the second conjunct can be bound by a preceding quantifier. I shall not enter the explanation of these tests since they cannot be applied to LSC due to language specific properties, like in the case of agreement. Moreover, the little research on the behavior of quantifiers in LSC will not make effective the application of the connectivity test. I refer the reader to Vicente (2010) for more information about the tests applied.

In his analysis, though, Vicente (2010) focuses only on cases of corrective but where only one element is left in the second conjunct, realizing cases of ellipsis. Examples such as the one in (95) are not taken into consideration.

(95) Marina is not studying in her room, she's playing in the garden.

(Mauri 2008: 130) 
An instance of corrective but in a sentence like the one in (95) cannot be accounted with an analysis that fits ellipsis data. It is anyway interesting to notice the support that Vicente (2010) gives to Merchant (2001) and to the deletion analysis for ellipsis in general. To the deletion approach we will be giving further support by examining another type of ellipsis in LSC, gapping (see chapters 4 and 5). As for structures like (95), it is necessary to point out that for cases of corrective adversative coordination not appearing in ellipsis, the analysis proposed by Vicente (2010) does not apply. It is true, though, that probably no special mechanism is involved to account for (95) due to the clear nature of coordination of two clauses where the subject of the second clause is coreferential with the one in the first one. More interesting observations might be implemented looking at LSC in 3.3.3, where the contrast between the two conjuncts is marked also in their location in space. For other cases of adversative coordination in ellipsis, I refer the reader to Repp (2009).

\subsubsection{Summary and conclusions}

In this first part of chapter 2 about spoken languages, we looked at the structural and functional properties that characterize coordination cross-linguistically. In the first part we mainly focused on how coordination is marked structurally and how the semantics of the relation between conjuncts is affected. In the second part, instead, we went through the main aspects that need to be taken into consideration in order to provide a syntactic structure for coordination. We then analyzed the main proposals that we can find 
in the literature that give a syntactic account for coordination considering conjunctive, disjunctive and adversative coordination. It is clear, from the arguments that have been presented, that it is necessary to have a non-flat structure and also an asymmetric one. Even in cases of semantic symmetric coordination where conjuncts express simultaneity or contrast, it is still possible to have asymmetry if a possessive pronoun is used in the second conjunct referring to the referent in the first one, for example. As for the category to use for coordination structure, the arguments presented give valid reasons to either choose \&P or the category of the first conjunct. I find the need of giving a category to each head very important and therefore also to the one of coordinators. For the representation, this category should be $\operatorname{Co}(\mathrm{P})$, which includes conjucntive, disjuntive and adversative coordination. Moreover, while for spoken languages there is no hint coming from prosody or syntax that tells us that the structure that will be produced is coordination, this is possible in Sign Languages. Therefore, as we will argue in chapter 3 for LSC, it is even more plausible to have a specific category and a phrase structure such as CoP for coordination.

\subsection{Coordination in sign languages}

In this section we are going to introduce the main studies that contribute to the understanding of coordination in sign languages (SLs). We are going first to look at the main properties of SLs and we will then focus on the main strategies used to express the different types of coordination in American Sign Language (ASL), Hong 
Kong SL (HKSL), Finnish SL (FinSL), Turkish SL (TID), British SL (BSL) and SL of the Netherlands (NGT). In 2.3, we will go through the tests used to diagnose coordination in opposition to subordination and finally, in 2.3.4 we will present the only theoretical account that have been proposed for coordination in SLs, by Davidson (2013).

The goal of this section is to give a cross-linguistic background to coordination in SLs before introducing the data for LSC, in chapter 3.

\subsubsection{Types of coordination in sign language}

Coordination, also in SLs, involves the combination of two or more constituents that can be NPs, VPs, TPs or CPs. These elements can be linked overtly or using juxtaposition, where NMMs play an important role. As we will see in the next subsections, NMMs are very often crucial in the expression of the different types of coordination. Most of the data in the literature of SLs focus on sentential coordination rather than NP coordination; therefore, due to the lack of data on NP coordination and to the fact that the analysis of coordination in LSC will focus on sentential coordination, no data on NP coordination will be presented. It is important to take into account, though, that the main strategies used in sentential coordination can be extended also to NP coordination, as attested at least for ASL (Davidson 2013).

In SLs, as in spoken languages, there are three main types of coordination: conjunctive, disjunctive and adversative coordination. In order to distinguish them, similar strategies cross-linguistically 
have been found, using both manual and non-manual markers. Generally, coordination in SLs is expressed asyndetically using only NMMs and often causing ambiguity in the interpretation of conjunction or disjunction. Prosody and lexical cues, as in the case of ASL (Davidson 2013), help to disambiguate them. In other SLs, like FinSL (Jantunen 2016), instead, disjunction is predominantly expressed manually and consequently the use of only NMMs leads to interpreting conjunction. Adversative coordination, instead, is in general mainly expressed manually.

\subsubsection{Expression of coordination in SLs: manual and non-manual markers}

In this section we will see how each type of coordination is expressed cross-linguistically considering data from ASL, HKSL, Auslan and FinSL.

\subsubsection{Conjunctive coordination}

As anticipated, conjunctive coordination also called conjunction, in SLs is generally expressed only through the use of NMMs. Crosslinguistically, a movement of the upper part of the body, head and torso, is involved. Moreover, a prosodic break is performed between the conjuncts, usually marked by eye blinking, a marker of constituency and clause boundaries (Wilbur 1994; Herrmann 2010; Pfau \& Quer 2010). In FinSL, the movement of the head over the conjuncts is identified as side-way tilts (Jantunen 2016) and in 
HKSL, instead, as head turns spreading in opposite directions (Tang \& Lau 2012). For ASL, Davidson (2011) glosses with COORD-SHIFT the movement of the torso towards opposite directions. Moreover, an extended head nod between the conjuncts is generally produced on the last sign on each conjunct which, for Liddell (1980, 2003), marks in ASL the existence of a state or process. The same NMMs are also found in HKSL by Tang \& Lau (2012) who claim that they are used to assert a proposition. The combination of body and head movements is used in ASL (Padden 1988), HKSL (Tang \& Lau 2012) and Auslan (Johnston \& Schembri 2007) to express simultaneous and sequential events. In (96a) and (96b) we can see an example from HKSL of sequential and simultaneous events, respectively.

$\mathrm{eb}$ $\mathrm{eb}$

a. MOTHER DOOR CL:UNLOCK-DOOR, CL:PUSH-OPEN, eb

$\frac{\mathrm{hn}}{\mathrm{CL} \cdot \mathrm{ENTER} \text { HOUSE. }}$

CL:ENTER HOUSE.

'Mother unlocked the door, pushed it open (and) went inside.'

b. BOY $\frac{\mathrm{eb}}{\operatorname{IX}_{3} \operatorname{SIT}_{\mathrm{a}}}$, CHIPS, SODA, EAT-CHIPS, DRINK-SODA, ht right EAT-CHIPS, ....

'The boy is sitting here, he is eating chips (and) drinking soda.' 
Another strategy to express simultaneous events takes advantage of SL simultaneous modality where each hand can express an event at the same time. In (97) we can see an example from FinSL where hand $1(h 1)$ and hand $2(h 2)$ correspond to the two manual articulators that are combined with facial expressions indicating a puzzled state of the signer. Similar data are reported also for HKSL (Tang \& Lau 2012).

"the signer looks around in a puzzled manner"
h1: CL-Vbent-"sit"-2 right
(FinSL)
h2: CL-Vbent-"sit"-2 left
i. 'The boy and the dog sit and look around in a puzzled manner.'
ii. 'The boy sits and looks around in a puzzled manner and the dog sits and looks around in a puzzled manner'.

(Jantunen 2016: 225)

Despite the fact that conjunction is mainly expressed via NMMs, another way to introduce conjuncts, especially if they are more than two, is via list buoys. List buoys, as called by Liddell (2003), are a SL-specific form used especially in listing, either of NPs or bigger constituents. The signer produces a bimanual sign in which the nondominant hand holds a stationary handshape representing the number of the referents that will be introduced while the dominant one points to the fingers on the non-dominant hand selecting a finger per entity with an extended index handshape. The list buoy for each referent is usually signed at the beginning of each conjunct. For ASL, Davidson 
(2011) glosses list buoys with COORD-L when there are two referents in the sentence, as we can see in $(98)^{9}$.

HAPPEN? COORD-L 1 [POSS ${ }_{a}$ PARENTS WILL BUY

(ASL)

$\left.\mathrm{POSS}_{a} \mathrm{CAR}\right] \mathrm{COORD}-\mathrm{L}_{2}$

[IX $a$ WILL TRAVEL].

'What will happen? Her parents will buy her car, and (then)

she will travel.

(Davidson 2011: 72)

Other manual markers that can be found in conjunction in SL are described for FinSL where, in sentences with multiple coordination, it is possible to find the signs ALSO or PLUS in combination with the NMMs typical of conjunction. Languages like Auslan do not have a sign corresponding to the English and and in HKSL, if used, it would result in a structure that follows that of spoken Chinese.

As described till now, conjunction is mainly expressed asyndetically in SLs; only BSL shows a preference for syndetic coordination, as pointed out by Waters \& Sutton-Spence (2005). Asyndetically, conjunction tends to be ambiguous between expressing conjunction or disjunction, but context, prosody and lexical cues are crucial in the interpretation of either one or the other. Let us look, then, at how disjunction is expressed and disambiguated from conjunction.

\footnotetext{
${ }^{9}$ The gloss COORD-L will be used only in Davidson's examples in order to respect her notations. In the other examples, list buoys will be glossed as LISTX, with $x$ varying depending on the number selected on the finger of the non-dominant hand.
} 


\subsubsection{Disjunctive coordination}

Disjunctive coordination, also called disjunction, is expressed in the same way as conjunction in all the SLs that predominantly use NMMs to express them. As anticipated in 2.3.2.1, these two structures are ambiguous and only the context, the prosody or lexical cues can help distinguish them. For ASL, Davidson (2013) explains that disjunction can be expressed using either only NMMs, glossed by her as COORD-SHIFT due to the movement of the torso, or list-buoys (COORD-L in her notation). NMMs such as squint and bit lip help to support a disjunctive reading while for conjunction it is more common to find neutral eyebrow and a head nod. NMMs alone, though, cannot contribute to distinguish the two types of coordination. A necessary extra element consists in adding at the end of the sentence "I don't know which" for an exclusive reading, as in (99a), or "maybe both" for an inclusive one, as in (99b). An alternative question like the one in (99c) is another option to express disjunction without having an inclusive or exclusive reading. In (99d) we can see that the exclusive use of the NMMs COORD-SHIFT to express disjunction will make the sentence degraded due to the ambiguity with conjunction. In the examples below, COORD-SHIFT is used but the same data apply to examples with COORD-L (see Davidson 2013: 4: 7, 8).

a. [POSS $a$ PARENTS WILL BUY POSS $a$ CAR $]_{b}$

COORD-SHIFT [IX $a$ WILL TRAVEL $]_{c}$ (DON'T-KNOW WHICH).

'Her parents will buy her car or she will travel, I'm not sure which.' 
b. $\left[\operatorname{POSS}_{a}\right.$ PARENTS WILL BUY POSS $_{a} \mathrm{CAR}_{b} \mathrm{COORD}_{\text {-SHIFT }}\left[\mathrm{IX}_{a}\right.$ WILL TRAVEL $]_{c},($ MAYBE BOTH).

'Her parents will buy her car or she will travel, maybe both.'

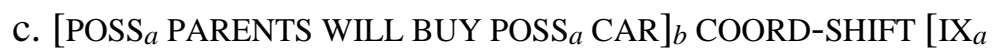
WILL TRAVEL $]_{c}$, WHICH?

'Will her parents buy her car, or will she travel?'

d. ? [POSS $a$ PARENTS WILL BUY POSS $a$ CAR $]_{b}$ COORD-SHIFT [IX $a$ WILL TRAVEL $]_{c}$ ?

'Will her parents buy her car, or will she travel?'

(Davidson 2013: 4:10)

In HKSL, instead, slightly different NMMs are found in conjunction and disjunction. While in conjunction only head nod is used, in disjunction we find the combination of head nod and head and body turns. Moreover, lexical cues such as EITHER are used, as we can see in (100).
eb eb (100) $\mathrm{IX}_{1}$ GO-TO BEIJING, $\left(\mathrm{pro}_{1}\right) \frac{\mathrm{hn}+\mathrm{bt} \text { right }}{\text { TAKE-A-PLANE, }}$ eb eb
eb hn+bt left

(HKSL)

TAKE-A-TRAIN, EITHER DOESN'T-MATTER.

'I am going to Beijing. I will take a plane or take a train. Either way, it doesn't matter.'

(Tang \& Lau 2012: 343) 
FinSL, instead, predominantly uses the manual marker OR to express disjunction, in combination with a change in the upper-body location where the body swings from left to right. Also in BSL and ASL it is possible to use an overt marker: the sign OR or the finger-spelled $O-R$.

While conjunction and disjunction are mainly expressed asyndetically, adversative coordination is very often found with the manual marker BUT. Let us see the properties of this third type of coordination in the next section 2.3.2.3.

\subsubsection{Adversative coordination}

Adversative coordination is generally expressed using the manual marker BUT, like in Auslan, ASL, BSL and FinSL. We can see an example from Auslan in (101).

(101) K-I-M LIKE CAT BUT P-A-T PREFER DOG.

(Auslan)

'Kim likes cats but Pat prefers dogs.'

(Johnston \& Schembri 2007: 213)

HKSL is the only SL reported where adversative coordination can be expressed only through the use of NMMs, as in the example in (102). Tang \& Lau (2012) claim that if manual markers are absent, NMMs are more prominent. In (102), we observe use of juxtaposition in combination with head turns and forward and backward body leans, other than head nod and eye blinks, as found also in other types of coordination. 
eb

(102) EXAM COME-CLOSE, RUTH DILIGENT

(HKSL)

eb

eb

$\mathrm{hn}+\mathrm{ht}+\mathrm{bt}$ forward $\frac{\mathrm{hn}+\mathrm{ht}+\mathrm{bt} \text { backward }}{\mathrm{D}}$

DO-HOMEWORK, HANNAH LAZY WATCH-TV.

'The exam is coming close; Ruth is diligently doing her homework (but) Hannah is lazy and watches TV.'

(Tang \& Lau 2012: 344)

Eye blinking, as mentioned in 2.3.2.1, is a clause boundary marker, and in FinSL it can be used to establish the position of the coordinator with respect to the conjuncts. Coordinators such as ALSO, PLUS, OR or BUT are produced between the two conjuncts and an eye blink produced before the coordinator makes clear that in FinSL the coordinator belongs to the second conjunct, realizing prepositive coordination (see 2.1.3). Not much is reported for SLs in relation to this, but for LSC we will see in 3.3.4 that the position of the coordinator is crucial to determine the directionality of the syntactic structure used to analyze coordination, as already introduced in 2.2.1.1 when referring to Zhang (2010) for spoken languages.

In the next section 2.3.3 we will review other syntactic properties of coordination that will be tested also on LSC in order to be able to give a proper syntactic analysis of coordination that can be extended to other SLs, too. 


\subsubsection{Tests to identify coordination (vs subordination)}

The fact that SLs in general lack overt complementizers to mark the presence of clausal arguments makes it more difficult to tell apart this structure from coordination. Tang \& Lau (2012), though, propose three main tests to diagnose coordination: i) extraction, more precisely across-the-board (ATB), ii) gapping and iii) scope of yes/no questions and negation. With the purpose of detecting subordination, van Gijn (2004) suggests some tests, among which one can also be applied to identify coordination, that is, the distributional dependency test. It consists in looking at the dependency between sentences: if the two sentences, even if related, can stand by themselves, they are then two conjuncts. Göksel \& Kelepir (2016), with the same purpose, use prosody assuming that the presence of prosodic boundaries between clauses can be a diagnostic for coordination. In the next subsections we will see these tests in detail. They will also be applied in 3.2.3 for LSC in order to determine the properties of potential coordinated structures in this language.

\subsubsection{ATB extraction}

In section 2.2.3 we saw that coordination is sensitive to the Coordinate Structure Constraint (CSC) by Ross (1967), which states that it is not possible to move a conjunct or extract an element from it, as we saw in example (56) for wh-extraction in spoken languages. This applies also to SLs. Tang and Lau (2012) for HKSL show that 
the CSC is respected. In (103) we can see that in HKSL it is not possible to have wh-extraction of an element out of a conjunct.

$$
\begin{aligned}
& \text { *YESTERDAY DAD PLAY _ _ }, \text { EAT } \\
& \text { COW`CL:CUT-WITH-FORK-AND-KNIFE WHAT }_{\mathrm{i}} \text {. } \\
& \text { Lit. '*What did daddy play and eat steak?' }
\end{aligned}
$$

(Tang \& Lau 2012: 435)

As reported by Ross (1967), CSC can be violated in two ways: in asymmetric coordination and using ATB movement, extracting the same element from both conjuncts (for more discussion on this, see section 2.2.3). For the first case there is no data in the literature of SLs, but there is for the second one. As we can see in (104), though, ATB wh-extraction cannot be realized in HKSL. In order for the sentence to be grammatical, it is necessary to repeat the wh- element WHAT in both conjuncts, as in $(104 b)^{10}$.

$$
\text { a. *MOTHER LIKE } \mathrm{t}_{\mathrm{i}} \text {, FATHER DISLIKE } \mathrm{t}_{\mathrm{i}}, \frac{\mathrm{wh}}{\mathrm{WHAT}_{\mathrm{i}}} \text { ? }
$$

Lit. 'What does mother like and father dislike?'

\footnotetext{
${ }^{10}$ For ASL, Padden (1988) considers cases of topicalization of the same element out of both conjuncts instances of ATB movement, like in (i). Also Tang \& Lau (2012) report similar data for HKSL, as in (ii). CSC by Ross (1967) does not directly include topicalization, though. For LSC, then, I will only consider whextraction.
}

(i) THAT MOVIE ${ }_{i},{ }_{b}$ STEVE LIKE $e_{i}$ BUT ${ }_{c}$ JULIE DISLIKE $e_{i}$.

'That movie $_{i}$, Steve likes $e_{i}$ but Julie dislikes $e_{i} \cdot$ ' (Lillo-Martin 1991:60)

(ii) $\overline{\text { ORANGE }}_{i}$, MOTHER LIKE $t_{i}$, FATHER DISLIKE $t_{i}$.

(HKSL)

'Orange, mother likes (and) father dislikes.'

(Tang \& Lau 2012:346) 
b. MOTHER LIKE $t_{j} \frac{w h}{\operatorname{WHAT}_{j}}$ ? FATHER DISLIKE $t_{i}, \frac{w h}{\operatorname{WHAT}_{i} \text { ? }}$
Lit. 'What does mother like? What does father dislike?'

(Tang \& Lau 2012: 346)

Tang \& Lau (2012) suggest that the impossibility of having ATB whextraction might be due to the directionality of the specifier in $\mathrm{CP}$, which in HKSL is positioned in the right periphery. Further data are required to give a clearer explanation. In section 3.2.3.1 we will see that the same constraint applies also to LSC, but more data will be provided in order to address the issue at the structural level, not just in relation to the position of the specifier of $\mathrm{CP}$, but also of the whole coordination structure itself.

Let us see next the second test proposed by Tang \& Lau (2012).

\subsubsection{Gapping}

In coordination, a typical structure that can be produced is ellipsis. While other types of ellipsis can be found also in subordination, gapping is claimed to be used only in coordination. This is the general tendency in understanding gapping, even though, as we will see in 5.2, in languages like LSC, gapping can also be expressed in subordination.

Gapping can be defined as a type of ellipsis in which a verb is elided in one, or more, of a series of conjuncts (Johnson 2014), as 
we can see in the example in (105) for English. Sticking to this explanation, Tang \& Lau (2012) use the realization of gapping as the confirmation of the production of coordination in a sentence. In (106) we can see an example of gapping in HKSL and ASL. In both languages, the verb appears to be missing in the second conjunct, realizing an SVO-SO order.

(105) John ate a doughnut and Mary, a croissant.

(106) a. HAVE WONDERFUL PICNIC. PRO.1 BRING SALAD, (ASL) JOHN BEER SANDY CHICKEN, TED HAMBURGER.

'We had a wonderful picnic. I brought the salad, John (brought) the beer, Sandy (brought) the chicken and Ted (brought) the hamburger.'

(Liddell 1980: 31)

b. TOMORROW PICNIC, IX 1 BRING CHICKEN

(HKSL)

SANDWICHES, WING, PIPPEN KENNY COLA, CONNIE CHOCOLATE

'(We) will have a picnic tomorrow. I will bring chicken wings, Pippen (brings) sandwiches, Kenny (brings) cola, (and) Connie (brings) chocolate.'

(Tang \& Lau 2012: 347)

More data on gapping will be presented in chapters 4 and 5 for spoken languages and LSC. For now, I claim that this test, given the crosslinguistic differences in the type of structure licensing gapping, cannot be used as clear diagnostic test for coordination in languages that allow gapping also in subordination, like LSC. 
Other tests can also be applied, though, as we will see in 2.3.3.3, relying on the scope of yes/no questions and negation.

\subsubsection{Scope of yes/no questions and negation (in conjunction)}

Looking at the scope of the confirmation tag in yes/no questions and negation over the two conjuncts is another diagnostic for coordination. Yes/no questions usually end with a sign that refers back to the two conjuncts just introduced in order to confirm whether they are both true or false. As we can see in (107), RIGHT-WRONG at the end of the sentence has this function. Moreover, raised eyebrows, typical of yes/no questions, spread over the final sign. Head nod and body turn, instead, mark the two conjuncts.

(107) PIPPEN BRENDA THEY-BOTH GO HORSE-BETTING.

(HKSL)

$\frac{\mathrm{hn}+\mathrm{bt} \text { left }}{\text { BRENDA WIN, PIPPEN LOSE, RIGHT-WRONG? }}$

Lit. 'Pippen and Brenda both went horse-betting. Did Brenda win and Pippen lose?'

(Tang \& Lau 2012: 348)

In (107) we can see that having RIGHT-WRONG scoping over both conjuncts shows that there is an instance of coordination. The same happens in HKSL also with negation that can scope over both conjuncts even being at the end of the two, as we can see in (108). 
(108) TEACHER PLAY SPEEDBOAT

(HKSL)

EAT COW^CL:CUT-WITH-FORK-AND-KNIFE NOT-HAVE.

'The teacher did not ride the speedboat and did not eat beef steak.'

(Tang \& Lau 2012: 348)

This test of scope is better used with conjunction than with other types of coordination. This is due to the fact that negation can also scope over only one conjunct, for example in adversative coordination. In ASL, as well as in HKSL, it is possible to have headshake spreading over one conjunct to negate it and head nod over the other to express contrast. We can see an example of that from HKSL in (109).

\section{(109) FELIX COME GLADYS COME NOT. \\ ht backward+hs+pursed lips \\ 'Felix will come (but) Gladys will not come.'}

The tests presented till now are used by Tang and Lau (2012) as diagnostics for coordination. Other authors such as van Gijn (2004) and Göksel \& Kelepir (2016) propose other tests, even if their target is subordination. Let us see these last two tests: the distributional dependency test (2.3.3.4) and presence of prosodic boundaries (2.3.3.5). 


\subsubsection{Distributional dependency test}

Van Gijn (2004), with the goal of identifying subordination in NGT, proposes several tests, among which some of those we saw above. The one that we will be considering here is the distributional dependency test, first proposed by van Valin \& LaPolla (1997). This test looks at the possibility for the clauses in a complex sentence to be independent: if the two clauses can stand by themselves, then it is a case of coordination, but if one of the two cannot be independent, then it is a case of subordination. Van Valin \& LaPolla (1997) point out, though, that to the dichotomy of coordination and subordination it is also necessary to add cosubordination, a type of sentence that shares properties with both coordination and subordination. In cosubordination, the clauses are not independent but the dependent clause is not a modifier nor an argument of a matrix clause. We will not go deeper into this aspect, even though this type of sentences needs to be taken into account when looking at cases of asymmetric coordination (see 2.1.4), where the two conjuncts show a syntactic or semantic dependency. Applying van Gijn's (2004) distributional dependency test in a sentence in NGT with the transitive verb SEE, the relation between the two clauses in (110a) is clearly one of subordination, since the first part cannot stand by itself. In (110b), as well, the two clauses are in a dependency relation, realizing subordination. Considering that the verb SEE in NGT always agrees with the object, though, it is possible to assume that (110b) can be interpreted as having a null pronoun (pro) in the object position of SEE referring to the subject of the next clause that it agrees with, as in 
(110c). In the latter case, the two clauses will be juxtaposed expressing coordination.

(110) a. INGE SEE [MARIJKE HOUSE IX-3 GO]. ${ }^{11}$

(NGT)

'Inge sees that Marijke goes home.'

b. WE-TWO SEE [ MAN IX-3i BOOK STEAL ]

'The two of us see the man steal a book.'

c. WE-TWO SEE pro $_{\mathrm{i}}$ [ MAN $\mathrm{IX}-3_{\mathrm{i}}$ BOOK STEAL]

'The two of us see $\left(\operatorname{him}_{\mathrm{i}}\right)$; the $\operatorname{man}_{\mathrm{i}}$ steals a book.'

(adapted from van Gijn 2004: 62, 63)

On the basis of the data presented for NGT, I find this test adequate to test subordination and symmetric coordination, but I do not consider it fully appropriate as a general test for coordination due to the properties of asymmetric coordination in languages. Despite being semantically symmetric, sentences can be syntactically asymmetric (see 2.1.4 and 2.2.1 for further discussion on this point).

\footnotetext{
${ }^{11}$ I adapted the glosses used by van Gijn $(2004: 62,63)$ to my notation system. The original examples are the following:

(110) a. INGE signer $\mathrm{SEE}_{\text {left }}\left[\right.$ MARIJKE HOUSE POINT front left GO.TO $_{\text {right }}$ （NGT) 'Inge sees that Marijke goes home.'

b. THE.TWO.OF.US signer SEE front $\left[\right.$ MAN POINT $_{\text {front }}$ BOOK STEAL ]

'The two of us see the man steal a book.'

c. THE.TWO.OF.US signer $\mathrm{SEE}_{\text {front }}$ pro(=man) [ $\mathrm{MAN}_{\mathrm{POINT}}$ front $\mathrm{BOOK}$ STEAL ]

'The two of us see $\left(\mathrm{him}_{\mathrm{i}}\right)$; the $\operatorname{man}_{\mathrm{i}}$ steals a book.'
} 
The last test that we are going to present in next section is by Göksel \& Kelepir (2016), who illustrate data from TiD involving the use of prosody.

\subsubsection{Presence of prosodic boundaries}

Göksel \& Kelepir (2016) look mainly at identifying subordination in TiD and to do so they compare this structure to coordination. Their goal is to detect the NMMs that indicate the presence of independent clauses and consequently coordination, in opposition to subordination where they will not be used. Göksel \& Kelepir (2016) compare the NMMs found at complement clause boundaries to those used between coordinated clauses. In their study, the ones between coordinated clauses turned out to be similar to the TP boundaries NMMs found by Gökgoz \& Arik (2011). Gökgoz \& Arik (2011) consider head-downs, eye-blinks, head-nods, head-holds and headback as possible markers of TP boundaries, but none of those were prominent with respect to the each other. Interestingly, in coordination, Göksel \& Kelepir (2016) found lean backward, comparable to Gökgoz \& Arik's (2011) head-back, on the final conjunct and head thrust, similar to Gökgoz \& Arik's (2011) headnod, on the non-final conjunct, as in (111).

(111) [[MELEK RUN MUCH LIKE] [OZAN SWIM MUCH LIKE]] 'Melek likes running very much, Ozan likes swimming very much.'

(Göksel \& Kelepir 2016:82) 
Assuming that head thrust marks a prosodic boundary, Göksel \& Kelepir (2016) consider it as a marker of TP boundary in (111), also because they noticed that head thrust does not occur between verbs and their clausal complements. Therefore, finding NMMs such as head thrust on the non-final conjuncts can be considered a test to detect coordination in a sentence, in opposition to verb-complement relation. The data presented for this test are for conjunction so they might not apply to other types of coordination in TiD.

Until now we saw the tests used to identify the presence of coordination but we will see next the analysis that have been proposed for coordination in RSL and ASL.

\subsubsection{Conjunction (vs) disjunction: analysis}

There is not much research in the formal analysis of coordination in SLs. The main contribution comes from Davidson (2013), who proposes how to disambiguate structurally conjunction and disjunction from a semantic point of view in ASL. Another indirect contribution comes from Kimmelman (2017) who looks at simultaneous events expressed via weak hand holds in RSL and uses a CoP structure to coordinate simultaneous TPs in a multidominant structure (see more in 2.3.1.1). In addition, Legeland et al. (2018) work on coordination in NGT, and examine parallelism in conjunction and disjunction (see also 1.1.4 for spoken languages). They argue that the requirement of having a parallel structure in the number of arguments contrasting in each conjunct, the "parallel structure constraint" (PSC), can be violated in NGT. If motivated by 
Information Structure through the movement of contrasting elements to the left periphery of the structure, word order of NGT can vary. Word order can then be considered highly determined by discourseconfigurational principles. I will not enter this discussion for LSC, therefore I address the reader to Legeland et al. (2018).

As explained in 2.3.2, and we will see similar data also for LSC in chapter 3, conjunction and disjunction are mainly expressed through NMMs and the context and extra lexical elements can help distinguish them. The two main strategies used to express conjunction and disjunction in ASL are COORD-SHIFT and COORD-L, but as pointed out by Davidson (2013), "it is not the case that COORDSHIFT and COORD-L each happen to be the same phonological realization of two separate items" in conjunction and disjunction (Davidson 2013:4:14). They are a single lexical item that has a setunion semantic and the conjunctive or disjunctive reading is determined via a coordination-external universal or existential operator scoping over the whole coordination structure. The context, the use of further lexical items and NMMs help disambiguate the two. NMMs such as squint and bit lip help to support a disjunctive reading, while for conjunction it is more common to find neutral eyebrow and a head nod. NMMs alone, though, cannot contribute to distinguish the two lexical elements for each type of coordination. This is also due to a general tendency in ASL to not to have productive generation of minimal pairs based on NMMs. The compositional analysis proposed by Davidson (2013) gives a single semantic for COORDSHIFT and COORD-L, assuming that each performs the function of set 
union, as we can see in (112), where each lexical item takes sets as arguments.
(112)
a. $[[$ A COORD-SHIFT B $]]=[[\mathrm{A}]] \cup[[\mathrm{B}]]$
b. $[[\mathrm{A}$ COORD-L B $]]=[[\mathrm{A}]] \cup[[\mathrm{B}]]$

In order to coordinate all types of arguments, Davidson (2013) adopts Hamblin's (1973) rules, where lexical items are mapped to the singleton set of their normal denotation, and, if necessary, to a set of more than one individual or alternative (e.g., $\{c, t\})$. Moreover, based on Alonso-Ovalle's (2006) alternative semantics analysis of disjunction, the semantic contribution of the coordinator in ASL consists in collecting alternatives. The conjunctive or disjunctive reading of this coordinator, though, depends on the presence of a quantifier that operates upon it: an existential quantifier for disjunction (113a) and a universal one for conjunction (113b).

$$
\begin{aligned}
& \text { a. } \exists P=\lambda w \cdot \exists p[p \in P \wedge p(w)] \\
& \text { b. } \forall P=\lambda w \cdot \forall p[p \in P \rightarrow p(w)]
\end{aligned}
$$

(Alonso-Ovalle 2006)

In (114) we can see the derivation of a disjunctive use of COORDSHIFT where the existential quantifier is used. Davidson (2013) also proposes a syntactic representation of the sentence where the existential quantifier is scoping over the whole structure, as in (115). In case of conjunction instead of disjunction, the universal quantifier would be used in the derivation and put in the structure scoping over the whole structure, instead of the existential one as we can see in 
(114vi, vii) for disjunction. Until (114v), the derivation for conjunction and disjunction is the same.

(114) MARY DRINK COFFEE $a$ COORD-SHIFT TEA $b$.

'Mary drank coffee or tea.'

i. COFFEE $=\{c\}$, tea $=\{t\}$ COFFEE $_{a}$ COORD-SHIFT TEA $_{b}=\{c, t\}$

ii. DRINK $=\left\{\lambda y \lambda x \lambda w \cdot \operatorname{drink}_{w}(x)(y)\right\}$

iii. DRINK COFFEE ${ }_{a}$ COORD-SHIFT TEA $_{b}$ $=\left\{\lambda x \lambda w \cdot \operatorname{drink}_{w}(x)(\{c, t\})\right\}$ $=\left\{\lambda x \lambda w \cdot \operatorname{drink}_{w}(x)(c), \lambda x \lambda w \cdot \operatorname{drink}_{w}(x)(t)\right\}$ iv. $\operatorname{MARY}=\{m\}$ V. MARY DRINK COFFEE ${ }_{a}$ COORD-SHIFT TEA $_{b}$ $=\left\{\lambda w \cdot \operatorname{drink}_{w}(m)(c), \lambda w \cdot \operatorname{drink}_{w}(m)(t)\right\}$

vi. $\exists P=\lambda w . \exists p[p \in P \wedge p(w)]$

vii. $\exists\left[\right.$ MARY DRINK COFFEE $_{a}$ COORD-SHIFT TEA $\left._{b}\right]$ $=\lambda w \cdot \exists p\left[p \in\left\{\lambda w \cdot \operatorname{drink}_{w}(m)(c), \lambda w \cdot \operatorname{drink}_{w}(m)(t)\right\} \wedge\right.$ $p(w)]]$ 


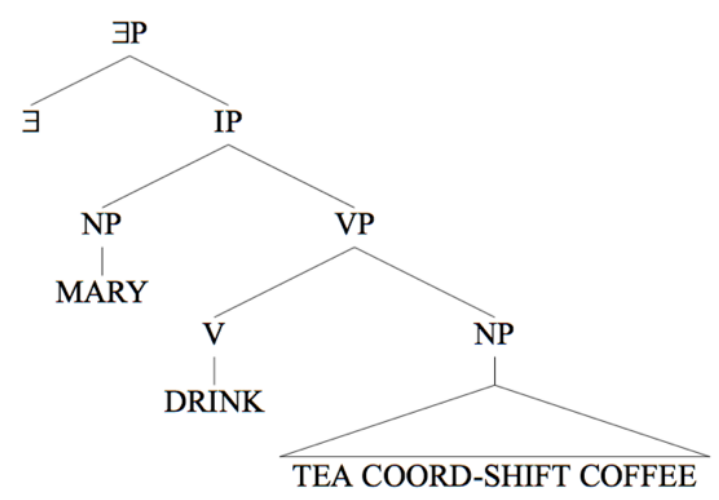

(Davidson 2013: 4:20-4:22)

In (115), the author seems to adopt XP as category of coordination, but no information about this choice is given. The important contribution of Davidson's work is not syntactic, but semantic. The presence of a quantifier scoping over the whole structure is an important tool that I think can also help to justify the presence of certain NMMs for conjunction and others for disjunction.

\subsubsection{Summary and conclusions}

In this section we looked at how coordination is expressed in SLs, identifying the manual and non-manual markers used to express it across languages. Moreover, NMMs in coordination turned out to be crucial in determining the position of the coordinator with respect to the two conjuncts. This aspect needs to be taken into consideration in the analysis of coordination also in SLs. In chapter 3 on coordination in LSC and chapter 5 on gapping, we will see that having prepositive or postpositive coordination in LSC is a crucial point in determining 
the directionality of the coordination structure and therefore in establishing the directionality of gapping.

In the second part, we presented the tests that can diagnose coordination in SLs, since there are almost no overt complementizers used to mark clearly the difference between coordination and subordination. All the tests presented need to face language specific properties and they do not necessarily apply to all types of coordination. Scope of negation, for example, can be evaluated only in conjunction. ATB wh-extraction cannot be applied to HKSL and we will see in 3.2.3.1 that LSC does not allow it, either. In comparison to spoken languages (see 2.2.3), coordination looks at first sight anomalous in SLs. Tang \& Lau (2012) attribute the ungrammaticality of ATB wh-extraction to the position of the specifier of CP in HKSL, which appears to be on the right periphery of the structure. In 3.2.3.1, though, thanks to LSC data, we will see that the ungrammaticality of ATB might be derived from a structural limitation of the coordination structure itself that affects not only whextraction, but also phenomena like Right Node Raising (RNR). For further discussion see 3.2.3.1 and 5.6.

Finally, we presented Davidson's (2013) semantic analysis to account for the presence of a conjunctive or disjunctive reading of the items used to express coordination in ASL (COORD-SHIFT and COORD-L). This quantificational approach over sets of alternatives will be useful also to account for LSC distribution of NMMs in conjunction and disjunction.

In the next sections we will first introduce the data related to how coordination is expressed in LSC in order to be able to propose 
Coordination and gapping in Catalan Sign Language (LSC)

a syntactic analysis of coordination. While till now no account for adversative coordination has been proposed, we will adapt Vicente's (2010) analysis for English to LSC. The structure proposed for conjunction will be used also for disjunctive and adversative coordination. Moreover, it aims to be extended to other SLs and to cover also the data presented for spoken languages in 2.2. 


\section{COORDINATION IN CATALAN SIGN LANGUAGE (LSC)}

This chapter aims to characterize coordination in Catalan Sign Language (LSC) by providing a description of how it is expressed and a syntactic analysis for it. In 3.1 we will see how coordination is marked in LSC, describing the different types of coordination and how they are expressed. In 3.2 we will focus on the properties of conjunction mainly, and will also go through the tests to identify coordination in LSC. We will then look, in 3.3, at the syntactic analysis proposed for LSC in order to account for the data presented in 3.1 and 3.2. Moreover, we will extend it also to disjunctive and adversative coordination. In 3.4, we will provide cross-linguistic data to support the analysis for coordination in LSC showing that it can also be applied to other SLs. The directionality of this structure will be further supported by data on gapping that will be presented in 5.6.

This chapter will provide data mainly on TP coordination, but I assume that the same strategies and analysis can also be applied to NP coordination.

\subsection{Expression of coordination in LSC}

In LSC, as in spoken languages and other SLs, there are three types of coordination: conjunction, disjunction and adversative coordination.

As presented in 2.3.2, in SLs it is possible to express coordination syndetically or asyndetically depending on the presence 
of a manual marker linking the conjuncts or only with NMMs. In LSC, as in other SLs, conjunction and disjunction are predominantly expressed asyndetically using juxtaposition through NMMs. Adversative coordination, instead, tends to use the coordinator BUT between the two conjuncts. In (116) we can see an example for each type. In (116a) conjunction is expressed using juxtaposition marked with body shift towards the ipsilateral side spreading on the second conjunct to mark it; on the first one no clear NMMs are used due to the articulation of the two signs on the body: MARINA is signed anchored on the face and DANCE involves a specific movement of the whole body. Additional NMMs are then impossible to produce. Disjunction in (116b) also shows juxtaposition and the two conjuncts are marked mainly with head lean towards opposite sides of the space. Moreover, important NMMs are the mouthing $[o]$ meaning "or" produced between the two conjuncts and the facial expression conveying uncertainty or doubt, which is characterized by furrowed eyebrows and squinted eyes. In (116c), adversative coordination displays the manual marker BUT between the conjuncts, which are also put in contrast thank to body leaning forward on the second conjunct.

(116) a. MARINA DANCE JORDI CHAT. (11.20)

'Marina danced and Jordi chatted.'
fe+sq
hl+sp. ipsil. [or] hl+bbl contr.
b. MARINA WORK
VACATION. (16.02)
'Marina will work or she'll go on vacation.' 
re

bl forward

c. JORDI VERY-TALL BUT BASKETBALL PLAY VERY-BAD. (78.07)

'Jordi is very tall but he's very bad at playing basketball.'

In the next subsections we will see in detail how these three types of coordination are expressed. In 3.1.1 we will present data on conjunctive coordination, in 3.1 .2 on disjunctive and in 3.1 .3 on adversative.

\subsubsection{Conjunctive coordination}

In this section we will look at the strategies used to express conjunction in LSC, and present the manual and non-manual markers used. As introduced above, conjunction is mainly asyndetic, expressed through juxtaposition. We will first focus on the NMMs used to express it and we will then describe the manual markers that can accompany the NMMs just presented.

\subsubsection{NMMs for conjunctive coordination}

The NMMs used to mark the two conjuncts in conjunction are body or head shift or lean directed to opposite sides of the signing space for each conjunct. In the case of body shift, the torso of the signer rotates towards the contralateral or the ipsilateral side of the space. The same can happen with the head that will rotate towards one side or the other. In the case of body or head lean, the torso or the head bend towards one side of the space or the other, without rotating. 
These NMMs are often used in combination with the localization in space of at least one element in each conjunct. The signs that are realized in the space are placed in either side of it, without preference for the direction selected for the first and the second conjunct. When more than two conjuncts are expressed, they tend to alternate from one side to the other. The signs that are body anchored, instead, are marked with body or head shift or lean. In (117a) we can see an example of TP coordination ${ }^{12}$ where the two conjuncts are marked with body shift towards opposite sides of the space. The signs in the first conjunct are all body anchored, while in the second one BUY is signed in the ipsilateral side of the signing space. In (117b), repeated from (116a), the first conjunct is body anchored and no shifting or leaning is expressed due to the characteristics of DANCE, which involves the whole body in its lexical form. It is important to notice that the first part of the sign is directed towards the contralateral side, though. In the second conjunct, instead, both the subject and the verb are produced in the ipsilateral side of the space, the same side the body is rotating to. We can see then that the NMMs tend to spread over the whole conjunct, but when they are constrained, other cues like the use of the space can compensate for their absence.

\footnotetext{
12 As we will see in chapter 4, for verb agreement I will adopt the minimalist approach proposed by Costello (2016) and Pfau et al. (2018) in which the head of $\mathrm{TP}$ is always active. Either it is filled through $\mathrm{V}$ to $\mathrm{T}$ movement or through its activation due to the presence of NMMs or an auxiliary that compensate for the lack of overt agreement in space. For more information about the proposals by Costello (2016) and Pfau et al. (2018), see 4.1.1.
} 


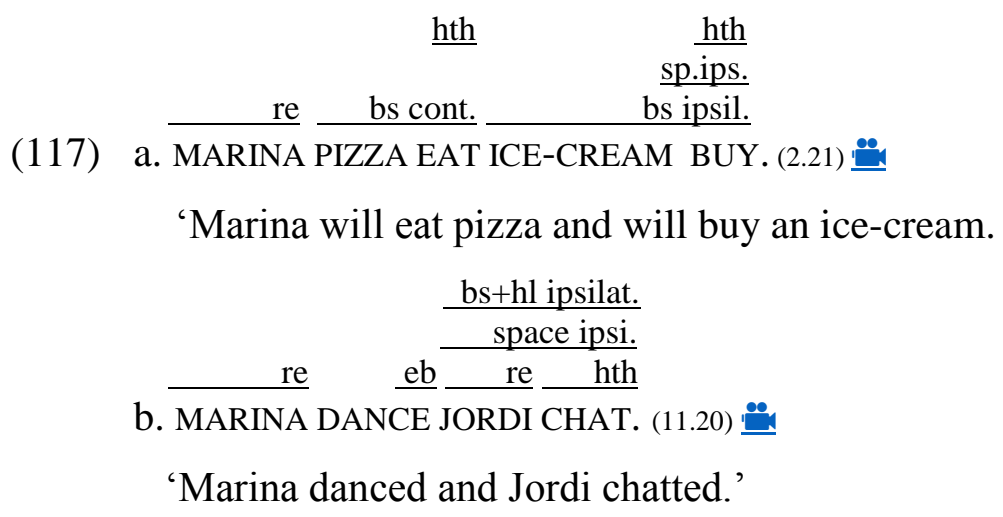

In (117a) the use of head thrust on the last sign of each conjunct should be noticed. In (117b), it is also possible to see the use of eye blinking at the end of the first conjunct. In other examples, these NMMs can be substituted by a single head nod, as in HKSL (Tang and Lau 2012) or by a slightly forward movement of the head, very close to a head thrust. Göksel \& Kelepir (2016) point out that in TiD head thrust is a marker of TP boundaries and authors like Wilbur (1994), Herrmann (2010) and Pfau \& Quer (2010) identify eye blinking as a clause and constituent boundary marker. The data in (118) show that also in LSC these NMMs are boundary markers, even though head thrust is also found marking a TP domain. The presence of these NMMs also shows a cross-linguistic parallel between the SLs that have been studied for coordination.

As for the spreading of NMMs, it is important to underline that, in LSC, coordination can be detected from the beginning of the sentence, or at the latest starting from the second element of the sentence, especially in cases of TP coordination, if we put aside special cases like the one in (117b). This property is unique of SLs in 
comparison to spoken languages where coordination can be detected only later in the structure ${ }^{13}$.

In LSC, as in ASL (Padden 1988), HKSL (Tang \& Lau 2012) and Auslan (Johnston \& Schembri 2007), juxtaposition of TPs can be used to express simultaneous (118a) or sequential events (118b). In LSC, though, sequential events can be expressed via juxtaposition only if supported by pragmatics, as in (118b), otherwise it is necessary to use a time adverb like THEN or LIST-BUOY-की, which we will see in 3.1.1.2. The default interpretation of conjoined TPs, otherwise, is that of simultaneous events. In cases of TP coordination, like in $(117 \mathrm{c}, \mathrm{d})$, the interpretation depends on the context. In (118c), repeated from (117a), for example, in a context where somebody asks the interlocutor what Marina did when she went to the bar, the interpretation of the order of the conjuncts would be atemporal, providing general information. Out of the blue, interpreting it only on the base of pragmatics, instead, the conjuncts in (118c) would be in sequential order, as it also the case in (118d) where the two conjuncts cannot be inverted for pragmatic reasons.
sp. cont. bsths contr. space ipsi. bsths ipsi.
a. MARC MATH TEACH MARINA WRITE-ON-THE-BOARD. (14.06)

'Marc is teaching math and Marina is writing on the board.' bs contralat bs ipsilateral re hth re shn

b. MARINA CAKE MAKE JORDI SELL. (75.16) 'Marina made a cake and Jordi sold it.'

\footnotetext{
${ }^{13}$ I am grateful to Caroline Féry for pointing this out to me.
} 

$\underline{\text { hth }}$
$\underline{\text { hth }}$
sp.ips.
re bs cont.
bs ipsil.
c. MARINA PIZZA EAT ICE-CREAM BUY. (2.21)
'Marina will eat pizza and will buy an ice-cream.'
space cont. bs+space ipsi. re hl+bs contr. hl contr.
PILE. (79.02)
d. MARINA SANDWICHES MAKE
'Marina made some sandwiches and piled them.'

Having a specific context, also a simultaneous reading could be accommodated. Another strategy to express two simultaneous events consists in taking advantage of simultaneity in the visual-gestural modality: it is possible to use both hands at the same time with the left one expressing one event and the right one expressing the other one. We can see an example in (119a) where the verb WALK is first introduced alone and then the dominant hand keeps signing WALK while the dominant one produces a one-handed version of the verb EAT expressed as a verbal classifier. This type of strategy, in which both hands keep their movement, is not as common, though, due to phonological and articulatory limitations in the production of the signs. Kimmelman (2017) argues that this type of structure is never produced, but LSC seems to be an exception. It is true, though, that it is more common also in LSC to find the non-dominant hand holding one of the signs, while the dominant one produces the other, like in (119b). In (119b) we can see that the sign CL:STIR keeps its movement till the end of the sentence, while WATCH, after being produced, gets held. The same can be found with classifiers 
constructions (CL) as in (119c), where the classifiers for driving a car and a truck are used simultaneously in the first part of the example. In the second part, instead, the production of the classifier for PULLOUT is first used for the car and then for the truck, expressing sequential events. Simultaneous use of both hands is not unique to LSC, it also found in FinSL (Jantunen 2016) and HKSL (Tang \& Lau 2012), as we saw in 2.3.2.1.

$$
\text { bl+space ipsil. } \quad \text { alternation head shift+eye gaze }
$$

a. WALK $\quad \mathrm{hd}_{\mathrm{dom} .}$ : WALK------

$$
\begin{aligned}
& \text { hs contr. } \\
& \text { hd }_{\text {non-dom. }} \text { CL:EAT---- }
\end{aligned}
$$

'I was walking and eating (looking around)'.

b. MARINA COOK CL:COOK---hddom.: $\frac{\mathrm{hl} \mathrm{ipsi.}}{\mathrm{CL}: \mathrm{STIR}(80.13)}$

$$
\mathrm{hd}_{\text {non-dom. }} \text { : } \frac{\text { space+eg contralateral }}{\text { WATCH--------------- }}
$$

'Marina was cooking and watching (something).'

c. GROUND WET RAIN-A-LOT CAR

space contr.

( hd $_{\text {dom: }}$ CL $\left._{c a r}\right)$ TRUCK CL:DRIVEcar space ipsilatereal

hd $d_{\text {non-dom. }}$ : CLtruck CL:DRIVEtruck CL:SLIDE CL:HIT space contr. PEOPLE COME PULL-OUT hd $_{\text {dom: }}$ CL:PULL-OUT car $_{\text {r }}$

$$
\text { hd }_{\text {non-dom. }} \frac{\text { space ipsilatereal }}{\text { CL:PULL-OUT }}
$$

'The ground was wet, it rained a lot. A car and a truck were driving and the truck started sliding and it hit the car. People came to pull them out. They pull the car out and then the truck.' 
The examples above are a sample of how conjunction is produced in LSC. It is possible to confirm that conjunction in LSC is expressed asyndetically, because only the use of NMMs is obligatory. Manual markers, instead, as we will see in 3.1.1.2, are always optional and in the sentences in which they are used, the NMMs we just described are anyway present.

We will look next at the manual markers used in conjunction in LSC.

\subsubsection{Manual markers for conjunction}

In LSC, the manual markers found in conjunction are the following and each one will be described in detail. As we will see, only ALSO and PLUS will be considered coordinators.

i) List-buoys

ii) $\quad$ ALSO

iii) PLUS

iv) THE-2

v) $\mathrm{BOTH}$

List buoys in LSC shows the same characteristics we saw for the other SLs in 2.3.2.1. This strategy is mainly used in coordination for listing, but it can also be used to express temporal order between the conjuncts. In LSC, list buoys can express a range between 2 and 5 or 10 referents, depending on the preference of the signer. Till 5, the number is always held on the non-dominant hand. The dominant one, instead, points to the fingers on the non-dominant hand, and can have 
different handshapes, used for different purposes: the - -handshape with a straight movement to the fingers of the non-dominant hand (Figure 1) neutrally refers to an individual or a proposition containing an event, but if used with an arc movement and puffing of the chicks (Figure 2), it places the individuals or propositions in a temporal ordering. The same meaning is expressed using the combination with puffed cheeks and an arc movement towards the fingers of the non-dominant hand (Figure 3). Instead of thandshape, it is also possible to find 7 -handshape, but productive. The expression of temporal order with का or 5 -handshape is related to the sign meaning "turn" that is expressed with the same handshapes but it is usually produced in the space starting from a referent and directed to another one in order to convey "passing the turn to somebody else" or to simply indicating that it is the turn of another person. List-buoys and the sign for "turn" are then integrated together. Moreover, an important property of list buoys consists in having the non-dominant hand that marks the number of the referents held still during the production of the conjuncts. It is not necessary, and it does not need to be held during the whole sentence, but it is a common feature due to the simultaneous nature of SLs. Vermeerbergen, Leeson \& Crasborn (2007) refer to this phenomenon as "weak hand hold" but considerable attention to the properties of simultaneity in the literature of SLs have been given by several authors. 


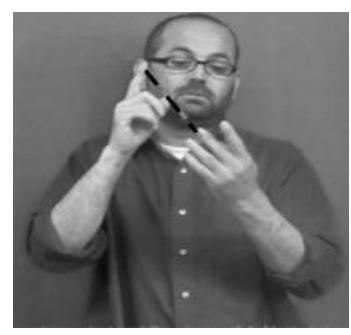

Figure 1: LIST-BUOYS-INDEX

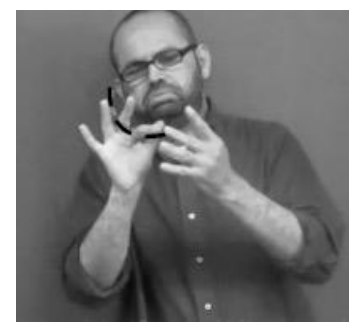

Figure 3: LIST-BUOYS- (puffing)

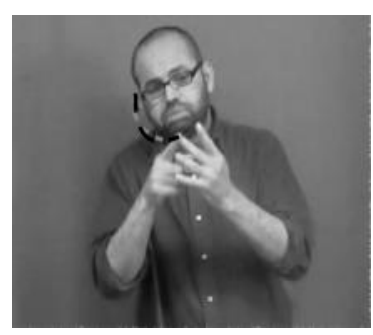

Figure 2: LIST-BUOYS-INDEX (puffing)

In (120), we can see some examples where a list-buoy with handshape is used. In the gloss, LIST is followed by a number that corresponds to the amount of the total referents and afterwards it is marked the finger that the index touches on the non-dominant hand ${ }^{14}$. When having four referents, the first one is placed on the index finger, while with five, the listing starts on the thumb. Despite the use of an overt marker, the typical NMMs for conjunction are used. In (120) we can see some examples of list-buoys. In (120a) the non-dominant hand is not held through the whole sentence but it is the case in (120b, c) with the realization of "weak hand hold". There is also another way to refer to the referents in the conjuncts, but it is just a listing strategy

\footnotetext{
${ }^{14}$ I am using the same glosses adopted by Kimmelman (2017) also because I will refer back to his analysis when presenting the one for coordination in LSC (see 3.3.1.2).
} 
and does not involve list-buoys per se, like in (120c). As we can see, the total number of referents is not present in both conjuncts and the pointing to the fingers is used just as a list.

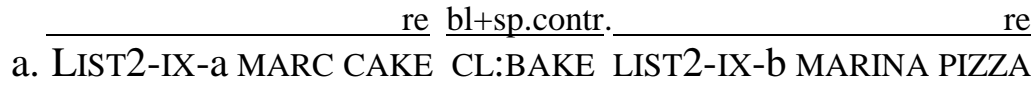
bl+sp. ips.

CL:BAKE. (1.17)

'On the one hand, Marc baked a cake and on the other, Marina baked a pizza.' hl contralateral re sp. contr.

b. hddom: (LIST2)-IX-a MARINA PIZZA CL:BAKE

hl ipsilateral

re sp. ipsi.

(LIST2)-IX-b JORDI CAKE CL:BAKE

hd non-dom: LIST2 $_{\text {- }}$ (80.08)

'On the one hand, Marina baked a pizza and on the other, Jordi baked a cake.'

c. hddom: YESTERDAY (LIST3)-IX-a,b,c (LIST3)-IX-a IX-1

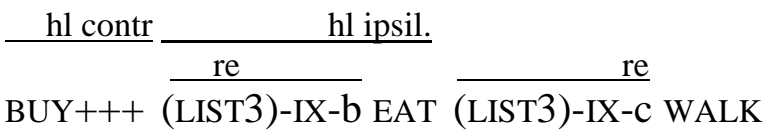

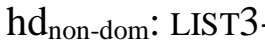
(80.09)

'Yesterday (I did) three things: I went for shopping, I ate, I walked.'

hl+bl+space ipsil.

re re

d. LIST1-IX-a MARINA CAKE CL:BAKE LIST2-IX-b JORDI PIZZA sp.ipsi.

CL:BAKE. (81.08)

'Marina baked a bake and Jordi baked a pizza.' 
As for the position where this marker can be placed in the sentence, list-buoys can be found either at the beginning or at the end of each conjunct, as we can see in example (120), where LIST1 and LIST2 precede the conjuncts, or as in (121), where they follow them.

$\frac{\text { re }}{\text { MARC CAKE CL:BAKE. }} \frac{\text { shn }}{n} \frac{\text { bs+space ipsil. }}{\text { re }}$
$\frac{\text { Lhn }}{\text { LIST2-IX-b. (1.18) }}$
'Marc baked a cake, on the one hand, and Marina baked a
pizza, on the other.'

A second manual marker is $A L S O$, which, together with another one, PLUS, is the most similar coordinator to the English "and", mainly because of its position between the conjuncts. In TP coordination it can only be found introducing the conjuncts, as in (122), and we can see a representation of it in Figure 4.

hl ipisl. sp.cont. hl ips ipsi.

$\underline{\text { shn }} \underline{\underline{\text { hn }}}$

(122) MARC CAKE BAKE ALSO MARINA PIZZA EAT

hl $\quad \underline{\text { shn }}$

ALSO IX-2 SANDWICH PREPARE. (1.25)

'Marc baked a cake, Marina ate a pizza and you prepared a sandwich.' 


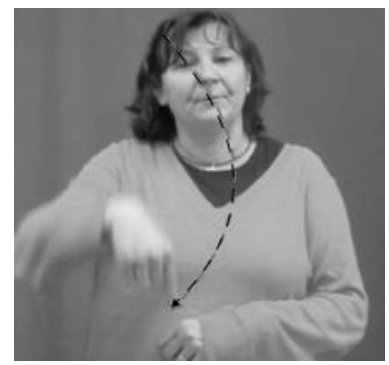

Figure 4: ALSO

Applying to LSC the prosodic grouping test (Haspelmath 2004) that we presented in 2.1.3, in (123) we can see that ALSO can be signed in a continuum with the second conjunct following a short prosodic break after the previous conjunct. The break is marked by a comma and we can see that the coordinator belongs to the second conjunct. As we have already seen, this position of the coordinator determines, then, the presence of prepositive coordination (Haspelmath 2004), where the coordinator belongs to the second conjunct, in opposition to postpositive coordination, where the coordinator belongs to the first one (see more in 3.2.2). This aspect will be crucial among the properties we will look at in 3.2 in order to determine the coordination structure for LSC, in particular in establishing its directionality, on the basis of Zhang's (2010) proposal.

hl ipisl. sp.cont.

$\underline{\text { shn }} \underline{\text { shn }}$

(123) MARC CAKE BAKE // ALSO MARINA PIZZA EAT // ALSO IX-2

hl ipsi. $\quad \underline{\text { shn }}$

SANDWICH PREPARE. (1.25)

'Marc baked a cake, Marina ate a pizza and you prepared a sandwich.' 
PLUS, which is also used to express "addition" to a list of referents, can be expressed with or or -handshape. In the sign with handshape, the wrist does an arc movement from the vertical to the horizontal plane, as in Figure 5, while with -handshape, the sign is oriented towards the vertical plane and moves upward with an arc movement, as in Figure 6. PLUS- Th mostly used by the female population while in the male one it is considered the old, almost extinguished, version of PLUS- Moreover, both signs can be produced with the mouthing más [mas] meaning "plus" in Spanish. In future glosses, we will distinguish between PLUS- and PLUS- की but we will refer to both using PLUS.

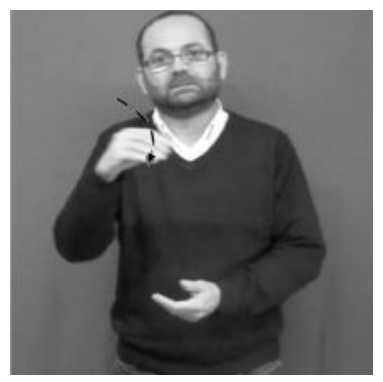

Figure 5: PLUS-

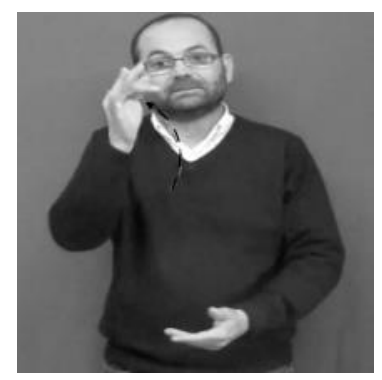

Figure 6: PLUS- की?

In (124) we see an example where PLUS- is produced between the conjuncts, the only position where it can appear, as we saw also for ALSO.

hl cont. bl+hl ipsi.

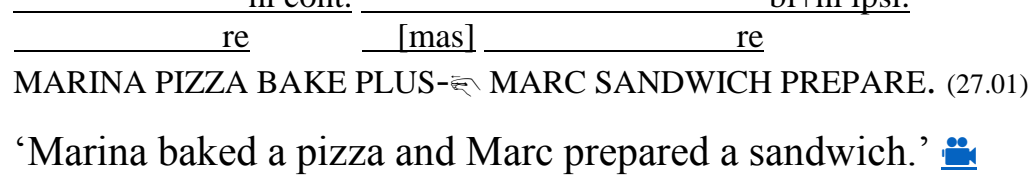


Both ALSO and PLUS, more than the other manual markers, are important to determine the position of the coordinator with respect to the conjuncts since they are signed between them. Moreover, as we can see in (125), they can be used in the same sentence when more than two conjuncts are produced ${ }^{15}$. PLUS- can only be used as the last coordinator while ALSO is used to coordinate the previous conjuncts. PLUS- - , when used in combination with ALSO, it has the function of putting emphasis on the last conjunct.

$\frac{\text { bs contralateral }}{\frac{\text { sp.con. }}{\text { (125) }}} \frac{\text { hl+bs ipsilarteral }}{\text { space ipsi. }} \frac{\text { re }}{\text { hl ipsilateral }}$
$\frac{\text { s.contr. }}{\text { SON SCHOOL BRING. (80.07) }}$
'Marina went home and she bought the ticket for the cinema
and she brought her son to school.'

Going back to ALSO, in TP or NP coordination, it has additive properties that it keeps when used as focus additive marker (see 5.1 when used in gapping). In coordination, ALSO presents the same characteristics as in languages where this type of adverbial connectors develops into conjunctive markers (Mithun 1988) and therefore it can have different functions depending on the sentence. In order to make sure that in coordination it is not the focus marker that is used, but ALSO as a coordinator, we can look at the following

\footnotetext{
${ }^{15}$ This aspect will be important in showing the need to distinguish the two syntactic phrases that host the two different coordinators in the syntactic representation, as we will see in 3.3 .
} 
data in (126). The focus marker is always used at the end of the second conjunct in elliptical structures, like the case of stripping in $(126 a)^{16}$. In coordination, instead, ALSO is always between the conjuncts, as in (126b). Trying to put ALSO before the remnant in (126a) will then result in a case of NP extraposition, like in (126c). The clear evidence for this is in (126d), where in a clear case of NP coordination it is not possible to have ALSO after the second conjunct.

a. MARINA ICE-CREAM PAY [CHOCOLATE ALSO].

'Marina paid for an ice-cream and for a chocolate, too.'

b. MARINA ICE-CREAM [ALSO CHOCOLATE] PAY. (73.09)

'Marina paid for an ice-cream and for a chocolate.'

c. MARINA ICE-CREAM PAy [ALSO CHOCOLATE]. (73.12)

'Marina paid for an ice-cream and for a chocolate.'

d. *MARINA ICE-CREAM [CHOCOLATE ALSO] PAY. (73.10)

From the examples in (126), it is evident, then, that ALSO can be used as a coordinator or as a focus additive marker depending on the type of sentence and on its position.

This sign can also play an important role in NP coordination. In (127b) we can see that ALSO makes sure that the signs JORDI and SON are recognized as two referents, removing the possibility of interpreting the two NPs as one, that is "Jordi's son", as in (127a). In

\footnotetext{
${ }^{16}$ Stripping is a type of ellipsis that is described by Hankamer and Sag (1976: 409) as "a rule that deletes everything in a clause under identity with corresponding parts of a preceding clause except for one constituent (and sometimes a clause initial adverb or negative)". We will look more in detail at this structure in LSC in 5.1.
} 
(127b), ALSO adds a distinct referent for JORDI without interpreting him as the possessor.

a. JORDIj SON IX-3j-POSS CINEMA GO.

'Jordi's son went to the cinema.'

b. JORDIj ALSO SON IX-3j-POSS CINEMA GO. (78.16) 를

'Jordi and his son went to the cinema.'

Similar properties in NP coordination are shown by the marker THE2 that can also play an important role in extending the reading of negation over both conjuncts. This marker is the sign for the English "both". More than a coordinator, it is a pronominal element that refers back to the two conjuncts previously introduced, whether they are NPs or TPs. In (128b) we can see that THE-2 makes sure that the NPs JORDI and SON are recognized as two referents, avoiding having the same interpretation of (127a) where Jordi is the possessor, repeated in (128a). In (129), we can see how THE-2 also affects negation. In (129a), the negation cannot scope over both conjuncts, but THE-2 refers back to the two conjuncts previously topicalized in order to make the negation take scope over both (129b).

(128) a. JORDIj SON IX- 3 j-POSS CINEMA GO.

'Jordi's son went to the cinema.'

b. JORDIj SON IX-3j-POSS THE-2 CINEMA GO. (78.15)

'Jordi and his son went to the cinema.'

a. *MARINA MEAT JORDI FISH EAT NOT. 


\section{b. MARINA MEAT JORDI FISH THE-2 EAT NOT. (76.02) 'Marina doesn't eat meat and Jordi fish.'}

We will not focus on this property of THE-2, but it is important to underline it, especially because it is peculiar to NP coordination, since we assume here that all the characteristics of TP coordination extend to it, too.

A similar sign to THE- 2 is glossed as same meaning but a different distribution in the sentence. In Figure 7 and 8 we can see the representation of these two signs. THE- 2 is characterized by the -handshape with the palm facing up and the movement going from one side of the space to the other where the two conjuncts were placed. THE- 2 is also often characterized by the mouthing dos [doz] in Spanish, 'two'. BOTH, instead, is a two-handed sign where both hands have -handshape and while the nondominant hand stays still, the dominant one taps the two selected fingers on top of the ones of the other hand. Also вотн can be produced using the mouthing for "two" in Spanish.

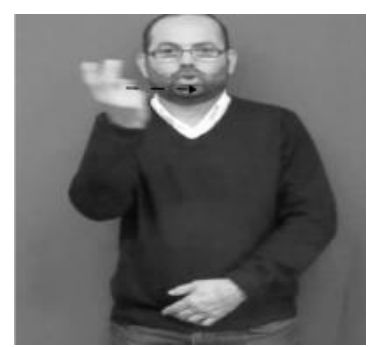

Figure 7: THE-2

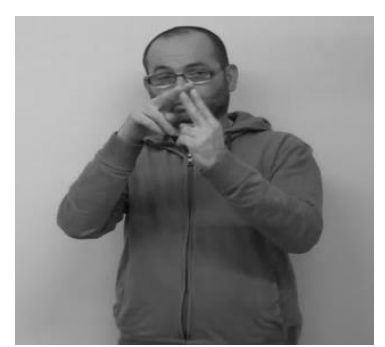

Figure 8: ВОTH 
Both signs are used to mark the accomplishment of the two events introduced in the previous propositions, as in (130).

(130) a. MARINA PIZZA EAT ICE-CREAM BUY THE-2. (2.25)

'Marina both ate pizza and bought ice-cream.'
b. MARINA PIZZA EAT ICE-CREAM BUY BOTH. (2.24)

'Marina both ate pizza and bought ice-cream.'

With respect to frequency, THE-2 is used more often than BOTH. This might be due to the fact that THE- 2 is also used as a second person dual pronoun. Both markers, even if used to support the coordination of two conjuncts, they are mainly referential elements that do not directly function as coordinators. As for the position of these markers, both THE-2 and BOTH can appear either at the beginning, as topicalized elements, as in (131), or at the end of the sentence, as in (130). In (131b) we can see that also вОтH can appear at the beginning of the sentence, even though THE- 2 is preferred and therefore more productive.

hl+bs contr. hl+bs ipsi.

a. THE-2 MARINA PIZZA PREPARE MARC ICE-CREAM MAKE. (20.06)

'Two things, Marina prepared pizza and Marc made icecream.' 


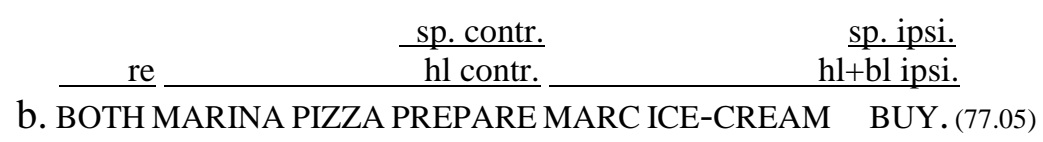
'Two things, Marina prepared pizza and Marc bought icecream.'

Let us look now at how disjunction is expressed in LSC. Also in LSC, as in languages like ASL (Davidson 2013), conjunction and disjunction show similar NMMs. Let us see, then, how they can be distinguished.

\subsubsection{Disjunctive coordination}

Disjunction in LSC, as pointed out for other languages like ASL (Davidson 2013), is mainly expressed asyndetically and it shows very similar NMMs to conjunction. In the next subsections we will see the non-manual and manual markers that contribute to make disjunction detectable in LSC.

\subsubsection{NMMs for disjunctive coordination}

Disjunction in LSC is expressed using body or head lean or shift to mark two or more disjoint NPs or TPs. Other NMMs such as squinted eyes, furrowed eyebrows and both lips curved down spreading over the constituents are necessary, mainly to express the epistemic status of the speaker or of a referent. Moreover, the mouthing [o] 'or' produced between the disjoint constituents is very productive. In 
Figures 9 and 10 we can see an example of these NMMs and an example of a full sentence is presented in (132).

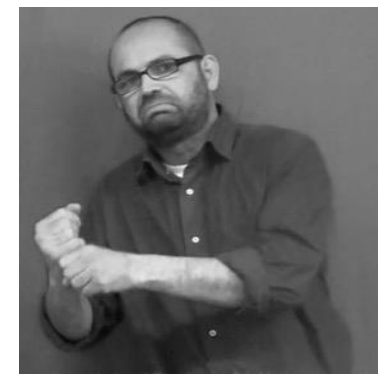

Figure 9: NMMs

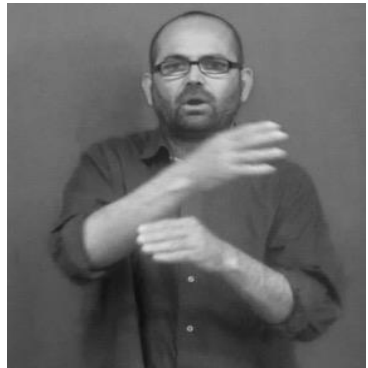

Figure 10: [o] mouthing for 'or'

$$
\begin{aligned}
& \text { fe+sq } \\
& \text { hl+ sp. ipsil. [o] hl+bbl contr. } \\
& \text { MARINA WORK VACATION. (16.02) }
\end{aligned}
$$

'Marina will work or she'll go on vacation.'

The NMMs expressing epistemicity are not all used at the same time, as we can see in (132), where the lips are not curved down. We will see the distribution of this NMMs in the examples in 3.1.1.2, where also manual markers will be present. Without any additional manual marker, as also pointed out by Davidson (2013) for ASL and reported in 2.3.2.2, the sentence in (132) is ambiguous between an inclusive and an exclusive reading. In order to make this distinction explicit, it is necessary to add one of the overt markers we will see next, namely EITHER-ONE, WHICH or THE-2alternate, for an exclusive reading. For an inclusive reading, instead, it is possible to add to an expression such as THE-TWO CAN, IX-1 DO NOT KNOW, meaning "can be both, I do not know". We can see an example of the first type in (133a) and of the second one in (133b). 
(133) a. MARINA PALM-UP HOUSE GO SCHOOL STAY EITHER-ONE. 'Marina either went home or stayed at school.' (2.04) $\mathrm{lcd}+\mathrm{fe}$

b. MARINA BOOK READ $\frac{\mathrm{hl}+\mathrm{bl} \text { contr. [o] }}{\mathrm{hl}+\mathrm{bl} \text { ipsi. }} \frac{\text { re }}{\text { TV WATCH, THE-TWO, KNOW-NOT. }}$ 'Marina read a book or watched tv, can be both, I don't know.' (83.07)

We will look next into the manual markers that help disjunctive coordination to be further detected in contrast to conjunction.

\subsubsection{Manual markers for disjunctive coordination}

In combination with NMMs it is often possible to see the production of some manual signs, that will be described in detail one by one. Other than OR, which is not productive, these signs are not disjunction markers per se, but elements that help expressing disjunction, marking epistemicity or identifying the disjoint NPs or TPs. Let us see the list of these markers:

i) $\mathrm{OR}$

ii) PALM-UP

iii) DOUBT

iv) EITHER

v) $\mathrm{WHICH}$

vi) THE-2alternate 
$O R$ is the only disjunctive marker that it is possible to find in LSC. It is rarely used and it always appears in combination with the mouthing [o] from the Spanish word $o$ meaning "or". This sign keeps the होhandshape from the word it parallels. In Figure 11 we can see a representation and in (134) an example where it is used.

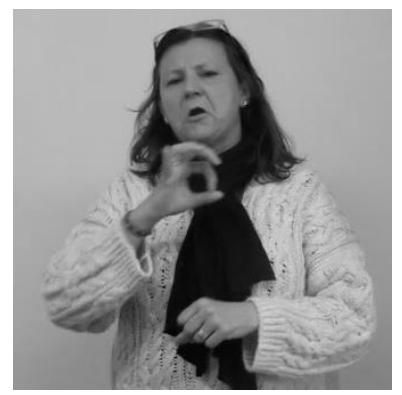

Figure 11: OR

(134) MARINA WORK $\frac{\underline{\mathrm{hl} \text { contr. }} \frac{\mathrm{o}]}{\mathrm{Ol} \text { ipsi. }}}{\text { VACATION. (16.04) }}$
'Marina will work or will go on vacation.'

The production of OR is very rare because it is often compensated by the mouthing [o] for "or" only.

The first sign used to help expressing disjunction in LSC that we will present here is PALM-UP. PALM-UP, signed with ${ }_{-3}^{-}$-handshape oriented upward, seems to be a prosodic marker that expresses doubt or uncertainty. It can be static or involve alternating movement. It is always found in combination with NMMs and eventually with others manual markers. In Figure 12 we can see a picture of the sign and in (135) an example where it appears. In (135), we can also see NMMs used such as body shift towards opposite sides of the space to mark 
the two disjoint TPs and both lips curved down spreading over them to express uncertainty.

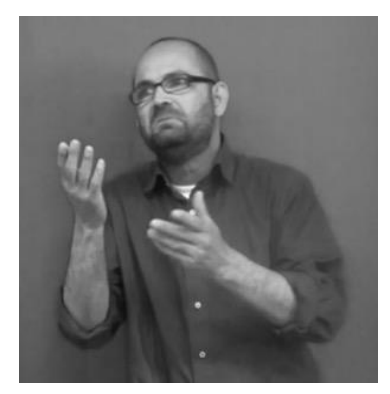

Figure 12: PALM-UP

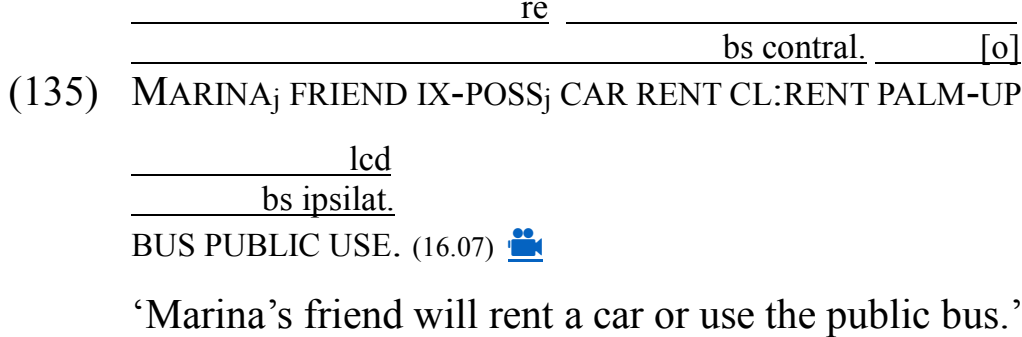

In (135), PALM-UP is produced between the two TPs but it can also be found in other positions: before each disjoint constituent (136a), at the beginning of both (136b) or at the end of the sentence (136c). As we can see in $(136 \mathrm{~b}, \mathrm{c})$, PALM-UP can be used in combination with other manual markers such as EITHER-ONE, which we will present in detail later in this subsection, and LIST-BUOYS, used to introduce to the two possible events they refer to.

lcd

bs+bl cont. bs+bl ipsi.

(136) a. MARINA PALM-UP HOME GO PALM-UP SCHOOL STAY. 'Marina doesn't know whether to go home or stay at school.' (2.01) 'بe 
b. MARINA PALM-UP HOUSE GO $\frac{\text { bl contr. }[\mathrm{o}]}{\text { SCHOOL STAY EITHER-ONE. }}$ (2.05)

'Marina either went home or stayed at school.'

c. THINK LIST1-IX-a MARINA CAKE BAKE LIST2-IX-b MARC

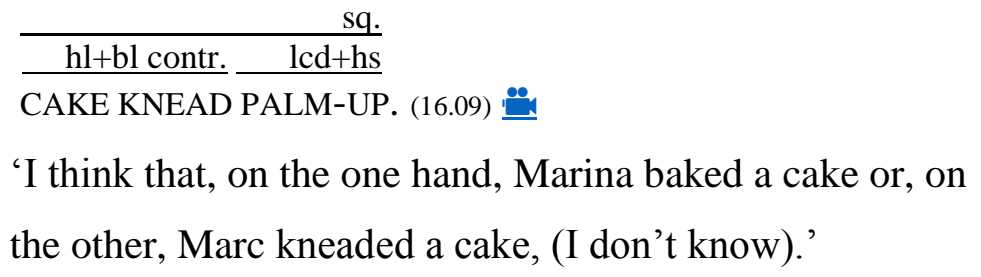

The different positions of PALM-UP in the sentence do not affect the meaning of the sentence.

Another sign used in disjunction is DOUBT, which helps to express the epistemic status of a referent in the sentence, depending on its position. We can see a representation of it in Figure 13, where the sign is produced in combination with NMMs typical of disjunction.

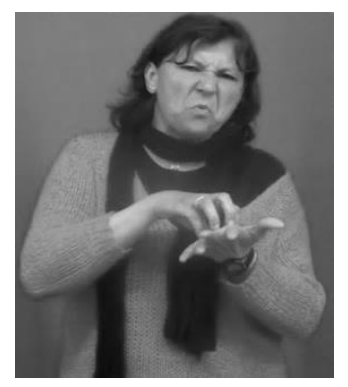

Figure 13: DOUBT. 
In the examples that follow in (137), (138) and (139) we can see how the different positions of DOUBT in the sentence can affect the attribution of epistemicity to the speaker or to another referent. DOUBT is often used also to introduce role shift (RS). Let us see its distribution:

i) when it is at the beginning of the sentence: reference to the knowledge of the speaker (137);

bl ipsi. sp. contr. [o]

(137) DOUBT LIST-1 MARINA PASTA KNEAD LIST-2 MARC CAKE

lcd

bl ipsi.

PREPARE. (16.30)

'I have a doubt whether, on the one hand, Marina kneaded pasta or, on the other, Marc prepared a cake.'

ii) when it is after a referent: reference to the knowledge of the referent it follows (138a), also when used inside role shift, as in $(138 b)$;
lcd
bs+space cont. $[0]$ bs + space ipsi.
a. MARINA DOUBT LIST-1 PIZZA KNEAD LIST-2 CAKE PREPARE.

(16.29)

'Marina doesn't know if, on the one hand, she'll knead a pizza or, on the other, if she'll prepare a cake.'

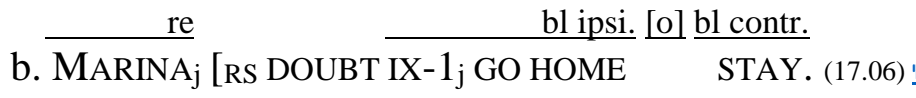

'Marina had a doubt "I go home or I stay".' 
iii) when it is at the end of the two constituents: it is ambiguous. Depending on the context and on the NMMs, it can refer to the knowledge of the referent or of the speaker, as in (139a). Inside role shift, though, there is no ambiguity, it can only refer to the knowledge of the referent introduced outside of role shift, as in (139b).

bs contr. bs ipsi. $1 \mathrm{~cd}+\mathrm{fe}$

a. MARINA PIZZA EAT ICE-CREAM BUY DOUBT. (2.26)

'I don't know if Marina ate pizza or bought ice-cream.'

b. MARINA $A_{j}\left[R S\right.$ IX- $1_{j} \frac{\text { bl contr. }}{\text { HOME STAY GO DOUBT]. (17.10) }}$ 'Marina (thought) "I stay home or I go, I don't know".'

The syntactic category to attribute to DOUBT is either of predicate or noun. It can appear modified by frequency morpheme, as in (140a) or by a negative sign used only to modify nouns, that is THERE-BENOT meaning "there is not", as in (140b), or by a numeral and an adjective, as in (140c). In the first case it clearly appears as a verb while in the other two as a noun.

$\mathrm{fe}+\mathrm{sq}$
hl ipsi.

BAKE MARC ICE-CREAM BUY. (22.03)

'Every weekend I always doubt whether Marina will bake the pizza or Marc will buy the ice-cream.'

(Context: the speaker is the owner of a restaurant and every weekend she has the same doubt). 
hl+bl ipsi. hl+bl cont.

b. HOME STAY SCHOOL GO [RS MARINAj IX-1 $1_{j}$ DOUBT

THERE-BE-NOT SCHOOL GO SURE]. (19.01)

'Whether stay at home or go to school, Marina "I don't

have any doubt, I'll go to school for sure.",

lbd+sq

bl cont. bl ipsi.

c. IX-1 DOUBT STRONG HOME STAY LEAVE. (23.20)

'I have a strong doubt, whether to stay at home or to leave.'

The grammatical category of this sign is not relevant for its function in disjunctive coordination since it is mainly an element that helps to express more clearly the epistemic knowledge of the referents in the sentence. It is not obligatory, and it needs to appear in combination with the specific NMMs of disjunction.

The fourth sign we are going to look at is EITHER-ONE, which can be used in affirmative and interrogative sentences. It is a bimanual sign with -handshape. While the non-dominant hand does not move, the selected fingers of the dominant one move alternatively touching the tips of the selected fingers of the non-dominant hand, as we can see in Figure 14. It can also be characterized by the mouthing cuál [kwal], 'which'.

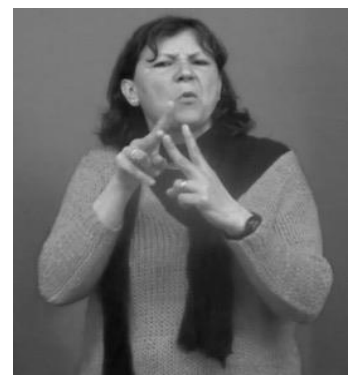

Figure 14: EITHER-ONE 
EITHER-ONE can be produced at the beginning or at the end of the two disjoint constituents, as in (141), where EITHER-ONE is also used inside role shift and in questions. In the latter case, EITHER-ONE can be repeated at the beginning and at the end of the two disjoint constituents, as in (141c). The different positions of EITHER-ONE do not affect the meaning of the sentence.
re

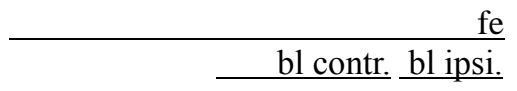

a. MARINA [RS EITHER-ONE HOME STAY GO?] (17.16)

'Marina (thought) "Either I/you stay at home or do I/you leave?"”

b. MARINA PALM-UP HOUSE GO $\frac{\mathrm{bl} \mathrm{contr} \text { [o] }}{\text { SCHOOL STAY }} \frac{\mathrm{bl} \text { ipsi. }}{\text { EITHER-ONE. }}$

'Marina went home or stayed at school, which one.'(2.05) fe

c. MARINA [RS EITHER-ONE HOME STAY GO EITHER-ONE?](17.01)

'Marina (thought) "Do I/you stay at home or do I/you leave?"”

There is another sign with similar properties to EITHER-ONE, even if it has a different distribution because it is generally produced only at the end of the sentence. This sign is glossed as WHICH and it is produced with an alternating movement of the two hands up and down. It is used to refer back to the two constituents introduced before and put them in competition forcing an exclusive reading of the sentence. We can see a picture of this sign in Figure 15. 


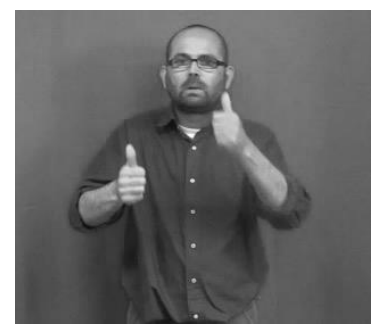

Figure 15: WHICH

WHICH is always used at the end of the sentence, as in (142). If it is found to introduce the disjoint constituents, they need to be under role shift, as in (142b).

(142) a. MARINA PALM-UP HOUSE GO SCHOOL STAY WHICH. (2.04)

'Marina either went home or stayed at school.'

bs ipsil. bs contr.

b. MARINA [RS WHICH SCHOOL STAY HOME GO]. (77.06) 'Marina (thought) "I stay at home or I go to school".'

Finally, another sign used in disjunction in LSC is THE2alternate. It is very similar to the sign THE-2 used in conjunction, but it has a different movement: the wrist rotates going from one side of the space to the other where the disjoint constituents where previously placed. We can see a representation in Figure 16. 


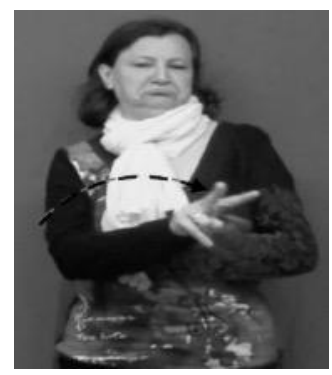

Figure 16: THE-2alternate.

It is preferably used at the end of the sentence, as in (143a), but it can also introduce the two disjoint constituents, as in (143b). It is never placed at the beginning of the sentence, though, because it would make the sentence weird, since it would have under its scope also the subject of the sentence.

(143) a. MARINA PIZZA KNEAD ICE-CREAM BUY THE-2ALTERNATE. 'Marina kneaded pizza or bought ice-cream.' (28.01)

b. $\frac{\text { re }}{\text { MARINA THE-2ALTERNATE PIZZA KNEAD }} \frac{\text { bs ipsi. }}{[0]} \frac{\text { bs contr. }}{\text { ICE-CREAM BUY. }}$ 'Marina kneaded pizza or bought ice-cream.' (28.02) 를

Looking at the manual markers that appear in disjunction in LSC shows that this type of structure is expressed asyndetically and that the signs produced support the NMMs, without being actual markers of disjunction. 


\subsubsection{Adversative coordination}

Adversative coordination in LSC, differently from conjunction and disjunction, is expressed mainly syndetically using the sign BUT in combination with the typical NMMs of coordination such as body or head shift or lean and use of space towards opposite sides of the space. We can see a picture of BUT in Figure 17.

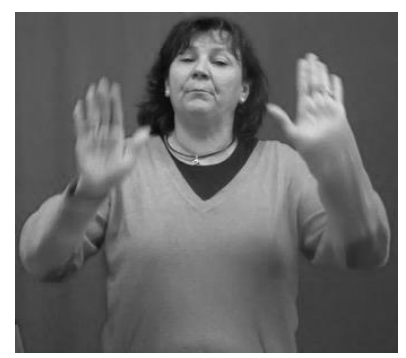

Figure 17: BUT.

BUT is usually produced between the two conjuncts to put them in contrast, as in (145a); however, depending on the type of adversative coordination, it might not be necessary.

The two main types of adversative coordination identified by Vicente (2010) in spoken languages can also be found in LSC. In (144a) we have counterexpectational contrast where two states of affairs are compared and the contrast is generated by the negation of the expectation provided by the context; in $(144 \mathrm{~b}, \mathrm{c})$ the contrast is corrective, through the denial of the proposition in the first conjunct, as we saw for spoken languages in 2.2.5.2. It is possible to notice that, in LSC, this type of adversative coordination can be expressed asyndetically. In counterexpectational adversative coordination, 
instead, if the sign BUT gets omitted, the meaning gets affected. Without the use of BUT, the expectation provided by the context is not negated in the second conjunct and the structure ends in a sequence of two statements, as we can see in (144a') for (144a). This is not the case for corrective adversative coordination.

re

bl forward

(144) a. JORDI VERY-TALL BUT BASKETBALL PLAY VERY-BAD. (78.07)

'Jordi is very tall but he's very bad at playing basketball.'

$\mathrm{fe} \underset{\text { re }}{\text { bl forward }}$

a'. JORDI VERY-TALL. BASKETBALL PLAY VERY-BAD. (78.06)

'Jordi is very tall. He's very bad at playing basketball.' space contr. re hs space ipsil. bl ipsil.

b. MARINA ROOM STUDY NOT IX-LOC GARDEN PLAY. (78.05)

'Marina is not studying in her room, she's playing in the garden.'

hl ips. hl ips.

re re

c. MARINA APPLE THREE EAT BUT BANANA ONE. (81.02) 'Marina ate three apples but one banana.'

Another strategy to express adversative coordination consists in using contrastive but marking overtly the contrast in polarity between the two conjuncts. We can see an example from Dutch, from Repp (2009), where the contrastive but is used in gapping. In this elliptical structure, that we will present in detail in chapters 4 and 5, the same verb used in the first conjunct is missing in the second one. As we can see in (145), in the first conjunct we can find the negation niet and in the second one the positive particle wel. In LSC we can find a 
similar structure, in gapping, as the one in (146a), and in a nonelliptical structure, as in (146b). The sign for YES is put at the end of the second conjunct while in the first one negation is expressed.

(145) Karel heft mijn kat niet genomen maar Harry

(Dutch) Karel has my cat not taken but Harry mijn hamster wel. my hamster PART 'Karen did not take my cat but Harry did take my hamster.'

(Repp 2009: 99)

(146) a. JORDI BEER CL:DRINK NOT BUT SANGRIA YES. (LSC) 'Jordi didn’t drink beer but sangria.' (81.04) 'ئ日e bs contr. $\mathrm{sq}+\mathrm{fe}$ bs ipsi.

b. MARINA PIZZA EAT DOUBT ICE-CREAM BUY YES. (3.34) 'Marina doesn't know if she'll eat pizza but she will buy ice-cream.'

In section 3.2.2, looking at the different properties of coordination in LSC, we will see in more detail the different types of contrastive structures expressed in adversative coordination and in 3.3.3 we will give an analysis for this structure in LSC.

Let us now present the syntactic properties of coordination in LSC in comparison with other SLs and spoken languages in order to have the basis to provide a syntactic analysis for coordination in LSC that can be extended to other languages as well. 


\subsection{Properties of coordination in LSC}

In sections 2.1 and 2.2 we looked at the properties that brought us to understand the different analyses for coordination proposed till now in the literature of spoken languages. In this section we will present the crucial syntactic properties of coordination in LSC, in particular conjunction, that will lead us to shape a syntactic analysis of this structure in LSC. The data presented will be mainly TP coordination, but the analysis can be extended to NP coordination, too. Moreover, due to the similarities between SLs in the strategies to express coordination, it will be possible to extend the analysis for LSC also to other SLs.

We will now look into the following characteristics of coordination in LSC: what categories can be coordinated in 3.2.1, the property of asymmetry between conjuncts in 3.2.2 and the tests used to detect coordination in LSC in contrast to subordination in 3.2.3.

\subsubsection{What can you coordinate?}

In Haspelmath's (2007) definition of coordination, he assumes that only categories of the same type can be coordinated. As Sag et al. (1985) and Munn (1993), among others, noticed for spoken languages, this definition does not hold, which argues against the Coordination of likes constraint (CLC) by Chomsky (1957) and Williams' (1978) Law of coordination of likes (LCL) that we presented in 2.2.2. In LSC, the CLC and the LCL do not apply either. As we can see in (147), different categories can be coordinated. In 
(147a) we have two TPs conjoined, in (147b) an AP and a TP and in (147c) an NP and an AP, in both cases as a complement to a covert copula $^{17}$. In (147d), the verb REMEMBER takes as complements an NP and a CP; the use of the overt marker ALSO helps to mark the two conjuncts. While the object of a verb is usually found in preverbal position in LSC, in (147c) we can see that both conjuncts are placed after the verb. This might be due to the heaviness of the two conjuncts together, as it happens in cases of heavy NP shift (Ross 1967), where a NP is found replaced in a position to the right of its canonical one, but also with sentential complements.

a. [TP MARINA DANCE] [TP JORDI CHAT]. (11.20)

'Marina danced and Jordi chatted.'

b. JORDI [AP IMPOLITE] [TP STUDY A-LITTLE]. (78.02)

'Jordi is impolite and he studies too little.'

c. JORDI [NP DOCTOR ALREADY OFFICIAL] ALSO [AP BUSY].

(78.18)

'Jordi is already officially a doctor and busy.'

d. MARINA REMEMBER [NP APPOINTMENT TODAY] ALSO [TP

HOUR GO PUNCTUAL IMPORTANT]. (52.15)

'Marina remembered today's appointment and that it's important that she goes there on time.'

\footnotetext{
17 (147b, c) could also be cases of TP coordination with a null subject in the second conjunct. There is not much literature on copular constructions in LSC so I consider the possibility of TP coordination for these two examples without taking any strong position on it.
} 
In the examples in (147) we can see that, in LSC, both conjuncts need to be licensed by the verb, therefore it is not possible to have cases like in the English (148) in which only the first conjunct has the right category needed to saturate the verb, while the second does not. In (148) we can see the known example from Sag et al. (1985) where the prepositional verb can only license noun categories. In LSC, the absence of prepositions makes the comparison to this type of structures impossible.

(148) You can depend on [NP my assistant] and [CP that he will be on time].

Zhang (2010), from examples like (148), gets to the generalization, which we saw in 2.2.2, that when different categories are coordinated, the one of the first conjunct is always the one that can saturate the verb while it is not the case for the category of the second one. She states that the first conjunct "must satisfy the category requirements that are imposed on the whole coordinate complex", as in (148). Moreover, she uses this generalization to assign to the whole coordination structure the category of the first conjunct. In LSC, though, this does not hold. The category of each conjunct, even when different, needs to saturate the verb. Therefore, the first conjunct shows the same status as the other(s) conjunct(s). In LSC, then, there is no reason to have a $\mathrm{X}$ category for the whole structure since there is no constraint for its selection like in English, which shows a preference for the category of the first conjunct. 
In the next section we will introduce more data on coordination in LSC, in particular about syntactic and semantic asymmetry.

\subsubsection{Asymmetries in coordination}

As we saw in 2.1.4 and 2.2, in coordination it is possible to talk about symmetry and asymmetry in the syntactic structure in order to establish whether the structure is flat or hierarchical. In LSC we can also observe such properties, as we will determine in this section. We will first introduce the semantic properties related to symmetry and asymmetry and then we will present the syntactic ones, to which more will be added going through the tests to identify coordination in contrast to subordination in LSC in 3.2.3.

\subsubsection{Semantic asymmetry}

Semantically, it is possible to identify symmetry and asymmetry looking at several aspects: the temporal order between conjuncts, following Nonato (2014), the presence of contrast (Mauri 2008; Hartmann 2015), and the instantiation of semantic subordination ( $\mathrm{Na}$ \& Huck 1992; Schmerling 1975; among others).

Based on Nonato's (2014) distinction we saw in 2.1.4, two conjuncts are symmetric when they can be inverted without affecting the meaning of the sentence. Coordination of simultaneous events respects this definition, while there is asymmetric coordination if the events are in temporal order. In 3.1.1 we presented coordination of 
events that are simultaneous and in temporal order. We can see in (149a, b) an example of simultaneous TPs. In (149c), two simultaneous TPs are produced using simultaneity of the two hands. The structures in (149) are considered to be semantically symmetric since the conjuncts can be inverted without affecting the meaning of the sentence. This does not apply to (149c) and (149d) due to a phonological restriction. The two conjuncts, in fact, are produced at the same time through the activation of both hands.

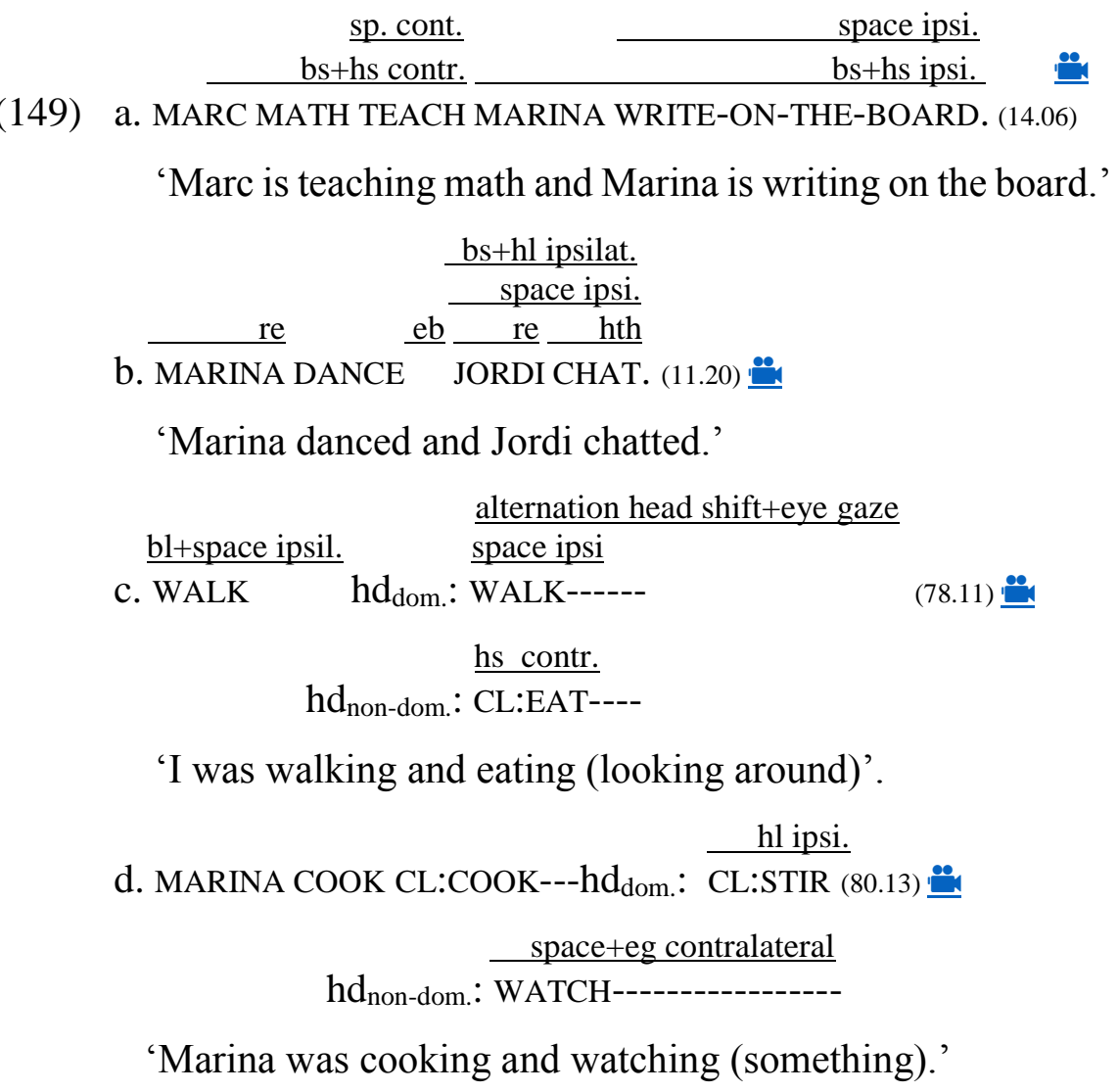

For the cases in which the temporal order of the coordinated TPs depends on the context, we can look at few cases in (150), repeated 
from (118). Assuming the two conjuncts to be in an atemporal or simultaneous relation with the context presented in the example, the structure is considered semantically symmetrical because the two TPs can be inverted. Without a salient context, the interpretation of (150) would be of sequential events, therefore of a semantically asymmetric structure. As for a non-ambiguous sequential reading of coordination of TPs, we can see two examples in (151), in which the sequential reading is due to pragmatics.

\footnotetext{
hth $\quad \underline{\text { hth }}$ sp.ips.

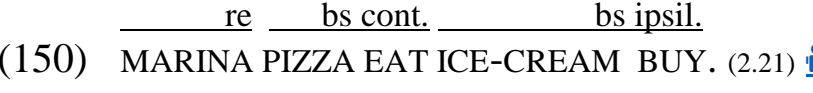

'Marina will eat pizza and will buy an ice-cream.'

Context (atemporal reading): Marina went to a bar, what will she do in there?
}

(151) $\frac{\text { re }}{\text { bs contralat. bs ipsilateral }} \frac{\text { hth }}{\frac{\text { re }}{\text { shn }}} \frac{\text { shn }}{\text { MARINA }}$

(151) a. MARINA CAKE MAKE JORDI SELL. (75.16)

'Marina made a cake and Jordi sold it.' hl contral. hl ipsi. hl contr. re sp. contr. space ipsil.

b. MARINA SANDWICH PREPARE JORDI CL:PILE. (78.10)

'Marina prepared the sandwiches and Jordi piled them.'

Another way to identify the presence of semantic symmetry or asymmetry other than temporal order is to look at contrast. Mauri (2008) identifies opposite, corrective and counterexpectative contrast, leaving aside the contrastive relations between NPs 
described by Umbach (2004) and Hartmann (2015). We can see an example of opposite contrast in (152a), adapted to LSC from Mauri (2008: 86), where the subjects of the conjuncts and the respective VPs are contrasting, also flagged the realization of the NMMs. The example in (152) suggests again the presence of semantic symmetry.
(152) re hl ipsi.
re
JORDI WORK ALREADY MARINA STUDY CONTINUE. (78.03)
'Jordi works already and Marina is still studying.'

space central.

hl ipsi.

The example in (152) show the presence of a symmetry between the conjuncts due to the contrast between them. Repp's (2016) definition is respected also in LSC where "S1 and S2 may be construed as being in a contrastive relation if $\mathrm{S} 1$ contains an element $\alpha$ that can be construed as an alternative to an element $\beta$ in S2, where being construed as an alternative reflects the notions of juxtaposition and comparison". Semantically, then, looking at the temporal relation between the conjuncts and at contrast, both symmetric and asymmetric coordination is found in LSC.

A clear case of asymmetry, though, is found on those borderline cases between coordination and subordination. As we already mentioned in 2.3.3, in SLs it is not straightforward to identify coordination in contrast to subordination due to the scarcity of overt complementizers. In spoken languages, where this distinction can be made easily, authors such as Na \& Huck (1992) and Schmerling (1975), among others, still identify cases of coordination whose meaning and properties are the ones of a subordinate clause. In these 
constructions, the contribution of the two conjuncts is not the same. In $(153 a, b)$ we can see two examples of conditional coordination. The NMMs are similar to the ones used in subordination, where the protasis is expressed with raised eyebrows. No overt marker such as IF is used, though, and the two conjuncts are juxtaposed. In (153c), the sentence expresses a consecutive relation. Weisser (2015), as we saw in 2.1.4, also identifies conative, contiguous, scene-setting and consecutive asymmetric coordination. In LSC, though, these finegrained distinctions would need more study. For the moment we can only see in (153d) that contiguous coordination is available where the verb GO can be bleached, as in spoken languages.

(153) a. GINGER EAT FLU STOP. (78.04)

'You eat ginger and the flu will go away.' hs contr. hl ipsi.

re

b. [RS BEER ONE MORE IX-1 LEAVE]. (78.13) '(You drink) another beer and I'll leave.' eg+bl+hl contr. eg down+hl contr

c. SUN STRONG IX-1 CL:FALL. (78.14)

'The sun was strong and I fainted.'

\author{
hl ipsi. \\ space ipsi. \\ d. MARINA (GO) FRUIT BUY. (79.01) \\ 'Marina went and bought some fruit.'
}

With respect to asymmetric coordination in Weisser's (2015) terms, more material needs to be collected, especially concerning the 
properties in common with subordination, such as the possibilities for extraction. It is clear, though, that this kind of asymmetric coordination as borderline case between coordination and subordination is present also in LSC. A clear case is (153c), where the consecutive relation between the conjuncts is expressed through juxtaposition. In the analysis of coordination in this chapter, the cases of asymmetric coordination presented in (153) will not be included.

\subsubsection{Syntactic asymmetry}

Let us now focus on symmetry and asymmetry from the perspective of syntax. In order to establish an asymmetry between conjuncts that stems from an asymmetric syntactic structure, in spoken languages it is possible to look at the position of the coordinator, at possessee pronominalization, at unbalanced coordination and at binding, even though Progovac (1998) underlines the weakness of this last point as seen in 2.2.1. Due to the lack of case in LSC, unbalanced coordination is not available as a test. Also looking at binding is not straightforward due to the lack of detailed research on quantifiers in LSC. We will use the position of the coordinator and possessee pronominalization to support the need of an asymmetric structure for coordination, even when semantically symmetric.

In section 2.1.3 for spoken languages and in 3.1.1.2 for LSC, we already anticipated that it is possible to establish the position of the coordinator with respect to the conjuncts. LSC shows prepositive coordination, as in (154), in which ALSO and PLUS-Q appear at the beginning of each conjunct. 


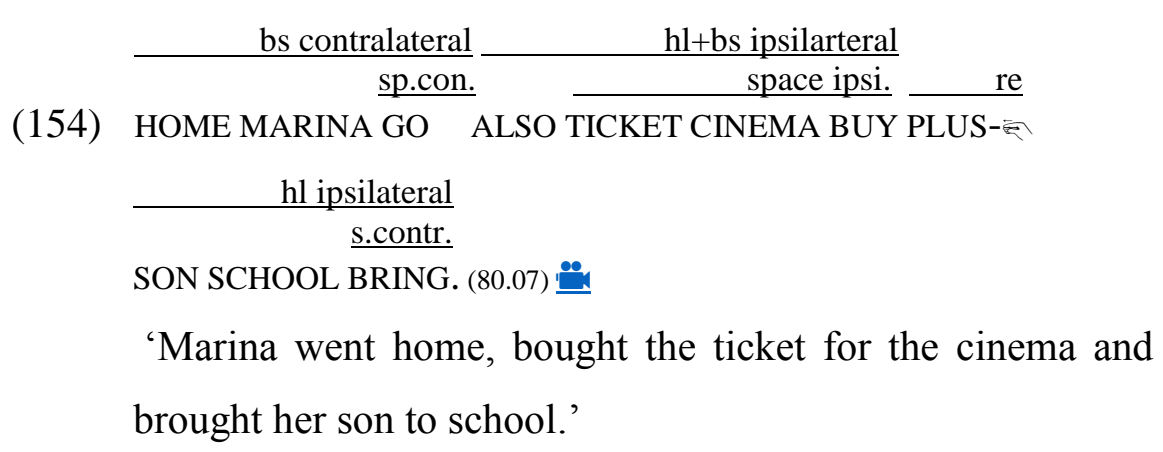

Relying on Zhang's (2010) generalization, which partially states that head final languages showing initial prepositive coordination will have a right-branching coordination, like English, we can conclude that LSC needs an asymmetric right-branching coordination structure, as the one in (155).

(155) Right-branching structure for initial prepositive coordination in head-initial languages.

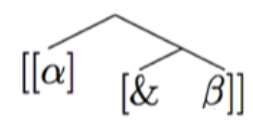

Zhang's generalization needs, then, to be updated assuming that it is the position of the coordinator to determine the directionality of the structure, independently of the word order of the language.

Another argument to support an asymmetric coordination structure in LSC is the need to place the antecedent of a referent in a c-commanding position. For spoken languages, binding with quantifiers has not been considered a reliable tool since the quantifier would c-command the referent, for example a possessive pronoun, only at LF. Examples in (156) in LSC, adapted from Munn (1993) 
and Progovac (1998), are then of no use to look at the binding relation between the conjuncts, as it is also the case with two independent clauses. Moreover, the limited research on quantifiers in LSC could not necessarily confirm the same behavior of quantifiers in spoken languages.

(156) a. GROUP ALL IX-3-PL TEACHER WORK SON IX-3-POSS+++ CHEMISTRY STUDY. (79.14) 는

'Everybody in that group works as teacher and their sons study chemistry.'

b. ALL MAN ALSO DOG IX-3-POSS+++ TOGETHER WALK CL:WALK-LEASH. (80.11)

'Every man and his dog went for a walk together.' As pointed out in 2.2.1, even if these are not cases of actual binding, the possessive pronoun still needs an antecedent and the fact that the two conjuncts cannot be reversed shows that the first conjunct needs to be structurally higher than the second one. It is possible to get to the same conclusion also looking at possesee pronominalization in (157) where it is not possible to invert the two conjuncts.

\section{(157) MARINA IX-3-POSS MUM WORK IX-2-POSS UNEMPLOYED.}

'Marina's mom works and yours is unemployed.' (79.15)

The examples in (156) and (157) confirm again the necessity to have a right-branching coordination structure for LSC, where the first conjuncts is higher than the second one, like in (155). 
The data presented in this section show that in LSC it is possible to find both semantic and syntactic asymmetry and also semantic symmetry in coordinated structures. In semantically symmetric coordination, though, if a syntactic element that needs an antecedent occurs in the second conjunct, like a possessive pronoun, the structure cannot be considered symmetric anymore. We can see an example in (158) where, despite the semantic symmetry, it is not possible to invert the conjuncts because the possessive pronoun needs an antecedent. A sentence with semantic symmetry but asymmetry in the syntax will always need an asymmetric coordination structure.

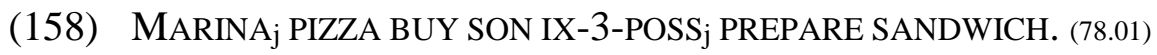
'Marina bought a pizza and her son prepared some sandwiches.'

The properties of coordination in LSC that we examined in this section show that LSC presents semantic symmetry and asymmetry, but I claimed that syntactic asymmetry can always be realized even in semantically parallel structures. Therefore, coordination in LSC will always need a hierarchical structure, more precisely a right-branching one, despite LSC being a head-final language.

Let us look now at more properties of coordination in LSC, through the tests that have been used in the literature to identify this structure in contrast to subordination. The conclusions we reached in this section will be supported also by data presented in the next section, in particular in 3.2.3.1 when looking at ATB wh-extraction. 


\subsubsection{Tests to identify coordination in LSC}

In 2.3.3 we saw the tests used to identify coordination in contrast to subordination in SLs. In LSC, as in other SLs, the lack of declarative complementizers makes subordination and coordination more difficult to tell apart. Tang \& Lau (2012) use extraction, scope of the confirmation tag in yes/no questions and negation to detect the presence of coordination. Van Gijn (2004) and Göksel \& Kelepir (2016), with the purpose of detecting subordination and excluding the presence of coordination, suggest the distributional dependency test and the use of prosody to look at prosodic boundaries between clauses, respectively. Tang and Lau (2012) also consider gapping, being a structure that is used only in coordination in English and in other languages. In LSC, though, as we will see also later in 5.2, gapping can also be realized in clausal adjuncts, therefore this will not be a test used to detect coordination in LSC. In the next subsections we will first look at ATB extraction of wh-elements, then at the scope in yes/no questions and of negation over the conjuncts. We will describe the distributional dependency test in LSC and finally we will present the NMMs that can mark the presence of prosodic boundaries between clauses and therefore coordination.

\subsubsection{ATB extraction: wh-movement?}

In 2.3.3.1 we saw that ASL and HKSL, as also spoken languages like English, are sensitive to the Coordinate Structure Constraint (CSC) by Ross (1967) (see more in 2.2.3), and that the CSC can be violated 
via ATB movement. LSC, as well, is sensitive to CSC, as we can see in (159). In (159a) it is not possible to wh-extract an element belonging to the first conjunct and move it to the right periphery. It is possible, though, to extract out of the second conjunct that is closer to the right periphery where the element is moved, as in (159b). This might be due to the fact that there is $\mathrm{CP}$ coordination and that an element in the second conjunct can move to its right periphery and reach SpecCP. It is not possible to ascribe the grammaticality of (159b) to the absence of coordination, because the typical NMMs of this type of structure are present.

a. *[YESTERDAY MARINA_STUDY] [JORDI COOKIE PREPARE] WHAT $_{i}$ ?

‘*Yesterday, what did Marina study and Jordi prepared cookies?'
$\frac{\text { re }}{\text { hl ipsilateral }}$
b. [YESTERDAY MARINA HISTORY STUDY]

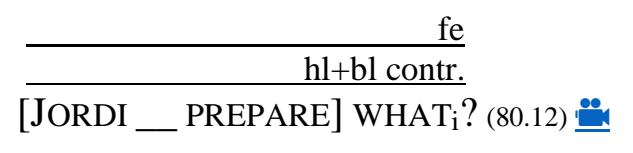

'Yesterday Marina studied history and what did Jordi prepare?'

In (159) we can see, then, that wh-extracting an element from the first conjunct to move it to the right-periphery, as in (159a), it is not possible, respecting the CSC. (159b), though, is grammatical. CSC, then, does not seem to be fully respected in LSC, at least superficially. If we look now into ATB movement, which is supposed 
to save extraction in coordination, we observe that in LSC, as in HSKL, it is not available, as shown in (160a). In (160b), an element is extracted only from the second conjunct, and even though furrowed eyebrows are spreading over the whole sentence, on the first conjunct they mark the presence of a yes/no question and on the second one of a wh- one. In (160c), instead, there is an example of the only strategy available to ask about the same element in conjoined clauses. As in HKSL (Tang \& Lau 2012), as well in LSC it is necessary to have an overt wh-element in the right periphery of each conjunct. The answer to the question can be a single element or two different ones. In the latter case, I refer back to Citko's (2007) analysis presented in 2.2.3 for spoken languages.

(160) a. *MARINA $t_{i}$ BUY JORDI $t_{i}$ READ WHAT ?

'What did Marina buy and Jordi read?

$\mathrm{fe}$

hl+space contr. $\quad \mathrm{hl}$ ipsil.

b. MARINA BUY? JORDI ti READ WHATi ? (46.01)

'Marina bought stuff? and what did Jordi read?'

bl+hl+space contr. bl

c. MARINA $t_{j}$ BUY WHAT JORDI $t_{i}$ READ WHAT ? (45.22)

'What did Marina buy and Jordi read?'

The data in (159) and (160) show that the CSC applies only partially to LSC for wh-extraction. CSC, then, seems not to be a universal constraint and therefore cannot be used as a diagnostic for coordination in LSC. Next, I will focus on the reason why it is possible to have wh-extraction only from the second conjunct to the 
right periphery of the sentence and why ATB wh-extraction is not allowed.

In 3.2.2 data that support a right-branching coordination structure for LSC were presented, and data on wh-extraction constitute a further confirmation. It is possible to justify the data for LSC presented in (160) and repeated in (162) if we assume a rightbranching coordination structure for LSC and the analysis by Wilder (1994) for ATB wh-extraction. For Wilder (1994), in a sentence like (161) from De Vries (2017), wh-movement happens within each conjunct separately and then the wh-element in the second conjunct gets deleted via ellipsis. In (162a) we can see that, in LSC, we need a wh-element overtly produced in each conjunct in order to ask about the same element from both conjuncts, while in English an ATB strategy is used, as attested in the translation. For (162a), we can then assume an internal merge to the right-periphery of each conjunct that corresponds to the direction of SpecCP. Differently than English, ATB movement cannot happen in LSC, as in (162b) and represented in (162b').

(161) [Which book does Peter like _ ] and [which book does Susan hate _ _ ]

$\begin{array}{rr}\text { hl+bl+space contr. } & \text { hl+bl+space ipsi. } \\ \text { fe } & \text { fe }\end{array}$
a. [MARINA BUY WHAT] [JORDI READ WHAT]? (45.22)

'What did Marina buy and Jordi read?'

b. *[MARINA + BUY WHAF 1 ] [ JORDI z READ WHAT $\left._{2}\right]$ ?

'What did Marina buy and Jordi read? 
b'.

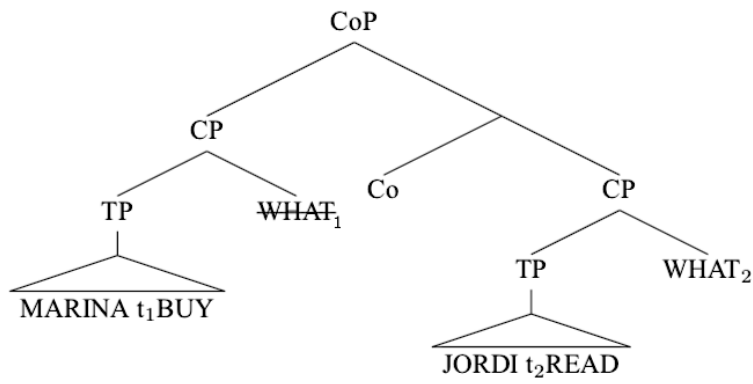

Assuming De Vries's (2017) derivation for ATB, the ungrammaticality of (162b) can be justified by the position of the second conjunct, which contains the overt WHAT. The wh-element WHAT, which has the function of licensing the deletion of the whelement in the first conjunct via identity, moves to the right periphery of the second conjunct and ends up in a lower position than the first conjunct. The overt WHAT is then found lower than the ellipsis site that it should license. Due to the fact that in LSC it is necessary to have a right-branching coordination structure and wh-movement is always towards the right periphery of the clause, it is not possible to have a wh-element scoping over both conjuncts if it is placed in the right periphery of the second conjunct, as in (163). We can see in the tree representation in (163b) of (163a), repeated from (162a), that the second conjunct is lower than the first one, but to license extraction from the first conjunct, it is necessary that a wh-element is present in the SpecCP of the first conjunct, scoping over the position from which the element got moved ${ }^{18}$.

\footnotetext{
${ }^{18}$ I already introduce here the use of coordination phrase (CoP) as category for the coordination structure. We will see the arguments to justify this choice in 3.3.
} 
(163)

a. [MARINA _ BUY WHAT] [JORDI _ READ WHAT]? (45.22)

'What did Marina buy and Jordi read?'

b.

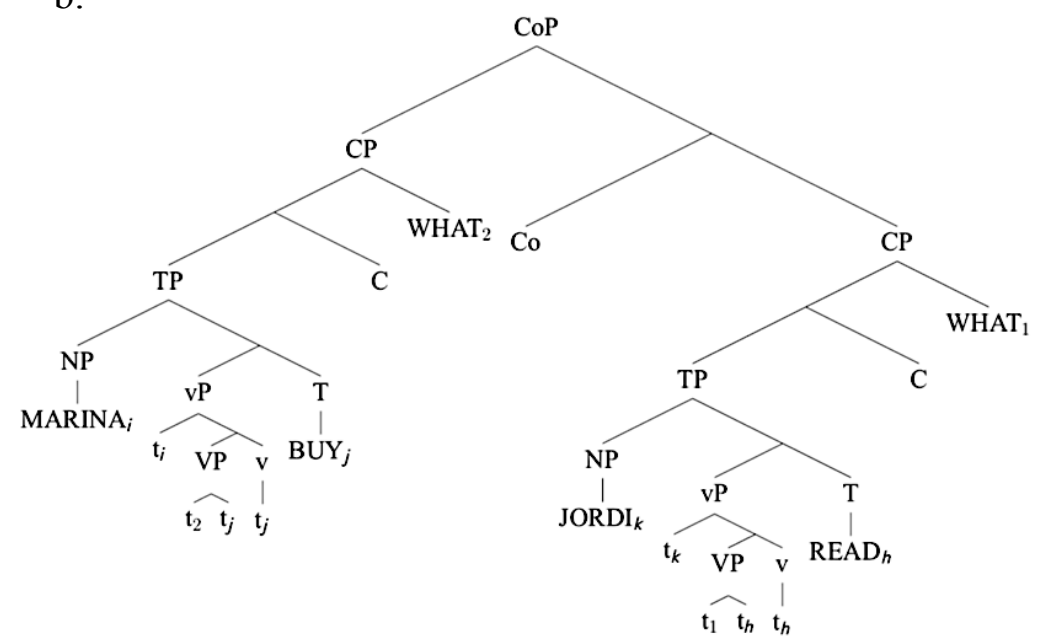

There is more data whose ungrammaticality could depend on the directionality of the coordination structure. Right-Node-Raising (RNR), in which the object of the verb is the same in the two conjuncts but it is produced only in the second one, and backward gapping, another case of RNR, as defined by Johnson (2014), in which the verb is targeted, are not allowed in LSC. We can see an example of each in (164a) and (164b), respectively. The ungrammaticality of both cases can be explained as due to the directionality of coordination structure and to the impossibility of having an element that should scope over both conjuncts in a position where it scopes only over the second one. In 4.2.1 we will present in detail the properties of RNR and we will come back to the issue presented here for LSC in 5.6. 
a. *MARINA LIKE, JORDI LIKE-NOT POPCORN.

'Marina likes and Jordi doesn't like, popcorn.'

b. *MARINA BOOK, JORDI NEWSPAPER LIKE.

‘*Marina book, and Jordi likes newspaper.'

In LSC, the right periphery seems to show peculiar properties that, at least for wh-extraction, can be justified by the directionality of the coordination structure. The need of a right-branching coordination structure for a head-final language like LSC justifies the ungrammaticality of sentences in which a wh-element in sentence final position tries to scope over both conjuncts.

The data in this section show that the application of the CSC and its violation through ATB movement in LSC is not a good way to test the presence of coordination, but it is an important tool to justify once again the presence of a right-branching coordination structure for LSC. Moreover, it is interesting to notice that the CSC cannot be considered a universal constraint.

Let us look now at another test used by Tang \& Lau (2012). We will look at the scope of yes/no questions and negation in conjunction.

\subsubsection{Scope of yes/no questions and negation (in conjunction)}

Tang \& Lau (2012) look at yes/no questions and negation to test if the final confirmation tag in those structures and negation itself can scope over both conjuncts, which would show that they are not 
independent sentences. We will first look at yes/no question and then at negation in LSC.

In LSC, as we have seen also for HKSL in 2.3.3.3, it is possible to use a confirmation tag at the end of the two conjuncts to confirm whether they are both true or false. In (165) we can see that the particle can be RIGHT or a sign that means "yes, no", glossed as $\mathrm{Y} / \mathrm{N}$.

hl+bl contr. hl+bl ipsil.

(165) a. MARINA WIN JORDI LOSE, RIGHT? (75.13)

'Marina won and Jordi lost, right?'

hl+bl contr. hl+bl ipsil. fe $^{19}$

b. MARINA WIN JORDI LOSE, Y/N? (75.14)

'Marina won and Jordi lost, right?'

The fact that the final confirmation tag in a yes/no question scopes over both conjuncts shows that the structures in (165) are cases of conjunction. The typical NMMs used in conjunction spread over the two conjuncts. Interestingly, contrary to the case of wh-extraction where we saw that an element in the specifier of $\mathrm{CP}$ of the second conjunct cannot scope over the two conjuncts, it is then necessary to conclude that RIGHT and Y/N need to be placed in a position higher than the two conjuncts, probably as an adjunct of $\mathrm{CoP}$ or even in a phrase above it.

\footnotetext{
${ }^{19}$ Yes/no questions are characterized by raised eyebrows but in (165b) we can see the use of furrowed eyebrows. For more information about these issues, see Cañas (2018).
} 
As for negation, the same problem of scope seen for whextraction to the right periphery affects also negation. In LSC, negation is placed in the right periphery of the sentence and structurally it is found in a negative phrase (NegP) above TP (Pfau \& Quer 2007), as we can see in (166).

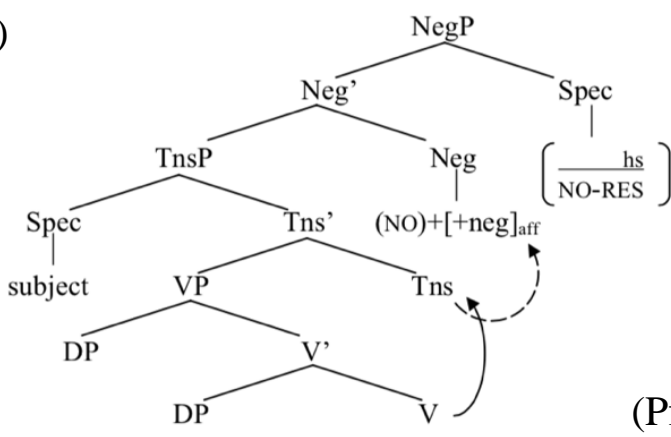

(Pfau \& Quer 2007: 9)

In coordination, though, it is not possible to negate both conjuncts having the negation in the right periphery, as we can see in (167). In (167b) it is clear that the negation in the second conjunct is too low to be able to scope over the first conjunct and negate it. The negation at the end of the second conjunct can only negate the verb in it, in this case BUY in (167a).

a. *TEACHER FOOD COOK SANDWICH BUY NOT ${ }^{20}$.

Intended 'The teacher didn't cook the food and didn't buy a sandwich.'

\footnotetext{
${ }^{20}$ The actual meaning of the sentence is 'The teacher cooked some food and didn't buy a sandwich'.
} 
b.

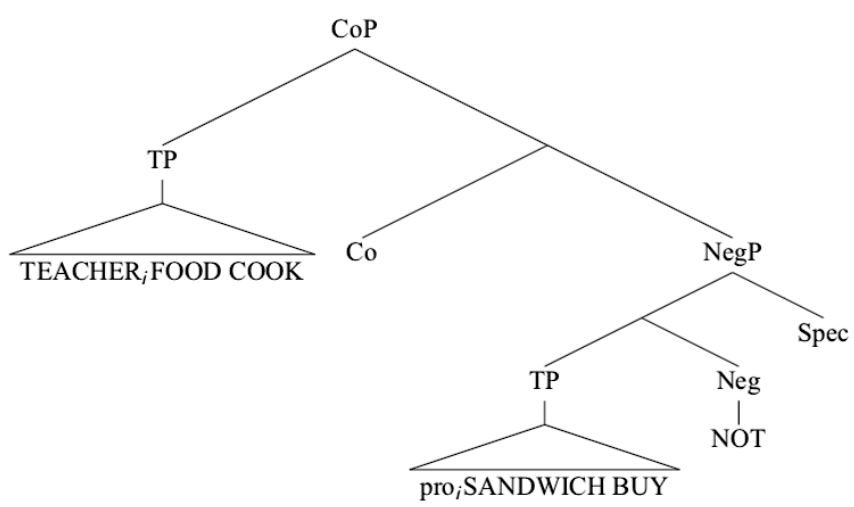

In order to negate both verbs, the negation needs to occur at the end of the first one and the use the sign ALSO-NOT, corresponding to the Spanish tampoco 'either', can extend negation also to the second conjunct, as in (168a). The properties of this sign in interaction with negation will be further explained in 5.2 when talking about the properties of gapping. For now, we will limit the discussion to the fact that this particle can be used to negate the second conjunct when the first one is already negative. Another way consists in using the pronominal element THE-2 we already came across in 3.1.1.2. It can refer back to the subjects of the two conjuncts, as in (168b), or to the two TPs, as in (168c), in order to negate them.

(168) a. TEACHER COOK FOOD NOT SANDWICH BUY ALSO-NOT. (85.02) 'The teacher didn't cook the food and (didn't) buy a sandwich.'

re re

b. MARINA MEAT JORDI FISH THE-2 EAT-NOT. (76.02)

'Marina doesn't eat meat and Jordi fish.' 
re hl+bl+sp. contr. $\quad$ hl+bl+sp. ipsi.

c. TOMORROW PROFESSOR FOOD COOK SANDWICH BUY

re $\underline{\text { hs }}$

THE-2 NO. (76.01)

'Tomorrow the teacher won't cook the food and (won't)

buy a sandwich.'

Out of the two tests proposed by Tang \& Lau (2012) in relation to scope, in LSC is then only possible to apply the one of scope in $\mathrm{y} / \mathrm{n}$ questions.

In the next section we will examine another test, proposed by Van Gijn (2004), which analyzes the possibility of the two conjuncts to stand by themselves in order to distinguish coordination from subordination where one of the two clauses is a dependent one.

\subsubsection{Distributional dependency test}

Van Gijn (2004) uses the distributional dependency test to detect subordination in contrast with coordination. As we saw in 2.3.3.4, it consists in detecting coordination when both conjuncts can be independent clauses. Valin \& LaPolla (1997) point out that cases of cosubordination, or also called semantic subordination or asymmetric coordination, which are sentences that share properties with subordination, also need to be taken into account when looking at dependences between clauses. I will not consider such cases here but for more data about this type of sentences in LSC it is possible to consult section 3.2.2. Looking at a sentence like (169a), repeated from (151), we can see that the two sentences are independent clauses 
and the NMMs clearly indicate that they are coordinated. In (169b), instead, we have a clear case of subordination where the verb SAY is introducing a subordinate clause, from Quer (2016: 207), where the first part of the sentence is not an independent clause.
bs contralat. bs ipsilateral

re hth re shn

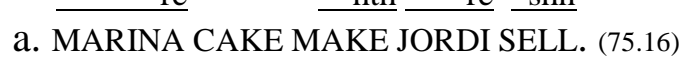

'Marina made a cake and Jordi sold it.'

b. ANNAi 3-SAY-1 $\frac{\text { re }}{\text { IX-3 }}$ i FED-UP LOSE+++.

'Anna told me that she $e_{i}$ was fed up with losing so often.'

The difference between the two sentences in example (169) confirms the possibility to apply the distributional dependency test to detect coordination also in LSC, in contrast to subordination. As pointed out in 2.3.3.4, this test can detect only coordination structures that are semantically symmetric but it has limitations due to the presence also in LSC of asymmetric coordination, as we saw in 3.2.2. Moreover, this test can only be applied with TP coordination.

Another test that can only be used with TP coordination is the one proposed by Göksel \& Kelepir (2016) with the goal of telling apart subordination from coordination.

\subsubsection{Presence of prosodic boundaries}

Göksel \& Kelepir (2016), based on Gökgoz \& Arik’s (2011) work, identify NMMs that mark independent clauses that are therefore also 
used to mark the two conjuncts in coordination in TID. These NMMs are lean backward produced at the end of the final conjunct and head thrust at the end of non-final ones. In LSC it is possible to find similar NMMs to the ones identified for TID. As we can see in (170), repeating (116a) from 3.1.1.1, also in LSC head thrust is used. Moreover, in other examples, it is also possible to have a single head nod, as in HKSL (Tang \& Lau 2012), or a slightly forward movement of the head, very close to a head thrust. As noticed for other SLs (Wilbur 1994, Herrmann 2010 and Pfau \& Quer 2010), also in LSC it is possible to find an eye-blink at the end of the first conjunct as clause boundary marker, as in (170). Comparing eye-blink and headthrust, in LSC, as noticed already by Wilbur (1994) for ASL, head trust is produced on the sign while eye-blinking is found after it.

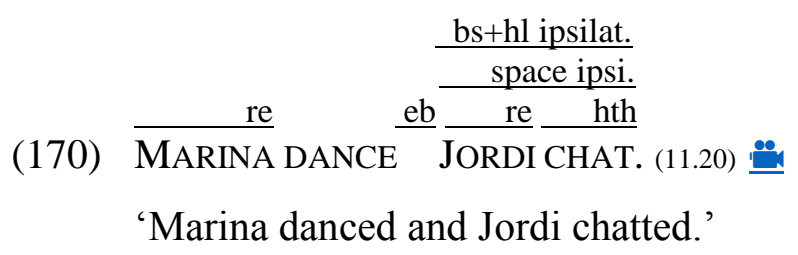

This test proposed by Göksel \& Kelepir (2016) can be used, then, to select coordination of TPs, but it also helps to confirm the presence of other NMMs such as eye-blinking as boundary marker.

Concluding on the tests we presented to identify coordination in LSC, ATB extraction is not an appropriate test due to the different cross-linguistic properties in the application of the CSC. The data discussed, though, give further support for a right-branching coordination structure for LSC. In addition, the impossibility of having negation scoping over both conjuncts is justified by the 
directionality of the coordination structure. Looking at the scope in $\mathrm{y} / \mathrm{n}$ questions is then the only test that can be applied because the scope of the final confirmation tag can be over both conjuncts. We need to assume that the final element is then placed higher than the two conjuncts, probably as an adjunct of coordination phrase (CoP) or in a position even higher. As for the distributional dependency test, it shows some limitations, since it cannot be used for constituents smaller than TPs. Moreover, due to the presence of semantic coordination, it can be considered a test to apply only for semantic symmetric coordination. Finally, determining prosodic boundaries is another test that can be applied with any kind of TPs coordination. This test also shows that the NMMs found in LSC are used in other SLs as well, supporting a cross-linguistic parallel between languages. After establishing the existence of coordination in LSC, we will present next the syntactic analysis of coordination in LSC that will account for the data and properties presented till now.

\subsection{Analysis of coordination in LSC}

In the previous sections we introduced the properties of coordination in LSC. In this one, a syntactic analysis that accounts for the data presented will be provided. I will propose an asymmetric structure whose category will be CoP, standing for "coordination phrase", since it will be used to account not only for conjunction but also for disjunctive and adversative coordination. Moreover, I will follow Munn (1987), whose structure has the conjuncts as specifiers and complements of coordination phrase, but I will adopt Johannessen 
(1998) in the choice of having distinct coordination phrases when there are more than two conjuncts. This will account for the possible use of different coordinators. We will first go through the arguments that justify the asymmetry in the structure summarizing them from the data we saw in the previous sections 3.2.1, 3.2.2 and 3.2.3. Afterwards, we will explain the arguments in favor of having $\mathrm{CoP}$ as category for the phrase that corresponds to a coordinated structure. Finally, we will go through the different manual markers of coordination, focusing first on conjunction, in order to see how they fit in the structure proposed. We will also look at the representation of simultaneous events where both hands are engaged in expressing the conjuncts. To account for this, we will follow Kimmelman (2017), who suggests a multidominance structure. He adopts it also for weak hand holds in list-buoys. Even though list-buoys are not considered coordinators, we will extend Kimmelman's (2017) analysis to LSC with further data on the use of list-buoys in TP coordination that Kimmelman does not discuss. We will first look at data on conjunction and then we will extend the analysis to disjunction and adversative coordination.

\subsubsection{Conjunction}

In 3.2.2 we went through different arguments that support an asymmetric structure for coordination. First, we looked at semantically symmetric and asymmetric structures and then, presenting data on syntactic asymmetry, we concluded that even if semantic symmetry is present in coordination, it is always 
syntactically asymmetric. In examples of semantically symmetric conjunction of simultaneous events where a possessive pronoun is present in the second conjunct, for example, even if there is not necessarily a relation of binding, the two conjuncts cannot be reversed because the pronoun needs an antecedent. This results in the need of always having an asymmetric structure for coordination where syntactic asymmetry rules over semantic symmetry. On the same line we also presented data on possessee pronominalization where, again, the possessive pronoun in the second conjunct needs an antecedent; therefore, the two conjuncts cannot be at the same level. Moreover, the position of the coordinator ALSO or PLUS with respect to the conjuncts supports the need of having an asymmetric coordination structure for LSC too. In fact, as for prosodic grouping, LSC shows initial prepositive coordination $([[\alpha][\& \beta]])$, even being a head-final language. Based on Zhang's (2010) generalization, LSC needs, then, a right-branching coordination structure like English, even if we would expect the head of coordination to be placed on the right of the first conjunct in a left-branching structure like in Japanese. Finally, the directionality of the structure is also supported by the impossibility of having ATB wh-extraction over both conjuncts. Negation cannot scope over the two conjuncts either. This is due to the fact that both elements, the wh-element and the negation, are placed on the right periphery. In a right-branching coordination structure, being the second conjunct lower than the first one, whmovement cannot be licensed to a lower position in the second conjunct and negation, which is placed above TP in the syntactic hierarchical structure of LSC, cannot scope over both conjuncts. The 
use of an asymmetric right-branching coordination structure for LSC is then justified.

Munn (1987), among others, uses an asymmetric and therefore non-flat structure to account for coordination, as we saw in 2.2.4.2.1. The authors supporting a non-flat structure assume that the coordinator belongs to a head whose category, though, depends on the position taken by each author. Depending on the analysis, people proposed BP for Boolean Phrase (Munn 1987, 1993), \&P (Zoerner (1995), CoP (Johannessen 1998) or XP that takes the category of the first conjunct (Camacho 1997; Zhang 2010). For LSC, I propose the need of using a specific category for coordination, CoP, just for the convenience of referring to "coordination phrase", since the structure needs to also account for disjunctive and adversative coordination and not just for conjunction. While authors like Munn (1987, 1993), Zoerner (1995) and Johannessen (1998) support the use of a specific category for coordination, Zhang (2010) defends the need of having the whole coordination structure taking the category of the first conjunct. One of her reasons is related to the possibility in English to coordinate different categories and having $\mathrm{CoP}$ would not put any constraint on the types that can be coordinated. As we saw in 2.2.4.2.1, though, her argument does not hold because it is not only the first conjunct that always has the right category to saturate the verb: the second one might, too. Therefore, if the first conjunct does not represent the category of the two conjuncts, then it is better to have a category that represents their mutual relation instead. In LSC, none of the two conjuncts has a special status in relation to the category the coordination structure needs to assume, since both need 
to be of a category that saturates the verb, as we saw in 3.2.1, differently from English. Moreover, in LSC, NMMs make clear since the beginning of the structure that it is a coordinated one: body or head lean or shift and the use of space mark the presence of coordination from the first conjunct, before the use of a coordinator, differently than spoken languages. This is another reason to posit a specific category for this structure, since it has specific NMMs that identify it.

As for the structure itself, I adopt Munn's (1987) analysis, where the conjuncts are specifiers and complements of a coordination phrase that has a specific category, as we saw in 2.2.4.2.1. In LSC, as in other languages, this seems to be the more appropriate structure due to the fact that a head needs its own phrase. This is in opposition to a structure with the conjuncts being adjuncts of a Boolean Phrase (see 2.2.4.2.3), like the one proposed by Munn (1993), or to the option of having XP as category, like in Zhang (2010). Moreover, a multidominance structure is not sufficient either for spoken languages or for LSC due to its limitations in accounting for asymmetries. Another argument to reject a multidominance approach is related to the fact that in LSC we saw that ATB wh-extraction and scope of the negation on both conjuncts are not allowed, while a multidominance approach could predict this. An asymmetric structure where the conjuncts are specifiers and complements of a coordination phrase can also justify the use of specific NMMs that flag the first conjunct from the beginning of the sentence thanks to the specifier-head relation between the head of CoP and the first conjunct. The NMMs on the second conjunct are then derived by the 
contrast with the ones in the first one. In cases where there are more than two conjuncts, Munn (1987) and Zoerner (1995) do not use distinct coordination phrases, but Johannessen (1998) does. For spoken languages this is more appropriate in order to be able to account for NP sub-grouping and the option of using different coordinators (see 1.2.4.2.1). For LSC, it is important as well to have this distinction, since it is possible to have ALSO and PLUS used in the same sentence as coordinators, as we saw in 3.1.1.2.

The structure proposed by Munn (1987) and followed by Zoerner (1995) and Johannessen (1998), though, has been criticized, as presented in 2.2.4.2. Mainly, Zhang (2010) examines Johannessen's (1998) proposal focusing on unbalanced coordination. According to Johannessen (1998), the specifier-head relation between the head of the coordination phrase and the element in its specifier would license case feature assignment. Zhang (2010) sees this restricted to NP coordination, while in the case of coordinated sentences, the derivation would crash for lack of case assignment. In LSC, due to the absence of morphological case, it is not possible to extend Johannessen's (1998) generalization, or Zhang's (2010) criticism either.

Borsley (2005), as well, finds a structure like the one proposed by Munn (1987) problematic due to the possibility in languages like Italian to have conjunct doubling where the coordinator is produced at the beginning of each conjunct and not just between them (see 2.2.4.2.2). Collins (1988) also uses this argument to propose that each conjunct is the complement of its own \&P, but this structure generates several problems as we saw in 2.2.4.2.2. 
Svenonius (1992), instead, analyzes the coordinator appearing before the first conjunct like an adverb rather than a coordination head (see 2.2.4.2). In LSC, though, this type of structure is not a problem because it is never possible to have ALSO or PLUS appearing before the first conjunct (see 3.1.1.2).

In (171) we can see the structure for coordination in LSC that respects the properties presented till now. We can see that the conjuncts are specifiers and complements of CoP distributed in a right-branching coordination structure, like in (171a). Moreover, when having more than two conjuncts, there are distinct CoP (171b) due to the possibility of having different coordinators in the same sentence. In the head of CoP we have the signs ALSO or PLUS, the two manual coordinators used in LSC. In 3.1, we saw that coordination is mainly encoded via NMMs, thus produced asyndetically, without the use of any manual marker. In this case, we can assume that the head of $\mathrm{CoP}$ is empty. The coordination features are anyway assigned and realized via NMMs, even when the overt coordinator is produced. In the first conjunct, they are expressed thanks to spec-head agreement relation while in the second (and the others) the abstract coordination features have a variable realization, but they always find themselves in prosodic contrast with the ones of the previous conjunct. As we saw in the previous sections, the NMMs that spread on the conjuncts are not systematically produced with body, head and space all aligned toward the opposite direction of the ones in the previous conjunct. There is always contrast, though, at least between the orientations of the body or of the space. It is important, then, to tell apart the way of 
accounting for abstract coordination features in the syntactic tree and their actual realization in signing.

a.

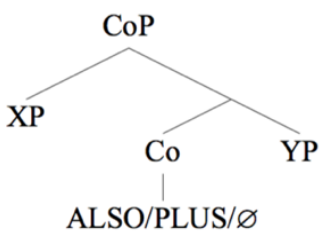

b.

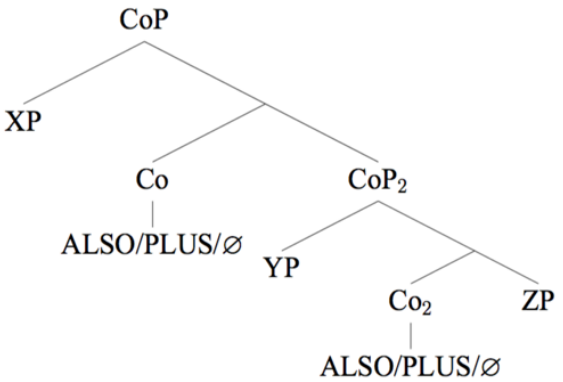

As for ALSO or PLUS, we can then confirm that they are placed in the head of CoP, which can be left empty if no manual marker is used. In example (172a) and its representation in (172b), we can see that the coordination is a right-branching structure but the material in the conjuncts remains head-final. As for NMMs spreading over the conjuncts, whether there is an overt sign or not used, the ones in the first conjunct are licensed by the spec-head relation. As for the ones spreading on the other conjuncts, they distribute in the structure in contrast to the ones on the previous one. In the actual realization, we can see that the contrast is not systematic towards opposite directions for each conjunct but even if in the first two conjuncts the head leans towards the same side, there is contrast in space. Between the second and the third, instead, it is the orientation of the head that is in opposition. 
hl ipisl. sp.cont.

$\underline{\operatorname{shn}}$

hl ips.

$\underline{\operatorname{shn}}$

(172) MARC CAKE BAKE // ALSO MARINA PIZZA EAT // $\overline{\text { ALSO IX-2 }}$

hl ipsi. $\quad \underline{\text { shn }}$

SANDWICH PREPARE. (1.25)

'Marc baked a cake and Marina ate a pizza and you prepared a sandwich.'

b.

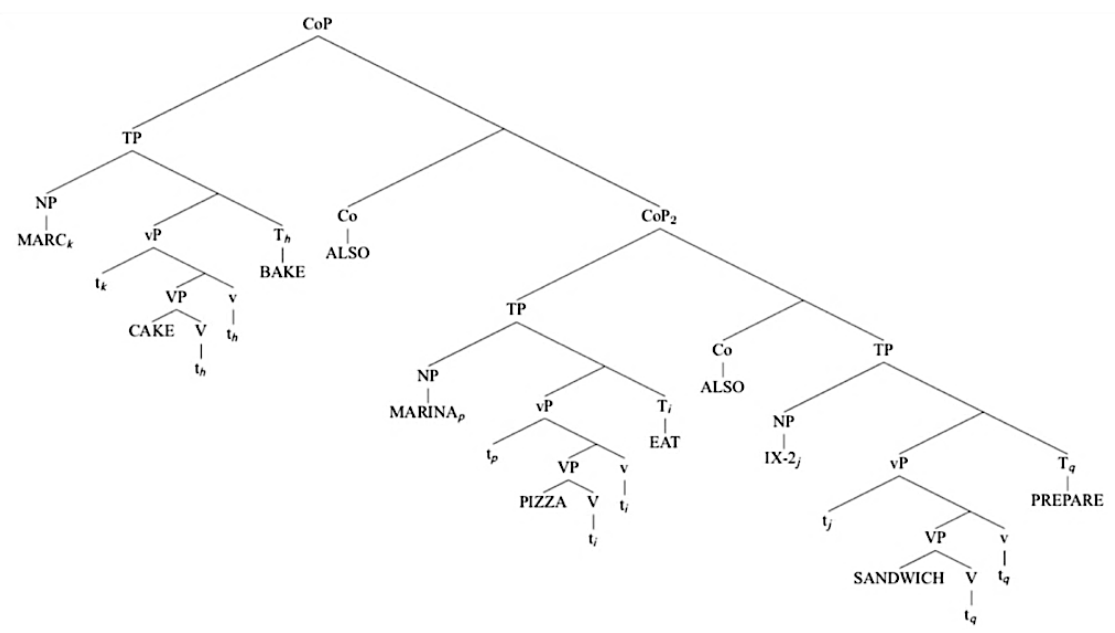

The structure in (172b) can be used to represent all the cases of conjunction, whether they show semantic symmetry or asymmetry, since anyway syntactic asymmetry is always present, as we specified in 3.2.2. While ALSO or PLUS can be placed in the head of CoP, this is not the case for list-buoys and for THE- 2 and BOTH either, though. List-buoys, following Kimmelman (2017), will be considered instances of frame-setting topics. THE- 2 and BOTH, instead, as we saw in 3.1.1.2, are considered pronominal elements. These last two, then, will not be included in the analysis due to their anaphoric nature and their lack of contribution as coordinators. I will leave their analysis for future research. As for list-buoys, instead, since they are very 
common in introducing conjuncts and show interesting properties in simultaneity, we will incorporate them into the analysis. Based on Kimmelman (2017), it will also be possible to account for other cases of simultaneity in coordination in LSC where two events are expressed at the same time either with both hands moving at the same time or with the non-dominant one holding while the dominant one produces another sign. For structures in which simultaneous events are expressed using both hands at the same time, having each one realizing a conjunct, the representation in (172b) is not enough. In (173) we can see two examples of this type of strategy to express simultaneous events. In (173a) both hands keep the movement of the sign while in (173b) the non-dominant hand is held still while the dominant one keeps the full movement of the sign for CL:STIR.

$$
\text { bl+space ipsil. } \quad \begin{aligned}
& \text { alternation head shift+eye gaze } \\
& \text { space ipsi }
\end{aligned}
$$

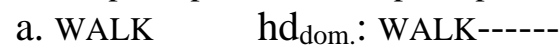

$$
\text { hd }_{\text {non-dom. }} \frac{\text { hs contr. }}{\text { CL:EAT---- }}
$$$$
\text { (78.11) }
$$

'I was walking and eating (looking around)'.

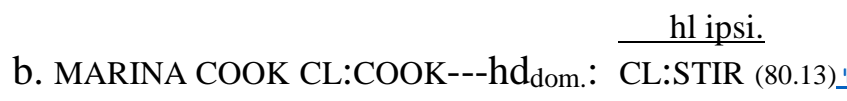

$$
\mathrm{hd}_{\text {non-dom. }}: \frac{\text { space+eg contralateral }}{\text { WATCH--------------- }}
$$

'Marina was cooking and watching (something).'

In (172b) the linearization of the signs does not presuppose that the two hands are producing each one a different conjunct at the same time. For this type of structure it is necessary to add a further step in the derivation, that is external remerge (Citko 2005; van Riemsdijk 
2006; de Vries 2009). I will follow Kimmelman's (2017) analysis, where he uses this strategy to also account for simultaneous structures in RSL and I will adapt it to LSC taking into consideration also some data on list-buoys referring to whole TPs that he does not mention.

\subsubsection{Coordination of simultaneous events}

As we presented in the previous section and in 3.1.1.2 for LSC, as in other SLs such as RSL, simultaneous events can be expressed using both hands simultaneously. There are two types of such phenomenon, depending on the behavior of the non-dominant hand that can keep the movement of the sign or be held, maintaining handshape and location without performing the typical movement of the sign. In the latter case, such phenomenon is referred to as "weak hand hold". Kimmelman (2017) proposes an analysis for this in RSL, using de Vries' (2009) analysis for multidominance structures that involves external remerge. In his analysis, Kimmelman (2017) refers to examples like the one presented below in (174). From the glosses we can see that at the beginning of the sentence both hands are expressing the holding of a suitcase each. After that, the nondominant hand keeps holding the sign corresponding to carrying a suitcase while the dominant hand expresses that holding the other suitcase it is not necessary and it is thrown away. The important aspect is that the non-dominant hand holds the sign from the beginning through the whole sentence but the event takes place only once. 
(174) H1: CL:CARRY1. SUITCASE NEED.NOT. THROW.

(RSL)

H2: CL:CARRY

'He carries [the suitcase and the cage]. He does not need the suitcase. He throws it away.'

(Kimmelman 2017: 52)

In order to account for the simultaneous production of two TPs, Kimmelman (2017) adds to the derivation the presence of External Remerge that results in a multidominant structure where one node is dominated by at least two other nodes and merged to form a singlerooted tree in a coordination structure.

External Remerge is also called parallel merge (Citko 2005) and used to account in spoken languages for phenomena such as ATB extraction or Right Node Raising (RNR), among others. Differently than simple Merge, in which two roots are joined, and Internal Merge, which takes place when movement is realized with a root joining a constituent it is part of, External Remerge joins two roots that belong to different constituents. We can see an example of representation of external remerge in (175), where the root $\mathrm{B}$ is externally remerged to the constituent E. Considering concrete examples, in (176a) we have a case of RNR and in (176b) one of ATB phenomena. The element shared by the two conjuncts in the two examples is argued by de Vries (2017) to be externally remerged with the first conjunct creating a multidominant structure. 
(175) External Remerge

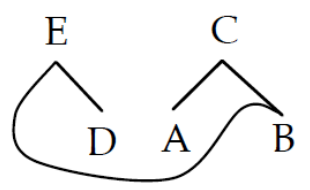

(176) a. Peter likes, and Susan hates, this book.

b. Which book does Peter like and Susan hate?

(de Vries 2017: 1, 2)

This type of structure is relevant to account also for simultaneity in SLs. In the example we saw in (174) for RSL, repeated below in (177), Kimmelman (2017) underlines that the TP produced by the non-dominant hand is held over the whole sentence, but it does not belong to the three sentences because it does not happen three times, it only happens once at the beginning. What is shared between the sentences is, then, a zero scene-setting topic (sst) that specifies the temporal and spatial framework of the situation in the sentence, corresponding to "at this time and place". Looking at the structure in (178), proposed by Kimmelman (2017), the scene-setting topic is placed in the left periphery of the TP that is held over the sentence and also in the left periphery of the series of conjuncts produced by the dominant hand. This is, then, the node that gets externally remerged, realizing a multi-rooted coordination structure, giving the interpretation that the first two CPs in the structure happen simultaneously, sharing the same time and place. 
(177) H1: CL:CARRY1. SUITCASE NEED.NOT. THROW.

H2: CL:CARRY

'He carries [the suitcase and the cage]. He does not need the suitcase. He throws it away.'

(178)

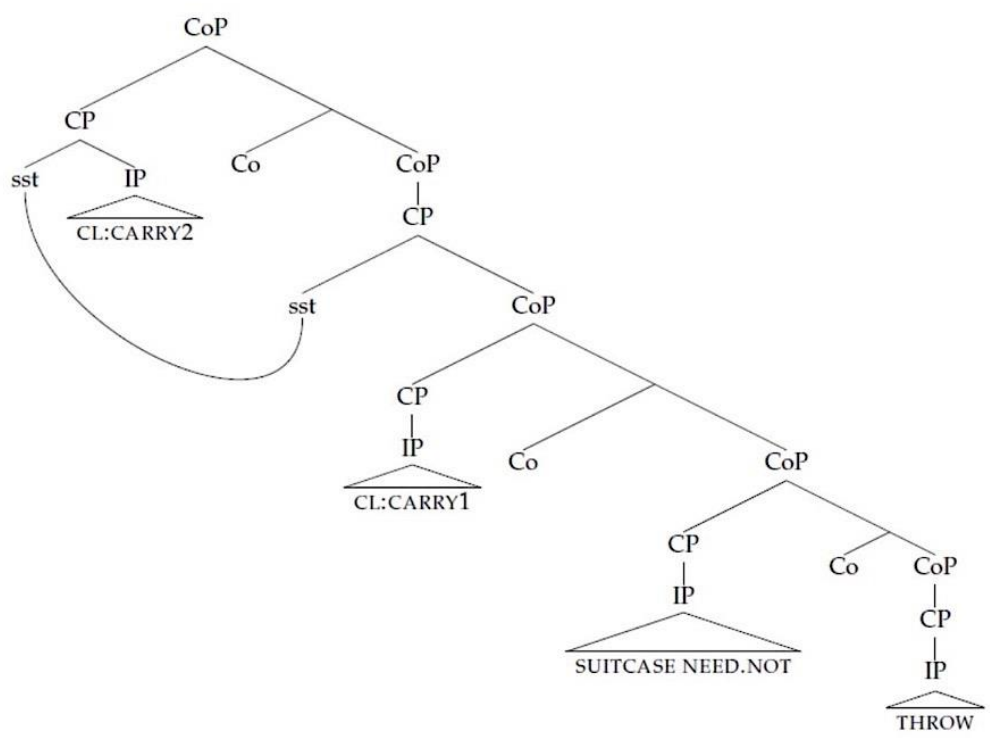

Kimmelman (2017) also focuses on the linearization rules that come with a multidominant structure, following de Vries (2009) and proposing a further rule that accounts for the SL modality-specific realization of a zero element, the scene-setting topic, as shared element.

The relevant part of the linearization rule proposed by de Vries (2009) and selected by Kimmelman (2017) for his discussion is the one in (179). 
(179) Spell-Out of Remerged Nodes (de Vries 2009: 381)

A node with more than one parent is linearized if and only if (i) the current parent is not dominated by any other parent, and

(ii) - every parent has been traversed, or

- the current parent dominates every other parent that has not been traversed.

In (180) we can see a representation of it, where $\alpha$ is spelled-out in the right-most position after $\gamma 1, \gamma 2$ and $\gamma 3$, which do not dominate each other, as specified in (179ii), have been traversed. Also (179i) is satisfied since $\gamma 1$ is not dominated by any other parent.

(180) Linearization of External Remerge (de Vries 2009: 379)

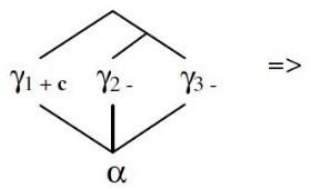

omit $\alpha$

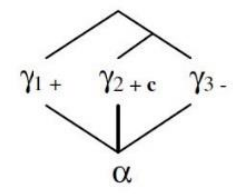

omit $\alpha$

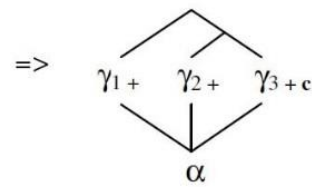

spell out $\alpha$

Based on this proposal, Kimmelman (2017) gives the linearization rules that account for the data in RSL that show simultaneity, and for cases where the shared element, the zero scene-setting topic in the example we saw in (174), is not overtly realized. In (181) we can see his proposal for RSL. 
(181) Spell-Out of Externally Remerged Nodes in RSL modified (Kimmelman 2017: 53)

(i) A node with more than one parent (and the parents of which do not dominate each other) is linearized on the second tier (= the weak hand) when the first parent has been reached.

(ii) If the node is phonologically empty, the next node in the line gets linearized on the second tier.

Considering again the example (174) for RSL repeated here in (182), we can see that the shared node is the scene-setting topic that, being phonologically empty, gets linearized on the second tier, the weak hand. CL:CARRY2, the node that follows the scene-setting topic is linearized on the second tier as well, following (181ii).

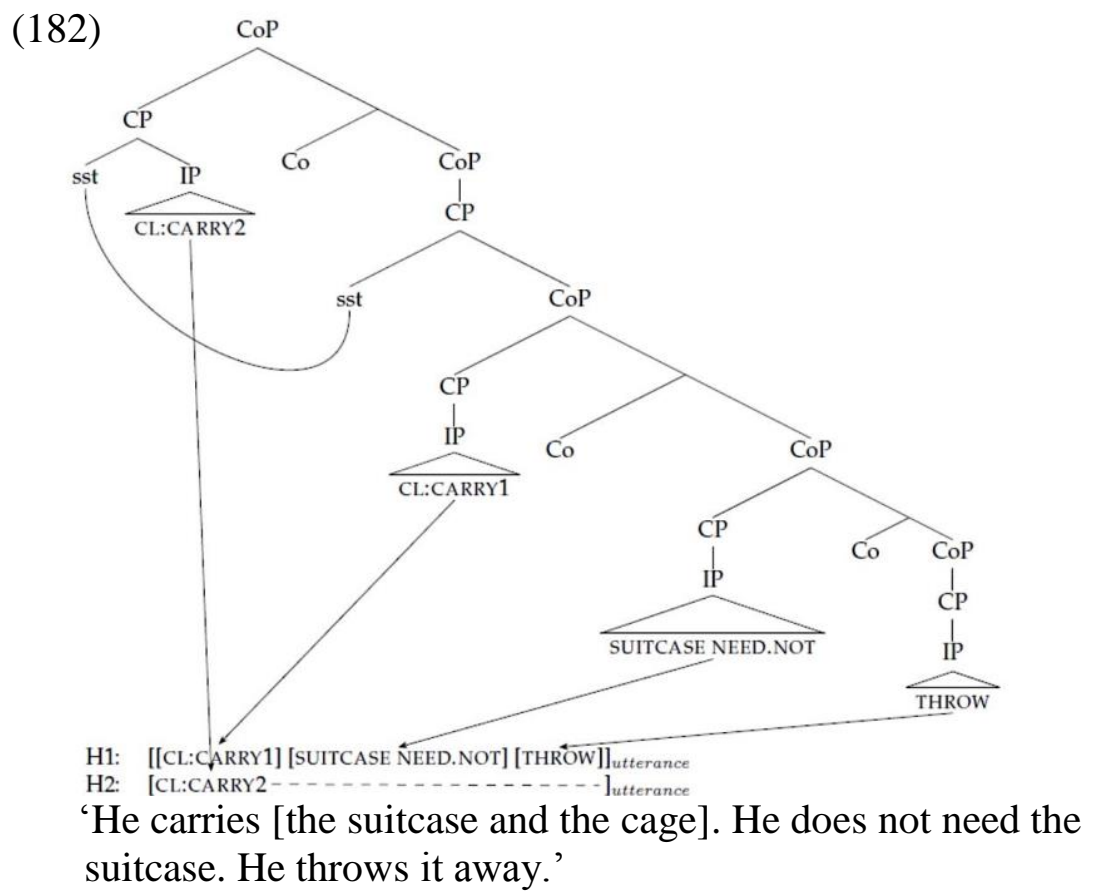


Kimmelman (2017) focuses on the issue of synchronization of the signs as well, defining it independent of linearization rules. In (183) we can see his "prosodic alignment of tiers for RSL", where the second point (183ii) relates to (183i) underling the fact that the holding of the sign on the non-dominant hand is kept till the end of the last sign of the utterance signed by the dominant one. Point (183iii) is specifically related to cases where the non-dominant hand is held, but it does not apply to cases that we will discuss for LSC where the non-dominant hand keeps the movement of the sign, therefore it is not held.

(183) Prosodic alignment of tiers in RSL (Kimmelman 2017: 45)

(i) If two tiers are active, each one is mapped onto a single utterance.

(ii) If two utterances appear on two tiers, their right edges should be aligned.

(iii) If one sign has to constitute an utterance, it is realized as a hold, not as repetition of the full sign. If more than one sign is linearized on the second tier, the last one is realized as a hold.

With respect to signs synchronization, Kimmelman (2017) considers it a prosodic matter, describing prosody as "a component of grammar containing rules of synchronization of the two hands".

Let us look now at LSC data where there is holding of the non-dominant hand to express the simultaneity of events. In example (184), the verb WATCH is produced while the verbal classifier for 
CL:COOK is signed and then held. In LSC the two simultaneous TPs share time and space, just as in the example from RSL (174), but it is also important to notice that the simultaneity of the two events always shows the presence of role shift (RS). Therefore, it seems that the presence of a zero scene-setting topic (sst) can be supported by the presence of RS. In this way it also helps to account for the alignment in the production of the two signs. In the structure in (185), used to represent example (184), it is possible to see that, marking the presence of RS, it is also possible to better mark that the verbal classifier CL:STIR and WATCH are physically produced simultaneously, even though the exact beginning of STIR is difficult to mark. As for RS, we can assume Quer's (2005) approach, based on Lillo-Martin's (1995), where she proposes a covert Point of View Predicate (POV) to introduce RS. This POV has the function of selecting an embedded $\mathrm{CP}$ that binds its operator in SpecCP and whose complement is the sentence produced under RS. Quer (2005), instead, in order to account for both quotational and non-quotational instances of RS, suggests that RS instantiates a Point of View Operator (PVOp), rather than a Point of View Predicate (POV). PVOp is materialized through the production of the NMMs realized with RS and spread over the structure. PVOp is argued to be a covert operator over contexts and does not select a CP but it is found on top of the whole structure, in the head of a Speech Act Phrase (SAP). In the LSC example (184), where simultaneity is involved, what is introduced by SAP is a coordination phrase where the two conjuncts, two TPs, share the same time and space realized through a scenesetting topic. The use of External Remerge marks the part of the 
structure that is physically produced simultaneously. If there was no holding of the verb on the non-dominant hand, no external remerge would be needed. The structure that represents (184) is the one in (185).

hl ipsi.

(184) MARINA COOK [RS CL:STIR---hd dom.: CL:STIR (80.13)

$$
\text { hd }_{\text {non-dom: }}: \frac{\text { space+eg contralateral }}{\text { WATCH--------------- }}
$$

'Marina was cooking and watching (something).'

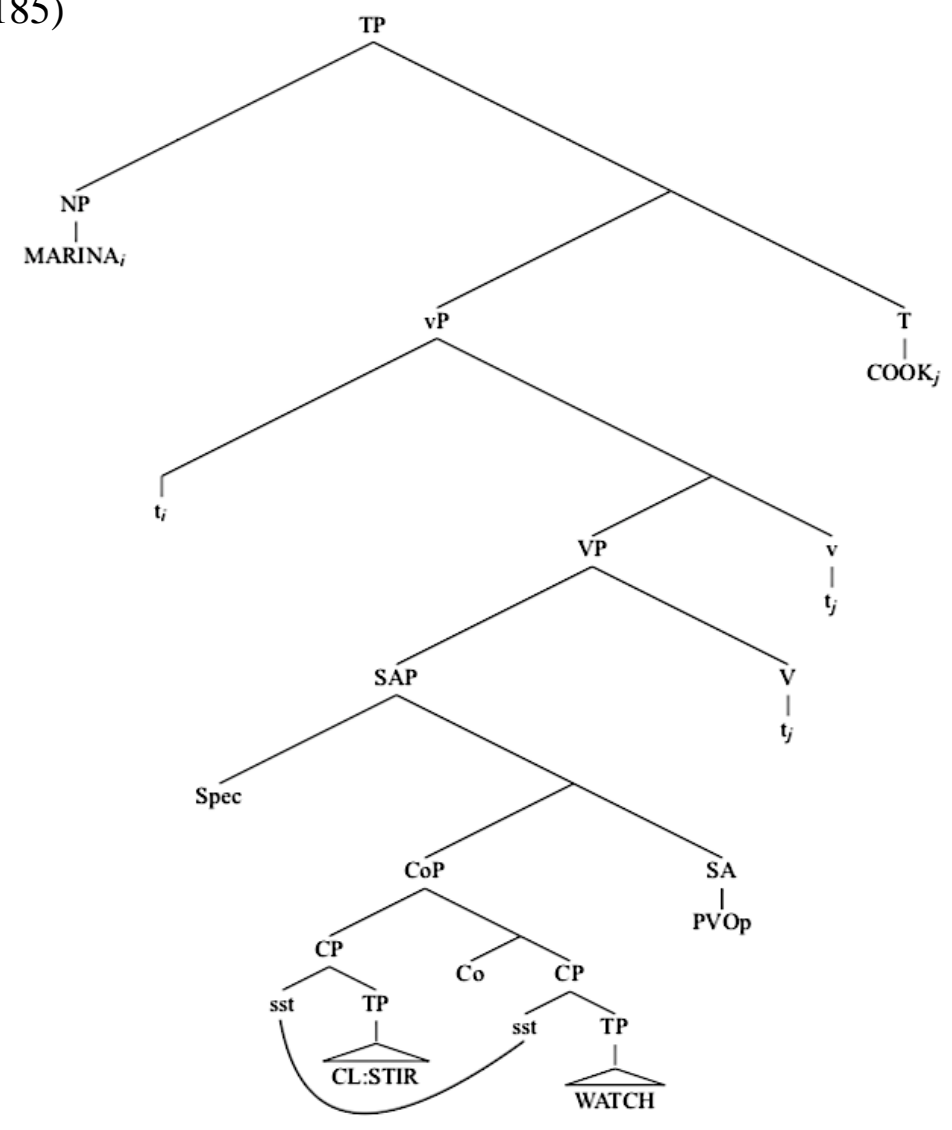


Kimmelman (2017) does not consider cases of simultaneity in which the non-dominant hand keeps the movement of the sign. In LSC, though, as we saw in 3.1.1.1, it is a structure that can be produced. When two TPs are produced simultaneously what is shared is the zero scene-setting topic, so it does not affect the structure whether the nondominant hand is held or keeps the movement of the sign. As pointed out by Kimmelman (personal communication), it is possible to adapt the prosodic alignment of tiers rules that we saw in (183). Point (183iii) only mentions the presence of holding of the verb on the nondominant hand. In LSC, it would then be necessary to adapt it like in (186iii).

(186) Prosodic alignment of tiers adapted to LSC (adapted from Kimmelman 2017: 45)

(i) If two tiers are active, each one is mapped onto a single utterance.

(ii) If two utterances appear on two tiers, their right edges should be aligned.

(iii) If one sign has to constitute an utterance, it is not necessarily realized as a hold, the non-dominant hand can also keep the lexical movement of the sign. If more than one sign is linearized on the second tier, the last one can be realized as a hold or keeping the movement of the sign.

Considering now example (187), the verbal classifiers WALK and EAT are produced under RS simultaneously. It is possible to assume, as in the previous example, that they are put in a $\mathrm{CP}$ coordination structure 
where the scene-setting topic is placed in the specifier of $\mathrm{CP}$ and externally remerged, realizing a multidominant structure that represents the simultaneity in the production of the two TPs. Moreover, the presence of RS is again introduced by the the PVOp in the head of SAP. We can see the representation of (187) in (188). The absence of an overt verb above SAP is due to the fact that RS does not necessarily need to be introduced by a verb.

bl+space ipsil.
alternation head shift+eye gaze
$\frac{\text { space ipsil. }}{\text { WALK------ }}$

hs contr.

$$
\text { hd }_{\text {non-dom. }}: \overline{\text { CL:EAT---- }}
$$

'I was walking and eating (looking around)'.

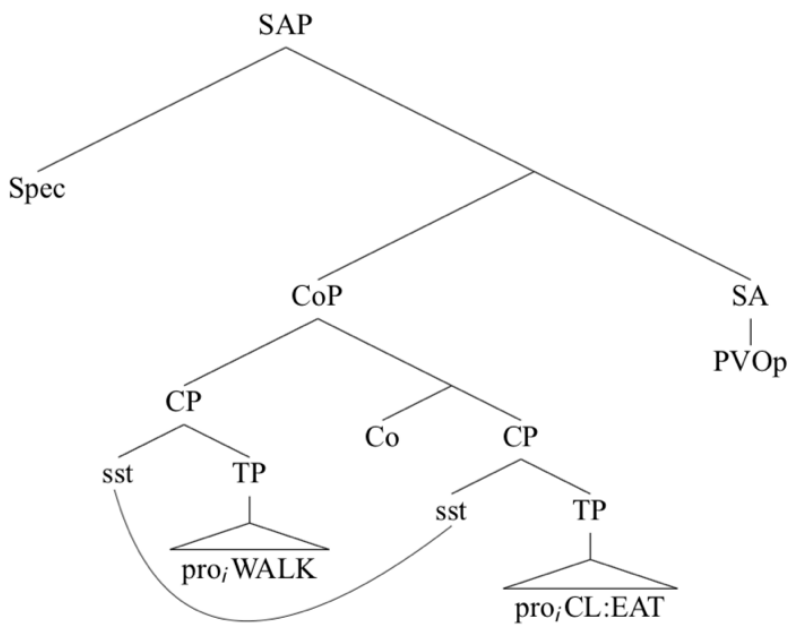

In order to account for simultaneous events in LSC it is possible, then, to adopt Kimmelman (2017) with some adaptations. The first needs to be done to the prosodic alignment of tiers rules that need to 
include also the possibility of having the non-dominant hand keeping the movement of the sign. The second one is to the structure itself: in LSC, simultaneity of events expressed through simultaneous use of both hands always features role shift (RS). Thus, RS needs to be represented in the structure and the sharing of time and space with scene-setting topic (sst) can be realized in the scope of SAP through external remerge. The lack of simultaneous production of the events on both hands would end in the absence of external remerge of the sst. As for the linearization rules, due to the similar properties that we saw between RSL and LSC, it is possible to use Kimmelman's (2017).

A similar analysis that uses External Remerge to account for simultaneous TPs can also be adopted to account for list-buoys, as proposed by Kimmelman (2017). In the next subsection we will go through the analysis for list-buoys in LSC and discuss cases where the non-dominant hand is held in a simultaneous fashion and where not.

\subsubsection{List-buoys}

The analysis proposed by Kimmelman (2017) for cases of simultaneity was first proposed to account for cases of weak hand holds in the use of list-buoys. As specified in 3.3.1, list-buoys in LSC are not used as a coordinator but rather as pronominal pointers to the referents in the conjuncts, either NPs or TPs. As for their production, the non-dominant hand can hold the total number of referents through the whole sentence while the dominant one realizes the pronoun 
pointing at the referent in the conjunct on the non-dominant hand instead of in space. Another strategy consists in having the total number of referents on the non-dominant hand, but not holding them through the whole sentence; in that case, the list buoy is only used in combination with the dominant hand at the beginning or at the end of each conjunct. In both cases, the total number of referents that will be produced is already established since the beginning of the sentence. We can see an example of each strategy in (189a, b), respectively. There is also a third way to refer to the referents in the conjuncts, but it is just a listing strategy and does not involve listbuoys per se.

hl contralateral

a. hd $d_{\text {dom: }}$ (LIST2)-IX-a MARINA PIZZA CL:BAKE

hl ipsilateral

re sp. ipsi.

(LIST2)-IX-b JORDI CAKE CL:BAKE

hd $d_{\text {non-dom: LIST2 }}$ (80.08)

'On the one hand, Marina baked a pizza and on the other, Jordi baked a cake.'

re bl+sp.contr. re

b. LIST2-IX-a MARC CAKE CL:BAKE LIST2-IX-b MARINA PIZZA bl+sp. ips.

CL:BAKE. (1.17)

'On the one hand, Marc baked a cake and on the other, Marina baked a pizza.' hl+bl+space ipsil. re

c. LIST1-IX-a MARINA CAKE CL:BAKE LIST2-IX-b JORDI PIZZA sp.ipsi.

CL:BAKE. (81.08) 는

'On the one hand, Marina baked a bake and, on the other, Jordi baked a pizza.' 
In the three strategies, only the first one, (189a), shows simultaneity and therefore the need of having a multidominant structure where the shared node is LIST2. In (190), we can see one of Kimmelman's (2017) examples where list-buoys is used to refer to NPs.

(190) H1: IX-a DAVIDENKO D-A-V-I-D-E-N-K-O. IX-b N-A-D-I-A IX-b. IX-c R-I-T-A

H2: THREE.LIST-

'Of the three of them, the first one was Davidenko, the second one Nadia, and third the third one was Rita.'

(Kimmelman 2017: 30)

The node shared in (190) is THREE.LIST, which is produced at the beginning and held through the whole sentence. Kimmelman (2017) argues that list-buoys are functionally similar to scene-setting topic and they can be defined as frame settings: following Krifka (2008), they specify what the signer is talking about. A frame setting introduces a frame in which a set of propositions holds (Krifka 2008). The representation used by Kimmelman (2017) for (190) is, then, the one in (191), where the frame setting represented by THREE.LIST is placed in the specifier of $\mathrm{CP}$ of each conjunct and external remerge takes place. 


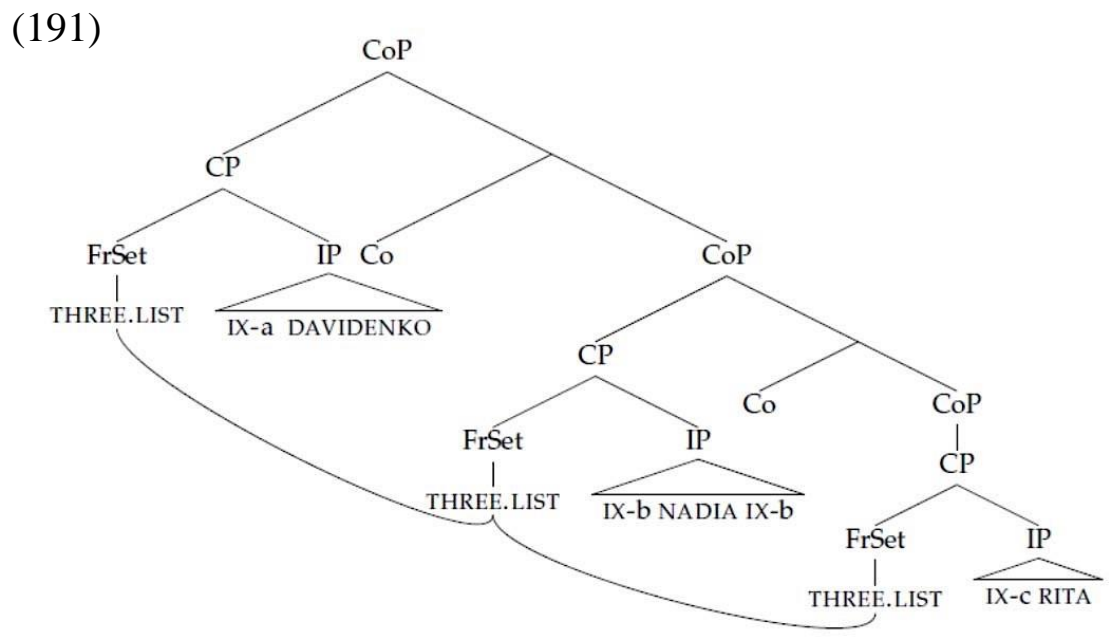

For LSC we can use a similar structure such as (191), where LIST2 is held over the two conjuncts. I agree with Kimmelman (2017) that the nature of the index that is used in combination with the LISTX produced by the non-dominant hand is a pronoun, carrying reference to the event or entity in the conjunct. List-buoys, in fact, can only appear in conjunctive and disjunctive coordination, they are not available in adversative coordination, as we can see in the example below in (192), where the relation between the conjuncts is counterexpectational.

*LIST2-IX-a JORDI VERY-TALL BUT LIST2-IX-b BASKETBALL PLAY VERY-BAD

\#'On the one hand, Jordi is very tall but, on the other, he's very bad at playing basketball.'

As we will see in 3.3.3, adversative coordination with counterexpectational BUT does not involve the presence of a limited set of referents or propositions to which to refer. Therefore, the data 
in (192) confirm that the pronoun in list-buoys actually refers to the subject or the content of the whole proposition in the conjunct and not to the syntactic conjunct itself, meant as mere syntactic unit. The need of having a set of referents to which the pronoun in list-buoys is referring to is confirmed.

In LSC, though, it is also possible to encode temporal order through list-buoys, but only when the the - -handshape is accompanied by puffed cheeks. I argue that, in this case, we are not dealing with a pronoun but rather with a temporal adverb. In the syntactic representation, the adverb will appear in SpecCP modifying the whole TP. Differently than the list-buoys used as pronoun, the adverb will be only found at the beginning of the conjunct.

When list-buoys are used, the signer in principle already knows how many referents s/he will produce. Therefore, the total number of referents is encoded in the sentence and for this reason it can be assumed to scope over the whole coordination phrase. This choice can also be justified by the number of referents that can be introduced before the conjuncts are produced. When the total number is not inferred, it is anyway realized scoping over the whole sentence but as a covert element. Whether it is overt or not, I argue that LISTX always needs to scope over the whole structure. For RSL, Kimmelman (2017) suggests this option in the case where no simultaneity is performed. In the case of the presence of simultaneity in LSC, the total number of referents is, then, externally remerged in the specifier of $\mathrm{CP}$ of each conjunct, since it is actively engaged at the beginning of each one. Differently from Kimmelman's (2017) 
approach, though, I argue that the pronoun is not part of the TP, but it is considered a topic element that introduces it. The example in (193), repeated from (189a), can then be represented as in (194).

hl contralateral

re sp. contr.

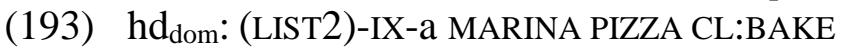

hl ipsilateral

re sp. ipsi.

(LIST2)-IX-b JORDI CAKE CL:BAKE

hd $d_{\text {non-dom: LIST2 }}$

(80.08)

'On the one hand, Marina baked a pizza and on the other, Jordi baked a cake.’

(194)

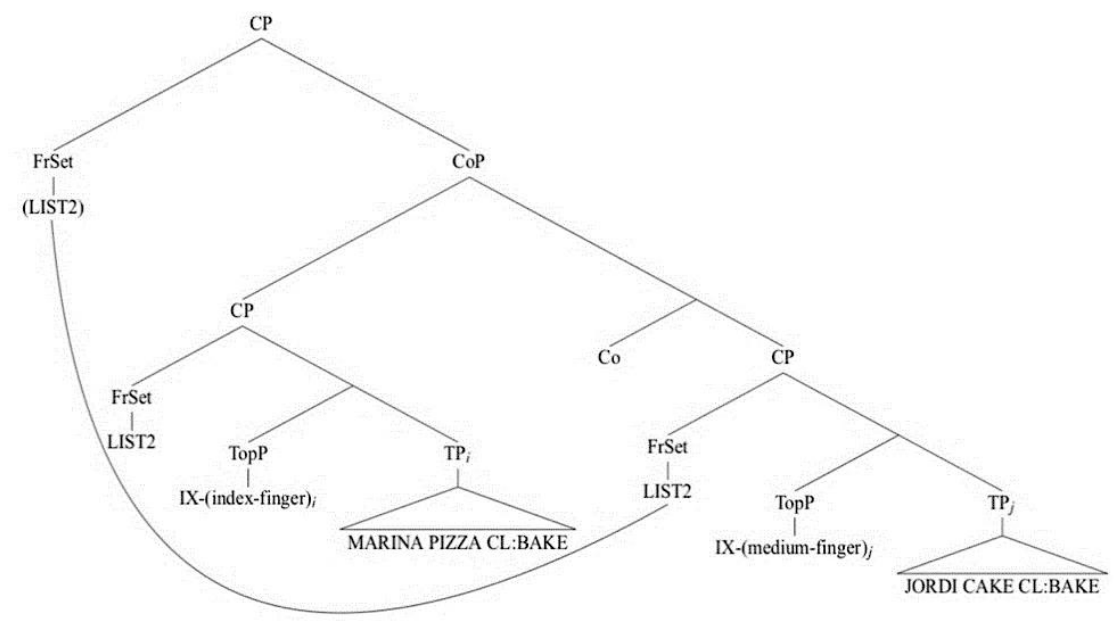

In cases where list-buoys are produced without holding the nondominant hand, LISTX is placed above CoP scoping over the whole sentence, overtly or covertly, and then it is repeated in each conjunct, without the need of a multidominant structure. Its nature, though, will 
still be of frame setting. We can see the representation of example (195), repeated from (189b), in (196).

$\frac{\text { re }}{\text { LIST2-IX-a MARC CAKE }} \frac{\text { l+sp.contr. }}{\text { CL:BAKE }} \frac{\text { re }}{\text { LIST2-IX-b MARINA PIZZA }}$ bl+sp. ips.

CL:BAKE. (1.17)

'On the one hand, Marc baked a cake and on the other, Marina baked a pizza.'

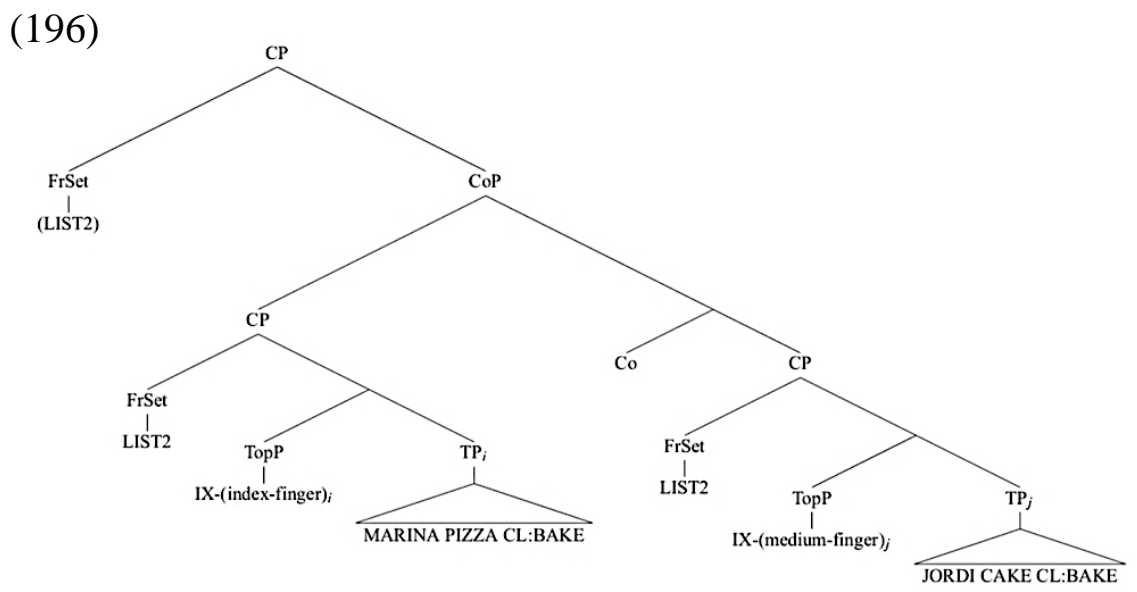

The only case in which LISTX does not scope over the whole structure is in cases where the total number of referents is not performed on the weak hand. This happens in cases where this amount is not encoded in the meaning of the sentence, like in example (197). This cannot be considered a case of list-buoy, but a simple enumeration where the pronoun is produced on the weak hand that corresponds to the number of the listed referents. In the tree in (198) we can see that LISTX does not appear as a frame setting element, since the signer is 
not introducing a set of referents that are limited to a frame. The signer is just making a list, which in (197) is a list of propositions.

$\frac{\text { re }}{\text { LIST1-IX-a MARINA CAKE CL:BAKE LIST2-IX-b JORDI PIZZA }}$

sp.ipsi.

CL:BAKE. (81.08)

'Marina baked a bake and Jordi baked a pizza.'

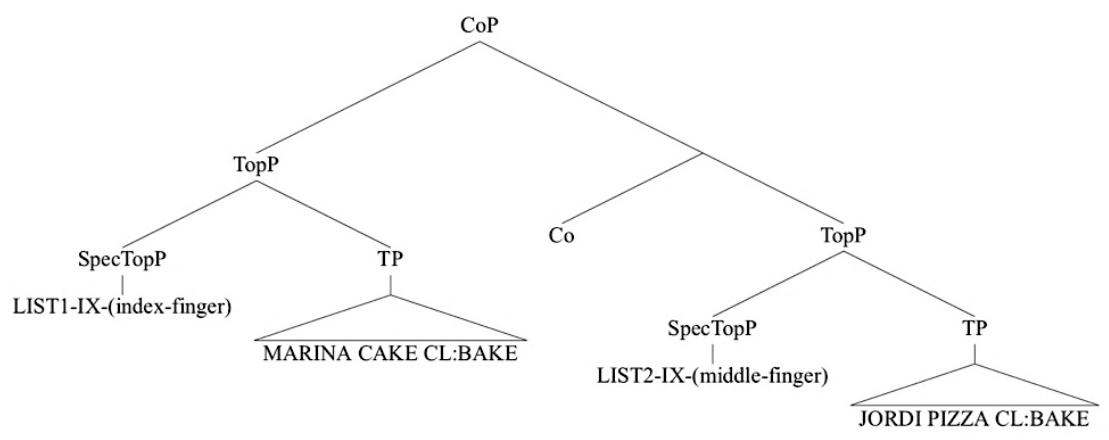

Concluding, list-buoys in LSC are used to list a set of referents or to arrange them in temporal order, whether they are propositions or individuals. They can appear in conjunctive or disjunctive coordination, but not in adversative coordination with counterexpectational BUT where a delimited set of referents holding to a certain frame is not presented. Depending on their function, indicated by the handshape and NMM, they can be represented as pronouns or adverbs. As for the total number of referents, due to the fact that the signer already knows how many s/he will present in the sentence, the frame setting that represents the non-dominant hand scopes over the whole coordination phrase in the specifier of $\mathrm{CP}$ 
placed above CoP. It scopes overtly when it is produced before introducing the conjuncts or covertly when it is not. When the weak hand is held though the whole sentence, the frame setting gets externally remerged in the specifier of each conjunct creating a multidominant structure; if it is not, frame setting is just merged on top of CP.

After presenting different aspects related to conjunctive coordination, and also to disjunctive one with list-buoys, we will now extend this analysis to disjunctive coordination.

\subsubsection{Disjunction (vs conjunction)}

In 3.1.2 we saw how disjunction can be expressed in LSC and noticed that it is mainly expressed asyndetically, as it is also the case for conjunction. When no overt markers are used, conjunction and disjunction can be distinguished only through the NMMs and the context. As in ASL, in LSC the conjuncts are marked using body and head lean or shift in combination with the use of space. No overt coordinator is usually produced between the two, though. Differently than what Davidson (2013) proposed, I am not considering LISTBUOY (Davidson's COORD-L) as a coordinator, but rather as a pronoun used to introduce the individuals or propositions contained in each conjunct, as we saw in the previous section 3.2. The other marker that she considers is COORD-SHIFT, which for LSC I have not considered a coordinator properly speaking, since it consists of only some of the NMMs that are used. I will not account for it, then, in the structure, where, for both conjunction and disjunction, the head of $\mathrm{CoP}$ is left 
empty. The presence of disjunction or conjunction will, instead, be marked by a quantifier scoping over the whole structure, as suggested by Davidson (2013). While in Davidson (2013) the structure used for coordination is XP, for LSC I will adopt CoP in the same way I did for conjunction in 3.2 above.

In 3.1.2 we saw examples with disjunction like the one in (199), where no overt manual markers are used and the disjunctive reading is determined by NMMs, as it is also the case for conjunction in (200).

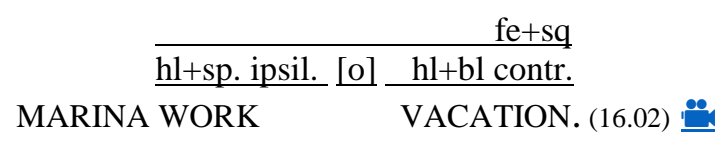

'Marina will work or she'll go on vacation.'

\begin{tabular}{ll} 
& \multicolumn{2}{c}{$\frac{\text { sp. contr. }}{\text { bl+hl contr. }}$} & bl+hl ipsil. \\
\cline { 2 - 3 } (200) & MARINA WORK VACATION. (83.14) \\
'Marina will work, and she'll go on vacation.'
\end{tabular}

Following Davidson (2013), it is possible to assume also for LSC that the coordinator, even if not overtly expressed, puts together two sets of alternatives (a set of more than one individual or alternative, e.g., $\{c, t\})$ and the conjunctive or disjunctive reading depends on the nature of the quantifier that scopes over the whole structure (in the case of LSC, CoP). In (201) we can see the semantic representation of the contribution of the quantifiers in disjunction (201a), with an existential quantifier, and conjunction (201b), with a universal one, both repeated from (113) in 2.3.4. In (202), instead, we can see the 
syntactic representation of sentence (199) for disjunction in LSC. For conjunction, instead, we assume a universal quantifier scoping over CoP instead of the existential one.
a. $\exists P=\lambda w \cdot \exists p[p \in P \wedge p(w)]$
(Alonso-Ovalle 2006)
b. $\forall P=\lambda w \cdot \forall p[p \in P \rightarrow p(w)]$

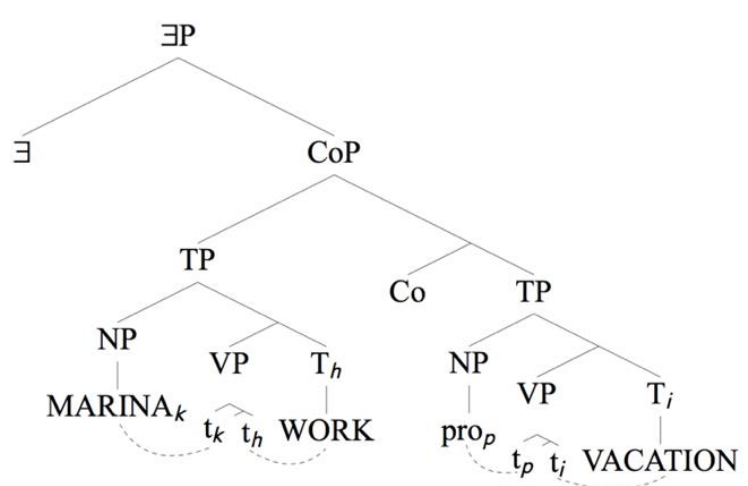

(LSC)

The presence of an existential or universal quantifier can be assumed in all cases of coordination expressing either conjunction or disjunction. Another case of possible ambiguity between the two types of coordination arises when a list-buoy is used and again only NMMs and context help to distinguish them. The context and other lexical elements in the sentence will in principle disambiguate the sentence anyway.

In 2.2.5.1 we saw other properties of disjunctive coordination that apply to English, like the change of scope of scope-operators such as either (Larson 1985). We saw that Larson (1985) proposes that when an element like either is displaced, then it takes clausal scope, differently from when it is in its base position. In LSC, though, 
such properties are not present due to the fact that the most productive position for the sign for either, EITHER-ONE, is at the end of the sentence and its displacement does not affect the meaning of the sentence. An exclusive reading can be conveyed using several other signs, as we saw in 2.2.5.1, but they are all preferably found at the end of the sentence. I shall not enter into the discussion on the interaction of these particles with the other elements in the sentence, considering their interaction with focused elements, and I will leave it for future research.

\subsubsection{Adversative coordination}

In this section we will present an analysis for adversative coordination in LSC based on Vicente's (2010) analysis for English and Spanish. As we saw for disjunction, this type of coordination will also show a structure in which the conjuncts are specifier and complement of CoP.

In 3.1.3 we saw that LSC shows corrective and counterexpectational adversative coordination, too. However, while in English and other languages like Spanish both types of coordinator but are always overt, in LSC corrective BUT can be omitted. In (203) we can see examples for each type: (203a, b) show corrective adversative coordination and (203c), counterexpectational one. 
space contr. re $\mathrm{hs}$ space ipsil. bl ipsil.

(203) a. MARINA ROOM STUDY NOT IX-LOC GARDEN PLAY. (78.05) 'Marina is not studying in her room, she's playing in the garden.'

hl ips. hl ips.

re re

b. MARINA APPLE THREE EAT BUT BANANA ONE. (81.02) 'Marina ate three apples but one banana.' re

bl forward

c. JORDI VERY-TALL BUT BASKETBALL PLAY VERY-BAD. (78.07) 'Jordi is very tall but he's very bad at playing basketball.'

The two examples in (203a) and (203b) can be classified under the same category as adversative coordination but, as we pointed out in 2.2.5.2, they need a different account. I will follow Vicente's (2010) "syntax of adversative coordination" and I will show that: i) corrective adversative coordination consists of clausal coordination plus deletion due to the presence of an elliptical site after the elements left in the conjunct move to the left periphery for information structure reasons (203b);

ii) counterexpectational but allows its conjuncts to be smaller than clauses.

(203a, b) figures as clausal coordination where the subject of the second conjunct is coreferential with the subject of the first one. (203c), instead, involves coordination of smaller constituents. This distinction can be seen applying several tests. For corrective but it is possible to look at preverbal subject coordination and at the scope of negation. Due to language specific properties of LSC, though, this 
last test cannot be used in counterexpectational adversative coordination. The presence or absence of deletion can be detected in both types, though, by looking at island sensitivity (Merchant 2001). I will adapt to LSC the tests presented in 2.2.5.2 from Vicente (2010) in order to show the different derivations for $(203 \mathrm{~b}, \mathrm{c})$. I will not enter the details of the analysis for (203a), but I would like to point out that even if corrective BUT is not overtly used, that is counterbalanced by a contrastive use in space of the two conjuncts. Such strategy of contrast in the use of space has been already marked in LSC by Barberà (2012).

Let us look first at the first test: preverbal subject coordination, in which two NPs are coordinated. If we assume the presence of clausal coordination for corrective BUT, we expect preverbal subject coordination to be ungrammatical. For counterexpectational BUT, instead, it should be good thanks to the option of coordinating conjuncts smaller than clauses. Let us examine the examples in (204). In (204a) we can clearly see that it is not possible to have preverbal subject coordination, while it is possible only in a regular corrective adversative coordination structure like the one in (204a'), where the polarity of the two conjuncts is clearly marked. In the case of counterexpectational BUT instead, it is good as expected (204b).

(204) a. *[TWO PERSON MATH BUT SEVEN PHYSICIAN] ARTICLE PUBLISH NOT. 
a'. [TWO PERSON MATH ARTICLE PUBLISH NOT] [BUT SEVEN PHYSICIAN ARTICLE PUBLISH YES]. (82.06)

‘*Two mathematicians but seven astrophysicists didn't publish their paper.'

b. [ASTRONAUT ONE THAT'S-IT NOT FOUR PEOPLE MINIMUM MATHEMATICIAN] 4-PARTICIPATE CAN. (82.07)

'Not one astronaut only but at least four mathematicians can participate.'

This difference can be clearly seen also with respect to island sensitivity. Merchant (2001) uses the impossibility of violating islands as a test to show the presence of structure in the ellipsis site, also proving the movement of the element produced in the second conjunct to its left periphery. In (205a) it is possible to see how the island sensitivity and therefore the presence of structure in the elliptical site is detected when using corrective adversative coordination. With counterexpectational BUT, instead, it is not the case due to the fact that there is no coordination of clauses, as in (205b).

(205) a. *[YESTERDAY PARTY IX-1 LEAVE [MARINA EXPLAIN JOKE AFTER] NOT] [[IX-3- POSS LIFE]i PARTY IX 1 LEAVE [MARINA EXPLAIN EXPLAIN ti AFTER]]. '??Yesterday I didn't leave the party after Marina explained jokes but her life.' 
b. [YESTERDAY CONFERENCE IX-1 LEAVE [BECAUSE [NP INTERPRETER ONE PERSON PROFESSIONAL] BUT [NP FOUR INTERPRETER INTERNS]]]. HORRIBLE PALM-UP. (83.03)

'Yesterday I left the conference because there was one professional interpreter but four interns. It was horrible.'

The same conclusion about corrective BUT could be reached on the basis of the scope of negation. In (206a) we can clearly see that the scope of negation in corrective adversative coordination is overtly marked as narrow also thanks to the overt marker YES that underlines the positive polarity of the second conjunct. In line with Vicente (2010) we can then confirm a narrow scope negation in (206a) that only affects the first conjunct and not the second one: $[(\neg p) \wedge q]$. A structure like the one in (206b) to represent (206a) is legitimated. I will not enter in the details concerning the nature or the position of YES, and shall leave this for further research.

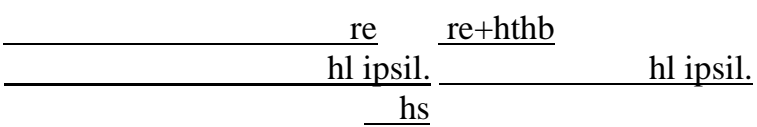

(206) a. JORDI BEER CL:DRINK NOT BUT SANGRIA YES. (81.04) 'Jordi didn't drink beer but sangria.'

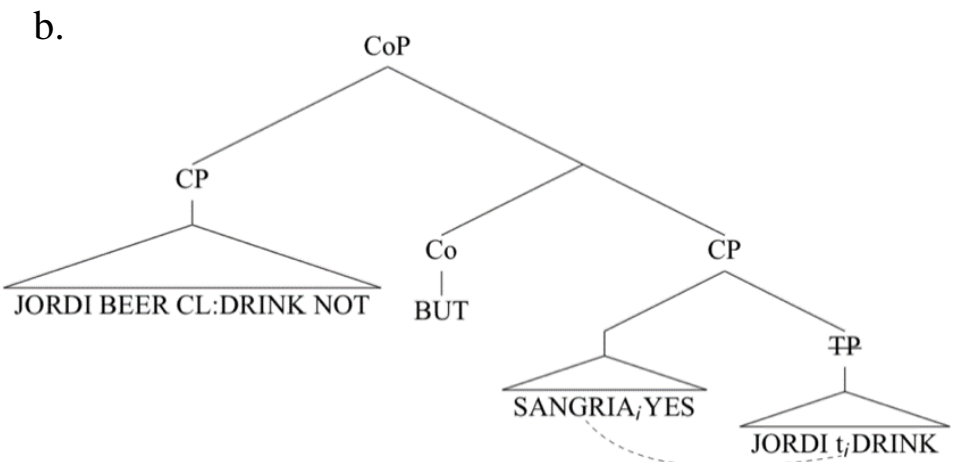


The structure in (206b) also supports the analysis for the direction of coordination we saw in 3.2.2. Having a right-branching coordination structure where the first conjunct is higher than the second one licenses the deletion of the material in the second conjunct. We will see further data on this aspect in chapter 5 when looking at gapping across languages. As for the scope of negation with counterexpectational BUT, it is not so straightforward to provide an analysis using data like (207). Therefore, I will not consider the scope of negation to prove the presence of subclausal coordination in this type of structures. Since negation is placed in the right periphery of the sentence and structurally it is found in a negative phrase (NegP) above TP (Pfau \& Quer 2007). No instances of ellipsis are anyway formed.

(207) MARINA POOR NOT BUT HUMBLE. (82.05) 를

'Marina is not poor but humble.'

We can conclude that also in LSC it is possible to identify different derivations for adversative coordination depending on whether corrective or counterexpectational BUT is used. Following Vicente (2010) we can confirm that coordination with counterexpectational BUT does not involve clausal coordination while it can be the case with corrective BUT. When corrective BUT is used in full clause coordination, no special derivation other than using a right-branching coordination structure is needed: the conjuncts are the specifier and the complement of CoP. In the examples above we also saw a common use of the polarity particle YES, but I shall leave for further 
studies its nature and position in the structure. For more research on but in spoken languages used in elliptical structures in relation with negation, see Repp (2009).

\subsection{Remarks on directionality of coordination and other SLs}

The data presented for coordination in LSC in this chapter raised a problem that has never been undertaken before for SLs, namely focusing on the directionality of coordination. For LSC we concluded that, despite LSC being a head-final language, it needs a rightbranching coordination structure. We will delve into this aspect also in 5.6 for other spoken languages like Farsi and we will present data on the directionality of gapping. It is the position with respect to the conjuncts that determines the directionality of the coordination structure. The same conclusion can be drawn also for SLs, even though for the moment we have data only for FinSL. In FinSL, Jantunen (2016) notices that an eye blink produced before the coordinator makes clear that in FinSL the coordinator belongs to the second conjunct, realizing prepositive coordination. As reported by Jantunen (2008) and Ala-Sippola (2012), in declarative transitive clauses FinSL shows two main word orders, regarding the position of A (agentive-like argument) and P (patient-like argument) with respect to the verb (V): AVP and APV. These two orders have been found in signing isolated clauses. In discourse settings, though, the order PAV seems to be highly marked (Ala-Sippola 2012). FinSL, then, shows both SOV and SVO order, where the latter is the 
predominant one. According to the description made till now regarding the directionality of coordination, from the fact that FinSL shows prepositive coordination and has predominantly SVO order follows from the need of having a right-branching coordination structure. Due to the position of the coordinator, the coordination structure can keep the same orientation also when it shows SOV order, as we saw for LSC. It would be interesting to have more data from other SLs in order to be able to extend this generalization. We will see in 5.6 that the position of the coordinator also affects orientation of gapping supporting the presence of (forward) gapping or RNR.

\subsection{Summary and conclusions}

In this chapter we described how coordination is expressed in LSC going through the strategies used to produce conjunctive, disjunctive and adversative coordination and showed that LSC is mainly expressed asyndetically, even in adversative coordination, which in spoken languages mostly features an overt but. Going through the syntactic properties of coordination in LSC we showed that it is possible to coordinate different syntactic categories, if they are both selected by the verb. No cases of precedence of one conjunct over the other is found, therefore weakening Zhang's (2010) analysis for coordination where the phrase of the structure is the same of the first conjunct that is always selected by the verb, contrary to what happens with the second one. The phrase used for coordination in LSC, then, is argued to be CoP, covering all three main types of coordination. 
Since no conjunct shows a special status, I find it important to have a category that indicates the relation among them. Moreover, the presence of coordination is identified from the beginning of the sentence or before the production of the coordinator thanks to specific NMMs. In addition, syntactic asymmetries were found also when semantic symmetries were present, supporting a default asymmetric syntactic structure for coordination in LSC. As for the directionality of the structure, assuming the conjuncts as being specifier and complement of $\mathrm{CoP}$, the position of the coordinator with respect to the conjuncts is crucial in determining the presence of a right or left branching coordination structure. I followed Zhang's (2010) generalization that languages with prepositive coordination have a right-branching coordination structure, while those with postpositive have a left-branching one, independently of the language being head-final or head-initial. LSC turns out to need a rightbranching coordination structure. LSC features prepositive coordination according to intonation grouping and extraposition. Moreover, applying the tests proposed in the literature to identify coordination in SLs (the limitations in applying ATB movement in wh-questions or having negation scoping over both conjuncts as well as not performing RNR) confirm the presence of a right-branching coordination structure in which the second conjunct is lower than the first one. More data will be presented to support this analysis in chapter 5 when discussing the directionality of gapping crosslinguistically.

Focusing now on the analysis, by adopting Munn (1987), in which the conjuncts are specifier and complement of $\mathrm{CoP}$, we 
discussed a syntactic proposal that can account for conjunctive, disjunctive and adversative coordination. For each type it was necessary to add details to the derivation of the structure. In the case of simultaneous events produced through weak hand holds or simultaneous use of both hands we adopted a multidominant structure that was extended also to the analysis of list-buoys, partly following Kimmelman (2017). For disjunctive coordination, instead, due to the similarity in the production of conjunction and disjunction, we adapted to LSC Davidson's (2013) semantic quantificational analysis, which assumes the presence of a universal quantifier for conjunction and an existential one for disjunction scoping over the conjuncts. Finally, for adversative coordination, we considered different derivations depending on the type of structure in which corrective BUT was found, either full clause coordination or an elliptic structure, or structures with a counterexpectational BUT. In corrective adversative coordination in ellipsis, following Vicente (2010), in LSC it is possible to confirm the presence of clausal coordination plus deletion. When there is correction expressed in full clausal coordination without an over BUT used, instead, bigger constituents are involved, and no ellipsis is found. We also saw cases of correction where the polarity of the two conjuncts is overtly expressed with negation in the first conjunct and the sign YES in the second one. More research is needed to evaluate the nature of this polarity element. Finally, for counterexpectational adversative coordination, subclausal coordination and no instances of ellipsis are found.

The study of coordination in LSC shed light on several aspects that had not been analyzed before. The directionality of 
coordination helps to link some of the data that seem to make coordination in LSC special. As we saw, ATB movement and scope of negation cannot be used as proper tests to identify coordination. This is due to the fact that most of the tests have been proposed for head-initial languages. LSC is a head-final language showing prepositive coordination and this looks like a good argument to justify apparent anomalies in coordination. Wh-elements and negation appear on the right periphery of the sentence; therefore they will be found at the end of a non-final conjunct when trying to make them scope over the whole structure. As we saw, this is not possible and it can be justified by the fact that the second conjunct is lower than the first one in a right-branching coordination structure. This data matches also the presence of (forward) gapping and the impossibility of having RNR. In chapter 5 we will present further data to extend this generalization to justify the presence of gapping and RNR in head-initial and final languages. No specific study in LSC has been undertaken on ATB movement or scope of negation but this data is an interesting point to start with, also because, in LSC, they show a different behavior in comparison to languages like English. This data is also important to show the problems of adopting a multidominant structure to analyze ATB movement, even though multidominance cannot be excluded to account for other phenomena such as production of simultaneous events in LSC. On this point, it would be interesting to provide an analysis of the phonological and articulatory restrictions in the production of simultaneous events through weak hand holds and the use of both hands keeping the movement of the two signs. The rare use of the latter way of 
expressing simultaneous events is likely to be due to those restrictions, but no formalization has been done yet.

In the analysis of coordination provided in this chapter we focused on proposing a structure that could account for the behavior and the position of the coordinators used to express conjunction, disjunction and adversative coordination. For the first two types, the fact that their distinction is based on NMMs and context, without resorting to extra lexical elements, can be accounted by the presence of a quantifier that is scoping over the conjuncts as set of alternatives. Adversative coordination, as well, though, is not necessarily expressed overtly. It would then be interesting to implement the analysis of this type of structure, too, by looking at the interaction with information structure in the line with what Repp (2010) studied in gapping structures. In chapter 5 we will give an analysis of gapping in conjunction in LSC, but we will not go through data involving adversative coordination. I shall leave such analysis for the future. 
3. Coordination in Catalan Sign Language (LSC) 
210 


\section{PART II: GAPPING}


212 


\section{ELLIPSIS AND GAPPING}

In this chapter we will present the background for ellipsis, focusing in particular on gapping in conjunction, the elliptical structure we will look at in chapter 5 for LSC. We will first give a general introduction to the phenomenon of ellipsis in 4.1, where we present the main approaches proposed and also explore one of the main questions in ellipsis that concerns the nature of the relationship between the elliptical site and its antecedent. In 4.2 we will focus on gapping and we will also review its properties and the main approaches that have been proposed in the literature. Finally, data on gapping in SLs will be reported. In this chapter we will mainly focus on English due to the large amount of work that has been done on this language in the domain of ellipsis.

\subsection{Introduction to ellipsis}

Ellipsis refers to cases in which expected material goes missing under certain conditions, excluding cases of implicit arguments (van Craenenbroeck \& Merchant 2013; Merchant 2010). The ellipsis site can target sentential, predicate or nominal material, realizing meaning without form. In the literature, one of the main puzzles concerns the recoverability of the material that is missing, leading to two central questions: i) is there unpronounced syntactic structure in the ellipsis site? and ii) is the identity between the ellipsis site and its antecedent syntactic or semantic? Merchant (2012) schematically 
puts together the different approaches that answer these two questions, as we can see in Table 1.

\begin{tabular}{|c|c|c|c|}
\hline & \multicolumn{2}{|c|}{ Is there syntax in the ellipsis site? } \\
\hline & & Yes & No \\
\hline \multirow{2}{*}{ 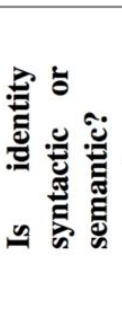 } & Syntactic & $\begin{array}{l}\text { Sag 1976, Williams 1977, } \\
\text { Fiengo \& May 1994, } \\
\text { Chung et al. 1995, } \\
\text { Kehler 2002, etc. }\end{array}$ & N/A (incoherent) \\
\hline & Semantic & $\begin{array}{l}\text { Sag and Hankamer 1984, } \\
\text { Merchant 2001, } \\
\text { van Craenenbroeck 2010, } \\
\text { Aelbrecht 2010, etc. }\end{array}$ & $\begin{array}{c}\text { Keenan 1971, Hardt 1993, } \\
\text { Dalrymple et al. 1991, } \\
\text { Ginzburg \& Sag 2000, } \\
\text { Culicover \& Jackendoff 2005, etc. }\end{array}$ \\
\hline
\end{tabular}

Table 1: Different approaches that face the two main ellipsis questions (Merchant 2012).

As for the material missing, it is possible to identify different types of ellipsis and distinguish clausal ellipsis, predicate ellipsis, and nominal ellipsis. In (208) we can see examples of clausal ellipsis in English: sluicing, gapping, stripping, and fragment answers, in (208a-d) respectively. (208e) and (208f) are, instead, cases of verb phrase (VP) ellipsis and pseudogapping, two cases of predicate ellipsis. Finally, in (208g), there is an example of nominal ellipsis (NP ellipsis). In this chapter we will focus only on the first two main types, clausal and predicate ellipsis, without focusing on nominal ellipsis (exemplified in (208g)).

(208) a. Ed killed someone, but I don't know who. (Sluicing)

b. John likes sandals and Mary stiletto heels.

(Gapping) 
c. Ed likes stiletto heels and Maggy, too.

(Stripping)

d. A: What did you buy? B: A boat.

(Fragment answers)

e. John likes candy, but Bill doesn't

(Verb Phrase Ellipsis)

f. She'll read something to Sam, but she won't to Bill.

(Pseudogapping)

(van Craenenbroeck and Merchant 2013: 702, 718)

g. Abby can play five instruments, and Ben can play six. (NP ellipsis)

(Merchant 2012: 02)

A fairly recent general tendency in analyzing ellipsis assumes the presence of deletion of material. The portion of structure affected depends on the type of structure and on the properties that are taken into account. Information structure, for instance, is an important factor in determining the derivation of ellipsis. In VPE ellipsis, for example, it is assumed that the subject in each conjunct is focalized, and therefore moved to FocP (Frazier et al. 2013, among others). Not all analyses assume the presence of syntactic structure in the elliptical site, though, as we saw in Table 1. Let us first look at the different approaches proposed (4.1.1) and at their consequences in determining the relation between the ellipsis site and its antecedent (4.1.2) 


\subsubsection{Analysis: different approaches}

As mentioned above in Table 1, it is possible to distinguish between two main types of approaches to ellipsis: non-structural and structural. The former assume that there is no syntactic structure in the ellipsis site, while the latter do. These last ones can be further distinguished as to whether the unpronounced structure is there during the whole derivation or not. If it is, ellipsis is analyzed as deletion at PF; otherwise, it is assumed to contain an LF-copy or presence of null anaphora. Let us look next at these approaches in more detail.

\subsubsection{Non-structural approaches}

Non-structural approaches follow the idea of "what you see is what you get", namely that there is no structure in the ellipsis site, there is neither deleted material nor null elements. Ginzburg and Sag (2000), Schlangen (2003), Jacobson (2003), Culicover \& Jackendoff (2005), among others, follow this line. In a case of sluicing, for example, the verb know in (209a) is supposed to take the wh-phrase as its complement, which is the only material present as daughter of the $S$ node complement. Merchant (2001: 40-54), though, points out that the sluiced phrase is a clausal complement and not a nominal one. Moreover, in (209b) it is possible to see how the extracted whelement is not selected by the verb know but rather by the verb in the ellipsis site (we will come back to other tests to show the presence of material in the ellipsis site in the next section 4.1.1.2). 
(209) a. Someone had brought strawberries, but I don't know [s who].

b. I know which puppy YOU should take home, but I don't know which ones SHE should [take home $t_{\text {which ones]. }}$

(Aelbrecht 2015: 571, 578)

An implementation of this approach is given by Culicover and Jackendoff (2005), who propose a Simpler Syntax Hypothesis in which "the most explanatory theory is one that imputes the minimum syntactic structure necessary to mediate between phonology and meaning" (Culicover \& Jackendoff 2005: 5). In their account they claim that in the case of sluicing, for example, the verb selects an $\mathrm{S}$ node that is marked for "indirect licensing" (IL) and the wh-element in it is considered an "orphan" (ORPH). As we can see in (210), the orphan NP, even though it is realized as a wh-phrase, receives its semantic and syntactic features from the antecedent. The semantics is given by a free variable $\mathrm{F}$ whose value is given by the context through indirect licensing. The wh-word $\left(\mathrm{x}_{\mathrm{i}}\right)$, in fact, is bound by a question operator Qx.

(210) Syntax: $\left[\mathrm{s} \text { wh-phrase }{ }_{i}{ }^{\mathrm{ORPH}}\right]^{\mathrm{IL}}$

Semantics: Qx [F( $\left.\left.\mathrm{x}_{\mathrm{i}}\right)\right]$

(Culicover \& Jackendoff 2005: 270)

As we will see in the next section, though, there are several arguments that point to a structural analysis of ellipsis. 


\subsubsection{Structural approaches}

Among the structural approaches, which all assume the presence of structure in the ellipsis site, it is possible to identify different assumptions on the nature of the unpronounced structure: either it is made of null elements or the material that is present gets deleted.

In the first case, it is possible to have a null proform approach or an LF-copy analysis. In the null proform approach, the ellipsis site is considered to contain a null proform pro that is interpreted like an overt pronoun (Hardt 1993; Lobeck 1995). In the LF-copy analysis, instead, the antecedent is assumed to be copied into the ellipsis site at LF and it posits null elements in the syntax (Wasow 1972; Fiengo \& May 1994; Chung et al. 1995; Wilder 1997; Beavers \& Sag 2004, Fortin 2007). Independently of how the null elements are derived, the example we saw in (209a) would be represented as in (211), in which the proform pro stands for the IP.

(211) Someone had brought strawberries, but I don't know [CP who [C pro $\left.\left._{I P}\right]\right]$.

In the deletion approach, instead, the syntax in the ellipsis site is considered to be the same of its antecedent, but it gets deleted thanks to a feature that indicates that the phonological value of the constituent targeted is null (e.g., VP in VP ellipsis).

Following Merchant (2001), van Craenenbroeck (2010), Aelbrecht (2009), van Craenenbroeck \& Lipták (2006), Toosarvandani (2008), Toosarvandani (2009), Vicente (2006), 
Corver \& van Koppen (2010, 2011), and Ha (2008), this feature is called E-feature, standing for Ellipsis-feature. It has a syntax, a semantic and a phonology, since it keeps all the information about the ellipsis site. In Merchant (2001), this feature is added to the "licensing" head indicating that all the material present in its sister gets deleted. In the case of sluicing, the structure would be the one in (212). For sluicing the E-feature is, then, placed in the head of CP indicating the deletion of the whole TP.

(212) Someone murdered Joe, but we don't know [who $]_{1}<t_{1}$ murdered Joe $>$.

(Merchant 2012: 21)

There are several pieces of evidence that support the existence of structure in the ellipsis site: island sensitivity, case matching effects, complementizer deletion, distribution of infinitivals, predicate answers and intermediate reconstruction effect in sluicing. In this chapter we are going to only present data on island sensitivity that will be used also for LSC in chapter 5. The other phenomena cannot be tested in SLs due to the absence of morphological case, prepositions, overt complementizers like "that" and infinitivals.

Locality effects shown in island sensitivity is used in different types of ellipsis, as we can see in (213a) for VPE, in (213b) for fragment answers and in (213c) for gapping. In VP ellipsis, fragment answers and gapping and other structures, it is not possible to extract out of an island. If no structure were present, extraction would have been allowed. 
(213) a. *I read every book you introduced me to a guy who did.

b. A: Did each candidate 2 agree on who will ask him about taxes (at tonight's debate)?

B: *No, about foreign policy.

C: No, each candidate 2 agreed on who will ask him2 about foreign policy (at tonight's debate)

(Merchant 2012: 09, 10)

c. *Suzy doesn’t like men who play instruments, and Mary, sports.

$(\text { Coppock 2001: 12) })^{21}$

Culicover \& Jackendoff (2005: 273), though, point out examples that show that island violation is possible in gapping, as we can see in (214). They underline the same also for cases of fragment answers, as in (215) (op. cit.: 244ff).

(214) Robin knows a lot of reasons why dogs are good pets, and Leslie, cats.

(215) A: John met a woman who speaks French.

a. B: And Bengali?

b. *And Bengali, did John meet a woman who speaks French $t$ ?

Merchant (2012), though, lists all the possible readings for (215a) and none seems to show island violation. Moreover, the readings in (216)

\footnotetext{
${ }^{21}$ The first data on island sensitivity in gapping were reported by Levin (1986) but here I will use Coppock's (2001) examples.
} 
can be accounted for in a structural approach, but not in a nonstructural one.

(216) a. = Did John meet a woman who speaks French and Bengali?

b. $=$ Does she speak French and Bengali?

c. $=$ And does she speak Bengali (too)?

d. = And what about Bengali?

e. $=$ And how about Bengali?

f. $\neq$ And did John also meet a different woman who speaks

Bengali (in addition to meeting the woman who speaks French)?

(Merchant 2012: 16)

Other arguments have been used to support the non-structural approach, mainly the possibility that ellipsis can take a non-linguistic antecedent, like in (217), and the so called "sloppy ellipsis" puzzle: in a sentence like (218) it is possible to have a sloppy and a strict reading of the verb in the second conjunct of an elliptical structure, but that is not the case when the VPs are deaccented (Hardt 1999; Schwarz 2000). The strict reading in (218a) and the sloppy one in (218b) are acceptable. The material that would give a sloppy reading, though, if pronounced in the second conjunct, would give an ungrammatical sentence, like in (218c). In this latter case, no sloppy reading is available (218c). For an extensive discussion of these facts and an analysis in favor of the presence of syntactic structure, see Merchant (2012: 22-27). 
(217) You shouldn't have! (On receiving a present)

(Lobeck 1995)

(218) I'll help you if you want me to. I'll kiss you even if you don't $<>$.
a. $\langle>=<$ want me to help you $>($ STRICT)
b. $\langle>=<$ want me to kiss you $>$ (SLOPPY)
c. $\langle>\neq<$ want me to kiss you $>(*$ SLOPPY)

Despite the presence of counterarguments to the structural approach, it seems to be the most consistent, especially because it assumes the same syntax in both the antecedent and the elliptical site where the E-feature indicates what is missing.

The question related to the nature of the relation between the antecedent and the elliptical site usually comes in two flavors. It has been proposed that between the two there is syntactic identity or semantic identity, even though others have come to the conclusion that both are present. We will see next the debate concerning this aspect.

\subsubsection{Identity}

As we presented in section 4.1, an important question that is addressed in relation to ellipsis is the nature of the relation between the antecedent and the material in the ellipsis site, if there is any. In Table 1 we saw a summary of the different approaches proposed. In the next two sections we will go through the arguments that support the presence of syntactic or semantic identity. 


\subsubsection{Syntactic identity}

There are two main arguments that support the presence of syntactic identity: syntactic and morphological mismatches.

As for the first type, the distribution of voice mismatch in different types of ellipsis can be considered a strong argument. As we can see in (219) with a sluicing example, there must be match in voice in types of ellipsis that Merchant (2012) defines as "big/high", including also fragments answers, gapping and stripping. In "low/little" ellipsis, instead, like in VPE in (220), voice mismatch is allowed.

(219) a. passive antecedent, active ellipsis (sluicing):

*Joe was murdered, but we don't know who. $<$ murdered Joe>

b. active antecedent, passive ellipsis (sluicing):

*Someone murdered Joe, but we don't know who by. $<$ Joe was murdered>

(220) a. passive antecedent, active ellipsis (VPE):

This problem was to have been looked into, but obviously nobody did. <look into this problem>

b. active antecedent, passive ellipsis (VPE):

The janitor should remove the trash whenever it is apparent that it needs to be <removed $>$.

(Merchant 2012: 30-31) 
The explanation of this data given by Merchant $(2012,2013 b)$ is derived from the position of voice, following Rivero (1990) and Kratzer (1996), who assume the voice head to be separated from the rest of the VP and be placed in a projection higher than VP. In "high" ellipsis, then, voice cannot be interpreted in the second conjunct because it would get deleted along with the whole CP/TP. In cases of VPE, instead, it is the vP that gets deleted, but not the voice head.

Other data presented by Warner (1985) and discussed by Lasnik (1995) and Potsdam (1997) show that verbs do not require morphological identity, as in (221), even though it is not the case for forms of be, which do require it, as in (222). (221) and (222) are the illustrations by Merchant (2012). In (221) we can see that, even though the structure in the ellipsis site does not correspond to the antecedent, in (221a) it is totally recoverable, with the interpretation in (221b). In (222a), instead, this is not possible, with the intended interpretation conveyed in (222b).

(221) a. Emily played beautifully at the recital and her sister will too.

b. Emily played beautifully at the recital and her sister will play beautifully at the recital, too.

(222) a. *Emily was beautiful at the recital and her sister will _, too.

b. Emily was beautiful at the recital and her sister will be beautiful at the recital, too. 
Lasnik (1995) accounts for this data considering that the forms of be enter the derivation fully inflected, while, for the other verbs, the syntactic identity is met before inflection. It is possible to get to a similar conclusion also for data where there is categorial mismatch, like in (223), from Hardt (1993).

(223) David Begelman is a great [laughter], and when he does [łaugh], his eyes crinkle at you the way Lady Brett's did in The Sun Also Rises.

The syntactic identity is therefore assumed to be established in the derivation and not necessarily at the end of it, in order to account for these surface mismatches.

Fiengo \& May (1994) also support the identity in form between the antecedent and the ellipsis site by presenting data of a phenomenon called "vehicle change", in particular considering pronoun/name equivalence, previously discussed also by Dalrymple (1991). In (224) we can see that the sentence is ungrammatical because there is a violation of principle $\mathrm{C}$ : the referential expression John in the second conjunct is c-commanded by a coindexed pronoun (224b). In (225), instead, the sentence is grammatical because John can undergo vehicle change, whereby a pronoun replaces the referential expression in the elided VP, but preserves its indexical information (Fiengo and May 1994).

(224) a. *Mary admires $\mathrm{John}_{\mathrm{i}}$ and he $\mathrm{e}_{\mathrm{i}}$ does, too.

b. *Mary admires John $n_{i}$ and he $\mathrm{i}_{\mathrm{i}}$ does admire John $\mathrm{H}_{\mathrm{i}}$ too. 
(225) a. Mary admires $\mathrm{John}_{\mathrm{i}}$, and he thinks that Sally does too.

b. Mary admires $\mathrm{John}_{\mathrm{i}}$, and he $\mathrm{i}_{\mathrm{i}}$ thinks that Sally does admire himmit $_{\mathrm{i}}$ too.

As Merchant (2012) points out, though, "it is difficult to see how he and John could be syntactically equivalent". For him, then, vehicle change is an argument for semantic identity.

As we will see in 4.2.3, data from SLs also support identity in form, even though further data on LSC in gapping to be discussed in chapter 5 will challenge this view.

\subsubsection{Semantic identity}

The two main views that support semantic identity are made by Hardt (1993) and by Merchant (2001). In the first case, it is assumed that in the ellipsis site there is no syntactic structure. The VP in the ellipsis site is considered an indefinite property parallel to a pronoun that selects its meaning from the discourse, as we can see in (226). Therefore, a cause of the absence of structure, the recoverability condition is due to semantic identity.

(226) John parked the car. Mary did pro, too.

For Merchant (2001), instead, there is structure in the ellipsis site, but the possibility of deleting material is given by the presence of eGIVENness (Rooth 1992, Schwarzchild 1999). As reported in Merchant (2001: 26), in order to license identity between the ellipsis 
site and the antecedent, it is necessary to respect the definitions in (227).

(227) (i) e-GIVENness Condition

A VP or IP $\alpha$ can be deleted only if $\alpha$ is e-GIVEN.

(ii) e-GIVENness

An expression E counts as e-GIVEN iff E has a salient antecedent $\mathrm{A}$ and, modulo $\exists$-type shifting,

i. A entails F-clo(E), and

ii. E entails F-clo(A)

(iii) F-closure

The F-closure of $\alpha$, written as F-clo $(\alpha)$, is the result of replacing F- marked

parts of $\alpha$ with $\exists$-bound variables of the appropriate type (modulo $\exists$-type shifting).

We can see an example of the definitions in (227) applied in (228), adapted from Merchant (2001). The subindex $A$ is marking the antecedent and $E$ the ellipsis site. Due to the fact that A and $\mathrm{E}$ entail each other, it is possible to have deletion, as in (228a). Looking at (228b), even though the antecedent entails that Kim insulted Pat, the entailment does not happen the other way around. Therefore, the only possible reading is the one in (228a) and not the one in (228b). The same approach is also followed by Coppock (2001) for gapping, as we will see in 4.2.2. 
(228) Alice called Pat an idiot after Kim did.

a. $=$...after Kim did eall Pat an idiot.

(i) F-clo $\left(\mathrm{VP}_{\mathrm{A}}\right)=\exists \mathrm{x} \cdot \mathrm{x}$ called Pat an idiot

(ii) F-clo $\left(\mathrm{VP}_{\mathrm{E}}\right)=\exists \mathrm{x} \cdot \mathrm{x}$ called Pat an idiot

b. $\neq$...after Kim did insult Pat.

Other authors, though, also proposed hybrid theories that combine both semantic and syntactic identity (see Kehler 2002, Chung 2006, van Craenenbroeck 2008 and Merchant 2013a).

After presenting an overview of the main questions related to the phenomenon of ellipsis in general, we are going to focus on gapping. The data presented will be the base to discuss the ones on LSC in chapter 5.

\subsection{Gapping}

In this section dedicated to gapping we will first go through the main properties that characterize this structure, and take into account its interaction with information structure. We will mainly focus on English, but also consider other languages like Farsi, among others. We will present the main analyses that have been proposed, as well, dividing them in two groups (the ones that propose a small-conjunct coordination and the ones that suggest a large-conjunct coordination), and we will further distinguish among those that consider the presence of sideward movement, ATB movement or deletion. Finally, before getting into the data on gapping in LSC in chapter 5, 
we will present the evidence reported till now for gapping and VPE in LIS, HKSL and FinSL.

\subsubsection{Properties of gapping}

Gapping is a type of ellipsis in which a verb is removed in one, or more, of a series of coordinations, as defined by Johnson (2014: 1). Moreover, the unpronounced material must include the verb, but other elements, such as arguments and adjuncts, can also be included. There are two crucial aspects: having identity between the gapped site and the antecedent and contrast between the remnants and their correlate antecedents in the previous conjunct. In (229) we can see an example of this structure that was first studied by Ross (1967).

(229) a. Some ate beans and others, rice.

b. Some talk about problems openly and others cautiously. (Johnson 2004: 1)

Ross (1970) studied the mechanism of gapping and got to the conclusion that there are two types of gapping: forward and backward. In the first type the verb is missing in the second conjunct, like in English in (229). Backward gapping, instead, is assumed by Ross to be found in languages like Japanese, where the verb is missing in the first conjunct, as we can see in (230). 
(230) Watakusi wa sakana o, Biru wa gohan (Japanese) I (prt) fish (prt), Bill (prt) rice o tabeta. (prt) ate

'I ate fish and Bill rice.'

Johnson (2014), among others, though, goes against Ross's generalization, in assuming that the verb can only be missing from the second conjunct in gapping. Otherwise, it is a case of Right Node Raising (RNR). In (231) we can see an example from Postal (1974) in which the object of the verb, shared by both conjuncts, is removed in the first one.

(231) Jerry met the kids from OshKosh and Sally scrutinized the kids from OshKosh.

In (232)-(235) we can see that gapping and RNR in English do not share the same properties and therefore can be distinguished, as suggested by Johnson (2014) contra Ross (1970), following Maling (1972), Neijt (1979), Hankamer (1979); more recently Citko (2018) suggests the same distinction for Polish. In (232) we can see that gapping, but not RNR, allows a mismatch in inflection between the gapping site and its antecedent. Moreover, in RNR it is possible to remove part of a word, but it is not the case for gapping (233). Also, while RNR needs the material to be elided in a continuous string, this is not necessary in gapping (234). Finally, RNR can strand prepositions, but gapping cannot (235). Maling (1972), Hankamer 
(1979) and Bozsahin (2000) also refer to cases in which the verb is missing in the first conjunct in Turkish as instances of RNR.

(232) a. He likes beans and you like rice.

b. *He always eomplains and you sometimes complain.

(233) a. *Carly is overpaid and Will underpaid.

b. Carly is overpaid and Will underpaid.

(234) a. Carrie gave a set of directions to me, and Will a map tome.

b. *Carrie gave a set of directions to me, and Will gave a map to me.

(Johnson 2014: 4)

(235) a. *John is confident of a successful outing and Peter is dependent on a successful outing.

b. John is confident of a successful outing and Peter is dependent on a successful outing.

(Neijt 1979: 40)

For it to be considered gapping, then, the gap can only appear in the second conjunct of two conjuncts, following Johnson (2014). This generalization holds for both head-initial and head-final languages. Ross (1970), in fact, claims that there is no language that can only gap forward having SOV as underlying order (*SOV-SO), but this is not the case for languages like Farsi (Carrera-Hernández 2007, Farudi 2013) and also Turkish (Bozsahin 2000), as we can see in (236) and (237), respectively. In chapter 5 we will see that LSC shares the same properties, too. 
(236) Rodmehr gusht xord va Anahita māhi.

Rodmehr meat ate.3sg and Anahita fish

'Rodmehr ate meat and Anahita fish.'

(Farudi 2013: 65)

(237) Adam kitabi okudu, çocuk da dergiyu.

(Turkish)

man book-acc read-past child conj mag-acc

'The man read the book, and the child, the magazine.'

(Bozsahin 2000: 6)

As for the material that is targeted, differently from other types of ellipsis, gapping always shows in the second conjunct an element belonging to the VP, while the verb is missing. The element left in the second conjunct needs to be in a contrastive relation with a similar element in the first one. We will see in more detail the implications of this relation, also with respect to information structure, in the following section (4.2.1.1).

Among the properties of gapping, one that seems to be common is the restriction for gapping to appear only in coordination (Jackendoff 1971, Hudson 1976, Johnson 2009), as illustrated in examples (238) and (239) from English and Farsi, respectively.

(238) a. Some had eaten mussels and others shrimp.

b. *Some had eaten mussels because others shrimp. (Johnson 2009: 1, 6)

(239) a. Rādmehr diruz gushtxord va Gitimāhi. (Farsi) Rodmehr yesterday meat ate.3SG and Giti fish 'Rodmehr ate meat (yesterday) and Giti (ate) fish (yesterday).' 
b. ?/??/*Ānāhitā māhi xord chunke Giti gusht Annahita fish ate.3sg because Giti meat INTENDED: 'Annahita ate fish because Giti (ate) meat.'

(Farudi 2013: 58, 64)

This, though, seems to be a special property of gapping, at least in English and Farsi, since other ellipsis structures like VPE can be found in subordination, as we can see in (240).

(240) a. John will have caviar, although others won't.

VPE

b. *John will have caviar, although others gapping beans.

c. John will have caviar, although others will pseudogapping beans.

d. John will have something, although I don't

sluicing know what.

In relation to this property, Hankamer (1979) states the No embedding constraint. In English, as exemplified in (241), the gapped verb cannot be embedded (cf. (241a)), and the antecedent of the gapped verb cannot be embedded either (cf. (241b), where the sentence is taken (somewhat awkwardly) to be a conjunction of two clauses and not a single one with an embedded coordination).

(241) a. *Alfonse stole the emeralds, and I think that Mugsy stole the pearls. 
b. *She's said Peter has eaten his peas, and Sally has eaten her green beans, so now we can have dessert.

(Hankamer 1979: 19,20)

In English, this constraint applies only to gapping and not to other types of ellipsis as VPE. Johnson (2014), then, uses this property as a diagnostic to identify gapping and he extends this generalization to all Germanic languages. In Farsi, though, for example, this diagnostic does not hold, while it does for Turkish (Ince 2009), Dutch (Aelbrecht 2009) and Hindi (Farudi 2013). Farudi (2013) shows that in Farsi the gap can appear in an embedded context, as we can see in (242). In 5.1 we will see that it is the case also for LSC. Moreover, in Farsi, the antecedent can be embedded as well, differently than English (243).

(242) Māmā chaī xord va fekr mi-kon-am (Farsi) mother tea ate-SG and think IMPFV-do-SG bābā qahve father coffee

'Mother drank tea and I think father coffee.' (Farudi 2013: 76)

(243) Fekr mi-kon-am ke Nasrin gormeh sabzi-ro (Farsi) think IMPFV-do-SG that Nasrin stew green-OBJ dorost kard va man adas polow-ro. make did.SG and I lentil rice-OBJ 'I think that Nasrin made spinach stew and I lentil rice.' 
In Farsi, as in English, if the gap or the antecedent are in a position where movement is blocked, the sentence is ungrammatical, as we can see in (244). Gapping, then, both in Farsi and English, is sensitive to islands. As we will see in 4.2.2, this a consistent test to show the presence of structure in the gapped site, as we saw in 4.1.1.2 for ellipsis in general.

(244) a. *Suzy doesn't like men who play instruments, and Mary, sports.

(Coppock 2001: 12)

b. *Giti mardhā-yi ke futbāl bāzi mi-kon-an

Giti men-IND that soccer play IMPFV-do-PL dust dār-e va Sārā mardhāyi ke tenis dust dār-e. like have-SG and Sara men who tennis like have-SG 'Giti likes men who play soccer and Sarah likes men who tennis.'

(Farudi 2013: 72)

Another property that will be used as argument in favor of a smallconjunct approach, as we will see in 4.2.2.1, is the presence of binding across conjuncts, or so called cross-conjunct binding (Lin 2000, 2002). As we can see in (245a), the subject of the first conjunct can bind the subject of the second one. When the verb is overtly produced in the second conjunct, the same binding relation is not allowed, as in (245b). Moreover, as pointed out by Johnson (2004), this relation is not allowed either in other types of ellipsis, like in VPE in (245c). 
(245) a. No one's duck was moist enough or his mussels tender enough.

b. *No one's duck was moist enough or/and his mussels were tender enough.

(McCawley 1993: 248)

c. *Not every girl 1 ate a green banana and her 1 mother did too.

(Johnson 2004: 26)

Finally, among the properties of gapping, it is important to also notice the effects of the scope of negation, modals and adverbs over the conjuncts. Oehrle (1987) and Siegel (1987), among others, noticed that these elements can scope over both conjuncts, therefore they can show wide scope $(\neg(A \wedge B))$, as we can see in (246).

(246) a. Kim didn't play bingo and Sandy didn't sit at home all evening.

(Oehrle1987: 205)

b. I tried it in both positions, one of which must have been the locked position and the other one the unlocked position, but it wouldn't work either way.

(McCawley 1993: 249)

c. Kim has often gone to the beach and Valerie to the city.

(modified from Oehrle1987)

It is assumed that in gapping, though, the scope of these elements is ambiguous between wide and distributed ${ }^{22}$ scope. In (247) and (248)

\footnotetext{
${ }^{22}$ I will be using "distributed" scope referring to the distribution of an element over each conjunct:
} 
we can see the paradigm presented by Siegel (1987), where a modal auxiliary, sentential negation and the main verb are involved. From this data it is possible to conclude that the presence of wide scope does not depend on the presence or absence of the main verb, but rather of the auxiliary. In the examples in (248), where gapping of both the auxiliary and the main verb is used, it is possible to have both readings: wide (248b) and distributed (248c) scope of the negation (see also Repp 2009: 106-110).

(247) a. John can't eat caviar and Mary can't eat beans.

Distributed scope $((\neg \mathrm{A}) \wedge(\neg \mathrm{B}))$

b. John can't eat caviar and Mary eat beans.

Wide scope $(\neg(\mathrm{A} \wedge \mathrm{B}))$

(248) a. John can't eat caviar and Mary, beans.

Ambiguity

b. $\neg$ [can [ John eat caviar and Mary eat beans ] ]

c. $\neg$ [can [ John eat caviar]] \& $\neg$ [ can [ Mary eat beans ]

(adapted from Siegel 1987, 7-9)

Winkler (2005) actually points out that wide scope reading in gapping needs a special context and it is realized only when denial is expressed. For a sentence like the one in (249), for example, she suggests the need of having the context presented above the example in (249) in order to get wide scope. The elements in capital letters bear a high pitch in the pronunciation.

$((\neg A) \wedge(\neg B))$. Other authors, such as Siegel (1987), refers to this as "narrow" scope, instead. 
(249) Context: Everybody knows that chameleons can move their eyes independently, as figure 4 shows; but the situation is different for humans, as figure 5 shows.

The LEFT eye CAN'T go UP and the OTHER one DOWN.

(Winkler 2005: 202)

Moreover, she supports a denial analysis for wide scope looking at prosody. In the wide scope reading also the negation is marked with a high pitch in the pronunciation (250a), while that is not the case for distributed scope, as we can see in (250b).

(250) a. Leon CAN'T eat CAVIAR and Anna BEANS.

$$
\left(\mathrm{H}^{*}\right) \quad \mathrm{H}^{*}+\mathrm{L} \quad \mathrm{H}^{*}+\mathrm{L} \mathrm{H} \quad\left(\mathrm{H}^{*}\right) \mathrm{H}^{*}+\mathrm{L} \mathrm{H} \%
$$

b. The LEFT eye can't go UP and the OTHER one DOWN. $\mathrm{H}+\mathrm{L}^{*} \quad \mathrm{H}^{*}+\mathrm{L} \% \quad \mathrm{H}^{*}+\mathrm{L} \quad \mathrm{H}^{*} \mathrm{LL} \%$ (Winkler 2005: 200, 201)

The same ambiguity is also found with adverbs. In the example in (251) that follows, modified by Potter et al. (2017) from an example by Oehrle (1987), we can see that the frequency adverb "often" can only be interpreted as distributed with the context given, otherwise it could have been ambiguous between the two.

(251) a. Kim has often gone to the beach and Valerie to the city.

b. Context: Kim and Valerie are roommates. Kim goes to the beach very often and Valerie goes to the city all the time. However, these things never happen at the same time, such 
that one or the other is always sitting at home, playing Xbox.

c. Wide scope OFTEN $(\mathrm{P} \wedge \mathrm{Q})$ : False

d. Distributive scope OFTEN $P \wedge$ OFTEN $Q$ : True

(Potter et al. 2017: 1131)

Wide scope of elements such negation and adverbs can also take distributed scope over the conjuncts and this is something that will be important to take into account in the choice of a small or largeconjunct coordination.

We will see next some more properties that relate gapping to information structure. This aspect will be relevant for several analyses we will go through in 4.2.2.

\subsubsection{Gapping and information structure}

As we already saw in the previous section, when talking about the scope of elements over the conjuncts, intonation and its relation with information structure can play an important role.

Intonation, in fact, encodes that the remnants left behind in the second conjunct represent new information. Kuno (1976), as reported in (252), suggests the presence of a "novelty condition on remnants" (see also Hankamer 1979, Sag 1976, Levin and Prince 1986, Hartmann 2001, Kehler 2002, Winkler 2005, Toosarvandani 2013). 
Kuno's Novelty Condition on Remnants

The phrases in the coordinate that are left over from Gapping introduce new information.

(based on Kuno 1976: 310)

Based on this assumption, remnants are considered to each contain focus. Moreover, a second condition on the remnants is related to the presence of contrast between the remnants and the elements present in the antecedent, as reported in (253) by Winker (2005).

\section{(253) Contrastive focus principle}

In gapping the deleted elements must be given. The remnants must occur in a contrastive relation to their correlates.

In the literature, the notion that contrast in gapping relates to the connection between gapping and multiple wh-questions (Sag 1976, Neijt 1979, Pesetsky 1982) is quite consistent. The presence of a multiple wh-question generates a set of alternatives and therefore the possibility of having elements contrasting with each other ${ }^{23}$. This is exemplified in a question like (254A) that can elicit gapping, as we can see in (254B). In (254A), the two wh-elements generate sets of alternatives that are then contrasting in each conjunct. The arguments of the first conjunct are contrasting with the ones of the second one:

\footnotetext{
${ }^{23}$ I will not enter the discussion concerning the differences between contrast and focus. I assume a question under discussion approach that generates sets of alternatives and the parallelism between the conjuncts to create contrast. For more information on this topic, I address the reader to Vallduví \& Vilkuna (1998), Umbach (2004) and Repp (2010).
} 
"John" with "Mary" and "apples" with "banana". In addition, with respect to the two sentences contrasting with each other, Repp (2016: 1) asserts that "S1 and S2 may be construed as being in a contrastive relation if S1 contains an element $\alpha$ that can be construed as an alternative to an element $\beta$ in $\mathrm{S} 2$, where being construed as an alternative reflects the notions of juxtaposition and comparison". (254B), again, is a clear example of this.

(254) (A: Who bought what?)

B: JOHN bought APPLES and MARY BANANA.

(Winkler 2005: 192)

In the literature, then, based on Kuno's (1982: 141) Sorting Key hypothesis, which states that "in a multiple wh-word question, the fronted wh-word represents the key for sorting relevant pieces of information in the answer", Winkler (2005) proposes the contrastive topic and contrastive focus principle. With this principle the author proposes that the external arguments in each conjunct are realized as contrastive topics, while the internal ones are contrastive foci (255). In Winkler's proposal, Kuno's sorting key corresponds to contrastive topic and the second remnant to contrastive focus.

(255) Contrastive Topic and Focus Principle (Winkler 2005: 192) In gapping, the first remnant is a contrastive topic, the second remnant a contrastive focus. The gapped elements must be given. 
Schwabe (2000) follows the same line and assumes the presence of a focus operator for each remnant and corresponding alternatives. For the subjects of each conjunct, though, the focus operator is placed in the functional projection TopP (Topic Phrase). For a more detailed analysis, see Schwabe (2000: 253). Other researchers such as Coppock (2001) and Toosarvandani (2013), instead, assume both remnants to be focalized.

The presence of contrastive focus and contrastive topic on the remnants and the respective alternatives in the antecedent are not detected just from a discourse point of view, but also from a prosodic one. As we already anticipated in the previous section (4.2.1) when looking at how to establish the type of scope of negation present in gapping, examples from Winker (2005) were discussed where prosody was taken into account. The presence of a high pitch in the contrasted elements can be detected. Hartmann (2000) also supports the fact that the contrasting elements are accented, while that is never the case for the verb, which is deaccented, since it is given, and can be deleted in the second conjunct.

On the basis of the properties we described till now, we will next review the main proposals that have been made for gapping.

\subsubsection{Approaches to gapping}

In the analyses proposed for gapping, one of the main questions that are addressed is the size of the conjuncts that are coordinated, either small, referring to $\mathrm{VP}$ or vP coordination, or large, including $\mathrm{TP}$ or $\mathrm{CP}$ coordination. Therefore, in the following two subsections we will 
distinguish two main types based on the size of the conjuncts, and within each type we will see the different solutions that have been provided to account for the material that goes missing and the relation between the remnants and their alternatives in the antecedent. We will see first the proposals that have been made assuming smallconjuncts coordination (4.2.2.1) and then the ones that justify a largeconjunct coordination (4.2.2.2). We will also see that there are approaches that put together both approaches.

\subsubsection{Small-conjunct coordination}

Based on the properties we saw in the previous sections (4.1), it is possible to argue for the presence of coordination of VPs or vPs in gapping. The main arguments used to support this view are the presence of wide scope of modals and cross-conjunct binding. Johnson (2009, a.o.), Winkler (2005), Coppock (2001) and Toosarvandani (2013), among others, support this approach in relation to the size of the conjuncts, but they have different treatments for the material that is missing in the second conjunct. The main proposals related to this approach to coordination in gapping are movement accounts and deletion accounts.

Johnson (2009, a.o.), bases his analysis on justifying: i) the difference between gapping and other types of ellipsis like VPE and stripping in English, and ii) the presence of wide scope of negation and of modals in gapping in English. In order to account for this data, Johnson assumes the presence of $\mathrm{vP}$ coordination, with the negation scoping over both conjuncts. Johnson (2014: 27) generalizes 
claiming that "[i]f a modal or negation Gaps with a following verb, then it may scope over the coordination or be understood in each conjunct". For the sentence in (256), he therefore proposes the structure in (257).

(256) Ward hasn't prepared natto and his guest kumquats.

(Johnson 2004: 64)

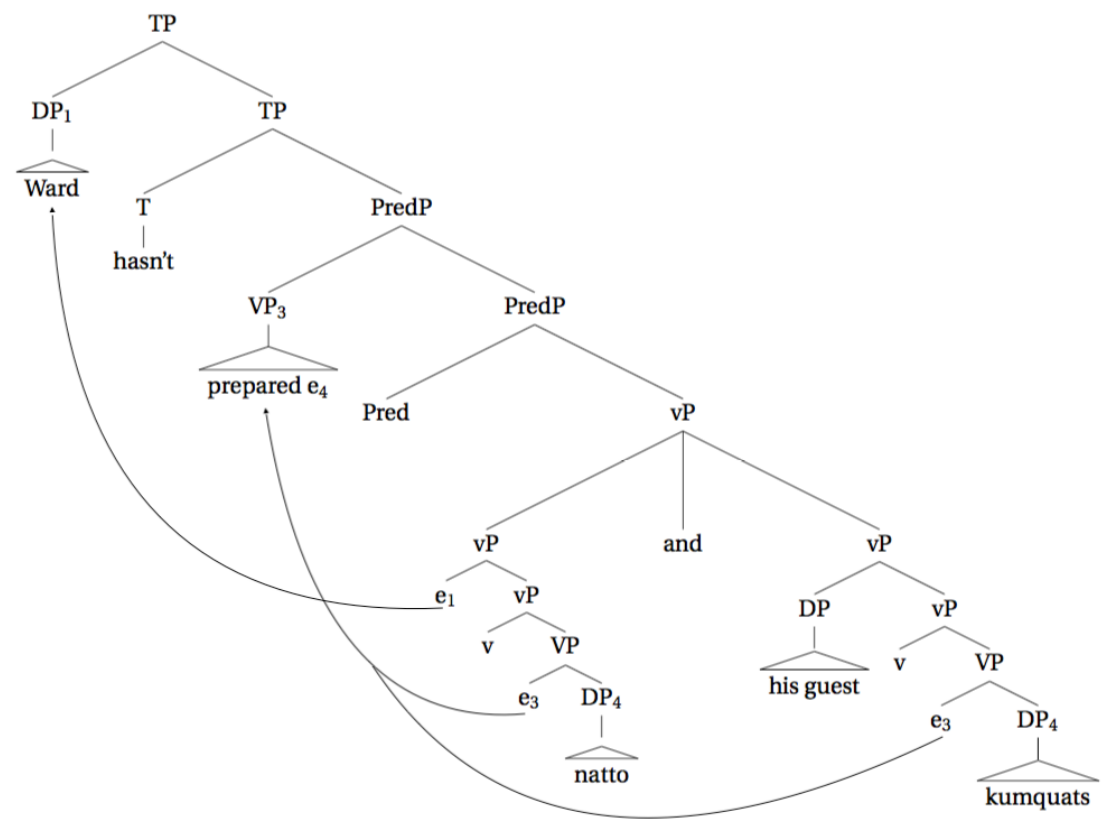

The structure in (257) would also account for cases of cross-conjunct binding, as in the example that we can see in (258), repeated from (245), where we saw the difference with VPE that does not allow this phenomenon. Assuming that binding cannot take place over full clauses, the presence of $\mathrm{vP}$ coordination is then further supported. 
(258) No one's duck was moist enough or his mussels tender enough.

In (257), gapping is conceived as two vPs coordinated under TP and Predicational phrase (PredP) that hosts the verb moving out from both conjuncts via ATB movement. The subject in the first vP reaches the specifier of TP for EPP reasons while the one in the second conjunct stays in-situ. This movement appear to be an instance of violation of Ross's (1967) Coordination Structure Constraint which does not allow movement out of only one conjunct. Lin (2001), though, adopting Ruys's (1992) view that the Coordination Structure Constraint is a semantic constraint, she proposes that the movement of the subject in the first conjunct is semantically vacuous and therefore allowed (see Johnson (2004: 4149) for more discussion about it).

Differently than Johnson, Winkler (2005) derives gapping through sideward movement in which "the computational system copies a given constituent $\alpha$ of a syntactic object $\mathrm{K}$ and merges $\alpha$ with a syntactic object $\mathrm{L}$, which has been independently assembled and is unconnected to K" (Nunes 1995: 182). Moreover, Winkler (2005) accounts for the contrastive relation between the remnants assuming that they move to an A-bar position inside vP (see also López \& Winkler 2002). After the movement of the remnants to TopP and FocP inside $\mathrm{vP}$, sideward movement of the empty VP applies. The coordinate structure, then, builds up with subsequent PF-deletion of the lower vP-copies (Winkler 2005: 181). Winkler uses the scope 
facts to justify the presence of $\mathrm{vP}$ coordination and intonational evidence in relation with the dislocation of wh-phrases and topicalized phrases to support the position of TopP and FocP inside vP.

Coppock (2001), though, goes against the movement accounts, especially Johnson's account, and supports the presence of deletion. Coppock (2001) focuses on the possibility in gapping, as in VPE, to disambiguate scope, following Fox (2000), and anaphora noticing that "the Many-Pronoun Puzzle" by Fiengo \& May (1994) is present also in gapping, and therefore follows from deletion. As for the data on scope, in (259) we can see that in gapping (259b) it is possible to only have surface scope, and not both surface and inverse scope, as in (259a).

(259) a. A student accompanied every visitor $(\forall>\exists),(\exists>\forall)$

b. A student accompanied every visitor yesterday, and Mr. Johnson, today. $*(\forall>\exists),(\exists>\forall)$

Based on Fox's (2000) Ellipsis Scope Generalization in (260), Coppock (2001) concludes that inverse scope in gapping is not possible because it is indistinct from surface scope. Therefore, for Scope Parallelism, ATB movement could not account for these facts.

(260) The Ellipsis Scope Generalization (Fox 2000)

In constructions that involve phonological reduction or deletion, inverse scope is possible only if it is semantically distinct from surface scope both in the sentence that includes 
the phonologically reduced/elided VP and in the sentence that includes the antecedent VP.

Moreover, Coppock (2001) also looked at identity and following Merchant (2001), she adapted to gapping the e-GIVENness condition we saw in 4.1.1.2, by assuming the remnants in the second conjunct and the respective antecedents in the first conjunct to be focus marked. For the sentence in (261), Coppock (2001) presents the focus closure of the gapped clause and of the antecedent clause, as we can see in (262). Being the two identical, they entail one another and therefore deletion is licensed.

(261) $\left[\alpha \mathrm{John}_{\mathrm{F}}\right.$ likes caviar $\left.\mathrm{F}\right]$ and $\left[\gamma\right.$ Mary $_{\mathrm{F}}$ beans $\left.\mathrm{F}\right]$.

(262) a. F-clo $(\alpha)=\exists x \exists y[x$ likes $y]$

b. F-clo $(\gamma)=\exists x \exists y[x$ likes $y]$

(Coppock 2001: 7)

Another important contribution by Coppock (2001) is confirming the presence of structure in the gapped site. She examines different types of "propositional islands", following Merchant (2001), in order to show that gapping is sensitive to them and, therefore, that structure is detected, as we saw in 4.1.1.2 also for VPE and fragment answers. We can see an example for gapping in (263) (for more discussion on this, see Coppock (2001: 12)).

(263) *Suzy doesn't like men who play instruments, and Mary, sports.

(Coppock 2001: 12) 
Another point against Johnson's proposal consists in the fact that he does not take into account the presence of focalized elements in his structure. Toosarvandani (2013: 22), in fact, proposes the Focused Remnant Requirement that states that "the remnants in gapping must each contain a focus". He assumes the need of having the remnants undergoing movement outside the $\mathrm{vP}$ in order to account for information structure, but, supporting the presence of $\mathrm{vP}$ coordination, he excludes the movement of the remnants to SpecTP. The structure he adopts for the sentence in (264) is the one in (265).

(264) Some had ordered mussels, and others swordfish.

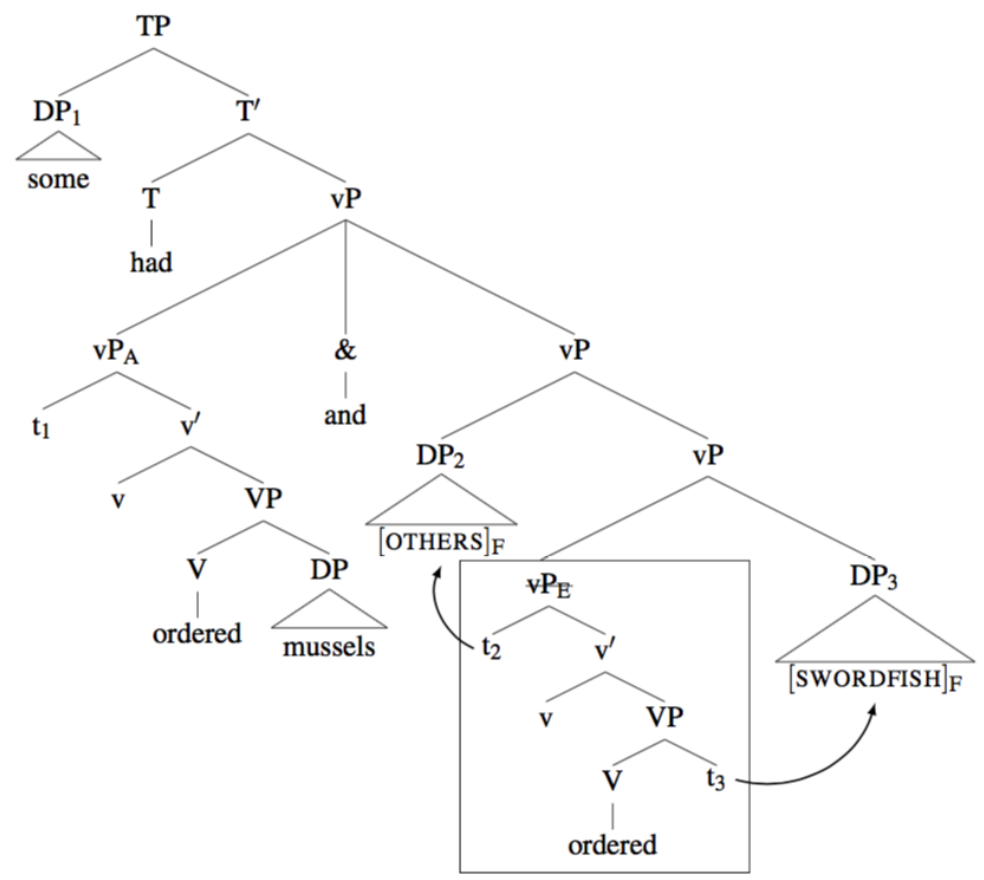

(Toosanvardani 2013: 34) 
Toosarvandani (2013), in reporting the intonation on the elements in the two conjuncts, actually follows several authors, among which Winkler (2005), and agrees on the presence of pitch accent also on the phrases corresponding to the remnants in the first conjunct. The structure in (265), however, does not account for this aspect.

In the next section we will review the approaches that support a large-conjunct coordination and present the main analyses that account for the missing material in gapping.

\subsubsection{Large-conjunct coordination}

Sag $(1976,1980)$ has been one of the first, next to Pesetsky (1982) and Jayaseelan (1990) and others, to propose a unified account for VPE and gapping assuming the presence of large-conjunct coordination and movement of the remnants. Sag (1976), for example, proposed heavy NP shift of the remnant object outside of VP and further deletion of the VP. In (267) we can see the representation of the second conjunct of the sentence in (266), adapted by Johnson (2009), in which the material that will be deleted is marked with a box. 
(266) Some have served mussels to Sue and others swordfish.

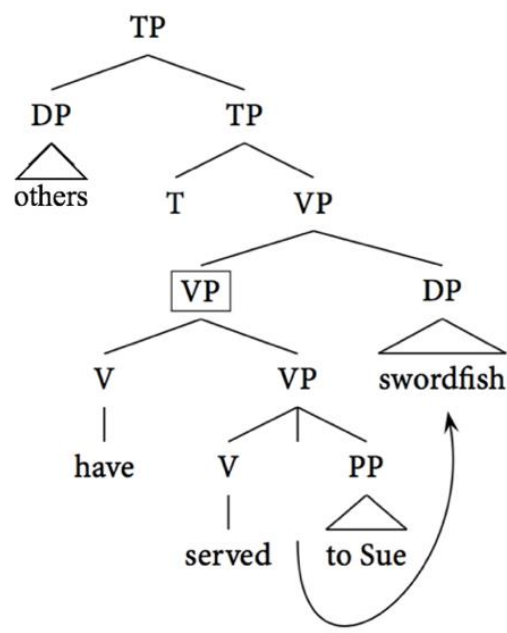

Other authors such as Wilder (1995, 1996) and Hartmann (2000) build their analysis on deletion, but they do not assume the presence of movement. Hartmann (2000), differently than Wilder (1995, 1996), who mainly focus on the identity condition between the gapped site and the antecedent, also takes into consideration the prosodic realization of elements in gapping as an important factor. Hartmann, assuming the presence of deletion at PF, develops two main conditions: i) finite-first condition, which states that "the finite (part of the) verb is obligatorily left out in a non-first conjunct" (Hartmann 2000: 156) and ii) maximal contrast principle in which "the remnants and the correspondents must form a maximal number of contrasting phonological phrases" (Hartmann 2000: 144). Following these two principles, it is not possible to find the verb in the first conjunct accented and in the second one the verb elided. As pointed out by Repp (2009), though, Wilder (1995, 1996) and 
Hartmann (2000) do not give a clear explanation of the facts related to distributed scope of negation, an important argument to deal with when assuming large-conjunct coordination.

Based on the necessity of accounting for the presence of distributed scope of negation and mapping the presence of focalized elements onto the syntactic structure, authors like Gengel (2006) for English and Farudi (2013) for Farsi propose a structure in which the remnants in the second conjunct move to TopP and FocP. Moreover, they suggest the presence of deletion of the whole TP that is left empty in the second conjunct. For the identity condition, they follow Merchant (2001), even though they treat the E-feature slightly differently. A similar analysis has been proposed also for Chinese (Ai 2014), but I will present here only the ones by Gengel (2006) and Farudi (2013), starting from the latter.

Farudi (2013) investigates gapping in Farsi and proposes movement of the remnants in the second conjunct to TopP and FocP, in the sentence left periphery, making then the whole TP available for deletion. She does not assume any movement to the left periphery for the elements in the first conjuncts, though. As for the identity condition, Farudi (2013) adopts Merchant's e-GIVENness approach, but instead of placing the E-feature in the head of the projection whose sister will be deleted at PF, she places it in the head of coordination phrase (\&P), partially following Aelbrecht's (2009) proposal that ellipsis is licensed through agreement. Farudi (2013) suggests that ellipsis is licensed by agreement between the coordinate head ( $\left.\&_{\text {GAPPING }}\right)$ and TP directly, thus satisfying the uninterpretable feature requirement in $\&_{\text {GAPPING }}$ agreeing directly with TP. The E- 
feature "would not delete its complement constituent; rather, $E$ would have to be characterized phonologically as deleting the constituent with which it agrees" (Farudi 2013: 224). The movement of TP to the Spec\&P is justified by the need of satisfying locally the strong uninterpretable TP feature in $\&$ and therefore justify its deletion. For the example in (268), Farudi (2013: 234) proposes the structure at LF in (269).

(268) Git nun xord va Artur berenĵ.

(Farsi)

Giti bread ate.3sG and Arthur rice

'Giti ate bread and Arthur rice.'

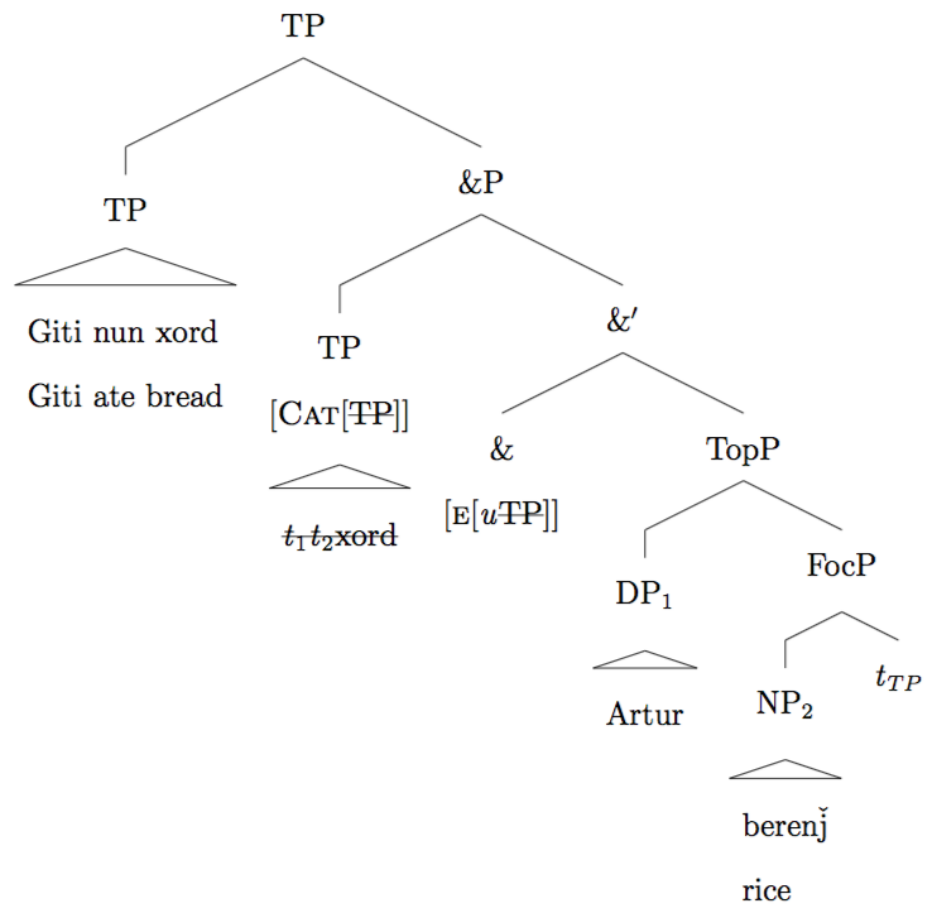


Differently than Farudi, Gengel (2006) remains closer to Merchant's (2001) analysis. Starting from an extensive work on pseudogapping, Gengel applies her analysis also to gapping. Like Farudi, Gengel (2006) also puts forth movement to TopP and FocP only of the remnants in the second conjunct. Compared to other accounts, she also assumes the presence of a [contrastive] feature in the head of TopP and of FocP. The need of checking this feature makes the object and the subject in the second conjunct move up. As we can see in the structure in (271) for the sentence in (270), not just the subject, but also the object, needs to move up to SpecvP (Lasnik 1995, 1999 uses SpecAgrO), where it checks EPP features before moving up to FocP.

(270) Claire read a book and Heather a magazine.

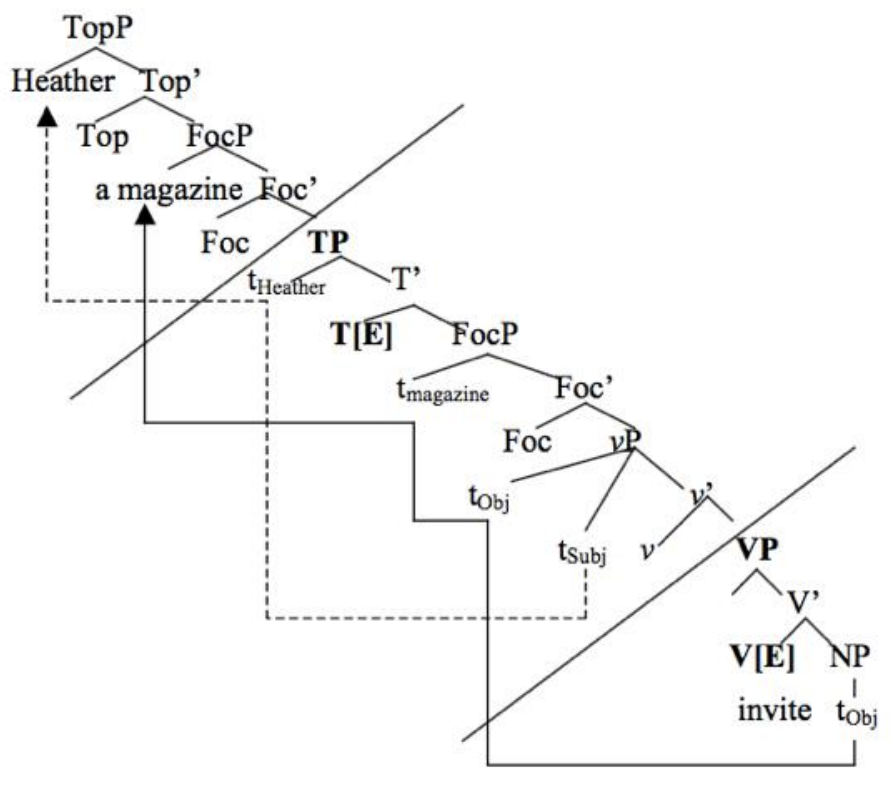

(Gengel 2006: 15) 
As for the identity condition, Gengel (2006) assumes, too, that eGIVENness is involved considering the presence of E-feature but, differently than Merchant (2001) and Aelbrecht (2009), she places it in the head of the projection that will be targeted for deletion, for gapping the whole TP.

Finally, other accounts have been proposed in which the presence of both small and large-conjunct coordination is assumed. Jung (2016), looking at gapping in Korean and Spanish, suggests that there are two types of gapping: one involves large-conjunct coordination and deletion at PF, while the other needs small-conjunct coordination and it can be either the result of PF-deletion or the product of ATB-movement. Jung (2016) bases his analysis mainly on agreement, intonational contour and scope of negation. Potter et al. (2017) consider the need as well of having both small and largeconjunct coordination and they take into consideration the ambiguous scope of adverbs between wide and distributed scope, where the first type needs a large-conjunct coordination, while the distributed scope requires a small-conjunct one.

We will see next the data and the accounts that have been reported for gapping in SLs, even though not much research has been done on this topic.

\subsubsection{Gapping in SLs}

There is not much work done on ellipsis in SLs, but from the data presented for LIS (Cecchetto et al. 2015), ASL and LSF (Schlenker 2014) for VPE and for gapping for ASL (Liddell 1980, Frazier \& 
Yosida 2012), FinSL (Jantunen 2013) and HKSL (Tang \& Lau 2012), it is possible to see that it is a consistent phenomenon also in SLs.

Focusing on gapping ${ }^{24}$, Liddell (1980) for ASL, Tang \& Lau (2012) for HKSL and Jantunen (2013) for FinSL provide some descriptive data on how gapping is expressed. In (272) we can see that also in these languages the gap is present in the second conjunct, irrespective of whether the language is head initial like ASL or head final like HKSL and FinSL. Therefore, the definition of gapping by Johnson (2014) that we presented in 4.2.1 holds also for SLs.

(272) a. HAVE WONDERFUL PICNIC. PRO.1 BRING SALAD,

$\frac{\mathrm{hn}}{\mathrm{JOHN}}$ SANDY $\frac{\mathrm{hn}}{\mathrm{CHICKEN}}$ TED $\frac{\mathrm{hn}}{\mathrm{HAMBURGER}}$

'We had a wonderful picnic. I brought the salad, John

(brought) the beer, Sandy (brought) the chicken and Ted

(brought) the hamburger.'

(Liddell 1980: 31)

b. TOMORROW PICNIC, IX 1 BRING CHICKEN WING,

(HKSL)

PIPPEN SANDWICHES, KENNY COLA,
$\frac{\text { bl forward }+\mathrm{hn}}{\text { bl forward }+\mathrm{hn}}$

CONNIE CHOCOLATE.

'(We) will have a picnic tomorrow. I will bring chicken

wings, Pippen (brings) sandwiches, Kenny (brings) cola,

(and) Connie (brings) chocolate.' (Tang \& Lau 2012: 347)

\footnotetext{
${ }^{24}$ In this section I will present only the data on gapping described in the literature for SLs. I will present the ones on VPE on LIS when comparing gapping and VPE in LSC in 5.1.2. Moreover, in 5.5, where I will give an account for LSC about the type of identity present in gapping, I will also introduce the tests used in LIS, ASL and LSF (Cecchetto et al. 2015; Schlenker 2014).
} 
c. GIRL HAS.GOT TWO PIECES BOY ONE-PIECE.

'The girl has two and the boy (has) one.'

(Jantunen 2013: 317)

Differently than LSC, as we will see in 5.1, gapping in HKSL is not allowed with agreeing and CL verbs, as in (273).

a. ${ }^{*} \mathrm{KENNY}_{0} \mathrm{SCOLD}_{3}$ BRENDA, PIPPEN Ø CONNIE

(HKSL)

'Kenny scolds Brenda (and) Pippen Ø Connie.'

b. * IX 1 HEAD WALL $\emptyset$, BRENDA HEAD WINDOW

CL:HEAD-BANG-AGAINST-FLAT-SURFACE

'I banged my head against the wall and Brenda against the window.'

(Tang \& Lau 2012: 348)

Moreover, as we can see in the examples in (272), the NMMs that characterize this structure are different in ASL and HSKL, but they spread on the same elements. Liddell (1980) noticed that in ASL a head nod is produced on the remnant object, while in HKSL there is an additional forward body lean. Looking at the NMMs showing contrast between the elements in the conjuncts in gapping in ASL, Frazier \& Yosida (2012) found different data than Liddell (1980). In (274), we can see the brackets marking the spreading of the NMMs, even though Frazier \& Yosida do not specify which ones are used. In the examples in (274) it is clear that the NMMs need to spread over the two conjuncts including both subject and object. This evidence is used by Frazier \& Yosida (2012) to support a large-conjunct coordination approach for gapping in ASL. 
(274) a. [IX1 LIKE COFFEE], [IX2 TEA].

b. *IX1 [LIKE COFFEE], [IX2 TEA].

c. *IX1 LIKE [COFFEE], [IX2 TEA].

'He likes coffee, and she, tea.'

(Frazier \& Yosida 2012: 13)

So far, Frazier \& Yosida (2012) have been the only researchers who attempted a syntactic analysis of gapping. In the next chapter we will see more data on NMMs in gapping in LSC, and we will arrive at the same conclusion to which Frazier \& Yosida (2012) got to for ASL, but will provide a more detailed analysis that also involves information structure.

\subsection{Summary and conclusions}

In this chapter we went through the main analysis proposed in the literature for ellipsis and gapping, in particular. We first answered the two main questions that have been asked about the different types of ellipsis identified, such as sluicing, gapping, stripping, fragment answers, VP ellipsis, pseudogapping and NP ellipsis. To the question "is there unpronounced syntactic structure in the ellipsis site?", two main approaches have been taken: the non-structural and the structural one. The first one assumes the absence of structure in the ellipsis site while the second one, thanks to tests like island sensitivity, defends its presence. Related to the first question, another important issue is whether the identity between the ellipsis site and 
its antecedent is syntactic or semantic. To answer this last one, we saw that there are arguments that support both options.

We focused then on gapping. We first presented the properties that make this elliptical structure in English and in most languages "unique" due to the fact that it can only appear in coordination and that, unlike VP-ellipsis, with respect the no embedding constraint. For the last point, Farsi is an exception (Farudi 2013). As we will see in the next chapter, gapping in LSC behaves like Farsi and it can also appear in subordination. Other properties such as the scope of negation and modals can influence the analysis of gapping, together with the contribution of information structure. One of the most important issues in the analysis of gapping, in fact, is determining the presence of large (CP/TP) or small conjuncts (vP/VP) coordination. Starting from this distinction, different analyses have been proposed, among which ATB movement of the verb (Johnson 2004, a.o.), or deletion (Coppock 2001, Gengel 2006, Farudi 2013) are the more discussed. Scope of negation and information structure will be crucial in determining a large conjuncts coordination structure for LSC.

Finally, we went through the data on gapping in ASL, HKSL and FinSL, and presented the attempted analysis for gapping in ASL by Frazier \& Yosida (2012), too. The same conclusion reached for ASL will be supported also for LSC, even if different means will be used.

After presenting this data, we will next look thoroughly into gapping in LSC describing its properties and proposing a syntactic analysis that will fit the one proposed for coordination seen in chapter 3 , where the structure is right-branching. 


\section{GAPPING IN CATALAN SIGN LANGUAGE}

In this chapter we will present the properties of gapping in Catalan Sign Language (LSC), providing a syntactic analysis for it. In 5.1 and 5.2 we will put in contrast the properties of gapping with the ones of other types of ellipsis, in particular VP ellipsis (VPE). That will help to give a unified account for both structures in LSC. In order to provide an analysis for gapping, in 5.3 we will also describe the discourse properties of gapping in LSC, in line with the analysis presented for spoken languages in 4.2.1.1. We will give a detailed description of the NMMs found for contrastive topic and contrastive focus. On the basis of this data, the analysis that will be proposed in 5.4 justifies the presence of large-conjunct coordination and deletion at PF of the whole TP in gapping and deletion of $\mathrm{vP}$ in VPE, in line with Merchant (2001, 2013), Coppock (2001) and Gengel (2006). After giving the syntactic analysis, we will enter the description of the facts related to the identity condition that allows deletion and will show that there are cases in which not only syntactic but also semantic identity is required. In the last section in 5.6, we will focus on the directionality of gapping and its relation with the directionality of the coordination structure showing that the former depends on the latter.

\subsection{Ellipsis in LSC}

In LSC, ellipsis is attested, as in spoken and other sign languages. It is possible to find different types of ellipsis, depending on the part of 
the structure that gets affected by it. As presented in 4.1 for spoken languages, in LSC it is also possible to identify VP-ellipsis, stripping, pseudogapping and gapping, among other types of ellipsis. Let us see some examples in (275).

(275) a. MARINA FRUIT 3-GIVE-1 JORDI 3-AUX-1

VPE

FRUTT 3-GIVE-1 ALSO (extra_VPE)

'Marina gave me some fruits, and Jordi did, too.'

b. MARINA CHOCOLATE EAT CAN JORDI

stripping EHOCOLATE EAT CAN ALSO. (68(+67).20)

'Marina can eat chocolate, and Jordi, too.'

c. FRIEND IX-1-POSS FLOWER 3-GIVE-1 pseudogapping JORDI BRACELET 3-AUX-1 GIVE. (66.1.02)

'My friend gave me flowers and Jordi did (give me) a bracelet.'

d. FRIEND IX-3-ARC FLOWER 3-CL:GIVE-1 gapping JORDI WATCH 3-CL:GIVE-1. (56.14) 'Some friends gave me flowers and Jordi a watch.'

In the example in (275a) for VPE, we can see the use of an agreement verb in the first conjunct and the use of an auxiliary, glossed as AUX, as remnant in the second conjunct, together with the subject. The function of AUX in LSC is used to mark [+person] feature agreement of arguments (Quer 2011), as in other SLs it is said to mark agreement with subject/object (Pfau \& Steinbach 2008). In LSC it is used with plain verbs to mark agreement when the arguments bear the feature [+human] or as in the case in (275a), if the verb is deleted but the 
relation between the arguments needs to be marked. Assuming the auxiliary to be in the head of TP, the part of the structure affected in (275a) is the whole vP, following Merchant's (2013b) account. Moreover, the focus additive marker ALSO, corresponding to also or too in English is used. As we saw in 3.1.1.2, ALSO can function as coordinator as well, but when placed in final position in the sentence, it is used as focus additive marker. As we will see in 5.3, the presence of ALSO supports the discourse properties of both VPE and gapping. In (275b), instead, we have an example of stripping in the second conjunct of which everything is deleted apart from one constituent, following Hankamer and Sag (1976). (275c) is a case of pseudogapping, as called by Stump (1977) for English and, after him, Levin (1986). Finally, (275d) is an example of gapping, whose properties in LSC we will see in detail in 5.2.

The classification in (275) for LSC is based on the assumption that the head of $\mathrm{T}$ is always active, either with agreement or with plain verbs. For LIS, though, Cecchetto et al. (2015: 217) do not follow the same assumption. They assume that, in LIS, the verb never moves from V. From this I conclude that for LIS, examples that on the surface could look like stripping (with the whole TP involved), have to be considered cases of VPE, instead, like the one in (276).

(276) Gianni Maria LiKe. Piero SAME.

'Gianni likes Marina. Piero does, too.'

(Cecchetto et al. 2015: 221) 
In the next section we will discuss in more detail how agreement can affect the classification of ellipsis in LSC.

\subsubsection{Agreement in LSC and classification of ellipsis}

In SL, following Padden (1983/1988), three main classes of verbs have been identified: a) plain verbs, which do not agree in space with the arguments, b) agreement verbs, which agree with subject and object, and b') spatial verbs, which agree in space with locative referents. Janis (1992), for ASL, and Quadros (1999), for Libras, use a different model, though, where they do not consider differences among agreement and spatial verbs. Quadros \& Quer (2008), as well, suggest treating spatial and person agreement in the same fashion due to the fact that a referential locus can carry both [+person] and [+location] features. Moreover, Quadros \& Quer (2008) state that "agreement is understood as the movement between two points associated with the arguments of certain verbs". Therefore, both classes use a morpheme that realizes agreement between subjectobject loci or locations, PATH, sometimes in combination with facing of the hand as well. For LSC, then, it is possible to classify verbs as plain and agreement verbs. The first class does not show PATH movement to agree with the arguments and if the argument is [+human], agreement is expressed using AUX (e.g., LIKE, LOVE, FORGET). Even though not largely attested, plain verbs in LSC can show agreement through the use of NMMs. As we can see in the example in (277) below, the verb LOVE can be followed by AUX to express the relation between the two arguments or through the use of 
body lean and head shift towards the loci in the space where the referent is localized (277b). Eye gaze seems to play a role as well, as attested for other SLs like ASL, in which head tilt and eye gaze are considered to realize agreement with plain verbs (Bahan 1996, Bahan et al. 2000).

a. MARINA JORDIj LOVE $_{i}$ JUXX $_{j}$. (63.11)

'Marina loves Jordi.'

bl contr. space cont. bs+hs contr.

b. MARINA JORDI LOVE. (63.10)

'Marina loves Jordi.'

Among agreement verbs, which all express agreement through the use of the morpheme PATH in space, it is possible to find two main groups: directional verbs and locative verbs (Barberà \& Mosella 2014). In the first group the arguments that the verb agrees with always bare a [+animate] feature and PATH movement is realized from the subject location to the object location (e.g., EXPLAIN, TEACH $)^{25}$. As for locative verbs, the arguments of the verb include one or more locative referents (e.g., GO). Following Costello's (2016) classification for LSE, it is possible to detect also in LSC a class for one argument agreement verbs, like the case of PAY in LSC, in which the agreement takes place only with the direct object.

\footnotetext{
${ }^{25}$ It is also possible to identify backward and reciprocal verbs in the class of directional verbs. In backward verbs the movement is from the object to the subject locations, like in UNDERSTAND. Reciprocal verbs, instead, show agreement with both arguments that are affected by the action (e.g., EMAIL-EACH-OTHER).
} 
Recent accounts of agreement in SL follow a minimalist approach (Pfau et al. 2018; Costello 2016) and they assume that in SLs the verb moves to $T$ via $\varphi$-feature checking, in a different way depending on the verb class, but there is always material that reaches T. Pfau et al. (2018) analyze agreement as the movement of complex heads to T. Their formation depends on the type of verb (e.g., plain verbs vs. agreement verbs) that affects the head formation and therefore the type of complex head moving to T. Costello (2016), instead, considers head movement of $\mathrm{V}$ to $\mathrm{T}$, that is present with all classes of verbs. In the case of plain verbs, agreement is manifested morpho-syntactically, but not phonologically. One of the main explanations used by Pfau et al. (2018) for justifying the movement of the verb is the fact that the expression of negation in DGS can be realized with a headshake spreading over the verb, showing the need to have the verb moving to NegP above TP. For LSC, the case is the same (Pfau \& Quer 2007). No other standard tests can be applied to SLs that are head-final. For example, looking at the position of adverbs, as it is usually done for spoken languages, is not an option, since in head-final SLs the adverb is always in sentence final position. Both Pfau et al. (2018) and Costello (2016) follow Chomsky (2000, 2001), where agreement is considered a syntactic operation triggered by the checking of certain features that cannot be interpreted at the interfaces. They are therefore unvalued and uninterpretable. These unvalued phi-probes are on $\mathrm{v}$ and $\mathrm{T}$ and must be valued through Agree. The features on $v$ relate to object agreement, and those on $\mathrm{T}$ to subject agreement and they search for a value in their c-command domain. Afterwards, the value of the 
subject is copied onto $\mathrm{T}$ and that of the object onto v. In SLs, agreement verbs always show movement from $\mathrm{V}$ to $\mathrm{T}$ (in DGS for Pfau et al. (2018) and LSE for Costello (2016)). For DGS a movement to $\mathrm{T}$ of complex heads made up of $\mathrm{v}$ and $\mathrm{V}$ to $\mathrm{T}(\mathrm{V} \rightarrow \mathrm{V}+\mathrm{V}$ $\rightarrow[\mathrm{V}+\mathrm{v}]+\mathrm{T})$ is proposed, while for LSE the verb is argued to move from $\mathrm{V}$ to $\mathrm{v}$ till $\mathrm{T}$ checking [number] and [identity] features, which replace both [+person] and [+location] features. We can see an example of the proposal made for DGS in (278) and (278'), and for LSE in (279) with agreement verbs.

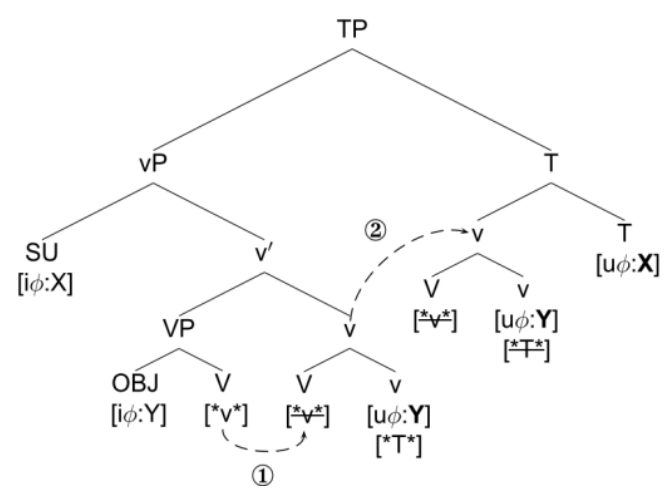

$\left(278^{\prime}\right)$

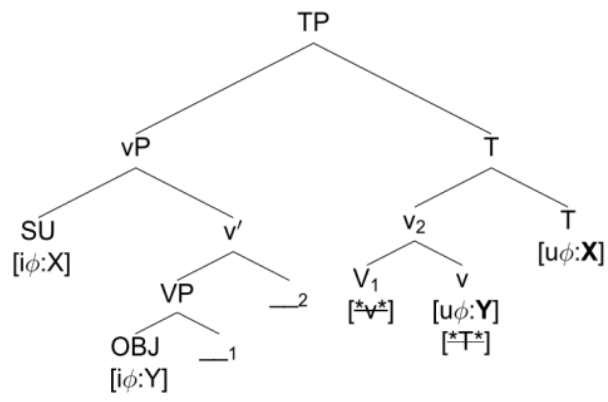


(279) IX $_{\mathrm{x}} \mathrm{xTRICK}_{1}$

'He's tricking me.'

(Costello 2016: 291)

(279’)

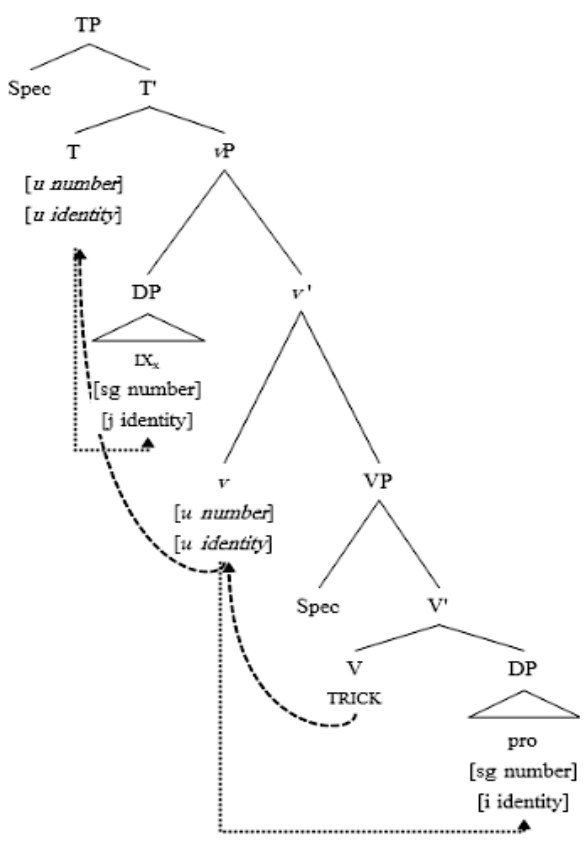

With plain verbs, instead, Pfau et al. (2018) claim that the verb stays put in $\mathrm{V}$, but that $\mathrm{v}$ always moves to T. For DGS, in fact, it is stipulated that an auxiliary, PAM, is always used to express agreement with plain verbs. Therefore, $\mathrm{v}$ is realized in T as PAM, as we can see in (280) and (280') where V stays put, and v moves to T. 
(280)
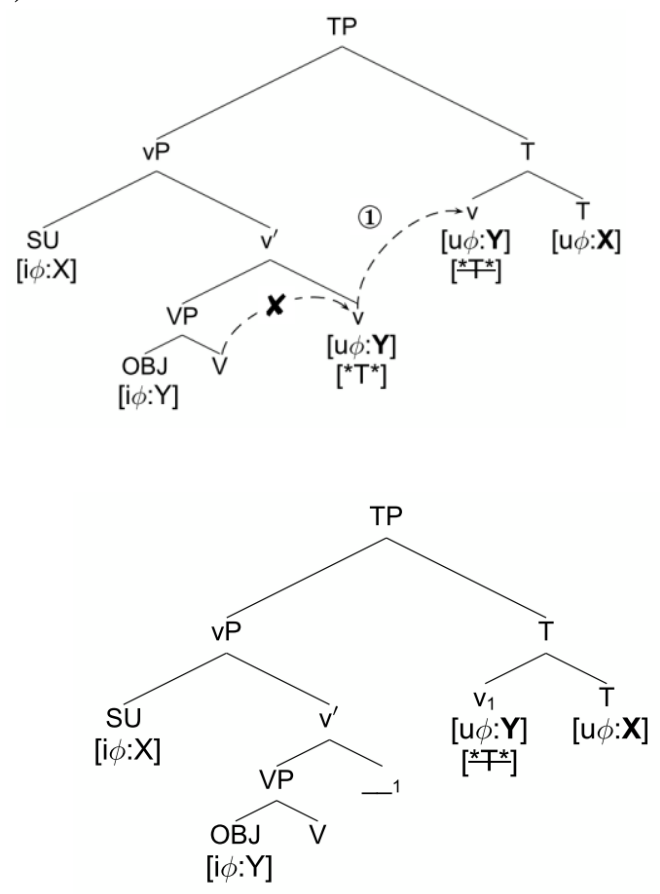

$\left(280^{\prime}\right)$

Costello (2016) does not give a clear account for plain verbs, even though he specifies that there is syntactic agreement even if it is not expressed morpho-phonologically. He specifies that "the fact that non-manual markers may appear in the absence of manual markers of agreement supports the idea that agreement may be present but blocked in (the manual component of) the phonological form" (Costello 2016: 293). Pfau et al. (2018), as well, point out that in languages that do not show an auxiliary to express agreement with plain verbs, non-manual markers can be an alternative strategy to express agreement. In their approach, with plain verbs, $v$ would be expressed in T as NMMs.

In the description of the verb classification, LSC does not fully behave like DGS, that is the auxiliary AUX is not as productive 
as PAM with plain verbs. As we saw in (277b), though, NMMs can be used to compensate for this absence. It is possible to defend, also for LSC, then, that there is always material moving to $\mathrm{T}$, following either Costello (2016) or Pfau et al. (2018) for the syntactic derivation.

For LIS, though, Cecchetto et al. (2015), claim that in LIS there is no verb movement and they use a test based on how word order gets affected in combination with a locative element. In LIS, the verb is assumed to precede functional signs that express aspect, modality, polarity and future tense. Moreover, Cecchetto et al. (2015) notice that when a locative expression like GARDEN IX-LOC is used, it normally appears in sentence initial position (281a). When having an argument of the verb topicalized and a locative expression used in the same sentence, the verb occurs to the left of the locative phrase which is right adjoined to the VP (281b).

(281) a. GARDEN IX-LOC GIANNI VASE BREAK

b. VASE GIANNI BREAK GARDEN IX-LOC
'Gianni broke a vase in the garden.'

(Cecchetto et al. 2015: 216)

In LSC, though, despite the fact that locative expressions are usually found in sentence initial position, it is not possible to reach the same conclusion as for LIS. If an element in the sentence is topicalized, it is not possible to realize the same word order found in LIS, as we can see in (282). 
a. KITCHEN JORDI VASE BREAK. (60.14)

b. *VASE JORDI BREAK KITCHEN IX-LOC. 'Jordi broke a vase in the kitchen.'

Moreover, in LSC it is possible to apply the test used by Pfau et al. (2018) to support the presence of $\mathrm{V}$ to $\mathrm{T}$ movement of the verb by looking at negation. In DGS, negation can be expressed using just headshake as NMM, like in (283), and the same is true for LSC, in (284). In (283) we can see that, in DGS, the NMM for negation spreads either on the verb or on the auxiliary PAM. In LSC, in (284b), they spread on the verb, as well. The headshake is analyzed by Pfau \& Quer (2007) as a non-manual affix placed in the head of NegP, which is hierarchically located above TP, as we can see in the representation in (285) for LSC. The presence of a negative feature [+neg] in the head of NegP imposes an alteration of the prosody that adds headshake on the element bearing this feature, usually the negative marker NOT (NO), as we can see in (284a). If the negative marker NOT (NO) is not present, Pfau \& Quer (2007: 9-10) state that "V-to-Neg raising is triggered by the Stray Affix Filter (Baker 1988), which bans free bound morphemes in syntax, thus triggering movement of another element to support it. Following verb movement, the featural affix attaches to the verb and consequently, headshake on the verb sign only is grammatical in LSC". We can see this exemplified in (284b). Even when the negative sign like NO-RES (nothing) is used, since it is placed in SpecNegP and the verb that needs to move to the head of NegP because NO is absent, the verb shows NMMs spread on top of it, as in (284c). 
Coordination and gapping in Catalan Sign Language (LSC)

(283) a. YESTERDAY POSS 1 MOTHER INDEX 3 a $\frac{\mathrm{hs}}{3 \mathrm{aVISIT}_{1}}$.

(DGS)

'Yesterday my mother didn't visit me.'

b. INDEX 1 NEW TEACHER INDEX 3 a LIKE $\frac{\text { hs }}{1 \text { PAM }_{3 a} \text {. }}$

'I don't like the new teacher.'

(Pfau et al. 2018: 28)

(284) a. SANTI [NegP [vP MEAT EAT $]\left[\frac{\mathrm{hs}}{\left.\mathrm{Neg}_{\mathrm{NOT}}\right]}\left[\operatorname{specNegP}_{\mathrm{Np}} \mathrm{Op}\right]\right]$

b. SANTI [ ${ }_{\mathrm{NegP}}\left[{ }_{\mathrm{VP}} \mathrm{MEAT} \mathrm{t}_{\mathrm{V}}\right]\left[_{\mathrm{Neg}} \frac{\mathrm{hs}}{\mathrm{EAT}]}\left[\mathrm{SpecNegP}_{\mathrm{P}} \mathrm{Op}\right]\right]$

c. SANTI [ $\left.{ }_{\mathrm{NegP}}\left[\mathrm{VP}_{\mathrm{P}} \mathrm{MEAT} \mathrm{t}_{\mathrm{V}}\right]\left[_{\mathrm{Neg}} \frac{\mathrm{hs}}{\mathrm{EAT}}\right]\left[\mathrm{SpecNegP}_{\mathrm{NO}-\mathrm{RES}}\right]\right]$

'Santi doesn't eat meat.'

(adapted from Pfau \& Quer 2007: 10)

(285)

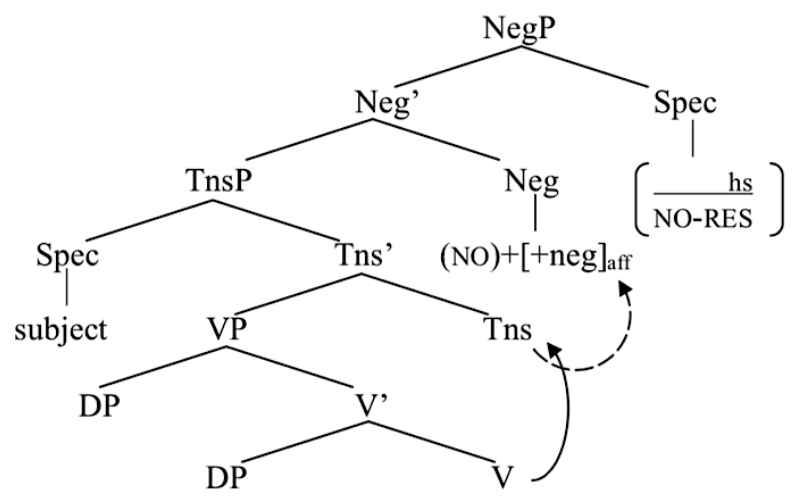

(Quer 2002)

Therefore, also for LSC we can confirm the movement of the verb to T, as applied by Pfau et al. (2018) for DGS. 
The assumption that in LIS the verb stays in V, though, leads to the conclusion that examples like (286a) and more straightforwardly $(286 \mathrm{~b}, \mathrm{c})$ can only be cases of VPE. Instead, if there is material in $\mathrm{T}$ in the first conjunct, which will be deleted also in the second conjunct, we have stripping, as in (287). With stripping, in fact, it is the whole TP that is targeted.

(286) a. GIANNI MARIA LIKE. PIERO SAME.

'Gianni likes Maria. Piero does, too.'

b. GIANNI BOOK BUY MUST. MARIA MUST SAME.

'Gianni must buy a book and also Maria must (buy a book).'

c. GIANNI BEAN EAT FUT. PIERO FUT SAME.

'Gianni will eat beans and Piero will, too.'

(287) a. GIANNI BOOK BUY MUST. MARINA SAME.

'Gianni must buy a book and Maria, too.'

b. GIANNI BEAN EAT FUT. PIERO SAME.

'Gianni will eat beans and Piero, too.'

(Cecchetto et al. 2015: 221-222)

(286a) is the example that puts in contrast LSC and LIS with respect to the movement of V to T. Assuming either Costello (2016) or Pfau et al. (2018), with both plain and agreeing verbs the head of TP is occupied and therefore cases like the ones in (288) in LSC should be considered cases of stripping, while the ones in (289) must be cases of VPE, where only VP is affected. In (288a) there is a plain verb, in 
(288b) a plain one that needs an auxiliary or special NMMs to express agreement, and in (288c) an agreement verb.

'Marina likes stones and Jordina likes stones, too.'

b. MARINA JORDI $_{j}$ LOVE $\left(33_{i}\right.$-AUX- 3 j $)$ JOAN ALSO. (84.05)

'Marina loves Jordi and Marina loves Joan, too.'26

c. MARINA PIZZA PREPARE CAN JORDI ALSO. (66.1.08)

'Marina can prepare pizza and Jordi, too.'

(289) a. MARINA FRUIT 3-GIVE-1 JORDI 3-AUX-1

ALSO. (extra_VPE)

'Marina gave me some fruits and Jordi did, too.'

b. MARINAi JORDI $3_{\mathrm{j}} 3_{\mathrm{i}}$-AUX-3 $3_{\mathrm{j}}$ LOVE IX-1 1-AUX- $3_{\mathrm{j}}$ ALSO. (60.10)

'Marina loves Jordi and I do, too.'

c. $?{ }^{27}$ MARINA FRUIT EAT CAN JORDI CAN ALSO. (66.1.04)

'Marina can eat fruits and Jordi can, too.'

The examples in (288) match, in fact, the definition of stripping given by Hankamer and Sag (1976: 409): "Stripping is a rule that deletes everything under identity with corresponding parts of a preceding clause except for one constituent (and sometimes a clause initial adverb or negative)".

\footnotetext{
${ }^{26}$ When the auxiliary is not produced in the first conjunct, the sentence is more likely to be ambiguous between JOAN being the subject or the object of the second conjunct.

${ }^{27}$ Repeating the modal is perceived as heavy even though it is not ungrammatical.
} 
For LIS, Cecchetto et al. (2015) apply some tests in order to prove that examples like the one in (287a) are cases of stripping. First of all, Cecchetto et al. (2015) make sure that LIS has aux-stranding ellipsis and not verb-stranding ellipsis, using the presence of an auxiliary-like element in the second conjunct in the elliptical clause to distinguish stripping from VPE. Languages like Hebrew, for example, despite having $\mathrm{V}$ to $\mathrm{T}$ movement of the verb, show verbstranding VPE, like in (290) from Doron (1999). Languages like English, instead, are considered to have auxiliary-stranding VPE (291).

(290) a. Q: at saragtet ha-sveder ha-ze

(Hebrew)

Q: you knit ACC-the sweater this

'Did you knit this sweater?'

b. A: lo, ima Seli sarga

A: no, mother my knit

'No, my mother knit it. or No, my mother did.'

(Doron 1999: 128)

(291) Q: Did you knit this sweater?

A: No, my mother did.

LIS and LSC are both languages that show auxiliary-stranding VPE, as we can see in (292a) and (292b, b'), respectively.

(292) a. GIANNI BEAN EAT FUT. PIERO FUT SAME.

'Gianni will eat beans and Piero will, too.'

(Cecchetto et al. 2015: 221) 
b. A: MARINA LOVE 3 -AUX-2?

'Does Marina love you?'

B: NO, JORDI $3 \mathrm{k}_{\mathrm{k}}$-AUX-1.

'No, Jordi does.'

b'. MARINA FRUIT 3-GIVE-1 JORDI 3-AUX-1 ALSO. (extra_VPE)

'Marina gave me some fruits and Jordi did, too.'

Cecchetto et al. (2015) also look at other properties of stripping and VPE that are found in English. In English, VPE can occur in subordination (293a, a') but stripping cannot (293b, b'). In addition, VPE allows for backward anaphora (294a), while stripping does not (Lobeck 1995), as in (294b). The same is found also for LIS (295), where the ellipsis site appears in a subordinate clause and precedes its antecedent, therefore realizing VPE.

(293) a. John left and Mary did, too.

a'. John left, because Mary did.

b. John left and Mary, too.

b'. *John left because Mary, too.

(294) a. John didn't, but Mary bought books.

b. *John too and Mary bought books.

(295) $\frac{\text { if }}{\text { IF PIERO NOT, GIANNI GO. }}$

'If Piero does not, Gianni will go'

(Cecchetto et al. 2015: 224) 
Applying the same test to LSC, it seems that the examples in (288) are actually cases of VPE and not of stripping. As we can see in (296), the ellipsis site can appear in subordination and can realize backward anaphora.

(296) a. MARINA MATH STUDY BECAUSE SISTER IX-3POSS ALSO. (66.1.01)

'Marina studies math because her sister does, too.'

b. IF MARINA NO JORDI ICE-CREAM BUY. (62.13) 'If Marina doesn't, Jordi will buy ice-cream.'

Looking at the properties of stripping and VPE in LSC and English, it seems that Cecchetto et al.'s analysis, in which ellipsis cases like the ones in (286a) are considered VPE, follows also for LSC for the example in (288a). As we will see for gapping in LSC, though, the properties used as a baseline to identify a certain structure in English might not be the same cross-linguistically, as we also saw for the No embedding constraint in Farsi (see 4.2.1).

Moreover, Konietzko (2016) points out that an English sentence like the one in (297) receives an interpretation where John is either the subject or the object of the second clause. Such ambiguity is never found with VPE. In (298), for example, the remnant in the second conjunct can only be the subject.

(297) Mary invited Anna and JOHN, too. (Konietzko 2016: 16)

(298) Mary invited Anna and John did, too. 
As we quickly anticipated in (288b), the same also happens in LSC when the arguments of the verb are [+human]. We can see two more examples in (299), where only the NMMs such as body lean and use of the space can help disambiguate the reading where the remnant in the second conjunct is the subject or the object of the deleted verb. The distinction between the two readings is not always neat, though.
(299)
a. MARINA JORDI HELP JORDINA ALSO. (63.05)
'Marina helps Jordi and Jordina helps Jordi, too.' hl ipsil. ips. space hl+bl contr.
b. MARINA JORDI HELP JORDINA ALSO. (63.06)
'Marina helps Jordi and Marina helps Jordina, too.'

There are two main factors, then, that support the claim that sentences like MARINA STONE LIKE JORDINA ALSO are, in LSC, cases of stripping and not of VPE: i) the attested V to T movement of agreement verbs and anyway the presence of material (AUX or NMMs for agreement) in $\mathrm{T}$, and ii) the ambiguity in interpreting the remnant in the second conjunct as the subject or the object of the deleted verb. The data we showed in this section underline the consequences of assuming a minimalist account for agreement for LSC following Pfau et al. (2018) and Costello (2016) in distinguishing the presence of stripping and VPE. VPE, then, is only present in LSC when an auxiliary element or a modal is left as remnant in the second conjunct. This type of analysis for agreement does not affect the identification of other types of structures such as pseudogapping (275c) or gapping 
(275d), even though it will influence what is going to be considered as deleted material in the second conjunct in gapping, whether vP/VP or TP.

Let us see next the properties of gapping in LSC, the structure that we will focus on in rest of the chapter.

\subsection{Gapping}

In LSC gapping is also attested, as anticipated in 5.1. In (300), repeated from (275), we can see that the verb is removed from the second conjunct (300a) and it can be so also together with other elements (300b), but keeping the contrast between the remnants and the correlate antecedents ${ }^{28}$.

(300) a. FRIEND IX-3-ARC FLOWER 3-CL:GIVE-1 JORDI WATCH

3-CL:GIVE-1. (56.14) 骂

'Some friends gave me flowers and Jordi a watch.'

b. JORDI PASTA EAT FAST JORDI PASTA EAT SLOWLY.

'Jordi eats pasta fast and Jordi slowly.'

Despite LSC being a head final language, LSC gaps forward, thus realizing an SOV-SO order. As we saw in 4.2.1 for Farsi and Turkish, LSC goes against Ross's (1967) generalization, which considers

\footnotetext{
${ }^{28}$ Johnson (2014), instead, assumes that gapping only involves the verb and no other elements, on the basis of the presence of the No embedding constraint (Hankamer 1979). Neijt (1979) supports the same view.
} 
gapping forward in head final languages impossible ${ }^{29}$. Moreover, since the gap is present only in the second conjunct, Johnson's (2014) generalization of gapping that states that the verb can only be missing from the second conjunct in order to be gapping is respected. Furthermore, Right Node Raising (RNR), which would result in a SO-SOV order, is not allowed, as in (301b), in contrast to gapping in (301a).

(301) a. MARINA COFFEE PAY, JORDI CROISSANT

PAY. (39.25)

'Marina paid for a coffee and Jordi for a chocolate.'

b. *MARINA COFFEE PAY, JORDI CROISSANT PAY.

As for the types of verbs that can appear in gapping, LSC shows no restrictions, differently than other SLs such as HKSL. In (302) we can see that plain verbs (302a), agreement verbs (302b) and verbal CL (302c) are allowed in gapping. Moreover, in (302a') we can see that even with plain verbs that need an auxiliary like LOVE, it is possible to mark agreement through the use of NMMs like body and head shift/lean. Tang \& Lau (2012) report that in HKSL, instead, it is not possible to realize gapping with verbal CL or agreement verbs, as we saw in 4.2.3. For these last two types, we will see more examples when looking at the properties of $\varphi$-features mismatch in gapping in 5.5.1.

${ }^{29}$ In 5.6 we will further discuss this issue putting it in relation also with the directionality of coordination. 
(302) a. IX-1 STONE LIKE MARINA FLOWER. (66.1.03) 르

'I like stones and Marina flowers.'

$\underline{\mathrm{hl}+\mathrm{bl}+\mathrm{bs} \text { ips. }}$ hl+bl contral.

a'. MARINA JORDI LOVE MARC JORDINA. (56.02)

'Marina loves Jordi and Marc, Jordina.

b. FRIEND IX-3-ARC FLOWER 3-CL:GIVE-1 JORDI WATCH

3-CL:GIVE-1. (56.14) 는

'Some friends gave me flowers and Jordi a watch.'

c. MARINA BALL BASKETBALL CL:PICK-UP JORDI GOLF BALL. (71.14)哭

'Marina picked up basketball balls and Jordi the golf balls.'

As for the syntactic properties that characterize gapping, in the literature review in 4.2.1 we saw that the most common in English to look at are cross-conjunct binding, the sensitivity to the No embedding constraint and therefore whether gapping can appear only in coordination or also in subordination, island sensitivity and scope of negation and modals. Due to the lack of sufficient research on quantifiers on LSC, it will not be possible to look at cross-conjunct binding in LSC, but we will focus on the other aspects.

In English, gapping is considered to be a special type of ellipsis. Gapping in English respects the No embedding constraint, it is sensitive to islands and it can only appear in coordination. According to the no embedding constraint, the gapped verb cannot be embedded (Hankamer 1979), as shown in (303a), repeated from (241a) in 4.2.1. Moreover, the antecedent of the gapped verb cannot be embedded either (303b), where the sentence is taken (somewhat 
awkwardly) to be a conjunction of two clauses and not a single one with an embedded coordination.

(303) a. *Alfonse stole the emeralds, and I think that Mugsy stole the pearls.

b. *She's said Peter has eaten his peas, and Sally has eaten her green beans, so now we can have dessert.

(Hankamer 1979: 19, 20)

This principle is only obeyed by gapping, though, and no other type of ellipsis is affected by it. VPE is an example of that, as in (304).

(304) Mary ate a sandwich and I think that Mike did, too.

In LSC, though, as we also saw for Farsi (Farudi 2013), the gapped verb can be embedded, as illustrated in (305a). Differently from Farsi, though, the antecedent in LSC cannot occur in an embedded clause (305b).

(305) a. JORDI DOUGHNUT EAT, MARINA SAY IX- 3 j MARC j $_{\text {CROISSANT }}$ EAT. (55.01)

'*Jordi ate a doughnut and Marina said that Marc a croissant.'

b. *MARINA SAY JORDI DOUGHNUT EAT, MARC CROISSANT EAT.

Similarly to gapping, the No embedding constraint does not apply in VPE, as in English (306). 
(306) MARINA DOUGHNUT EAT CAN MARC SAY JORDI CAN EAT ALSO. (85.01)

'Jordi can eat a doughnut and Marina said that Marc can, too.'

For Farsi, Farudi (2013) connects the possibility of gapping to appear in embedding contexts to movement. Looking at island sensitivity, she argues that the locality conditions on movement are relevant in constraining where the gap can be present. As we can see in (307), repeated from (244) in 4.2.1, both in Farsi and in English, gapping is sensitive to islands. This is also the case in LSC (308).

(307) a. *Giti mardhā-yi ke futbāl bāzi mi-kon-an

(Farsi)

Giti men-IND that soccer play IMPFV-do-PL

dust dār-e va Sārā mardhāyi ke tenis dust dār-e.

like have-SG and Sara men who tennis like have-SG

‘ Giti likes men who play soccer and Sarah likes men who tennis.'

(Farudi 2013: 72)

b. *Suzy doesn’t like men who play instruments, and Mary, sports.

(Coppock 2001: 12)

(308) *MARINA [rel MAN SAME ${ }^{30}$ HOUSE BUILD] LIKE, JORDINA [rel MAN SAME SWIMMING POOL BUILD] LIKE.

'*Marina likes men who build houses and Jordina likes men whe build swimming pools.'

${ }^{30}$ SAME is used to mark the presence of a relative clause in LSC (Mosella 2012). 
As for the type of structure that can license gapping, in English it can be found only in coordination, differently from other types of ellipsis. (309) illustrates the ungrammaticality of gapping in clausal adjuncts (309a) and the opposite for VPE (309b), as underlined in Coppock (2001).

(309) a. *John will have caviar, although others beans.

b. John will have caviar, although others won't.

(Coppock 2001: 3)

Differently from English, gapping in LSC can also be found in clausal adjuncts (310a), as it is the case also for VPE (310b). This follows from the violation of the No embedding constraint.

a. MARINA FRUIT EAT BECAUSE JORDI CAKE EAT. (49.09)

'Marina ate fruits because Jordi ate some cake.'

b. MARINA FRUIT EAT CAN BECAUSE JORDI FRUIT EAT CAN ALSO.

'Marina can eat fruits because Jordi can, too.'

Till now, gapping and VPE in LSC turn out to share the basic properties that distinguish them in English. As we can see in the summary in Table 2, gapping in LSC does not seem to be a special type of ellipsis as it is in English. Therefore, gapping in LSC is not English-like and can be put in the same category with the other types of ellipsis. 


\begin{tabular}{|c|c|c|c|}
\hline & & Gapping & VPE \\
\hline \multirow[t]{2}{*}{ English } & $\begin{array}{l}\text { no embedding } \\
\text { constraint }\end{array}$ & Applies & Does not apply \\
\hline & $\begin{array}{l}\text { exclusive to } \\
\text { coordination }\end{array}$ & Yes & No \\
\hline \multirow[t]{2}{*}{ LSC } & $\begin{array}{l}\text { no embedding } \\
\text { constraint }\end{array}$ & Does not apply & Does not apply \\
\hline & $\begin{array}{l}\text { exclusive to } \\
\text { coordination }\end{array}$ & No & No \\
\hline
\end{tabular}

Table 2: Properties of gapping and VPE in English and LSC.

Based on the properties we saw till now, gapping in LSC, though, can be recognized if it follows the following description in (311).

\section{(311) Gapping in LSC}

In a structure $\mathrm{A} c \mathrm{~B}$, where $\mathrm{c}$ represents the presence of coordination or clausal adjunction, gapping deletes a string in $\mathrm{B}$ that is identical to a string in $\mathrm{A}$ in which at least the verb is present, optionally together with other elements such as arguments of the verb or adjuncts. Moreover, in B, more than one remnant are left undeleted. The remnants need to be in a parallel relation of contrast with their correlate antecedents.

As for the scope of negation and modals, in 4.2.1 we saw that in gapping in English these two elements can either have wide or distributed scope over the two conjuncts. In a sentence like the one in (312), repeated from (248) in 4.2.1, the scope is ambiguous. 
(312) a. John can't eat caviar and Mary, beans.

b. Wide: $\neg[$ can [ John eat caviar and Mary eat beans ] ]

c. Distributed: $\neg$ [can [ John eat caviar]] \& $\neg$ [ can [ Mary eat beans ]]

(adapted from Siegel 1987: 7-9)

Winkler (2005), though, notices that in order to have wide scope it is necessary to have a specific context, and she supports a denial analysis for wide scope looking at prosody. Wide scope, in fact, is licensed by the presence of a peak on the remnants but also on the negation that is absent for the distributed one, as we can see in the example in (313) repeated from (250) in 4.2.1.

(313) a. Wide scope:

Leon CAN'T eat CAVIAR and Anna BEANS.

$\left(\mathrm{H}^{*}\right) \quad \mathrm{H}^{*}+\mathrm{L} \quad \mathrm{H}^{*}+\mathrm{L} \mathrm{H} \quad\left(\mathrm{H}^{*}\right) \mathrm{H}^{*}+\mathrm{L} \mathrm{H} \%$

b. Distributed scope:

The LEFT eye can't go UP and the OTHER one DOWN. $\begin{array}{llll}\mathrm{H}+\mathrm{L}^{*} & \mathrm{H}^{*}+\mathrm{L} \% & \mathrm{H}^{*}+\mathrm{L} & \mathrm{H}^{*} \mathrm{LL} \%\end{array}$

(Winkler 2005: 200, 201)

For LSC we saw the first data on scope of negation in 3.2.3.2 when we presented the tests to identify coordination in LSC. The data showed that in LSC a single negation cannot scope over two conjuncts. As we can see in (314), in order to negate both conjuncts, it is necessary to overtly negate both conjuncts, adding for example a negative element such as ALSO-NOT (either) in the second conjunct. 
(314) a. *TEACHER FOOD COOK SANDWICH BUY NOT.

Ungrammatical if meaning 'The teacher didn't cook the food and (didn't) buy a sandwich.'

Grammatical if meaning 'The teacher cooked some food and didn't buy a sandwich.'

b. GIORGIA WINE DRINK LIKE-NOT JORDI BEER LIKE ALSO-NOT. (75.26)

'Giorgia doesn't like drinking wine and Jordi doesn't like beer either.'

Comparing ALSO-NOT to the NPI tampoco 'either' in Spanish, it is possible to see some differences. Camacho (2003) describes the behavior of tampoco in Spanish in relation to word order. As we can see in $(315 \mathrm{a}, \mathrm{b})$, when it is in a postverbal position, tampoco is licensed by negation, while it cannot be preceded by negation if it is preverbal (315c).

(315) a. Marta no vino tampoco.

(Spanish)

b. *Marta tampoco no vino.

c. Tampoco Marta vino.

'Marta didn't come either.'

(Camacho 2003: 22 adapted from Bosque 1980)

While in Spanish it is possible to have overt negation in the sentence with the postverbal NPI tampoco, this is not the case for the LSC negative ALSO-NOT, as we can see in (316). In (316a) there was a previous negative context given. ALSO-NOT then, does not necessarily 
look like an NPI in LSC but rather like an element carrying negation itself.

a. JORDI COME ALSO-NOT. (75.27)

'Jordi didn't come either.'

b. GIORGIA WINE DRINK LIKE-NOT JORDI BEER LIKE-(*NOT)

ALSO-NOT. (75.26) 를

'Giorgia doesn't like drinking wine and Jordi *(doesn't)

like beer either.'

Going back to the structure of negation for LSC proposed by Quer (2002) that we saw in (285) and that is repeated here in (317), having ALSO-NOT so high in the structure means that it is in SpecNegP and either it does not lexicalize the head of NegP or it is in complementary distribution with the negative sign NOT.

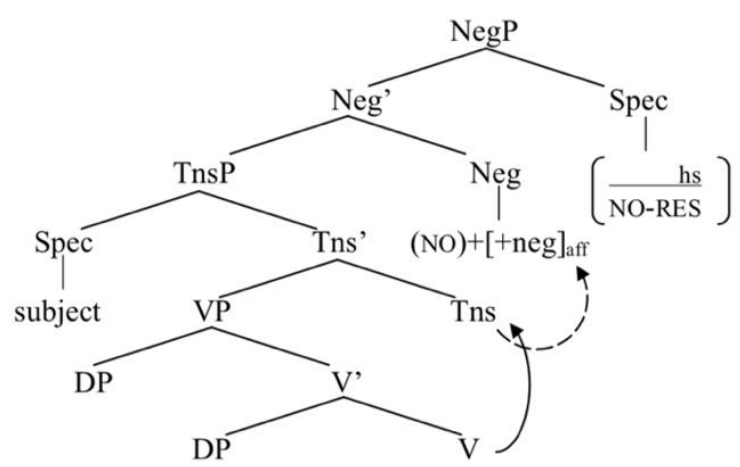

(Quer 2002)

In gapping in LSC, though, differently than cases of full TP/CP coordination, it is possible to negate both conjuncts, supporting a distributed reading of negation. As we can see in (318), the negation 
can be interpreted in both conjuncts. Trying to elicit wide scope negation in gapping through denial, instead, makes ALSO-NOT appear in the second conjunct, as in (319), thus making actual wide scope of negation absent. Even though there are accentuated NMMs on the negation in (319) expressing denial, it is anyway necessary to have a negative element like ALSO-NOT in the second conjunct.

MARINA CHOCOLATE LIKE-NOT JORDI CHEESE. (54.12)

'Marina doesn't like chocolate and Jordi (doesn't like) cheese.'

(319) A: YESTERDAY MARINA JORDI SEE. MARINA T-SHIRT BUY JORDI SHOE.

B: IMPOSSIBLE! IX-1 SEE MARINA JORDI. IX-3 $3_{i}$ MARINA $_{i}$ T-SHIRT BUY NOT JORDI SHOE *(ALSO-NOT)! (54.13) 党

A: 'Yesterday I saw Marina and Jordi. Marina bought a t-shirt and Jordi a pair of shoes.'

B: 'It's impossible! I saw Marina and Jordi. Marina didn't buy a t-shirt and Jordi a pair of shoes either.'

Since ALSO-NOT carries negation itself like a negative sign, it is reasonable to conclude that wide scope of negation is not available in gapping in LSC. Following Winker (2005) and Repp (2009), it is possible to argue for a default distributed scope of negation in gapping for LSC as well. The same holds if a negated modal is present, as in (320). 

'Marina can't dance and Jordi paint, either.'

Differently than English then, wide scope in LSC is not available in gapping and this will be a crucial point that we will consider later in the analysis for gapping, in which small-conjuncts coordination will be excluded in favor of a large-conjuncts coordination structure (contra Johnson 2009-2014 and others).

We will see next the discourse properties of gapping in LSC that will further support a large-conjuncts coordination analysis.

\subsection{Discourse properties in gapping in LSC}

In 4.2.1 we saw that prosody and information structure play an important role in determining the properties of gapping. Based on Kuno (1976), the remnants in the second conjunct are considered to represent new information. Moreover, for the contrastive principle (Winkler 2005), the remnants must occur in a contrastive relation to their antecedent correlates. In a question-under-discussion approach, as we have already seen in chapter 4 , multiple wh-questions are considered to generate sets of alternatives for each remnant supporting the presence of contrast among them and their correlates. Moreover, based on Kuno (1976), Winker (2005) proposes the contrastive topic and contrastive focus principle for gapping. Looking also at prosodic contour, she claims that the first remnant is a contrastive topic and the second remnant a contrastive focus. Coppock (2001) and Toosarvandani (2013), instead, assume both 
remnants to be focalized. Depending on the type of approach to Information Structure, it is possible to consider focus and contrast as two separate notions (Vallduví \& Vilkuna 1998) or focus as a marker that generates contrasting alternatives (Rooth 1992). For LSC I will follow Winker (2005) in assuming the contrastive topic and contrastive focus principle. Both remnants are considered to be in parallel contrast with their correlates, and the presence of contrastive topic or focus on the subject depends on the context given, even though subjects tend to be topicalized in LSC. As already pointed out, differently than spoken languages, sign languages use NMMs to express prosody (Quer \& Pfau 2010) and, therefore, to also mark the relation between the elements in a sentence and information structure. In the next section we will see how contrastive topic and contrastive focus are marked in gapping in LSC, thus confirming Winkler's principle also for LSC.

\subsubsection{NMMs of contrastive topic and contrastive focus ${ }^{31}$}

Since the first studies on sign languages, Fisher (1975) and Liddle (1980) claimed for ASL that topicalized elements are found in the left periphery of a sentence and are marked by raised eyebrows (Pfau \& Quer 2010). Aarons (1994) identifies other NMMs for topic such as mouth open, backwards movement of the head and eyes wide open. As claimed by Pfau \& Quer (2010), though, for most of SLs, such fine description has not been made yet.

\footnotetext{
${ }^{31}$ Thanks to Alexandra Navarrete-González for feedback on this part.
} 
Moving into contrastive topic, in addition to sentence initial position and the use of NMMs such as raised eyebrows to mark topicalization, signing space is used as well, as claimed by Barberà (2015) for contrastive topics in LSC. In Figure 18, it is possible to see how the horizontal plane is mapped. The ipsilateral side corresponds to the position of the dominant hand of the signer, while the contralateral one to the non-dominant hand. Two signs with the pragmatic function of topics can contrast with each other thanks to the use of the space: they are placed one in the ipsilateral side of the space and the other on the contralateral one.

\section{Ipsilateral Contralateral}

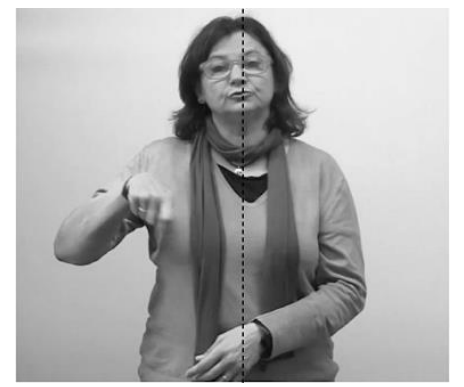

Figure 18: Use of space in LSC, producing the sign for JORDI in contrastive topic.

If we look now at an example of gapping, we can see the distribution of the NMMs and the use of space that have been introduced above. In (321), the subjects MARINA and JORDI, both marked with contrastive topic, show raised eyebrows to mark their nature as topics and they are put in contrast thanks to the head lean towards the ipsilateral side of the space spread on the subject in the second conjunct (see Figure 18). 


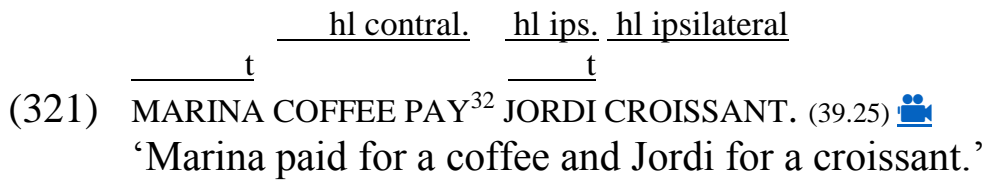

Often, both contrastive topics are marked with head leans (hl) towards opposite sides of the space, as in (322). As in (323), instead, the head leans can be directed towards the same side of the space, but in these cases, there is always contrast expressed in the space thanks to the placing of other elements of the sentence. MARINA is a body anchored sign while JORDI is signed in space. In (323), the verb PAY, one of the signs following the subjects in the first conjunct, is signed in the space and it is placed in the contralateral side. JORDI, therefore, subject of the second conjunct, is signed in the ipsilateral one in order to mark the contrast with MARINA in the first conjunct, even if the head leans on the subjects are both towards the ipsilateral side. This different use of space in each conjunct marks the distinction of the two conjuncts in space and establishes the parallelism between the elements contrasting in each one.

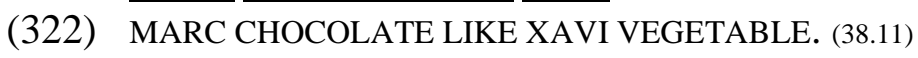

'Marc likes chocolate and Xavi vegetables.'

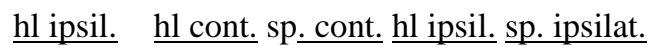

(323) MARINA PIZZA PAY $\frac{\text { re }}{\text { JORDI DESSERT. }(68(+67) .12) \text { 를 }}$
'Marina paid for the pizza and Jordi for the dessert.'

\footnotetext{
${ }^{32}$ Even if not annotated in all the examples discussed, on the verb is very often present a head nod, whose function will be explained in this section.
} 
Very often, head lean is also accompanied by the use of a body lean (bl) towards the same direction of the head. The sign, if not body anchored, can also be found in the same side of the space of the NMMs, like in (324).
sp. ipsil.
$\underline{\mathrm{hl}+\mathrm{bl} \text { ipsil. } \mathrm{hl+bl} \text { ipsil. }}$
sp. contral.
(324)
$\frac{\mathrm{re}}{\text { JOAN }} \frac{\mathrm{re}}{\text { WATCH BUY JORDI }}$ PLANT. (70.02)
'Marina bought a watch and Jordi a plant.'

The main markers of contrastive topic are then raised eyebrows to mark topicalization and the combination of the use of space, head lean and body lean to mark the contrast between the two referents. Sometimes, weak single head nod or reduced head thrust are also found, but it might be given by the articulation of the sign itself. If the sign is body anchored, it is normal to sign it approaching the head towards the hand or that the head follows the articulation of the sign in the opposite direction of the body. Finally, a break whose length and intensity varies is found at the end of each contrastive topic, as also mentioned in Pfau \& Quer (2010) as a marker of intonational phrase.

Contrastive focus, differently than contrastive topic, got more attention in the SL literature. Wilbur (1999) looked into the properties of ASL to mark prominence. Taking sentence-final position as the default position where to find prominence in a language, some languages like English, in order to put in focus a non-sentence final element, do not need to affect the syntax, they just move the stress from the default sentence-final position to the element of interest. 
These languages are described as having plasticity. Other languages, which lack this characteristic, like Catalan, do not move the stress, and they affect the word order of the sentence, by moving the element to focalize to the sentential final position (Vallduví 1990). As for ASL, as described by Wilbur (1999), "ASL prefers word order determined by discourse relations and does not allow prominence plasticity". LSC, instead, seems to adopt both strategies. In LSC, in constructions like VP ellipsis, it is possible to move the prominence on different elements of the sentence depending on discourse properties. Like in English, the peak accent moves to the relevant element and the same happens in LSC. Raised eyebrows (re) and head forward (hf) are used to mark a focalized element in a sentence, like in (325a) on the subjects and in (325b) on the object. These markers have been identified as markers of focus also in other SLs, as we will see below. In (325b) the NMMs of prominence on FRUIT are way more marked compared to the raised eyebrow used on the subjects.

bl ipsi.

re+hf re+hf

(325) a. MARINA FRUIT 3-GIVE-1 JORDI 3-AUX-1 ALSO. (68(+67).10) 'Marina gave me some fruit and Jordi did, too.' bl ipsil. bl contral.

b. $\frac{\text { re }}{\text { MARINA }} \frac{\text { re+hf }}{\text { FRUIT } 3-G I V E-1} \frac{\text { re }}{\text { JORDI 1-AUX-1 ALSO. }(68(+67) .11)}$ 'Marina gave me some fruit and Jordi did, too.' 
Navarrete-González (2016), though, notices that in LSC it is also possible to affect the word order in order to focalize an element, as we can see in (326).

(326) a. 'What do you eat?'

b. EAT $[B R E A D]_{F}$

'(I) eat bread.'

(Navarrete-González 2016: 25)

The data in (325) and (326) show that in LSC it is possible to express focus moving the peak accent but also affecting word order. The status of LSC in relation to plasticity, though, needs more research.

Contrastive focus has been described for several SLs, including ASL, NGT and LSF. The main marking of focus found for ASL are raised eyebrows, forward leans (Wilbur and Patschke 1998; Wilbur 2012; Schlenker et al. 2016), longer hold time and greater sign amplitude. As for contrastive focus, Schlenker et al. (2016) found that, both in ASL and LSF, it is marked by raised eyebrows, accompanied also with forward leans in ASL, and with head nods and greater sign amplitude in LSF. In NGT, Crasborn and van der Kooij (2013) identify, as markers of focus, raised eyebrows and eye gaze, while contrast in contrastive focus is marked using contrast in space and body leans towards left and right (van der Kooij et al. 2006).

If we look into the expression of contrastive focus in gapping, LSC shows very similar NMMs present in other SLs (confirmed also by Navarrete-González 2016). Repeating example (321) here as (327), we can see that COFFEE and CROISSANT, marked with 
contrastive focus, show head lean towards opposite sides of the space. Despite the fact that PAY is not contrasting with any element in the sentence, head lean spreads over it. I would like to argue that it is due to assimilation of movement for articulatory reasons. In Figure 19, we can see a picture of the sign for CROISSANT in (327).

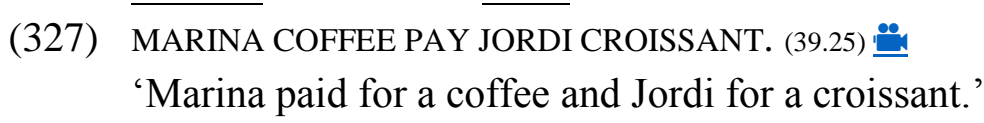

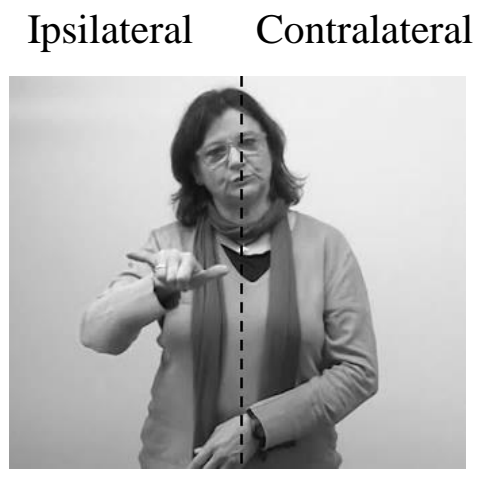

Figure 19: Use of space in LSC, producing the sign for CROISSANT in contrastive focus.

As we saw before for contrastive topics, the movement of the head towards the same side of the space in both conjuncts can be compensated for. When the two objects are body anchored signs, a contrastive use of space in placing other elements of the sentence can be used. (328) is an example, where COFFEE and DESSERT are both body anchored signs and marked with head lean towards the ipsilateral side of the space. The verb in the first conjunct is signed in the ipsilateral side of the space, while the subject of the second conjunct in the contralateral one. As mentioned above, marking in 
space each conjunct assures the parallelism between the elements contrasting in each one.

hl ipsil. h. nod $\underline{\text { s. cont. }}$

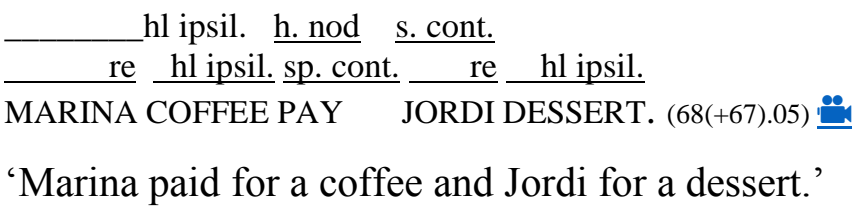

When having two objects that are both non body-anchored signs, instead, the contrast in space is always expressed, and accompanied by head and in case also body leans, as in (329).

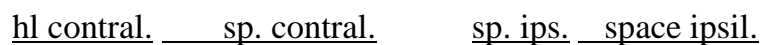
re hl contral. h.nod re hl ipsil.

MARINA SWISS CHARD PAY JORDI POTATO. (68(+67).07)

'Marina paid for Swiss chard and Jordi for potatoes.'

Very often, though, the object in the first conjunct is also marked with raised eyebrows, as found also in LSF as marker of contrastive focus. Moreover, there are body leans other than only head leans. On the object of the second conjunct, mainly head leans and contrast in space are found. A different distribution of NMMs where, for instance, there is absence of contrast in space or head leans, would result in an ungrammatical sentence.

The same NMMs of contrastive focus are also found on the subject of each conjunct in VPE. As claimed for spoken languages, Frazier et al. (2007) argues for English that both subjects are accented due to the contrast between them, showing contrastive focus. Moreover, the presence of contrastive focus is confirmed by the fact 
that in VPE, for both subjects, there is a closed set of alternatives. A sentence like the one in (330) can answer the question "Who gave me some fruit?", where the wh-element creates those sets. In that example we can see that the contrast between the two subjects is again marked by head lean towards opposite sides of the signing space, produced in combination with body shift (bs) and raised eyebrows (re) that mark the presence of focus.

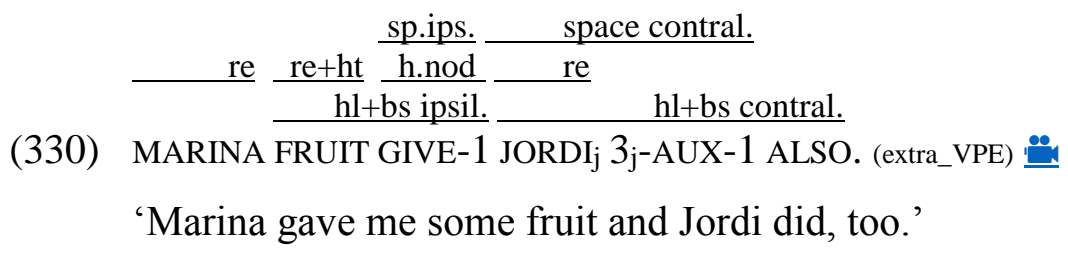

As markers of contrastive focus it is also possible to find head lean, systematically present to mark contrast, body lean or shift, contrast in the use of space and raised eyebrows. These markings, which we also listed above for other SLs, can be claimed to be used crosslinguistically.

It is important to notice, though, that in LSC these markings correspond to the same ones found for contrastive topic, as also noticed by Navarrete-González (2018). As pointed out to me by Elena Koulidobrova (p.c.), it is also possible to consider that the NMMs found only indicate the presence of contrastive focus, which then must also involve topicalization. In this case, focus would be considered to generate sets of alternatives, which then it is possible to topicalize. In this work, though, I am considering contrastive focus and contrastive topic as both generating alternatives thanks to contrast itself. Therefore, I claim that in LSC there is no clear 
distinction between marking contrastive topic and contrastive focus, mainly due to the fact that focalized elements often show raised eyebrows in contexts other than sentence initial position, where they are usually interpreted as topics. In order to distinguish the type of contrast on a specific element, contextual information, information structure and discourse properties can tell us whether it is a contrastive topic or a contrastive focus. As pointed out in Wilbur (2012), referring to Molnar and Winkler (2010), the difference between contrastive topic and contrastive focus, if contrast is understood as an "information structural notion", is that focus is used as a highlighting device and topic as having a linking function to elements previously introduced in the discourse.

Finally, in the expression of gapping it is important to notice that the given material, the verb in the first conjunct, is always characterized by specific NMMs as well: a deep head nod. The same NMMs are found on the given elements in VPE, as in example (330), where not just the verb but also the given direct object in the first conjunct is marked with a head nod. So far, head nod in the literature has been considered a mark of existence in sentences where no copular sign is expressed (Liddell 1980, Aarons 1994) or as a marker of focus (Wilbur 1991). Liddell (1980), referring to a case of gapping in ASL, claims that the head nod found on the remnant objects is again a marker of existence. Nobody yet, though, looked at the distribution of NMMs on the given elements in gapping and VPE. Therefore, I claim that, in LSC, head nod can be also a marker of giveness, namely of an element that has been already previously introduced in the context. As for spoken languages (Selkirk et al. 
2008), discourse-given material is not prosodically prominent, but it can be distinguished anyway from other types of focus, such as contrastive or new information (see Destress Given by Féry and Samek-Lodovici 2006 for discussion about this). For LSC, it is then possible to claim that no discourse stress is found on the given material, but the fact that it is given is marked through the use of a deep head nod.

There seems to be a pretty clear parallelism between English and LSC in the prosodic marking of gapping and VPE that make Winkler's (2005) analysis of contrastive topic and contrastive focus cross-linguistic and cross-modal. It is the case also for VPE, if we follow Frazier et al. (2007), who assume the presence of contrastive focus on the subject of each conjunct in VPE. Finally, having a deep head nod as a marker of givenness in LSC shows another crosslinguistic and cross-modal parallel with spoken languages where given material is also prosodically marked (Féry and SamekLodovici 2006).

Lastly, the examples presented in this section show that also gapping in LSC respects the contrastive topic and contrastive focus principle (Winker 2005), as in English. This suggests the need for both languages to provide a proper syntactic representation of information structure and therefore support that a large coordination structure must be present, involving the left periphery of the sentence for each conjunct. Following Pfau \& Steinbach (2008), it is possible to confirm that also for SLs the arguments of the verb that get topicalized need to move to TopP. For focus movement, instead, I will adopt Merchant's (2004) assumption related to the remnant left 
in fragment answers. The analysis proposed by Merchant (2004) for fragment answers, in fact, assumes that there is movement plus deletion. The movement is a focus movement of the remnant to an agnostic position FP, even though Merchant points out that it might be Rizzi's (1997) FocP, as we can see in (331). This movement of the remnant to FocP can also be assumed for LSC.

(331) a. Who did she see?

b. [FP [DP Johni] [TP-she saw $\left.\left.\mathrm{t}_{\mathrm{i}}\right]\right]$.

(adapted from Merchant 2004: 673)

We will see next the arguments that will further shape the analysis for gapping in LSC, based on the conclusion that gapping in LSC needs a large-conjunct coordination structure in order to provide an adequate syntactic structure to account for discourse properties.

\subsection{Towards an analysis of gapping in LSC}

In the previous sections we presented the properties of gapping also in relation to other types of ellipsis showing that gapping in LSC does not present all the characteristics that are typical of gapping in English: it is sensitive to islands, like in English, but the No embedding constraint does not apply and it can appear also in adjunct structures, differently than English. Moreover, wide scope of negation (and modals) cannot be expressed, therefore there is no ambiguity of this element, again differently than English. Despite these syntactic differences, LSC and English share the same 
discourse properties, though, supporting the presence of an active left periphery.

In the following sections we will enter the analysis of gapping in LSC, reconsidering the data that exclude the presence of smallconjunct coordination and therefore the possibility of adopting Johnson's (2009-2014) analysis. The analysis proposed will support a unified deletion at PF account for gapping and VPE that can be potentially extended to other types of ellipsis in LSC, as suggested by Coppock (2001) for English.

\subsubsection{Against small-conjunct coordination and ATB}

As we saw in 4.2.2.1, several accounts propose to analyze gapping assuming the presence of small-conjunct coordination, among which Johnson's (2009-2014) is one of the most popular. The main arguments used to support this view are the presence of wide scope of negation and modals and cross-conjuncts binding. Moreover, Johnson (2009-2014) points at the differences in the properties of gapping as being the sole elliptical structure appearing only in coordination to support a unique account for gapping in opposition to other elliptical constructions. One of the main arguments used by Johnson to justify low coordination concerns the presence of wide scope of negation and modals, which supports the need of having $\mathrm{vP}$ coordination under TP, as in examples (332) and (333) repeated from (256) and (257) in 4.2.2.1. 
(332) Ward hasn't prepared natto and his guest kumquats.

(Johnson 2004: 64)

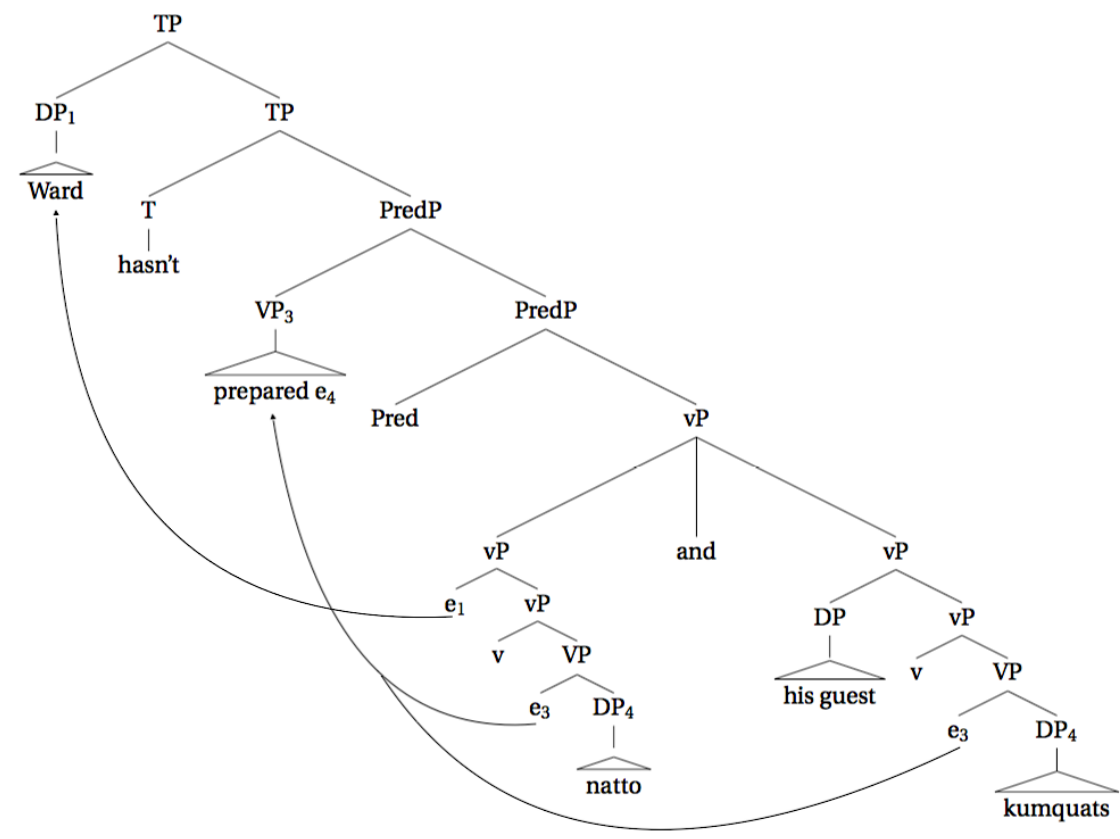

As we saw in 5.2, though, in LSC wide scope of negation and modals is not available. In order to have a wide scope reading of negation it is necessary to have a denial interpretation of the sentence, as claimed by Winkler (2005). We can see an example for LSC in (334), repeated from (319) in this chapter. The presence of ALSO-NOT in the second conjunct shows the necessity of always having negation interpreted in both conjuncts and therefore the absence of wide scope. In (335), repeated from (318) in this chapter, we can see an example of distributed scope of negation in gapping as the default reading. As pointed out by Winker (2005) and Repp (2009), in fact, the distributed scope is the default reading of negation in gapping. 
A: YESTERDAY MARINA JORDI SEE. MARINA T-SHIRT BUY JORDI SHOE.

B: IMPOSSIBLE! IX-1 SEE MARINA JORDI. IX-3 i MARINA $_{i}$ T-SHIRT BUY NOT JORDI SHOE *(ALSO-NOT)! (54.13)

A: 'Yesterday I saw Marina and Jordi. Marina bought a t-shirt and Jordi a pair of shoes.'

B: 'It's impossible! I saw Marina and Jordi. Marina didn't buy a t-shirt and Jordi a pair of shoes either.'

MARINA CHOCOLATE LIKE-NOT JORDI CHEESE. (54.12)

'Marina doesn't like chocolate and Jordi (doesn't like) cheese.'

The absence of wide scope of negation in the expression of denial in LSC rules out the potential parallelism between English and LSC in the need of having low coordination.

Discourse properties support the same perspective due to the need of giving a proper syntactic structure that accounts for the presence of contrastive topic and contrastive focus on the remnants and their corresponding antecedents. Winkler (2005) accounts for the contrastive relation between the remnants proposing that they move to an A-bar position inside vP (see also López \& Winkler 2002). In LSC, though, if we assume the movement of the verb to $\mathrm{T}$ in the case of agreement verbs and the presence of material in $\mathrm{T}$ in case of plain ones, it is not possible to adopt Winkler's (2005) analysis. Moreover, the interaction of the verb with negation gives further support to the presence of large-conjunct coordination in LSC. As we saw in 5.1.1, NMMs of negation can spread over the verb in the absence of an overt 
negation like NOT, suggesting that the verb moves to the head of NegP. The presence of $\mathrm{V}$ to $\mathrm{T}$ movement that can further extend to Neg confirms again the large-coordination status of coordination in gapping in LSC and discourse properties underline the need of having $\mathrm{CP}$ coordination, as projections containing TopP and FocP.

Let us see next the arguments that will help justify the presence of a deletion at PF account for gapping in LSC and also for VPE.

\subsubsection{Deletion}

As we saw in 4.2.2, several authors propose an analysis for gapping that involves PF-deletion of VP or TP, assuming the presence of structure in the ellipsis site, as in Merchant (2001). Merchant (2001) claims that the material that is missing needs to be in a relation of identity with the antecedent and therefore it needs to be given to respect the e-GIVENness condition (see also 4.1.2.2). Coppock (2001) follows this analysis for sluicing and VPE and she applies it to gapping, as we saw in 4.2.1. Moreover, Gengel (2006) and Farudi (2013) add the need to account syntactically for the presence of contrastive topic and contrastive focus by moving the arguments of the verb to the left periphery, even if they consider the movement to TopP and FocP only of the remnants in the second conjunct. As for the part of the structure that gets deleted, they both argue for the deletion of the whole TP left empty of non-given material. 
Let us review the arguments that support the claim of having structure present in the elliptical site in gapping in LSC that will justify deletion.

\subsubsection{Evidence for structure in gapping}

There are several pieces of evidence that support the existence of structure in the ellipsis site: island sensitivity, case matching effects, complementizer deletion, distribution of infinitivals, predicate answers and intermediate reconstruction effect in sluicing. Due to the absence of morphological case, prepositions, overt complementizers like "that" and infinitivals, in LSC it is only possible to test island violation. For gapping, Coppock (2001) looked at "propositional islands" following Merchant (2001) to show that remnants are actually A'-moving. Example (336), repeated from example (213) in 4.1.1.2, is ungrammatical because there is a violation of the relative clause island.

(336) *Suzy doesn't like men who play instruments, and Mary, sports.

(Coppock 2001: 12)

The ungrammaticality of the sentence in (336) shows that the presence of structure in the ellipsis site is detected and that the remnants are A'-moved. In LSC, as in English, gapping is sensitive to propositional islands. Following Coppock (2001), we can see that also in LSC there is structure in the elliptical site that gets detected 
and makes the sentence ungrammatical, as in (337), repeated from (308).

*MARINA [rel MAN SAME HOUSE BUILD] LIKE, JORDINA [rel MAN SAME SWIMMING POOL BUILD] LIKE.

'*Marina likes men who build houses and Jordina likes men who build swimming pools.'

The sentence in (337) also shows that LSC displays similar properties to Farsi in relation to movement. In 4.2, we reported that for Farsi, Farudi (2013) examines island sensitivity and connects to movement the possibility of gapping to appear in embedded contexts, hence without following the No embedding constraint. She argues that the locality conditions on movement are relevant in constraining where the gap can be present. As we just saw in 4.2, gapping in LSC can also appear in embedded contexts and, as presented in (337), it is sensitive to islands. Therefore, for LSC it is possible to follow Farudi (2013) in assuming that the possibility of having gapping in an embedded context is related to the possibility of movement.

Going back to the data to justify the presence of material in the gapping site, in 4.1.2.2 we saw that Culicover and Jackendoff (2005: 273), though, point out examples that show that island violation is possible in gapping, as we can see in (338) repeated from (214) in chapter 4.

(338) Robin knows a lot reasons why dogs are good pets, and Leslie, cats. 
In LSC it is possible to express the same meaning, but the structure does not show the same characteristics. As we can see in (339), the clausal complement needs, for example, to be extraposed.

(339)

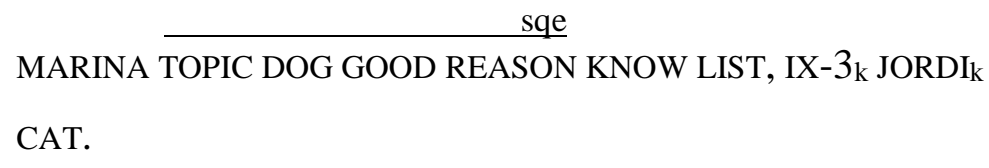

'Marina knows a lot reasons why dogs are good pets, and Jordi, cats.'

The island sensitivity of gapping in relative clauses in (337) justifies, then, the adoption of deletion at PF for gapping in LSC. The detection of structure in the elliptical site goes against the non-structural views. Moreover, as we will see in 5.5, the deletion account at PF, which assumes the presence of identity between the conjuncts, will be further justified. The next concern is to justify that the part of the structure deleted in gapping is the whole TP.

\subsubsection{What can you delete?}

In the previous sections in this chapter we saw several arguments that support the presence of large-conjunct coordination in gapping in LSC: i) absence of wide scope of negation and modals, ii) $\mathrm{V}$ to $\mathrm{T}$ movement of the verb with agreement verbs and anyway the activation of T with plain verbs through the use of AUX or of NMMs, and iii) presence of contrastive topic and contrastive focus on the remnants. The last property, following Gengel (2006) and Farudi (2013), suggests the need of having movement of the remnants to the 
left periphery, therefore to TopP and FocP. As specified in 5.3.1, the NMMs used to express contrastive topic and contrastive focus in gapping and contrastive focus in VPE are very similar, and the previous context or discourse properties determine the presence of one or the other. Their being contrastive, anyway, determines the presence of set of alternatives for each remnant. Merchant's (2001) e-GIVENness condition, extended to gapping by Coppock (2001), then, can be also applied to LSC gapping. For the sentence in (340), repeated from (261) in 4.2.2.1, Coppock (2001) presents the focus closure of the gapped clause and of the antecedent clause, as we can see in (341). Being the two identical, they entail one another and therefore deletion is licensed.

(340) $\left[\alpha \mathrm{John}_{\mathrm{F}}\right.$ likes caviarF $]$ and $[\gamma$ MaryF beansf $]$.

(341) a. F-clo $(\alpha)=\exists x \exists y[x$ likes $y]$

b. F-clo $(\gamma)=\exists x \exists y[x$ likes $y]$

(Coppock 2001: 7)

Assuming that focus closure is given by the presence of contrast, either on topicalized or focalized elements, in LSC it is also possible to have the same representation suggested by Coppock (2001), as we can see in (342) and (343).

(342) $[\alpha$ FRIENDCT FLOWERCF 3 -GIVE-1] [ $\gamma$ JORDICT WATCHCF].

'Some friends gave me flowers and Jordi a watch.'

(343) a. F-clo $(\alpha)=\exists x \exists y[x$ 3-GIVE-1 y]

b. F-clo $(\gamma)=\exists x \exists y[x$ 3-GIVE-1 y] 
The authors that support the presence of deletion in ellipsis assume that the syntax in the ellipsis site is considered to be the same of its antecedent, but it gets deleted thanks to a feature that indicates that the phonological value of the constituent targeted is null. This feature is called E-feature, standing for Ellipsis-feature (following Merchant (2001) we find van Craenenbroeck (2010), Aelbrecht (2009), van Craenenbroeck and Lipták (2006), Toosarvandani (2008), Toosarvandani (2009), Vicente (2006), Corver and van Koppen (2010, 2011), and Ha (2008)). The E-feature has a syntax, a semantic and a phonology since it keeps all the information about the ellipsis site. In Merchant (2001), this feature is added to the "licensing" head indicating that all the material present in its sister gets deleted. Gengel (2006), instead, places it in the head of the higher projection that gets deleted. For LSC, I will follow Merchant (2001) in placing the F-feature in the sister of the projection that will get deleted, together with the part of the structure below it.

In the syntactic structure that can account for gapping in LSC, it is necessary to consider, first of all, two crucial aspects: the need of satisfying discourse properties by moving the remnants to TopP and FocP in the left periphery and $\mathrm{V}$ to $\mathrm{T}$ movement of agreement verbs and activation of $\mathrm{T}$ with plain verbs, as we can see in the structure in (345) for the sentence in (344).

space+hl+bl ipsl. space contral. re re+hl cont. hl ipsil.

(344) $\overline{\text { FRIEND IX-3-ARC FLOWER 3-CL:GIVE-1 JORDI WATCH }}$

3-CL:GIVE-1 (56.14)

'Some friends gave me flowers and Jordi a watch.' 


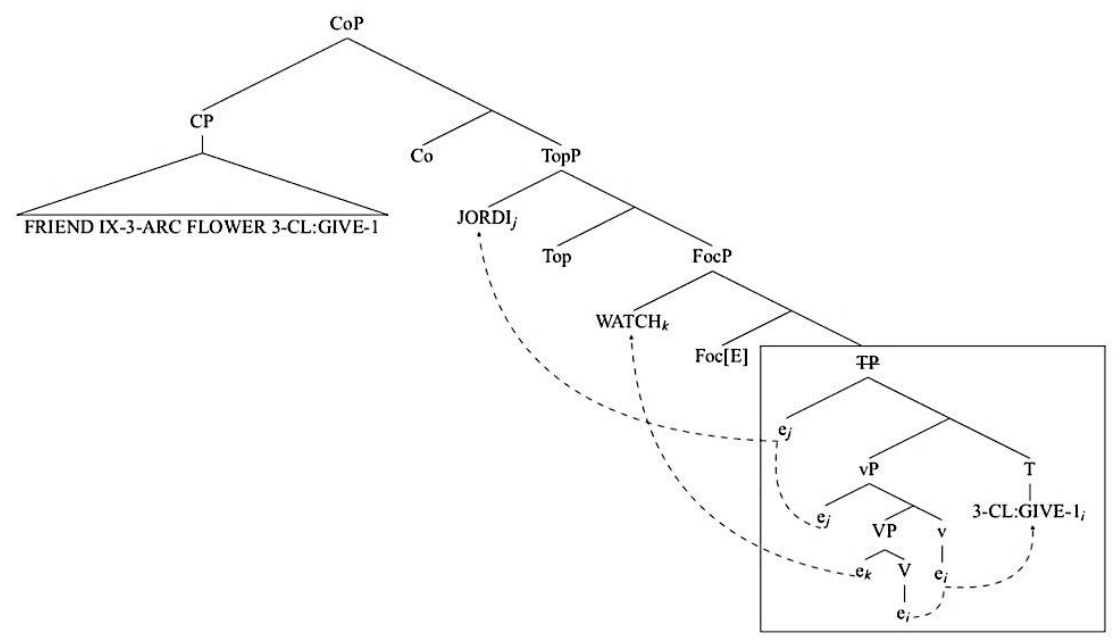

Evacuating the two remnants, the whole TP remains empty of nongiven material and therefore it can be deleted. The E-feature is then placed in the head of FocP. As for the first conjunct, as far as I know, the is no literature that addresses the issue of its structure in particular. As we saw till now, though, the NMMs found on the remnants of the second conjunct are also found on the correlate antecedents in the first one. Moreover, as analyzed by Winkler (2005), in spoken languages the remnants and the correlate antecedents are also marked phonologically, as we can see in (346).

(346) Leon CAN'T eat CAVIAR and Anna BEANS.
$\left(\mathrm{H}^{*}\right) \quad \mathrm{H}^{*}+\mathrm{L} \quad \mathrm{H}^{*}+\mathrm{L} \mathrm{H} \quad\left(\mathrm{H}^{*}\right) \mathrm{H}^{*}+\mathrm{L} \mathrm{H} \%$

(Winkler 2005: 200)

In (345), then, also the elements in the first conjunct move to TopP and FocP in order to respect a parallelism between the first and the second conjunct. 
As we saw in 4.2, gapping and VPE ellipsis show many properties, among which two crucial ones that make gapping in English special with respect to VPE: in LSC, both structures do not respect the No embedding constraint and they can both appear in clausal adjuncts. As proposed for VPE in English (Merchant 2001), in LSC it is possible to assume the presence of deletion and apply the E-GIVENness condition assuming identity between the antecedent and the second conjunct (see 4.1.2.2). Moreover, as we saw in 5.3.1, the remnant in VPE is marked with contrastive focus, as also found for English by Frazier et al. (2007). In order to account for discourse properties, the remnant in the second conjunct needs to move to FocP in the left periphery and in order to respect the parallelism between the two conjuncts, also the subject in the first conjunct needs to move to FocP. Due to the presence of the auxiliary in T for both English and LSC, following Pfau et al. (2018) for the position of the auxiliary in SLs, the [E] feature will be placed in the head of $\mathrm{T}$ in order to have the whole vP deleted. In (348) we can see the representation of the syntactic structure of the VPE example in (347).

(347) [C. FOCUS MARINA] FRUIT GIVE GIVE-1 [C. FOCUS JORDI ${ }_{j}$ ] FRUIF GIVE 3-AUX-1 ALSO.

'Marina gave me some fruit and Jordi did, too.' 


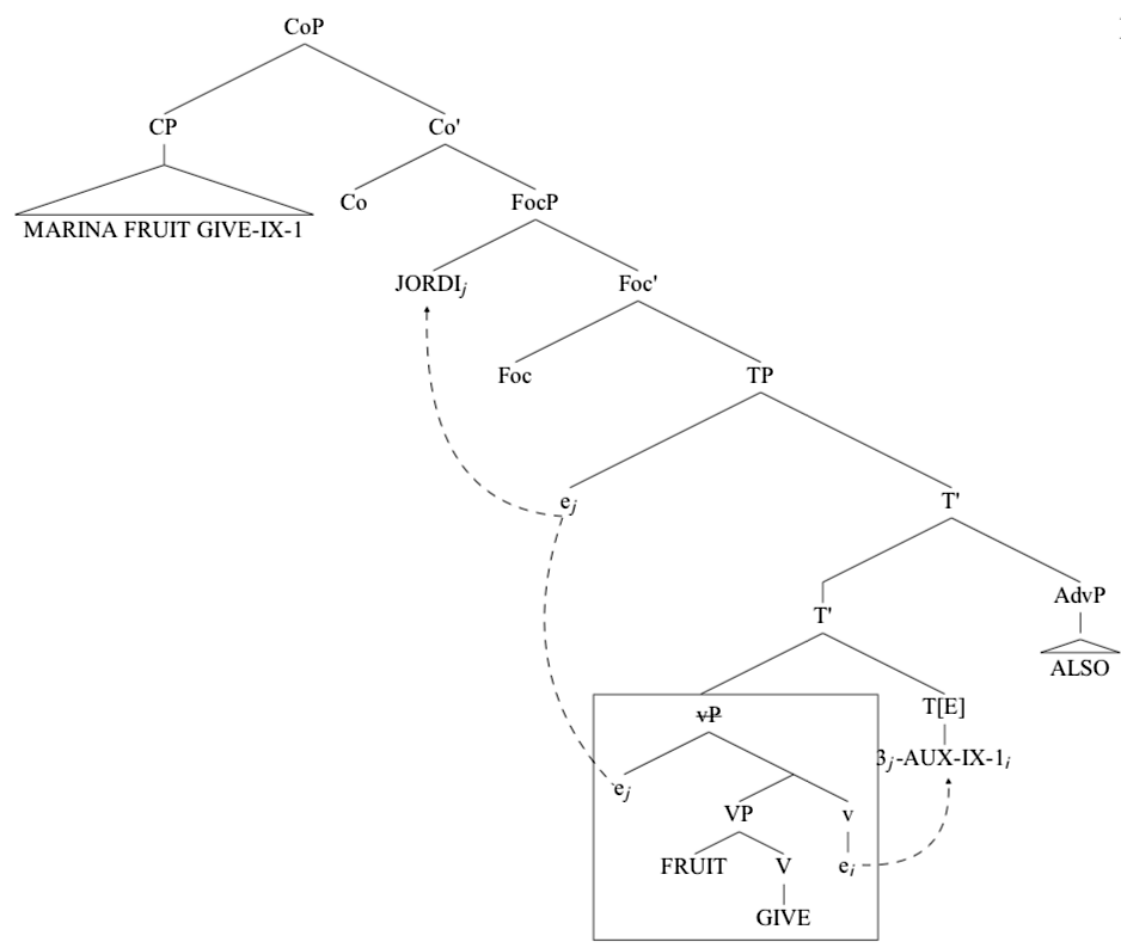

As proposed by Coppock (2001) for English, the LSC facts can be explained under a unified deletion account for both gapping and VPE. In the two structures, though, the part of the structure that gets deleted is different, TP for gapping and $\mathrm{vP}$ for VPE respectively.

In the classification of the different types of ellipsis in $5.1 \mathrm{we}$ pointed out that in LSC, differently than LIS, VPE is found only when an element placed in T like an auxiliary or a modal is left as remnant together with the subject of the verb in the second conjunct, as in (349a). If there is only one remnant left, optionally together with a focus particle, this would be a case of stripping, as in (349b). 
(349) a. MARINA FRUIT 3-GIVE-1 JORDI 3-AUX-1

VPE

FRUTT 3-GIVE-1 ALSO. (extra_VPE)

'Marina gave me some fruits and Jordi did, too.'

b. MARINA CHOCOLATE EAT CAN JORDI ALSO. stripping

'Marina can eat chocolate and Jordi, too.' (68(+67).20)

For VPE it is possible to confirm that the part of the structure that gets deleted is the whole $\mathrm{vP}$; for stripping, though, assuming $\mathrm{V}$ to $\mathrm{T}$ movement or anyway the activation of $\mathrm{T}$, what gets deleted is the whole TP (assuming the movement to FocP of the remnant subject). It is important to underline, then, that stripping and gapping, even though the same portion of the structure gets deleted, are different. In gapping the verb is removed in the second conjunct, possibly together with other elements, but it is crucial that the remnants are contrasting with correlate antecedents. For stripping, this is not the case. Gapping in LSC, then, is not fully English-like but it can be attested as a phenomenon different than other types of ellipsis.

Let us look next at the identity condition in gapping, another important aspect of ellipsis that looks at the recoverability of the material the gets deleted.

\subsection{Identity in gapping}

In ellipsis, an important question to address concerns the recoverability of the content of the ellipsis site, also referred to as identity condition. In the literature, the identity condition is claimed to be due either to semantics or to morphosyntax. In the first case, the 
material in the antecedent and in the elliptical site have the same meaning, while in the second case, it is the form that is responsible for identity. As we saw in 4.1.2, syntactic identity is justified by the presence of voice mismatch (Merchant 2012), morphological mismatch (Lasnik 1995 and Posdam 1997) and vehicle change (Fiengo \& May 1994), even though Merchant (2012) uses this as an argument for semantic identity. As for semantic identity, Merchant (2001) underlines the importance to respect the e-GIVENness condition, on the basis of Rooth (1992) and Schwarzchild (1999). For SLs, Cecchetto et al. (2015), in order to support the presence of syntactic identity in VPE in LIS, apply some tests that are SLmodality specific, namely adverb incorporation, presence of strict and sloppy reading in interaction with Role Shift (RS) and vehicle change. For gapping, though, no tests on identity have been proposed so far for SLs. In the next sections we will look at $\Phi$-feature mismatch in gapping in LSC and we will adapt Cecchetto et al.'s (2015) test on adverb incorporation in order to support the presence of both syntactic and semantic identity in gapping in LSC. As for semantic identity, the presence of the e-GIVENness condition applied by Merchant (2001) on the basis of Rooth (1992) and Schwarzchild (1999) was already justified in 5.3 and 5.4.

\subsubsection{Identity in form: $\varphi$-features mismatch}

In the analysis of the identity relation between an elided phrase and its antecedent, several cases of feature mismatches have been identified in ellipsis, also involving $\varphi$-features. In the resolution of 
ellipsis, $\varphi$-features have been commonly considered irrelevant for the identity condition (Merchant 2006), contrary to categorial and selectional ones. For ASL and LSF, Schlenker (2014) analyses two specific feature types on loci (locations in signing space corresponding to discourse referents) as $\varphi$-features in spoken languages because they remain unspecified in ellipsis constructions. The ones he considers are [plural] and [high]. The former is used to agree with a plural argument and the latter with referents whose height is relevant in the context and marked in the signing space for the verb to agree with. In this section we will describe the $\varphi$-feature mismatch in gapping in LSC, considering also classifier (CL) constructions, in order to draw a cross-modality parallelism for identity in form (syntactic). Moreover, following Bošković (2008), I will provide a classification for the types of $\varphi$-features that can undergo mismatch under ellipsis in LSC.

As we saw in 5.1.1, recent accounts of agreement in SLs follow a minimalist approach (Pfau et al. 2017; Costello 2016) and they assume that in SL there is material that moves to $\mathrm{T}$ via feature checking, in a different way depending on the verb class.

In order to look at $\varphi$-features mismatch in gapping only agreement verbs will be relevant since they are the only ones showing overt morphology (PATH) to agree with the arguments.

The $\varphi$-features considered in SLs, when looking at agreement, are [number], [person] and [location]. Costello (2016), though, collapses the last two in the [identity] feature because it allows to distinguish one referent from another. We can see an example in (350) and (351) of the derivation of an agreement verb that agrees 
with both subject and object in LSE. We can see that the verb moves from $\mathrm{V}$ to $\mathrm{v}$ till $\mathrm{T}$ checking [number] and [identity] features, that include both [+person] and [+location] features, as we already showed in 5.1.1.

(350) IX $_{\mathrm{x}} \quad \mathrm{xTRICK}_{1}$.

'He's tricking me.'

(Costello 2016: 291)

(351)

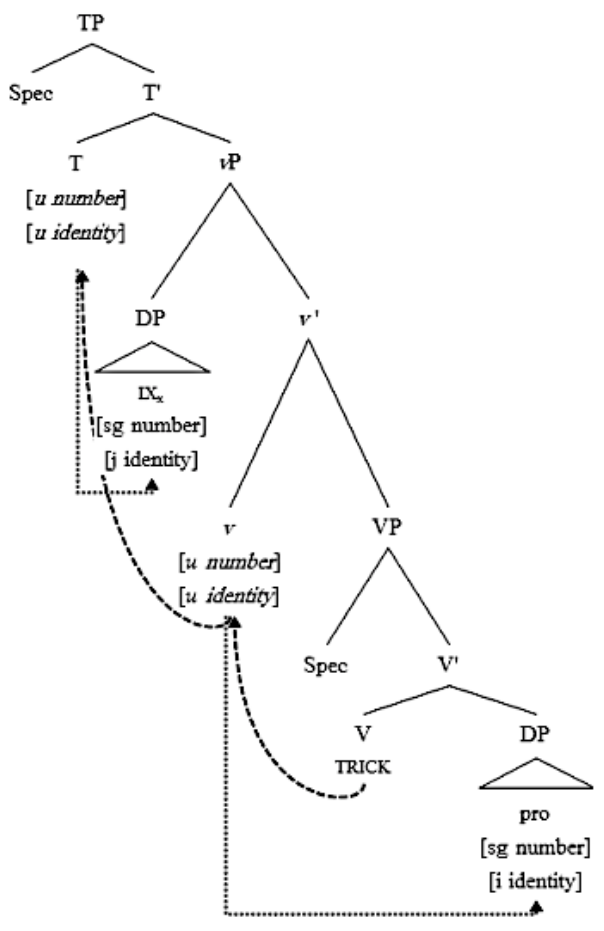

In turn, Schlenker (2014) specifies the presence of [plural] and [high] in ASL and LSF to enter the derivation as $\varphi$-features. They are considered so because they are not interpreted in ellipsis resolution, as we can see in (352) for ASL. 
(352) Context: Tomorrow there is a swimming competition. A team of six French swimmers competes against a single German swimmer.

IX-ARC-a 6 FRENCH SWIMMER LIKE PEOPLE SUPPORT IX-ARC-a. IX-B GERMAN SWIMMER SAME- a,b.

'The six French swimmers like people who support them. The German swimmer does, too.'

(Schlenker 2014: 314)

Barberà (2016) confirms the presence of these two features also in LSC and she argues that, on the same plane, also specificity is marked in space through the activation of a high area of the signing space that marks lack of specificity. The feature [high], then, marks hierarchical position, locative information and non-specificity (Barberà 2014). As assumed by Schlenker (2014), [high] is in contrast with [low] or [normal], depending on the context. It is important to specify that [high] is realized on the vertical plane of the signing space, while [plural] can be expressed mainly on the horizontal one. This distinction explains the possible combination of the feature [high] with [plural], as in the case of 'many birds', for example, where the sign for 'birds' is realized in a high position on the vertical plane and it is reduplicated on the horizontal one. I will use [plural], as opposed to [singular], counting that these two are further specifications of the feature [number].

Other features are claimed to be markers of agreement when using verbal classifiers (CL) in SLs. As defined by Zwitserlood (2012), "classifiers are generally considered to be morphemes with a 
non-specific meaning, which are expressed by particular configurations of the manual articulator and which represent entities by denoting salient characteristics". There are different types of CL that can be used as verbal CL adding movement to them, according to the classification by Benedicto \& Brentari (2004): a) whole entity, where the handshape stands for a whole entity that can also be an instrument (instrumental CL); b) handling, where the handshape represents the way of holding a referent, and c) body part, where the hand refers to a part of the body. We can see an example for each type of CL in LSC in (353).

(353) a. Whole-entity $C L$

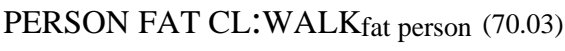

'A fat person is walking.'

a'. Instrumental $C L$

MARINA VEGETABLE (FORK) CL:EAT fork

'Marina ate the vegetables with a fork.'

b. Handling $C L$

MARINA BOTTLE CL:GIVE-1 $1_{\text {bottle (70.04) }}$

(LSC)

'Marina gave me a bottle.'

c. Body part CL

HEAD JOAN WALL CL:HIThead-wall (70.17)

(LSC)

'Joan hit the head on the wall.'

For the first two types, Glück and Pfau (1997), looking at German Sign Language (DGS), argue that they are agreement verbs. Meir (1999), instead, for Israeli Sign Language, claims them to be cases of 
noun incorporation. The two main tests used to verify the presence of noun incorporation, also in spoken languages, are modifier stranding and doubling.

As for the first one, in many spoken languages that allow noun incorporation in the verb (Mohawk, Caddo from Rosen (1988) and Mohawk form Baker (2009)), we can see the use of stranding modifiers. The modifier (adjectives, numerals and demonstratives) appears outside of the verbal construction, but it refers to the incorporated noun (354). In (355) we can see an example from Israeli Sign Language (ISL) from Meir (1999). The adjective NEW is modifying the CL inside the verbal CL. I think it is important to notice, though, that there is a pronoun after the adjective that might suggest that this is what the adjective is modifying and not necessarily the CL in the verbal CL.

(354) Kanekwarúnyu wa?-k-akya? tawi? tsher-ú:ni. (Mohawk) 3N.dotted.DIST PAST-1sg.3N-dress-make

'I made a polka-dotted dress.'

(Rosen 1989: 299)

(355) NEW IX-3 a aGIVE-CL:C

new that give-cylindrical-object-me

'Give me the new cup (over there).'

(Meir 1999: 305)

As for doubling, the languages that allow stranding a modifier also allow doubling. It is possible to have an overt NP outside the verbal construction that refers to the incorporated noun. The NP outside 
usually refers to a more specific entity in the set designated by the noun incorporated, as in (356) for Mohawk. The same happens also in ISL, as in (357). The NP CUP is more specific than the CL in the verbal CL that refers to a class of objects of cylindrical shape.

(356) Tohka niyohserá:ke tsi nahe? sha? té:ku

(Mohawk) several so.it.numbers so.it.goes eight nikú:ti rabahnót wahu-tsy-ahní:n ki rake? níha. of.them bullhead 3M.3N-fish-bought this my-father 'Several years ago, my father bought eight bullheads.'

(Mithun 1984: 870)

(357) PLEASE CUP INDEX a aHAND-CL:C. please cup that cylindrical-object-hand-me 'Please hand this cup to me.'

(Meir 1999: 304)

Considering both diagnostics, modifier stranding cannot apply to LSC, as we can see in the ungrammatical examples in (358a', b', c', d), as also in other SLs like DGS (Glück \& Pfau 1997). It is not possible to modify the verbal CL just having a modifier appearing before it, an overt NP needs to be outside the verbal construction, close to the modifier. As for doubling, the pattern in LSC is similar to the one described by Rosen (1989). It matches also the data presented for DGS, that is, the CL is less specific than the NP it classifies. While Rosen (1998) claims that doubling is optional, this is not the case for LSC, nor for DGS (358e). It is always necessary to 
have the NP that the CL is referring to, expressed outside of the VP. In LSC, the absence of the overt NP outside the verbal CL does not make the sentence ungrammatical but it does not necessarily mean, as in the case of (356b), that it was a bottle that was given to the signer, as it is the case in (359a). In (359c) there is an example for DGS that presents similar data. Only with instrumental CL doubling it is not necessary, but in this case, the form of the verbal CL is exactly the same of the one of the NP that it is referring to. Anyway, verbal CLs with an instrumental CL inside cannot be considered cases of noun incorporation because the NP the verbal CL is referring to cannot be modified if doubling is not present, as in (358d, d').

'Marina saw a fat person walking.'

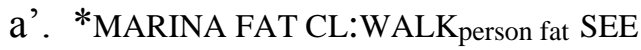

'Marina saw a fat person walking.'

b. MARINA BOTTLE BIG CL:GIVE- $1_{\text {bottle }}(70.06)$

'Marina gave me a big bottle.'

b'. *MARINA BIG CL:GIVE- $1_{\text {bottle }}$

'Marina gave me a big bottle/a vase/....'

c. JOAN HEAD BIG head CL:HIThead-wall (71.05)

'Joan hit his big head on the wall.'

c'. *JOAN BIG WALL CL:HIThead-wall

'Joan hit his big head on the wall.'

d. MARINA VEGETABLE FORK BIG CL:EAT fork-big (70.20)

'Marina ate vegetables with a big fork.' 


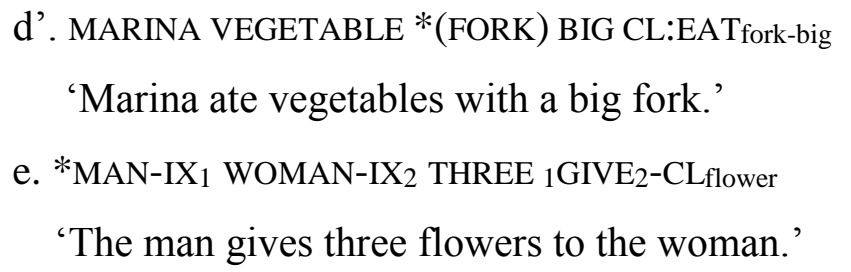

(Glück \& Pfau 1997: 4)

a. MARINA BOTTLE CL:GIVE- $1_{\text {bottle }}$ (70.04)

'Marina gave me a bottle.'

a'. MARINA CL:GIVE- $1_{\text {bottle }}$

'Marina gave me a bottle/a vase/...'.

b. MAN-IX 1 WOMAN-IX 2 *(FLOWER $)_{1}{ }_{1} \mathrm{GIVE}_{2}-\mathrm{CL}_{\mathrm{a}}$

(DGS)

'The man gives a flower to the woman.'

(Glück \& Pfau 1997: 6)

On the basis of this data, it is possible to conclude that whole-entity $C L$, instrumental $C L$, handling $C L$ and body parts $C L$ are not cases of NP incorporation as a lexical formation process.

The other analysis proposed for CL is the syntactic one, and, as Glück and Pfau (1997) point out, there are two possible ways to go: a) word formation by head incorporation (Baker, 1988) or b) word formation by assignment of features. Let us go through both of them.

With respect to option a), Baker (1988) describes subject/object incorporation in the verb in Mohawk, where incorporation implies that the head of the NP moves into the verb leaving a trace behind. Therefore, subject incorporation is 
impossible, since the trace would not be governed. Object incorporation, though, is possible, as in (360).

(360) a. Yao-wir-a?a ye-nuhwe?-s ne ka-nuhs-a? PRE-baby-SUF 3fs/3n-like-ASP DET PRE-house- SUF 'The baby likes the house.'

b. Yao-wir-a?a ye-nuhs-nuhwe?-s PRE-baby-SUF 3fs/3n-house-like-ASP 'The baby house-likes.' c. *Ye-wir-nuhwe?-s ne ka-nuhs-a? 3fs/3n-baby-like- ASP DET PRE-house-SUF 'Baby-like the house.'

(Baker 1988: 83)

Glück and Pfau (1997) claim that, in DGS, this analysis does not work because it is not possible to assume that the noun leaves a trace behind if an overt NP outside the verbal CL is required. Moreover, the NP itself would not be a copy of the incorporated element since the $\mathrm{CL}$ is less specific, as it is possible to see in (361), adapted from (359a, a'), where the CL refers to a class of objects with the shape similar to the one of a bottle. In LSC it is the same as in DGS (361b). Moreover, both in DGS and in LSC it is possible to have a CL referring to the subject, while it is not allowed in spoken languages (Baker 1988). (361c) is an example.

(361) a. MARINA *(BOTTLE) GIVE- $1_{\text {bottle }}$

'Marina gave me a bottle.' 
b. MAN-IX 1 WOMAN-IX 2 *(FLOWER $)_{1}$ 1GIVE$_{2}-\mathrm{CL}_{\mathrm{a}}$

(DGS)

'The man gives a flower to the woman.'

(Glück \& Pfau 1997: 6)

c. PERSON FAT CL:WALK person fat

'A fat person was walking.'

As for the second option b), Glück and Pfau (1997) analyze CLs as an instance of agreement. The main argument they use to prove this claim is the use of left dislocation and the presence of a resumptive pronoun (following Lillo-Martin 1991). In ASL, with plain verbs, when having left dislocation either of the object or of the subject, it is necessary to have a resumptive pronoun. With agreement verbs, though, it is optional (362). The same happens in DGS, as reported by Glück and Pfau (1997).

(362) a. Topicalized subject of a plain verb

(ASL)

$\overline{\text { BROTHER }}_{1}$, JULIE2 THINK *(PRONOUN 1 ) BRUSH-THEETH FINISH.

'My brother, ${ }_{i}$ Julie thinks he ${ }_{i}$ already brushed his teeth.'

b. Topicalized subject of an agreement verb $\mathrm{t}$

BROTHER $_{1}$, JULIE2 THINK *(PRONOUN 1$)$ 1LOOK-OVER3 CAR3 FINISH.

'My brotheri, Julie thinks (he $e_{i}$ ) already looked over the car'

c. Topicalized object of a plain verb

$\overline{\text { COOKIE }_{1}}$, PRONOUN $_{2}$ HOPE SISTER 3 SUCCEED 3PERSUADE4

MOTHER4 EAT * $\left(\right.$ PRONOUN $\left._{1}\right)$.

'That cookie $\mathrm{i}$, I hope my sister manages to persuade my mother to eat it ${ }_{\mathrm{i} .}$ ' 
d. Topicalized object of an agreement verb

$\overline{\text { MAN }}_{1}$, STEVE 2 SAY JULIE
FINISH 3GIVE1 (PRONOUN
) BOOK.
'That $\operatorname{man}_{\mathrm{i}}$, Steve said Julie already gave a book to (him ${ }_{\mathrm{i}}$ '

Moreover, when having verbal CLs, the resumptive pronoun is not required and this proves CLs to be marking agreement (363).

(363) a. $\overline{\text { GLASS }_{\mathrm{a}}-\mathrm{IX}_{1}}$, CHILD THINK, MAN (IX-3 1$)$ TABLE $_{2}$

(DGS) 2TAKE-CLa.

'The glass $i$, the child thinks, the man takes it ${ }_{i}$ off the table.'

b. $\frac{\text { PENCIL }}{\text { PIX }_{1}}$, CHILD THINK, (IX-3 $)_{1}$ HILL $_{2}{ }_{2}$ ROLL-CL $_{a}$.

'This pencil $l_{i}$, the child thinks ( $\mathrm{it}_{\mathrm{i}}$ ) is rolling down the hill.'

(Glück \& Pfau 1997: 12, 13)

In LSC, this distinction between plain and agreement verbs, based on the use of a resumptive pronoun to refer to a topicalized element, is not so neat (for more discussion on this see Quer \& Rosselló 2013). In (364), (365), (366), it seems that both plain and agreement verbs do not need a resumptive pronoun to refer to the subject or the object topicalized. In the case of the object, there is no difference when it is topicalized in plain (364) or agreement verbs (365).

(364) a. Topicalized object of a plain verb, signed in the space, [inanimate]

CAR ${ }_{\mathrm{j}}$, MARINA THINK JORDI IX-3i BUY (IX- $\left.3_{\mathrm{j}}\right) .(71.07)$ ien

'The car, Marina thinks Jordin bought.' 
a'. Topicalized object of a plain verb, body anchored, [inanimate]

FRUIT $\mathrm{j}$, MARINA THINK JORDI BUY (IX-3 $\left.3_{\mathrm{j}}\right) .(71.11)$

'Some fruit, Marina thinks Jordi bought'.

b. Topicalized object of a plain verb, signed in the space, [animate]

JORDIj, PROFESSOR THINK IX-3 $3_{\mathrm{i}}$ MARC $\mathrm{i}_{\mathrm{i}}$ KNOW (IX- $\mathrm{3}_{\mathrm{j}}$ ). (85.04) 'Jordi $\mathrm{i}_{\mathrm{i}}$, the professor thinks Marc knows himi.'

(365) a. Topicalized object of an agreement verb, signed in the space, [inanimate]

CARj, MARINA REMEMBER SEE (IX- $3_{\mathrm{j}}$ ). (71.12)

'The car, Marina remembered seeing.'

a'. Topicalized object of an agreement verb, body anchored, [inanimate]

FRUITj, MARINA REMEMBER SEE (IX- $\left.3_{\mathrm{j}}\right) .(71.13)$ 를

'Some fruit, Marina remembered seeing.'

b. Topicalized object of an agreement verb, signed in the space, [animate]

JORDI $j_{\mathrm{j}}$, PROFESSOR THINK MARC SEE (IX-3 $\left.3_{\mathrm{j}}\right) .(85.05)$

'Jordi $i_{i}$, the professor thinks Marc knows himi.

As for the subject, if it is [inanimate], either with a plain or an agreement verb, the resumptive pronoun is not necessary, as in (366a', c'). It seems obligatory only when the subject is [animate] with plain and agreement verbs (366a, b, c). 
(366) a. Topicalized subject of a plain verb, signed in the space, [animate]

JOAN $\mathrm{j}$, JORDI THINK IX-3 $3_{\mathrm{j}}$ THEETH-BRUSH. (70.08)

'Joan, Jordi thinks he brushed his teeth'.

a'. Topicalized subject of a plain verb, signed in the space, [inanimate]

CARj, MARINA THINK (IX-3 3 ) WORK NOT. (71.08)

'The car, Marina thinks it doesn't work.'

b. Topicalized subject of a plain verb, body anchored, [animate]

WAITER $_{\mathrm{j}}$, MARINA THINK IX-3j MOVIE WATCH. (71.06)

'The waiter, Marina thinks he watched a movie.'

c. Topicalized subject of an agreement verb, signed in the space, [animate]

WAITER, MARINA THINK IX-3j SCHOOL GO. (71.09)

'The waiter, Marina thinks he went to school.'

c'. Topicalized subject of an agreement verb, signed in the space, [inanimate]

CAR , MARINA THINK (IX-3 $3_{j}$ ) BREAK. (85.06) 單

'The car, Marina thinks it's broken.'

In LSC, then, this test does not allow to determine whether CLs are similar to agreement or plain verbs. The analysis in which CLs are considered cases of noun incorporation, though, cannot be applied to verbal CLs: i) it is not possible to modify the NP inside the verbal CL without having doubling; ii) word formation by head incorporation (Baker 1988) is also excluded. As pointed out by Glück and Pfau 
(1997) for DGS, for LSC it is also impossible to assume that the noun leaves a trace behind if an overt NP outside the verbal CL is required. Therefore, I assume that verbal CLs in LSC, as in DGS, are agreement verbs.

Being agreement verbs, verbal CLs also show the need of checking $\varphi$-features. As for the main ones involved in verbal CLs, I argue that they can be labeled [size/shape], since the handshape of the verbal CL can give information about the dimension and the shape of the referents of the arguments it agrees with.

Following Schlenker's (2014: 300) diagnostic, if there is feature mismatch in ellipsis, we can confirm that the ones involved are $\varphi$-features. It is important, then, as diagnostic also in LSC, to look at gapping and the behavior of $\varphi$-features in its resolution. As we saw before for ellipsis in LSF, in LSC there are instances of feature mismatch between the elided phrase and its antecedent. In LSC, then, there are $\varphi$-features that remain unspecified in ellipsis resolution as well.

Before getting into $\varphi$-feature mismatch in gapping in LSC, let us first look more closely at the characteristics of these $\varphi$-features in the language. First of all, it is possible to divide them in two groups, the ones that show obligatory agreement and the ones that show optional one. In (367), $\varphi$-features are expressed obligatorily, while in (368) they occur optionally. The $\varphi$-features in (367) are overtly marked on the NP agreeing with the verb and they are necessarily expressed on the verb, too. The ones in (368), instead, are not overtly marked on the NP agreeing with the verb and they are not necessarily expressed on the verb. With verbal CLs, agreement is considered 
optional because it is also possible to agree with the object using the lexical form of the verb, even though it might be a plain verb. In the case of (368c), the lexical form would be the agreement verb PICKUP and in (368d) the plain verb, EAT. It is important to say that instrumental CL do not refer to a direct object but to an indirect one.

(367) Obligatory agreement.

a. [plural]/[singular], as specifications of [number]

With the subject.

PERSON+++ ORIGIN CATALAN PARLIAMENT GO[plural]. (72.01)

'Catalan people went to the parliament'

a'. With the object/ directional argument.

MARINA CLASS THREE GO+++[plural]. (74.01)

'Marina attended three classes.'

b. [person] (+/- animate)

With the subject/object/indirect object/directional argument.

JOAN j JORDIi WATCH jGIVEi. (74.02) 單

'Joan gave Jordi a watch.'

b'. MARINA PLANT IX-3POSS TAKE-CAREj. (74.03)

'Marina takes care of her plant.'

c. [location] for different referents referring to an actual location signed on the horizontal plane

With a directional argument.

IX-1 HOMEj GOj. (74.05)

'I went home.'

d. [high $] /[$ low/normal $]$ for location

MARINA BIRD SKY [high location] LOOK[high 1.]. (74.06) I는

'Marina looks at the birds in the sky'. 
e. $[$ high $] /[$ low/normal] for specificity

WHO^SOME[high] BIKE[normal] STEAL $\left[\right.$ high]. ${ }^{33}$ (74.08)

'Someone stole the bike.'

(368) Optional agreement.

a. [high $] /[$ low/normal] for hierarchy

BOSS MONEY [high hierarchy] GIVE-1 $1_{\text {[normal]. (74.09) }}$

'The boss gave me money.'

a'. BOSS MONEY [normal]GIVE-1 [normal]. (74.10)

b. [high $] /[$ low/normal] for iconicity

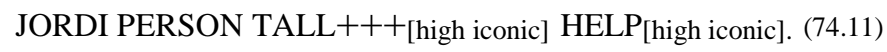

'Jordi helps short people.'

b'. JORDI PERSON TALL+++[high iconic] $\operatorname{HELP}_{\text {[normal]. (74.12) }}$

c. [size/shape] (with handling CL)

MARINA BALL BASKETBALL CL:PICK-UP[size/shape]+++. (74.13)

'Marina picked up basketball balls.'

c'. MARINA BALL BASKETBALL PICK-UP+++. (74.14)

d. [size/shape] (with instrumental CL)

MARINA VEGETABLE CL:EAT[size/shape]. (74.15)

'Marina ate vegetables (with a fork).'

d'. MARINA VEGETABLE EAT. (74.16)

'Marina ate vegetables.'

In (367a), plural agreement with the subject through the use of double-handed $m$-handshape can be realized if the number of referents is high; it is not clear how many referents needs to be

\footnotetext{
${ }^{33}$ This sentence was repeated from Barberà \& Cabredo Hofherr (2017: 8).
} 
involved, though. One of the deaf experts I have been working with can accept to have ten referents, but this judgment is not shared by the other informant. For GO to be modified for plural agreement with m-handshape, the referents need to be at least more than four. Up to four, the verb can be modified using mandshape for two, three, four referents respectively. I do not want to get into the properties of the realization of the [plural] feature on the subject but I want to specify that [plural] can be realized on this argument, despite some morphological limitations of the verbs. For more discussion on this in SLs, I address the reader to Mathur (2000) and Rathmann \& Mathur (2008). The realization of [plural] on the object, instead, is very productive, probably because it involves a reduplication of the movement and not a change in the morphology of the verb itself.

Moreover, $\varphi$-features, both obligatory and optional, can also interact with each other. Let us look at the case of the use of space as "topographic", where a point in the signing space refers to a point that is placed in a projected map of the real space. In the following example in (369), for instance, the interlocutors are referring to a specific house placed outside Barcelona and the verb GO agrees in the space with a point that refers to a projected map of the real space.

(369) Context: Xavi lives in Collserola, an urban park outside Barcelona.

XAVI HOME IX-3POSS GO [iconic]+[location]. (72.12) 를

'Xavi went home.' 
In this example, the agreement with a directional argument is on the vertical plane and not on the horizontal one, as when no map of the real space was projected. I assume that when "topographic space" is used, there is an interaction between [iconic] and [location] features. They complement each other because the first one is optional while the second one obligatory. In the case where the topographic space is not expressed, [location] is enough to make the sentence grammatical where the verb is agreeing anyway with a directional argument.

There are other cases of interaction of features:

- [size/shape] and [person] (optional and obligatory)

- [high]/[low/normal] for hierarchy and [person] (optional and obligatory)

- [plural] and [location], both obligatory.

These features complement each other, but if the optional one is missing, the sentence would be grammatical and express the same meaning anyway. See example (370a) with [size/shape] and [person]. The first feature is optional while the second one is obligatory. The same is true for the interaction between [high]/[low/normal] for hierarchy and [person], optional and obligatory features, respectively. In the example (370b), if [high]/[low/normal] for hierarchy is not expressed, the relation between the arguments would not vary.

(370) a. JOAN JORDI $_{\mathrm{j}}$ BOOK BIG THICK $3_{\mathrm{i}^{-}}$CL:GIVE- $3_{\mathrm{j}}([$ size/shape])[person]. 'Joan gave Jordi a big and thick book.' (74.17) 
b. BOSS MONEY ([high hierarchy][person] GIVE-1 $1_{\text {([normal])[person]. (74.09) }}$

'The boss gave me money.'

In the case of interaction of two or more obligatory $\varphi$-features, they will all be expressed on the verb, as in the case in (371) of the interaction of [plural] and [location].

(371) PERSON+++ ORIGIN CATALAN PARLIAMENT GO[plural][location].

'Catalan people went to the parliament.' (72.01)

In order to examine the properties of these features when they enter the derivation, I will follow Bošković (2008). But let us first go back a little bit looking at how $\varphi$-features are analyzed in the previous literature. In the minimalist program, Chomsky (2001), and others later (Pesetsky and Torrego 2007), describe $\varphi$-features entering the derivation as un/valued and un/interpretable:

- if the lexical entry enters the derivation with the feature already lexically specified, this feature will be valued, otherwise unvalued.

- if the feature contributes to the semantics of the lexical entry, this feature will be interpretable, otherwise uninterpretable.

In (372) we can see an example of how $\varphi$-feature [gender] is expressed on an adjective agreeing with a noun.

(372) Gossa bonica.

(Catalan)

'Pretty dog.' 
The NP gossa enters the derivation with the feature [gender] as valued interpretable. The NP enters the derivation with the [gender] feature lexically specified and this feature also contributes to the semantics of the NP. The adjective bonica, instead, has the feature [gender] entering as unvalued uninterpretable. It is not lexically specified on the adjective and it is not relevant for the semantics of the entry. In the derivation, the adjective, functioning as a probe, looks for a goal that has the same feature valued in order to realize feature checking. The values on the adjective will then be deleted before spell out, since uninterpretable features cannot enter the semantics.

For Chomsky (2001) it is obligatory to check and delete valued uninterpretable features, so they do not enter semantics. Thus, Chomsky (2001) states the Valuation/Interpretability Biconditional (373).

(373) Valuation/Interpretability Biconditional (Chomsky 2001: 5)

A feature $\mathrm{F}$ is uninterpretable if $\mathrm{F}$ is unvalued.

Pesetsky and Torrego (2007), though, introduce a more flexible system where features are "shared" and not assigned. Moreover, they depart from the Valuation/Interpretability Biconditional and they also assume the possibility of having other combinations of features: (i) uninterpretable but valued, and (ii) interpretable but unvalued.

Bošković (2008), contrary to Chomsky (2001), assumes the possibility of not having to check valued uninterpretable features and excludes the need of checking uninterpretable features in general. 
Bošković (2011) reports cases in Serbo-Croatian (SC) where gender can be considered valued and uninterpretable in nouns because of its arbitrariness. In SC, in most nouns, excluding cases such as "man" or "woman", gender does not affect the interpretation of the noun. We can see an example in (374) where gender is arbitrary in nouns.

(374) a. Zelena kola su kupljena. green.FEM car. FEM are bought. FEM 'The green car was bought.'

b. Zeleno auto je kupljeno. green.NEUT car.NEUT is bought.NEUT c. Zeleni automobil je kupljen. green.MASC car.MASC is bought.MASC

(Bošković 2011: 1)

From the data in (374), Bošković (2011) confirms that gender in SC enters the derivation on NP as valued and uninterpretable. Moreover, referring to gender, he also justifies the need of having valued uninterpretable features deleted without being checked. In the example in (375) we can see an example of conjunct agreement: the two NPs coordinated show different genders and the past participle agrees only with one of the two, the feminine. The neuter gender on the second conjunct, then, will enter the derivation as valued uninterpretable but will not be able to be checked and, therefore, it will need to be deleted without being checked. 
Juče su uništene sve varošice i sva yesterday are destroyed.PL.FEM all towns.FEM and all sela.

villages.NEUT

'All towns and all villages were destroyed yesterday.'

(Bošković 2011: 7)

In Bošković's (2008) analysis, valued uninterpretable features can be optionally checked and they can be deleted anyway. Moreover, only unvalued features can function as probes. Following this analysis for LSC, only the features that obligatorily need to be checked and that are also expressed on the NP enter the derivation as valued and interpretable. Valued, because the NP enters the derivation with the $\varphi$-feature lexically specified and interpretable because they contribute significantly to the semantics of the NP. On the verb, they will be unvalued and uninterpretable. Functioning as probe, they will find the goal on the argument NP.

As for the features expressed optionally, they enter the derivation on the NP as valued and uninterpretable. Uninterpretable, because they do not affect the interpretation of the NP since they are optionally expressed. Since it is the same if they are there or not, for example overtly expressed on a pronoun or a classifier that follows them or on the verb, then they do not affect the interpretation of the NP. They will be valued because the NP enters the derivation with the $\varphi$-feature lexically specified. On the verb, instead, there are two possible scenarios depending if the verb agrees with the NP for that feature or not. In the first case, in which the verb agrees with the NP, 
on the verb the optional features will be marked as unvalued and uninterpretable. The goal's features (NP) can be assigned to the probe's features (VP) via feature checking. On the verb the optional features will be valued uninterpretable, but it will be possible to delete them anyway. In the second case, instead, in which the verb does not agree with the NP for that feature, the verb will simply enter the derivation without the need to check the feature on the NP. Being valued uninterpretable on the NP, the optional feature will just get deleted, without being checked. As for feature checking triggering verb movement to $\mathrm{T}$, the verb will anyway move to $\mathrm{T}$ because it will always have to check the [person] feature, also in the case of inanimate referents, which always enter the derivation on the verb as unvalued.

Summarizing, $\varphi$-features in LSC can be of several types and enter the derivation differently:

- $\quad$ [plural]/[singular], [person], [location] (obligatory agreement) enter the derivation as valued interpretable on the NP that the verb will agree with;

- [high]/[low/normal] for iconicity, hierarchy, specificity and location on the vertical plane and

- $\quad$ [size/shape] enter the derivation as valued uninterpretable on the NP that the verb will agree with.

In (376) and (377) it is possible to see an example of the derivation of each class of features. In (376) the verb functions as probe and the goal is the NP. The goal's features (NP) are assigned to the probe's features (VP), which become valued interpretable. In (377), instead, 
if there is agreement with the verb, the verb functions as probe and the goal is the NP. The goal's features (NP) can be assigned to the probe's features (VP) via feature checking, which become valued uninterpretable. Following Bošković (2011), they can anyway be deleted without being further checked. If there is no agreement on the verb, as in (377ii), the VP will not show any $\varphi$-feature and the it will move anyway to $\mathrm{T}$ because it will need to check the unvalued feature for [person], the obligatory one.

(376) Obligatory agreement.

MARINA CLASS THREE GO+++[plural]

'Marina attended three classes.'

DP: [plural] --> valued interpretable

VP: [plural] --> unvalued uninterpretable

(377) Optional agreement.

i) if there is agreement on the verb:

BOSS MONEY [high hierarchy] GIVE-1 $1_{\text {[normal] }}$

'The boss gave me money and Jordi a plant.'

NP: [high $]^{34}$--> valued uninterpretable

VP: [high] --> unvalued uninterpretable

ii) if there is no agreement on the verb

BOSS MONEY [normal] GIVE-1 $1_{\text {[normal] }}$

'The boss gave me money and Jordi a plant.'

NP: [high] --> valued uninterpretable

VP: [high] --> not specified

\footnotetext{
${ }^{34}$ It is important to point out that the sign BOSS is not signed in a high position in the space, and it is rather the location associated with it which is high.
} 
LSC seems to support Bošković $(2008,2011)$ approach in the necessity of having the possibility to not obligatorily check valued uninterpretable features and therefore to account for features that are optionally expressed on the verb via agreement.

Let us now look into gapping and the behavior of the features we just saw. Considering gapping in this respect is interesting because the presence of an overt NP argument in the second conjunct makes the feature contrast clear between the two conjuncts. Moreover, as suggested by Schlenker (2014: 300), the presence of mismatch for a certain feature classifies it as a $\varphi$-feature. This can be used, then, as a diagnostic.

In English, like in (378), it is possible to see $\varphi$-feature mismatch between the elided site and its antecedent. In order to respect identity, we would expect to have a verb in third person singular in the elided site, but we clearly see a features mismatch (likes vs like). Due to this mismatch, in the resolution of ellipsis in English, $\varphi$-features have been commonly considered irrelevant for the identity condition, contrary to categorial and selectional ones (Merchant 2006).

(378) Mary likes pancakes and her parents like French toast.

In (379) we can see the same also for $\operatorname{LSC}^{35}$. The $\varphi$-features on the elided verb and on the one in the antecedent are different. Despite this fact, the sentences, as in English, are totally grammatical. In (379a), the verb agrees with a plural object in the first conjunct while

\footnotetext{
${ }^{35}$ Thanks to Gemma Barberà for discussing an early version of this data.
} 
with a singular one, in the second. (379b), instead, shows a different agreement in space with respect to the arguments of each verb, whose loci are specific ones for each referent. As for (379c), the difference in location in space concerns locative referents. Following Costello (2016), we could collapse [person] and [location] under the unique feature of [identity]. The data in (379b) and (379c) might suggest, though, the need to keep these two features separated. In (379d, e), [high]/[normal/low] are expressing a difference between the two conjuncts in specificity or location of the referents, with respect to the vertical plane. In (379f), the verb in the first conjunct refers to a tall person, marked with a [high] feature. In the second one, though, the verb is supposed to agree with the [low] feature. Despite this mismatch, the sentence is still grammatical. The same holds for [high]/[/normal/low ] for hierarchy in (379g). Finally, (379h, h') show an example of feature mismatch with a verbal CL, involving [size/shape]. In (379h), in the first conjunct, the form of the verb refers to big objects while, in the second one, to smaller ones. In (379h'), the verbal CL in the first conjunct expresses the way of eating with a fork, while, in the second, the overt verb would mean "eat with hands". With an instrumental CL, like in this last example, and also with a handling CL in (379h), context and world knowledge play and important role in the interpretation of the verb in the second conjunct. It would be odd, in fact, to think to eat an apple using a fork without giving a specific context for it. 
(379) a. [Plural]/[singular] - obligatory agreement

MARINA CLASS THREE GO+++[plural] JORDI WORKSHOP ONE

$G \Theta_{\text {[singular]. (74.19) }}$

'Marina attended three classes and Jordi one workshop.'

a'. PERSON+ WOMAN A-LOT SCHOOL GO[plural] OFFICE MAN A-

LITTLE. (72.05)

'Many women went to school and a few men to work.'

b. [Person] - obligatory agreement

MARINA $_{j}$ JORDI $_{i}$ WATCH jGIVEi MARC $k$ JORDINAy PLANT

kGIVE $E_{y}$ (56.09)

'Marina gave Jordi a watch and Marc Jordina a plant.'

c. [Location] - obligatory agreement

MARINA HOME GO $_{j}$ JORDI SWIMMING-POOL GOy. $_{y}$ (69.09)

'Marina went home and Jordi to the swimming-pool.'

d. [High]/[normal/low] for specificity - obligatory agreement MARINA CAT IX3.PLU-IPSI ${ }^{36}[$ high] BUY [high] JORDI DOG

BUY[normal]. (85.08)

'Marina bought a cat non-specific, Jordi a dog $_{\text {specific.' }}$

e. [High]/[normal/low] for location - obligatory agreement MARINA SKY [high location] BIRD $_{\text {[high location] }}{ }^{37}$ LOOK $_{\text {[high 1.] JORDI }}$

DOG ŁӨӨK[low 1.]. (66.2.15)

'Marina looks at the birds in the sky and Jordi at dogs.'

\footnotetext{
${ }^{36}$ I am following Barberà's (2012) notation.

${ }^{37}$ The sign BIRD is body anchored but the eye gaze is towards a high position in the space.
} 
f. [High]/[/normal/low] iconic - optional agreement JORDI PERSON TALL+++ HELP ${ }_{[\text {high iconic] }}$ MARINA SHORT+++ HELP [low i.]. (69.16)

'Jordi helps tall people and Marina short ones.'

(Context: Jordi is very short and Mary very tall).

g. [High]/[normal/low] hierarchy - optional agreement BOSS MONEY [high hierarchy] GIVE-1 [normal] JORDI PLANT [normal]GIVE-1 1 normal]. (69.19)

'The boss gave me money and Jordi a plant.'

h. [Size/shape] (with handling CL) - optional agreement MARINA BALL BASKETBALL CL:PICK-UP[size-shape] +++ , JORDI GOLF BALL EL:PICK+++[size-shape]. (71.14)

'Marina picked up basketball balls and Jordi the golf balls.' h'. JORDI BOOK BIG THICK CL:GIVE-1 [size-shape] JOAN BOOK

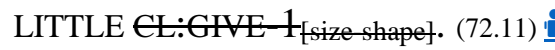

'Jordi gave me a big and thick book and Joan a little one.' h'. [Size/shape] (with instrumental CL) - optional agreement MARINA VEGETABLE CL:EAT[size-shape(fork)] JORDI APPLE EŁ:EAT[size shape(hands)]. $(68(+67) .2)$

'Marina ate vegetables (with a fork) and Jordi an apple (with his hands).'

h'", MARINA SPOON LITTLE SOUP CL:EAT[size-shape(spoon)] JORDI APPLE $\in$ L:EAT[size-shape(hands)].(72.09)

'Marina ate the soup with a little spoon and Jordi an apple.' 
The presence of mismatch in $\varphi$-features is a diagnostic that they are actual $\varphi$-features. With respect to the identity principle, though, it is necessary to give an explanation for why this can happen. Following Lasnik (1995), as we saw in 4.1.2, for similar cases of mismatch with the copula "be" in ellipsis, it seems that in gapping in LSC there is no need to have morphological identity because the syntactic identity is met before the inflection of the verbs. The identity between the conjunct is then "in form" or syntactic and not "in meaning" or semantic.

In LSC, like in spoken languages, verbs do not require morphological identity in gapping. $\varphi$-features get ignored in the resolution of gapping and their interpretation is supported also by context and world knowledge. This section gives a fine-grained description of the derivation of $\varphi$-features in LSC based on the optional or obligatory realization of a feature on the verb, supporting Bošković's (2011) approach where valued uninterpretable features can be checked or directly deleted. Finally, the underspecification of $\varphi$-features in ellipsis turns out to be a cross-linguistic and crossmodal property showing the presence of syntactic identity, and not semantic, between the conjuncts.

\subsubsection{Special cases of verbal CL and their realization in agreement and gapping}

There is a special class of CL that behave differently than the ones presented above. That is a subclass of handling and whole entity CL expressed with a restricted class of verbs: SANDWICH-EAT, PIZZA-EAT, 
ICE-CREAM-EAT and all the instances of PLAY plus any instrument. Meir (1999) describes this last group as 'patient CL' due to the argument that is, according to her, incorporated in the verb. She claims, though, that they are very rare in comparison to the rest of whole entity CL.

In LSC, this class is particularly special because of their behavior in gapping. We can see a few examples in (380). We can see that the $\mathrm{CL}$ in the first conjunct, which looks exactly like the NP the verbal CL refers to, is not interpreted in the second one. In (380a), for example, the reading is not that the referent ate a "pizzasandwich" but that she ate a pizza.

(380) a. MARC SANDWICH-EAT MARINA PIZZA. (1.01) 單

'Marc ate a sandwich and Marina a pizza.'

b. MARINA ICE-CREAM-EAT JORDI CAKE. (69.01)

'Marina ate an ice-cream and Jordi a cake.'

c. MARINA PIANO-PLAY MARC VIOLIN.

'Marina plays piano and Marc violin'.

Looking at the first conjunct in the examples in (380), it is possible to see that, differently from the other cases of CL we saw, in (380):

- the NP is not repeated outside of the VP;

- the verbal CL is not less specified than the NP, the sign is the same.

Moreover, in (381) we can see that the NP that the verbal CL is referring to can be modified even if there is no doubling. 
(381) a. MARC SANDWICH BIG SANDWICH-EAT. (70.18)

a'. MARC BIGj SANDWICH $\mathrm{j}^{-}$EAT. (70.19)

'Marc ate a big sandwich.'

Even though the test with a topicalized object and the use of resumptive pronoun cannot be applied to verbal CL in LSC to see whether they are plain or agreement verbs, it is interesting to look at the data in (382). Topicalizing the object out of the verbal CL, as in (382), or moving it via ATB movement, as in (383), it is clear that the potential agreement with the object is not necessarily realized.
a. PIZZA JORDI EAT. (46.19)

b. PIZZA JORDI PIZZA-EAT. (46.21)

'A pizza, Jordi ate.'

a. PIZZA MARINA MAKE JORDI EAT. ${ }^{38}(46.25)$

'A pizza, Marina made and Jordi ate.' a'. PIZZA MARINA MAKE THEN JORDI EAT. (46.26)

'A pizza, Marina made and then Jordi ate.'

b. PIZZA MARINA PREPARE JORDI PIZZA-EAT. (46.23)

'A pizza, Marina prepared and Jordi ate.'

The data in (381)-(383) could suggest that this small class of verbal CLs could be an instance of noun incorporation. If that was the case, though, it would be hard to explain the facts in (384), repeated from (380), where the potential incorporated noun of the first conjunct

\footnotetext{
${ }^{38}$ Jordi is not necessarily eating pizza while in (383a') he is.
} 
would be present also in the second conjunct of the gapping construction, giving the reading that the referent ate a "pizzasandwich".

\section{a. MARC SANDWICH-EAT MARINA PIZZA. (1.01)}

'Marc ate a sandwich and Marina a pizza.'

b. MARINA ICE-CREAM-EAT JORDI CAKE. (69.01)

'Marina ate an ice-cream and Jordi a cake.'

c. MARINA PIANO-PLAY MARC VIOLIN.

'Marina plays piano and Marc violin'.

A reasonable explanation could be by assuming Baker (1988); the only problem is that the absence of the NP outside the verbal CL is optional. Even though it is preferable to not have the overt NP, its absence is not obligatory. If the overt NP is produced outside the verbal CL, it would not explain its movement and how it leaves a trace behind. In the class of verbal CLs we are considering, the verbal CL takes the exact same shape of the NP outside, as we saw before for instrumental CLs. With this special class of verbal CL, the form of the verbal CL can change if the NP outside is modified.

Assuming that verbal CLs are instances of inflectional marking, and that the verbal CLs in this class correspond to the same sign of the NP they refer to, I claim that the signs SANDWICH, PIZZA, ICE-CREAM and any instruments are NPs that are nominalized verbal CLs. Moreover, the absence of doubling is explained by the need of respecting economy, avoiding using signs that are identical. Therefore, I claim that the lack of doubling is due to the use of a null 
argument licensed by the morphology of the verb. The rich morphology of the verb licenses the empty argument, as is the case in Romance languages for subjects, for instance.

Being the verbs in this class verbal CLs, they therefore carry $\varphi$-features. I argue that also this class of verbal CLs shows agreement with the referent though [size/shape]. As we saw in (379), the checking of these features on the verb is not necessary, even if very frequent. This shows that this class of nominalized verbal CLs (SANDWICH, PIZZA, ICE-CREAM and any instrument) carries a valued uninterpretable [size/shape] feature, since they can enter the derivation as goals, even though they are usually not expressed. In the latter case, they would be considered as "pro".

Since the feature is optionally realized on the verb, if it is not realized, it can be deleted from the NP without being checked. The examples we saw in (384) can then be properly glossed as in (385).

\section{a. MARC EAT[size/shape-sandwich] MARINA PIZZA. (1.01)}

'Marc ate a sandwich and Marina a pizza.'

b. MARINA EAT[size/shape-ice-cream] JORDI CAKE. (69.01)

'Marina ate an ice-cream and Jordi a cake.'

c. MARINA PLAY [size/shape-piano] MARC VIOLIN.

'Marina plays piano and Marc violin'.

The $\varphi$-features mismatch in gapping can then be explained again by the fact that in gapping in LSC there is no need to have morphological identity because the syntactic identity is met before the inflection of the verbs. 
Let us look now at a test that will show also the presence of identity in meaning in gapping in LSC, that is, adverb incorporation.

\subsubsection{Identity in meaning: adverb incorporation mismatch}

In order to establish the nature of the identity condition in LIS, other than looking at sloppy/strict readings and vehicle change, Cecchetto et al. (2015) exploit the simultaneous nature of morphology in SLs. For VP ellipsis in LIS, they get to the conclusion that identity in form is required. In LIS, as in other SLs like LSC, it is possible to incorporate a manner adverb in the verb indicating for example measure. As we can see in (386), an adverb like QUICKLY can modify the meaning of the verb in the form of a separate element (386a) or incorporated into the verb. Thanks to the simultaneous nature of SLs, the incorporation is given by a morphological change of the movement of the verb into a circular and repeated one, as in (386b), represented in Figure 20 below.

(386) a. MARIO MEAT EAT QUICKLY.

b. MARIO MEAT EAT-QUICKLY.

'Mario eats meat quickly.'

(Cecchetto et al. 2015: 227) 


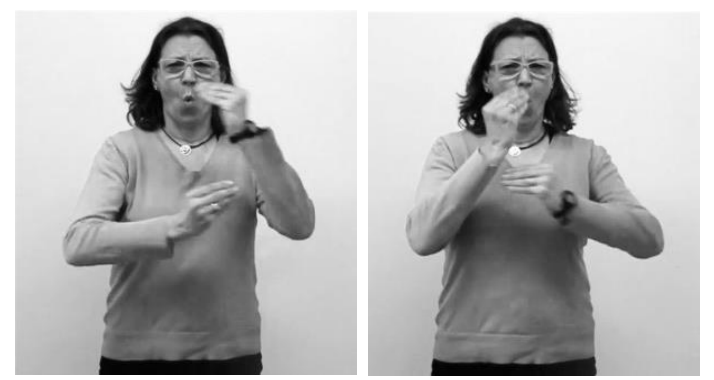

Figure 20: EAT-QUICKLY (LSC).

In LIS, when this adverb gets incorporated into the verb, though, it can also mean "a lot". In VPE in LIS, like in (387), if an adverb is incorporated in the verb in the first conjunct, it will be interpreted also in the deleted VP in the second conjunct. Therefore, if there is an adverb in the second conjunct that can potentially clash with the incorporated adverb in the first conjunct, the sentence is ungrammatical under that reading. In (387a) we can see that the only possible interpretation is the one given in (387a) because the one in (387b) would be contradictory. The only possible reading is the one where the interpretation of "eat a lot" is there in both conjuncts and then the deleted VP can be further modified by another adverb like SLOWLY in the case in (387). The reading in (387b) where "Mario eats fast slowly" in the second conjunct would lead to a contradiction. In VPE in LIS, hence, the form of the verb in the first conjunct, even if modified, needs to be the same of the one in the second conjunct.

(387) MARIO MEAT EAT-QUICKLY. GIANNI SAME SLOWLY.

a. 'Mario eats a lot of meat. Gianni does that (eat a lot of meat) slowly.' 
b. *'Mario eats meat quickly. Gianni does that (eat meat quickly) slowly.'

(Cecchetto et al. 2015: 227)

In 5.1 I argued that in LSC, differently than what Cecchetto et al. (2015) claimed for LIS, examples like the one in (387) in LSC are cases of stripping and not of VPE due to the movement of the verb from $\mathrm{V}$ to $\mathrm{T}$ with agreement verbs and the activation of $\mathrm{T}$ with plain verbs. In LSC, cases of ellipsis in which one remnant is left in the second conjunct, together with a focus additive particle, are instances of stripping. When there are more than two remnants, those are cases of gapping. Checking the data in (388) in LSC, we can see the same behavior of the adverb QUICKLY, which can also be interpreted as ALOT, as we can see in (388). The most prominent reading, though, is the first one as -LOT (388a).

(388) MARINA SPAGHETTI EAT-A-LOT/QUICKLY JORDI ALSO. (66.2.6)

a. 'Marina ate a lot of spaghetti and Jordi, too.'

b. 'Marina ate spaghetti quickly, and Jordi, too.'

Creating a more complex structure like the one in (389a), we can see that, as in VPE in LIS, in stripping in LSC the syntactic identity between the gapped site and the antecedent is kept. It is possible as well to optionally insert the additive particle ALSO without affecting the meaning. When using ALSO, an adversative construction is always preferred, like in (389b). Therefore, the adverb SLOWLY, a manner adverb generated in the vP that in stripping in (389) does not show 
any need to move out of the ellipsis site, is arguably not part of the ellipsis site itself but of a more complex structure that involves adversative coordination.

a. MARINA SPAGHETTI EAT-A-LOT JORDI EAT A LOT (ALSO) SLOWLY. (61.02)

'Marina ate a lot of spaghetti and Jordi (also) ate a lot of spaghetti slowly.'

b. MARINA SPAGHETTI EAT-A-LOT JORDI EAT-A LOT ALSO BUT SLOWLY.

'Marina ate a lot of spaghetti and Jordi also ate a lot of spaghetti but slowly.'

In LSC, then, as in VPE in LIS, it is possible to confirm the presence of syntactic identity between the elliptical site in stripping and its antecedent. When looking at gapping, though, data are different.

In (390) we can see that in gapping, if there is a remnant that is an adverb contrasting with the incorporated one, what is deleted for identity in the second conjunct is only the verb, without leading to ungrammaticality of the sentence.

(390) a. MARINA CANDY EAT-A-LOT JORDI A-LITTLE. (61.01) 르

'Marina ate a lot of candies and Jordi (ate) a little bit (of candies).'

b. MARINA CANDY EAT-A-LOT JORDI CAKE A-LITTLE.

'Marina ate a lot of candies and Jordi (ate) a little bit of cake.' 
Gapping and stripping, then, behave differently in this respect and therefore also the type of identity involved cannot be the same. For stripping we concluded that there is syntactic identity between the ellipsis site and the antecedent, but that cannot be the case for gapping, since the interpretation of the incorporated adverb in the verb depends on the presence of a contrasting adverb in the second conjunct.

Assuming the presence of identity in form in gapping, we would expect the whole head containing the adverb in the first conjunct to be interpreted in the second conjunct, too. The presence of contrast in the meaning of the two adverbs, though, makes the interpretation of the lexical meaning of the verb to be the same in the two conjuncts but not for the adverb, whose meaning contrasted. What is derived in the identity condition is then the meaning of the verb EAT and not the incorporated form with the adverb. The adverb is processed separately from the verb.

The same phenomenon, even though it is restricted to the limitation in incorporating adverbs to the verb for phonological reasons, can be found also with other verbs and adverbs. As we can see in (391a), the adverb FAST can be incorporated in the verb RUN and it can be contrasted with SLOWLY in the second conjunct. Aspectual adverbs, as in (391b), too, can be incorporated and contrasted in the second conjunct, in which the interpretation of the deleted material will include only the verb.

\section{(391) a. MARINA RUN-FAST JORDI RUN SLOWLY. (77.07) 를}

'Marina run fast and Jordi slowly.' 
b. MARINA 5-HOURS WALK+++ JORDI WALK 2 MINUTES.

'Marina walked (continually) for 5 hours and Jordi for 2 minutes.' (77.04)

Following Travis (1988) and Sportiche (1993) for spoken languages, adverb incorporation means that the adverb is a head, moves and then morphologically incorporates into the verb via head-to-head movement. Moreover, as pointed out by Cinque (1999), manner adverbs are taken to be generated in a low position, more specifically as head of vP, as pointed out by Adger \& Tsoulas (2000). Alexiadou (1997: 182), instead, proposes a structure where the adverb is a complement of VP, as in the structure in (392). Alexiadou adapts the structure by Baker (1988) for noun incorporation to adverb incorporation where the head of $\mathrm{AdvP}$ is generated as a complement of the verb and incorporation takes place. Alexiadou (1997), Rivero (1992) and Baker (1988) consider it necessary that the adverb is in a complement position, where Rivero, in turn, assumes manner adverbs to be optional complements of VP. Baker suggests that, otherwise, the trace of the adverb incorporated is not governed. I think that that will not be the case in languages that allow $\mathrm{V}$ to $\mathrm{T}$ movement. The problem, anyway, arises if there is a transitive verb. A more complex structure with double object would be necessary.

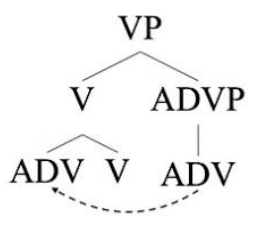

(Alexiadou 1997: 182) 
For LSC, then, I assume that manner adverbs are generated in vP, as proposed by Adger \& Tsoulas (2000), and if one happens to be incorporated, the adverb becomes part of $\mathrm{V}$ via head-to-head movement (Alexiadou 1997). After that, the verb needs to move to $\mathrm{T}$ in order to check agreement features, carrying along the incorporated adverbial morphology. The derivation of the verb, in order to compute agreement, can be represented following Costello (2016) or Pfau et al. (2018). In both cases the derivation itself does not get affected. In the case of Costello (2016) we would assume that the verb, even if plain, moves together with the adverb to $\mathrm{T}$, while for Pfau et al. (2018) the verb stays in $\mathrm{V}$ and the adverb is produced in $\mathrm{V}$ after being incorporated into the verb. In the derivation that will follow in (117), for sake of simplicity in the representation, I will adopt Costello (2016).

Going back to gapping and to the possibility of not interpreting an incorporated adverb in the second conjunct, I claim that the presence of contrast between the adverbs in the conjuncts is crucial. In stripping in LSC, as in VPE in LIS, the overt adverb in the second conjunct is not contrasting with the incorporated adverb and therefore the meaning of the verb together with the incorporated adverb is kept in identity also in the elliptical site. In gapping in LSC, instead, the overt manner adverb A-LITTLE, like in (393), is contrasting in meaning with A-LOT although the adverb A-LOT is incorporated in the verb in the first conjunct. Thanks to this contrast in meaning, in (393), repeated from (390a), it is not the incorporated form EAT-A-LOT to be interpreted in the ellipsis site but only the verb EAT. 


\section{(393) MARINA CANDY EAT-A-LOT JORDI EAT A-LITTLE. (61.01) 'Marina ate a lot of candies and Jordi (ate) a little bit (of candies).'}

The role played by the contrast in (393) between the two adverbs suggests that it is not the form that is involved in identity, but the meaning.

As for the derivation, being LSC an SOV language, the head of the phrases is found on the right for vP, VP and TP. As for the specifiers, they are always on the left. In the first conjunct, the adverb in $\mathrm{vP}$ is incorporated into the verb, which subsequently reaches $\mathrm{T}$ to check agreement features. The two remnants, instead, move to SpecTopP and SpecFocP, respectively. In the second conjunct the verb moves to $\mathrm{T}$ as well, but the adverb, which is overt, does not get incorporated. Being a remnant is therefore marked with contrastive focus and needs to move to FocP. Also Göbbel (2007), following Winkler (2005), argues that when in gapping one of the remnants is an adverb contrasting with an adverb in the first conjunct, the Contrastive topic and contrastive focus principle by Winkler (2005) is respected. We can see an example in (394), in which the subjects of the two conjuncts are assumed to be in contrastive topic, while the adverbs in contrastive focus.

(394) a. How do the older kids read to the smaller ones?

b. The ten-year old reads to the smaller kids PERFECTLY, and the nine-year old REMARKABLY WELL.

(Göbbel 2007: 293) 
In LSC, while the adverb in the first conjunct is incorporated in the verb and does not move to FocP, the whole Adverbial Phrase in the second conjunct moves up to SpecFocP. In both conjuncts, the subject goes to SpecTopP. Both TopP and FocP are in the left periphery of the sentence ${ }^{39}$. Once the whole TP in the second conjunct is evacuated from non-given material, it can get deleted. Looking at examples like the ones in (395), the incorporated adverb in the first conjunct clearly moves together with the verb because the verb with the adverb incorporated can also carry the features of the CL marking the shape of the object. Therefore, the verb together with the adverb moves to $\mathrm{T}$ to check the agreement features.

a. MARINA PIZZA CL:EAT-A-LOT JORDI A-LITTLE. (77.01)

'Marina ate a lot of pizza and Jordi a little bit.'

b. MARINA SANDWICH CL:EAT-A-LOT JORDI PIZZA A-LITTLE.

(77.02)

'Marina ate a lot of sandwiches and Jordi a little bit of pizza.'

In (397) we can see the representation of (396), repeated here again from (393). The derivation in (397) also confirms the generalization for which heads move to the right and phrases to the left.

\footnotetext{
${ }^{39}$ In Göbell (2007), the manner adverbs in 'Some ate porridge quickly and others slowly' have narrow scope. For this reason, in English, Göbell (2007) places the contrasted adverbs in a low position above VP. In LSC, differently than English, the meaning of the manner adverb in a position or another does not affect the meaning of the sentence. In LSC, then, not having this difference in meaning affected by the position of the adverbs, it is possible to have the adverbs moved higher in CP.
} 
(396)

MARINA CANDY EAT-A-LOT JORDI A-LITTLE. (61.01)

'Marina ate a lot of candies and Jordi (ate) a little bit (of candies).'

(397)

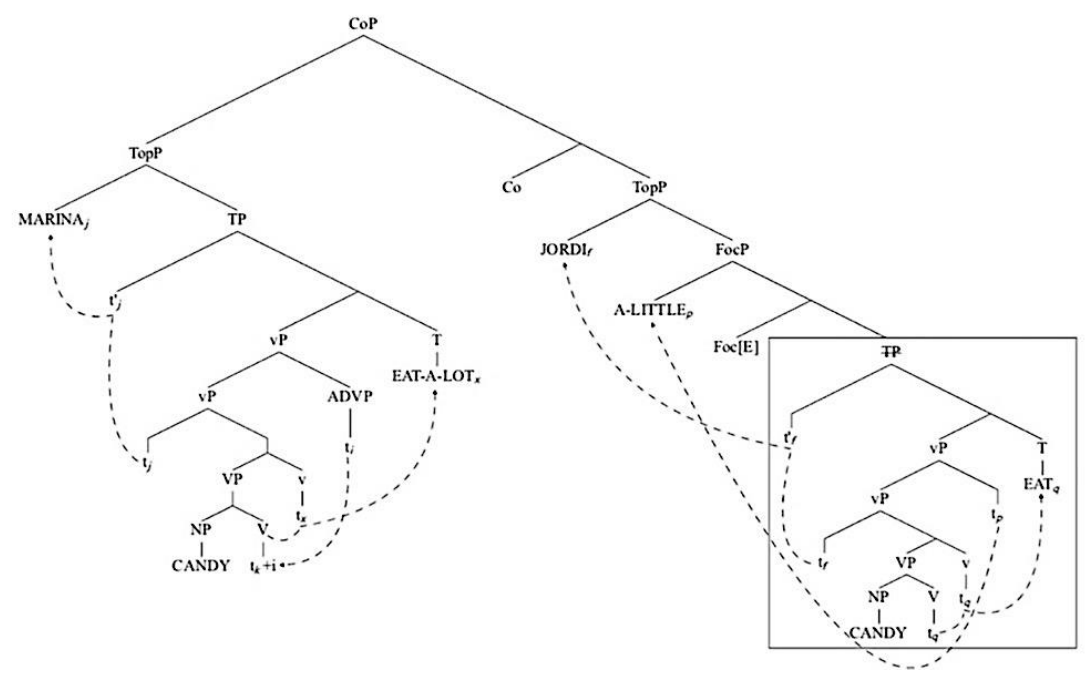

The adoption of a structure like the one in (397) can be valid when there are two remnants, but it gets more complicated when there is more than two. In (398), repeated from (390b), we can see that the subjects, the adverbs but also the direct objects of the verbs are contrasting. As far as I know, there is no literature that addresses this issue in relation to the interpretation of the remnants as contrastive topics or contrastive focus when there is more than two. Since Rizzi (1997), though, it is assumed that topics can reiterate. Therefore, I argue that when having more than one remnant, the first one bares contrastive topic, due to the fact that subjects are tendentially topicalized in SLs, the second one is marked with contrastive focus and the third one with contrastive topic. The fact that there are sets of 
alternatives is covered by the presence of contrast and for this the eGIVENness condition is respected.

(398) MARINA CANDY EAT-A-LOT JORDI CAKE A-LITTLE.

'Marina ate a lot of candies and Jordi (ate) a little bit cake.'

Considering now the example in (399), if in the second conjunct we have the adverb SLOWLY, the reading of the adverb incorporated in the verb of the first conjunct will be "eat fast", as we saw for the examples till now. In (399), though, there is also the classifier CL:ALOT that is modifying the noun PASTA. This element suggests that the subject in the first conjunct is also eating a lot, other than fast and therefore the verb in the first conjunct shows to have both readings of the adverb: EAT-FAST-A-LOT. In (399a), then, the adverb in the second conjunct is contrasting only with FAST, while A-LOT gets interpreted together with the verb and then deleted along with it. In (399b) it is possible to see also a case where adverbs that are found in different positions in the structure can be present in the gapped site. It is the case of YESTERDAY and A-LOT/FAST. While the first one is present high in the structure, the last two are generated in the vP. The adverb YESTERDAY, not being contrasted with any adverb in the second conjunct, gets deleted in the gapped site. A LOT SLOWLY. (77.11)

'Marina ate a lot of pasta fast and Jordi ate a lot of pasta slowly.' 
b. YESTERDAY MARINA PASTA CL:A-LOT EAT-FAST-A-LOT

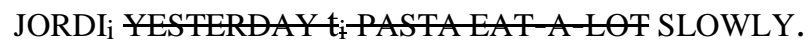

'Yesterday Marina ate a lot of pasta fast and yesterday Jordi ate a lot of pasta slowly.'

The data (399), then, show that an incorporated adverb is not interpreted in the second conjunct if an element is contrasting with it, and therefore, if there is another adverb incorporated in the verb that is not contrasted in the second conjunct, it gets interpreted in the gapped site as in the case of stripping. Both identity in meaning and form can be attested using the adverb incorporation test and the presence of contrast with the remnants is crucial in order to realize identity in meaning.

Other data that can support the importance of contrast with the remnant can also be found in other elliptical structures, like NP deletion in (400). In the first conjunct the numeral is incorporated in the noun while that is not the case in the second conjunct where only the NP, without the numeral, is deleted.

(400) MARINA PARIS 4-YEAR LIVE STAY-THERE MARC 3 YEAR.

(77.10)

'Marina lived in Paris for 4 years and Marc for 3.'

While in 5.5.1 we claimed the presence of syntactic identity or "identity in form" in gapping in LSC when looking at $\varphi$-feature mismatch, data on adverb incorporation show that identity in meaning cannot be excluded. For gapping in LSC, therefore, it is 
necessary to consider the presence of both types: in form when looking at verb agreement and in meaning, and eventually in form, in cases of adverb incorporation and only identity in meaning in licensing e-GIVENness (5.3 and 5.4).

After presenting the main questions related to the syntax and semantics of gapping in LSC, let us see next the last aspect that was first addressed in chapter 3. The syntax of gapping involves its directionality and, as already anticipated in 3.4 , this relates also to the directionality of coordination. Focusing on these two structures, it will be possible to see that the presence of prepositive coordination matches the need of having a right-branching coordination structure that justifies the presence of (forward) gapping in LSC as well.

\subsection{Directionality of coordination and of gapping: a unified account}

The definition of gapping that we have been using till now is that gapping is defined as being such only if the gap appears in the second conjunct of a coordination (Johnson 2014). This definition of gapping, though, contradicts, in part, Ross's (1970) analysis of directionality of gapping. Looking at head-final languages, Ross (1970) claims that there is no language that can only gap forward if it has SOV as underlying order (*SOV-SO). Languages like Farsi, though, as we have already seen, actually present this pattern (Carrera-Hernández 2007) and LSC does, too. This data has consequences for the directionality of the coordination structure that needs to be adopted to represent gapping. A left branching 
coordination structure (Figure 21), in fact, is not able to license the deleted material in the second conjunct. The gapped verb in the second conjunct needs to be c-commanded by the clause containing the antecedent. In Figure 21, though, the first conjunct cannot ccommand the deleted material in the second one. In the rightbranching coordination structure in Figure 22, instead, this requirement is met.

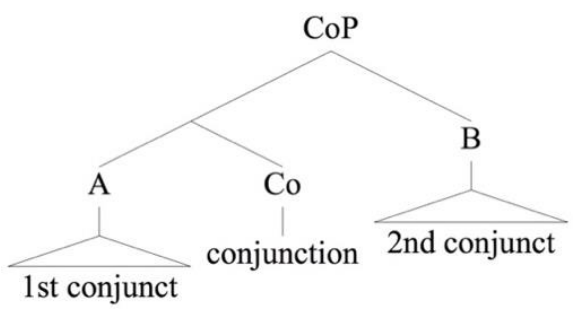

Figure 21. Left-branching coordination structure.

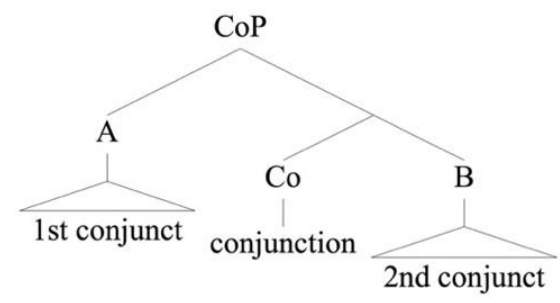

Figure 22. Right-branching coordination structure.

We will see that the type of coordination structure relates to the directionality of gapping and that SOV languages gapping forward need a right-branching coordination structure (Figure 22). This choice will be justified by the position of the coordinator with respect to the conjuncts. We will apply again the tests proposed by Haspelmath (2007) and Stilo (2004), such as intonational grouping and extraposition, which we already saw in chapter 2 for spoken languages and in 3 for LSC. 
Let us start looking at the position of the coordinator. In 2.1.3 we showed that it is possible to identify the presence of initial prepositive $(\alpha[\& \beta])$ and initial postpositive $([\alpha \&][\beta])$ coordination, together with final coordination $(\alpha[\beta \&])$, which is very rare. In this section we will only consider the first two types. Among several other tests, two effective ways to establish the position of the coordinator in relation to the conjuncts consists in looking at: i) the intonational grouping formed by the coordinator with respect to the conjuncts (Haspelmath 2007), and ii) the extraposition of the second conjunct and the position taken by the coordinator (Stilo 2004). Repeating in (401) the data that we also reviewed in chapter 2 , we can see that head-initial languages, like English (401a), and head-final ones, like Farsi (402), can show prepositive coordination. Other head-final languages like Japanese, instead, show postpositive coordination (401b). In (401) we see the intonation grouping test applied, while in (402) the extraction one is used. As in Japanese, in Farsi the coordinator cliticizes onto the element that precedes it, therefore, looking at intonational grouping, it would seem that Farsi shows postpositive coordination. Differently than Japanese, though, in Farsi extraction of the second conjunct with the coordinator is allowed. In (402) it is possible to see the contrast between (402a), which shows the basic word order, and (402b): when extraposing a conjunct, the coordinator moves along with it (Stilo 2004). Applying the extraction test to English would show the presence of prepositive coordination, as we saw in (401a) with intonational grouping. In the examples that follow, I will mark the intonational break with //. 
(401) Intonational grouping

a. $[\alpha[\& \beta]]$ in a head-initial language.

Joan // and Marvin // and their baby.

(English)

(Haspelmath 2017: 8)

b. $[\alpha \&][\beta]$ in a head-final language.

musuku-ga sotugyoo sita-si // musume-ga (Japanese)

son-NOM graduation did-and daughter- NOM

yome-ni itta.

bride-DAT went

'The son graduated and the daughter got married.'

(Zhang 2010: 13)

(402) Extraposition

$[\alpha[\& \beta]]$ in a head-final language.

a. Xoda ye dune baradar-o // ye xahar

(Farsi)

God one (CLF) brother-and one sister

dad beh-esh.

gave to-3S.OBL

b. Xoda ye (dune) bæradær dad beh-és-o ye xahær.

God one (CLF) brother gave to-3s.OBL-and one sister

'God gave him a brother and a sister.'

(Stilo 2004: 280)

As we mentioned in 2.2.1.1, the position of the coordinator can determine the orientation of the coordination structure. In Zhang's (2010) generalization, languages showing prepositive coordination will adopt a right-branching coordination structure, like for English 
(Figure 23), while languages with postpositive coordination will take a left-branching one, like for Japanese (Figure 24).

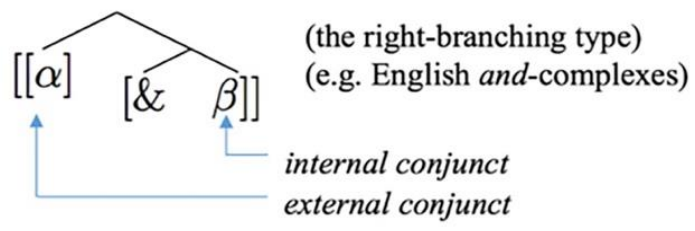

Figure 23. Right-branching structure.

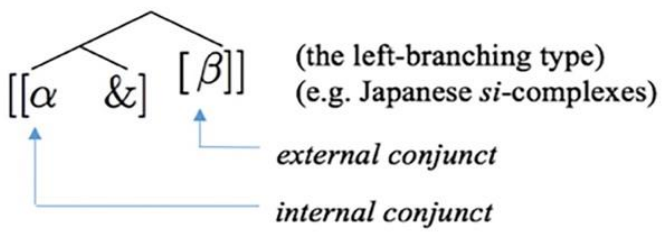

Figure 24. Left-branching structure.

Being independent of SOV or SVO order, right-branching coordination accounts also for a head-final language like Farsi (402).

Turning to LSC, as we saw in chapter 3, despite being a headfinal language, it shows prepositive coordination. We can see an example in (403) in which the coordinator ALSO is used. In (403a, a') intonational grouping is indicated, while in (403b) there is extraposition.

\begin{tabular}{rrr} 
bs contralateral & $\mathrm{hl}+$ bs ipsilarteral \\
\hline sp.con. & space ipsi.
\end{tabular}

(403) a. HOME MARINA GO ALSO TICKET CINEMA BUY PLUS--

$$
\begin{aligned}
& \frac{\text { hl ipsilateral }}{\text { s.contr. }} \\
& \text { SON SCHOOL BRING. (80.07) } \\
& \text { 'Marina went home, bought the ticket for the cinema and } \\
& \text { brought her son to school.' }
\end{aligned}
$$


hl contr. hl ipsil. hl ipsil.

a'. $\overline{\text { MARINA ICE-CREAM }} / / \overline{\text { ALSO CHOCOLATE PAY. (73.09) }}$ 'Marina paid for an ice-cream and for a chocolate.'

b. MARINA ICE-CREAM PAY, ALSO CHOCOLATE. (73.12) 'Marina paid for an ice-cream and for a chocolate.'

Therefore, as I claimed for Farsi, LSC requires a right-branching coordination structure like English (Figure 23). The rest of the LSC clause structure, though, is kept left-branching since functional heads tend to be final, while the specifier of TP has already been shown to be on the left (Benedicto et al. 2008). After the description of the properties of coordination, let us look into gapping.

As we presented earlier in this chapter, LSC, despite being an SOV language, shows only forward gapping, as in (404), while other SOV languages such as Japanese only have SO-SOV order, like in (405).

(404) a. SOV-SO

MARIA COFFEE PAY JORDI CHOCOLATE

'Marina paid for a coffee and Jordi for a chocolate.'

b. *SO-SOV

*MARINA COFFEE JoRdi CHOCOLATE PAY

(Zorzi 2018: 70) 
(405) SO-SOV

Watakusi wa sakana o, Biru wa gohan (Japanese) I (prt) fish (prt), Bill (prt) rice

o tabeta.

(prt) ate

'I ate fish and Bill rice.'

(Ross 1970: 251)

Based on the data in (404), Ross's (1970: 251) generalization, which states that "if the identical elements are on left branches, gapping operates forward; if they are on right branches, it operates backward", is not satisfied. Following Johnson's (2014) definition of gapping and the distinction between gapping and RNR (Maling (1972), Neijt (1979), Hankamer (1979), Citko (2018)), LSC shows only gapping.

An important question to raise at this point is if the rightbranching coordination structure proposed above in Figure 23 could account for gapping in LSC. In order to answer this, I argue that the difference in the directionality of the omission of the verb in coordination is also linked to the position of the coordinator itself. I claim that in languages showing initial prepositive coordination, to account for gapping, right-branching coordination is necessary, as well as in head-final languages. Head-final languages like Japanese, instead, that have initial postpositive coordination $([\alpha \&][\beta])$ and show only RNR, do not have special needs to accommodate in the structure to account for the verb missing in the first conjunct. Therefore, on the basis of the position of the coordinator and the analysis by Zhang (2010), a left-branching structure can be the proper representation for coordination and RNR in head-final languages with initial postpositive coordination. 
Other SOV languages, such as Farsi (406) and Turkish (407) show both gapping and RNR, though, which, following what has been claimed till now, would need both right and left-branching coordination.

(406) a. Gapping: SOV-SO

Rodmehr gusht xord va Anahita māhi.

(Farsi)

Rodmehr meat ate.3SG and Anahita fish

'Rodmehr ate meat and Anahita fish.'

b. RNR: SO-SOV

Rodmehr gusht va Anahita māhi xord.

Rodmehr meat and Anahita fish ate.3SG

'Rodmehr ate meat and Anahita fish.'

(Farudi 2013: 65)

(407) a. Gapping: SOV-SO

Adam kitabi okudu, çocuk da dergiyu. (Turkish) man book-ACC read-PAST child CONJ mag-ACC 'The man read the book, and the child, the magazine.'

b. RNR: SO-SOV ${ }^{40}$

Adam kitabi, çocuk da dergiyi okudu. man book- ACC child CONJ mag- ACC read-PAST 'The man read the book, and the child, the magazine.'

(Bozsahin 2000: 6)

\footnotetext{
${ }^{40}$ In Turkish, RNR also appears with the OS-OSV order. It might depend on the fact that Turkish is considered to have another underlying word order, OSV, as claimed by Bozsahin (2000).
} 
Looking first at the intonational grouping of the coordinator with each conjunct, both Farsi and Turkish display prepositive coordination. For Farsi we saw, in (402b), that when extraposing the second conjunct, the coordinator moves along. The same holds also for Turkish, in (408b), where the coordinator also forms an intonational group with the second conjunct (408a).

(408) a. Bana elma, ve armut verdiler.

(Turkish)

to.me apple and pear they.gave 'They gave me apples and pears.'

b. Bana elma verdiler ve de armu to.me apple they.gave and also apple 'They gave me apples and pears.'

Right-branching coordination, therefore, as in English and LSC, licenses gapping in Farsi and Turkish.

Finally, it is also possible to consider Polish, a language with a relative free word order that shows both gapping and RNR, as we can see in (409).

(409) a. Gapping: SVO-SO

Jan pije kawę, a Maria herbatę.

(Polish)

Jan drinks coffee and Maria tea

'Jan drinks coffee and Maria (drinks) tea.' 
b. $\mathrm{RNR}^{41}$ : SO-SOV

?Jan kawę, a Maria herbatę pije.

Jan coffee and Maria tea drinks

'Jan (drinks) coffee and Maria drinks tea.'

(Citko 2018: 1)

As for Farsi and Turkish, we would expect the need of both right and left branching coordination to account for the examples in (409). Applying the two tests we have been using till now, though, we can see that also in Polish there is prepositive coordination and therefore both structures can be derived in a right-branching coordination structure. As for intonational grouping, as already noted by Citko (2018) in (409), and as we can see in (410) below, the coordinator belongs to the second conjunct. Moreover, extraposition in coordination shows the same. In the second conjunct in (411) an SOV order is realized and the coordinator $i$ 'and' moves along with the second conjunct NP that is extraposed. In order to realize an SOV order it is necessary to have a proper context and for this reason we are considering a more complex structure in (411).

(410) Jan pije kawę// i herbatę.

Jan drinks coffee and tea

'Jan drinks coffee and tea.'

${ }^{41}$ Citko (2018) refers to it as "backward gapping". 
(411) ?Maria pije (tylko) kawę, a

(Polish)

Maria drinks (only) coffee, and

Jan kawę pije i herbatę.

Jan coffee drinks and tea

'Maria drinks only coffee and Jan drinks coffee and tea.'

In Polish, then, it is also possible to confirm that a right-branching coordination structure is at play for both gapping (SVO-SO) and RNR (SO-SOV), as also suggested by Citko (2018). While gapping is analysed as deletion at PF, RNR involves a multidominant structure (Citko 2018).

The same directionality of the structure used for RNR (SOSOV) can also be adopted for cases of RNR of an object, like in (412). It is possible to draw a parallel between the distribution of a rightnode-raised verb and a right-node-raised object in head-initial languages. Both the verb and the object are found on the extreme right of the second conjunct and they are missing in the first one, like in (412) for RNR of the object in English. Both node-raised verb and a right-node-raised object can be derived through a multidominant structure (Abels 2004; Wilder 2008; Citko 2018).

\section{(412) SV-SVO}

John bought and Mary read, a book.

This parallelism underlines, again, the importance of the position of the coordinator with respect to the conjuncts. It is not the type of ellipsis used, gapping or RNR, to determine the type of structure to 
pick for coordination, but instead it depends on whether the language has initial prepositive or postpositive coordination.

Concluding, head-final languages such as LSC, having prepositive coordination $(\alpha[\& \beta])$ and presenting forward gapping (SVO-SO), need a right-branching coordination structure. Such structure licenses the gap in the second conjunct and binding between the conjuncts. In SOV languages such as Japanese, instead, there is no such need, since it has initial postpositive coordination $([\alpha \&][\beta])$ and only RNR is used. Following Zhang (2010), these languages represent coordination with a left-branching structure. If both gapping and RNR are present in an SOV language, as in Farsi and Turkish, right-branching coordination can be used as well. They both show grouping of the coordinator with the second conjunct allowing to license gapping in a right-branching coordination structure. As for $\mathrm{RNR}$, there is no specific word order difference from SVO languages when RNRaising an object in comparison to RNRaising a verb in a head-final one. This proposal contributes to the analysis of directionality of coordination in SOV languages by underlining the importance of the position of the coordinator with respect to the conjuncts in order to decide whether a language has a right or leftbranching coordination structure. This aspect, as far as I know, has never been considered before in detail in relation to elliptical structures like gapping and RNR. 


\subsection{Summary and conclusions}

In LSC, as in spoken languages, ellipsis is a well attested phenomenon. Among the different types, which include VP ellipsis (VPE), stripping, sluicing and pseudogapping, it is also possible to find gapping. The classification of these different types of ellipsis is based on their characteristics and, even though for LSC we only looked in detail into gapping in comparison to VPE, agreement is an important aspect to consider in order to distinguish stripping and VPE in LSC. Differently than LIS (Cecchetto et al. 2015), in LSC I assume a minimalist account for agreement, following Costello (2016) and Pfau et al. (2018), in which the head of TP is always active, either through $\mathrm{V}$ to $\mathrm{T}$ movement through $\mathrm{v}$ in the case of agreement verbs, or thanks to the presence of an auxiliary in $\mathrm{T}$ or NMMs that compensate the lack of overt agreement in the space. Assuming this, then, in LSC VPE can only be found when AUX or a modal is used, otherwise it is a case of stripping. In stripping, as in VPE, only one remnant is left behind.

Focusing now on gapping in LSC, it is an attested phenomenon in which the verb is missing in the second conjunct and two or more remnants are left in contrast with their correlate antecedents, differently than stripping that only has one. The verb in gapping can only gap forward (SOV-SO) and no instance of RNR is found (*SO-SOV). Differently than other SLs like HKSL (Tang \& Lau 2012), LSC does not show any limitation in the type of verb used in gapping. All verb categories can be found and the expression of agreement in the conjuncts is compensated for by the use of space 
and NMMs. As for the syntactic properties, gapping in LSC shows different properties than English: the No embedding constraint is not respected, it is not limited to coordination, there is absence of wide scope of negation and modals but it is sensitive to islands. That LSC does not respect the No embedding constraint follows from the fact that it can appear in subordination. These similar properties with VPE makes it possible to give a unified account for both gapping and VPE, too. If other properties are not respected as in English, it does not make this structure in LSC not gapping: I argue that it is considered gapping also in LSC but it is not English-like.

The absence of wide scope of negation and modals in gapping, together with the presence of $\mathrm{V}$ to $\mathrm{T}$ movement of the verb with agreement verbs and the activation of $\mathrm{T}$ with plain ones support a large-conjunct coordination analysis for gapping in LSC. Despite the syntactic differences between gapping in LSC and English, the discourse properties are the same. Following Winkler's (2005) Contrastive topic and contrastive focus principle, it is possible to further support $\mathrm{CP}$ coordination in gapping in order to give a proper syntactic structure to discourse properties. The remnants, in fact, move to TopP and FocP in the left periphery of the sentence. The correlate antecedents move to TopP and FocP in order to respect a parallelism between the two conjuncts. This operation is also supported by the NMMs present also on the elements in the first conjunct.

As for the analysis proposed, island sensitivity shows that there is syntactic structure that is active in the ellipsis site and is able to make the sentence ungrammatical and therefore support a deletion 
account at PF. Also, the set of alternatives generated by the presence of contrast between the elements in the conjuncts make gapping in LSC compliant with Coppock's (2001) adaptation of the e-GIVENness condition proposed by Merchant (2001), according to which there is identity between the antecedent and the material deleted in the second conjunct. The syntax in the ellipsis site is considered to be the same as that of its antecedent, but the non-contrasting material, which is given, gets deleted thanks to the E-feature that indicates that the phonological value of the constituent targeted is null. For LSC, the E-feature is placed in the head of FocP to indicate that its sister, the whole TP, needs to be deleted once the arguments of the verb are evacuated to TopP and FocP. Due to the properties that gapping and VPE have in common, it is possible to propose a unified deletion account for both structures. For VPE, though, the E-feature is placed in the head of TP indicating that it is the whole vP that gets deleted.

In the classification of ellipsis in LSC, VPE is found only when an element placed in T like an auxiliary or a modal is left as remnant together with the subject of the verb in the second conjunct. If there is only one remnant left, optionally together with an additive focus particle, this would be a case of stripping. While VPE involves deletion of $\mathrm{vP}$, stripping instead is, then, a case of TP deletion. Despite the portion of the structure that gets deleted, that is TP in both stripping and gapping, it is important to distinguish these two structures, though. In gapping, in fact, it is necessary to have at least two remnants left undeleted, and those elements are in contrast with correlate antecedents in the previous conjuncts. Therefore, while for stripping there is only one element that needs to be evacuated before 
the deletion of TP, in gapping there are at least two, in line with the definition of stripping given by Hankamer and Sag (1976: 409) for which "stripping is a rule that deletes everything under identity with corresponding parts of a preceding clause except for one constituent". In order to further disentangle these two structures, it would be interesting to look at the behavior of the additive particle ALSO, which, differently than in English, can be found both in stripping and in gapping. A way to distinguish these two structures, though, can be by looking at the identity condition. It is possible to apply two modality specific tests to LSC in order to determine the presence of identity in meaning, due to semantics, or in form, due to syntax. For the syntactic identity, $\varphi$-features mismatch shows the presence of identity in form. There are several features that can be identified in LSC, among which one group is obligatorily expressed on the verb and the other optionally. Despite the optionality in agreement, when expressed in gapping, mismatch is always allowed. Following Lasnik (1995), who looks at cases of mismatch with the copula "be" in ellipsis in English, gapping in LSC does not need to have morphological identity because syntactic identity is met before the inflection of the verbs. As for identity in meaning, manner adverb incorporation mismatch shows that there is a difference between stripping and gapping in LSC. While in stripping, as in VPE in LIS (Cecchetto et al. 2015), adverb incorporation shows the presence of identity in form due to the fact that both in the ellipsis site and in the antecedent the adverb is always incorporated in the verb, this is not the case for gapping. Since in gapping there are at least two remnants, when one is an adverb contrasting with the adverb incorporated in the 
verb, then the adverb that is incorporated in the verb in the first conjunct is not present in the gapped site. Therefore, it is possible to argue that in gapping in LSC there is also identity in meaning and not just identity in form. It is true that there are cases in which it is possible to push the presence of both identity in meaning and in form, but in those examples it is necessary to be able to use an adverb that can have two meanings when it gets incorporated; also, a remnant in the second conjunct needs to contrast with one of the meanings of the adverb incorporated. In this way, the form of the adverb that is not contrasting with the remnant will be also present in the gapped site. Therefore, adverb incorporation, other than showing the presence of identity in meaning in gapping in LSC, also helps to further distinguish gapping and stripping in LSC.

Finally, the last point addresses gapping and coordination and their directionality. As first established in chapter 2, the presence of asymmetry in coordination leads to assume the presence of a right or left-branching coordination structure. As pointed out by Zhang (2010), the choice of one structure or the other depends on the position of the coordinator with respect to the conjuncts: prepositive coordination needs a right-branching coordination structure, while postpositive coordination needs a left-branching one. As for gapping, I argue that this distinction is related to the presence of (forward) gapping or RNR in a language, whether it is a head-initial or headfinal one. Considering the case of LSC, a head-final language with prepositive coordination: it is possible to support a right-branching coordination structure since it is necessary to be able to account for (forward) gapping (SOV-SO). In order to license the gapped material, 
the antecedent needs to hierarchically precede the second conjunct and this is possible only with a right-branching coordination structure. Also in head-final languages with both (forward) gapping and RNR like Farsi and Turkish, a right-branching coordination structure is justified by the presence of prepositive coordination. Assuming a multidominant analysis for RNR, a right-branching coordination structure is anyway adequate. Therefore, the position of the coordinator with respect to the conjuncts determines the directionality of coordination structure that also matches the directionality of gapping.

It is possible to conclude that gapping in LSC is not fully English-like, but it can enter the classification of elliptical structures in LSC. 


\section{FINAL REMARKS AND FUTURE DIRECTIONS}

This dissertation has shown that coordination and ellipsis, in particular gapping, are attested phenomena in Catalan Sign Language (LSC). An extensive analysis has been proposed for both structures and a considerably detailed description of the properties of each one has been provided, also in comparison with other languages. Moreover, the two syntactic analyses were connected with each other on the aspect of directionality, showing that the directionality of gapping depends on the directionality of coordination.

For coordination we focused on TP coordination and we looked at gapping in conjunction. Several questions remained open, though.

Starting with coordination, we presented data on different types of coordination, but we focused mainly on large conjunct coordination involving TPs and CPs. It would be interesting to look deeper into NP coordination, also in the eventuality of delving into NP ellipsis, and combine the two aspects. As for the different types of coordination, for conjunction we saw that tests such as checking the properties of ATB movement and scope of negation cannot be applied in LSC. We gave a first hint of an analysis suggesting that the directionality of the coordination structure is involved but it would be interesting to conduct a thorough study on these two aspects. As we saw for gapping, directionality of coordination could be related to many aspects of language cross-linguistically and crossmodally. 
With respect to the analysis we presented to distinguish conjunction and disjunction, that is at the interface between syntax and semantics involving quantifiers, it would be interesting to try to extend the study of adversative coordination along the same lines, taking into account also information structure. Moreover, adversative coordination is argued to involve ellipsis and, therefore, adding to the picture gapping in interaction with "but", following Repp (2010), would provide a more extensive analysis of this structure that in SLs is understudied.

Let us turn now to gapping. In the classification of the types of ellipsis in LSC, gapping shows different properties than in English, suggesting that it behaves like other types of ellipsis such as VPE and stripping. The distinction between the three is given by the portion of structure that is affected and the number of remnants left in the second conjunct. Both gapping and stripping, though, are argued to involve TP deletion due to the $\mathrm{V}$ to $\mathrm{T}$ movement of the verb. The main difference between the two is then based on the number of remnants, which for gapping are two or more and for stripping one. This contrast respects Hankamer \& Sag's (1976) definition of stripping and it is further justified when looking at the identity condition. In the manner adverb incorporation mismatch, in fact, in stripping it is only possible to have identity in form, while in gapping it is identity in meaning that is found. Another interesting aspect to take into consideration in the classification of ellipsis in LSC would be to look at the behavior of the additive focus particle ALSO. It appears in gapping, stripping and VPE, but the fact that it is found in gapping without affecting the meaning of the sentence is anomalous. 
Exploring this aspect further would help to give a more detailed classification of the types of ellipsis and their interaction with focus particles such as ALSO.

As for the environment in which gapping can be found in LSC, we focused on conjunction, but no attention was given to disjunctive or adversative coordination. As well, it would be important to further focus on gapping in subordination.

Finally, in the analysis that puts together the directionality of coordination and gapping, incorporating more languages to the generalization would help to make a stronger claim. The addition of head-final languages that show postpositive coordination like Japanese without being enclitic or allowing extraposition would make the generalization among different languages clearer.

I am sure that there are still plenty of unsolved questions in relation to coordination and ellipsis in LSC and sign languages in general, but the ones presented here naturally come out of this dissertation and delineate what can be done as a natural follow-up in the future. 
Coordination and gapping in Catalan Sign Language (LSC) 


\section{REFERENCES}

Aarons, Debra. 1994. Aspects of the Syntax of American Sign Language. Boston, MA: Boston University dissertation.

Abels, Klaus. 2004. Right Node Raising: Ellipsis or across the board movement. In Keir Moulton and Matthew Wolf (eds.), Proceedings of NELS 34, Amherst: GLSA, pp. 45-59.

Adger, David \& George Tsoulas. 2000. Aspect and lower VP adverbials. In Alexiadou, Artemis \& P. Svenonius (eds.) Adverbs and Adjunction, pp. 1-18. Linguistics in Potsdam 6. Potsdam.

Aelbrecht, Lobke. 2009. You have the right to remain silent: The syntactic licensing of ellipsis. Brussels: Catholic University of Brussels dissertation.

Aelbrecht, Lobke. 2010. The syntactic licensing of ellipsis. Amsterdam: John Benjamins.

Ai, Ruixi Ressy. 2014. Topic-comment structure, focus movement, and gapping formation. Linguistic Inquiry 45(1). 125-145.

Ala-Sippola, Sanna. 2012. Agentin ilmaiseminen suomalaisella viittomakielella $\cdot$ tuotetussa asiatekstissa [Expressing agent in a formal FinSL narrative]. Department of Modern Languages, University of Helsinki MA thesis.

Alexiadou, Artemis. 1997. Adverb placement: A case study in Antisymmetric syntax. Amsterdam: Benjamins.

Alonso-Ovalle, Luis. 2006. Disjunction in alternative semantics. Amherst, MA: University of Massachusetts dissertation. 
Bahan, Benjamin. 1996. Non-manual realization of agreement in ASL. Boston, MA: Boston University dissertation.

Bahan, Benjamin, Judy Kegl, Robert G. Lee, Dawn MacLaughlin \& Carol Neidle. 2000. The licensing of null arguments in American Sign Language. Linguistic Inquiry 31(1), 1-27.

Baker, Mark. 1988. Incorporation. A Theory of Grammatical Function Changing. Chicago: Chicago University Press.

Barberà, G. 2014. Use and functions of spatial planes in Catalan Sign Language discourse. Sign Language Studies 14.

2015. The Meaning of Space in Sign Language. Reference, Specificity and Structure in Catalan Sign Language Discourse. Berlin/Boston: De Gruyter Mouton and Ishara Press.

2016. Indefiniteness and specificity marking in Catalan Sign Language (LSC). Sign Language \& Linguistics 19 (1): 1-36.

Barberà, Gemma \& Mosella, Marta. 2014. Les llengües de signes: Gramàtica i modalitat gestovisual. Un estudi de cas de la concordança verbal en la llengua de signes catalana (LSC). Llengua i literatura 24: 111-151.

Barberà, Gemma \& Cabredo Hofherr, Patricia. 2017. Two indefinite pronouns in Catalan Sign Language (LSC). Talk presented at Sinn und Bedeutung 21, September 4-6, University of Edinburgh. 
Beavers, John, \& Ivan A. Sag. 2004. Coordinate ellipsis and apparent non-constituent coordination. In Müller, Stefan (ed.), Proceedings of the HPSG04 conference, Katholieke Universiteit Leuven, 48-69. Stanford, Calif.: CSLI Publications.

Bellert, Irene. 1972. On certain syntactical properties of the English connectives and and but. In Senta Plötz (ed.), Transformationelle Analyse: Die Transformationstheorie von Zellig Harris und ihre Entwicklung, 327-356. Frankfurt am Main: Athenäum Verlag.

Benedicto Elena, Brentari Diane. 2004. Where did all the arguments go?: Argument-changing Properties of Classifiers in ASL. Natural Language and Linguistic Theory 22(4):1-68.

Blakemore, Diane, \& Robyn Carston. 1999. The Pragmatics of andconjunctions: The Non-narrative Cases. UCL Working Papers in Linguistics 11:1-20.

Bolinger, Dwight. 1967. The imperative in English. In To honor Roman Jakobson: Essays on the occasion of his seventieth birthday, volume 1 of Janua Linguarum, 335-362. The Hague and Paris: Mouton.

Borsley, Robert D. 2005. Against ConjP. Lingua 115: 461-482.

Bošković, Željko. 2011. On Unvalued Uninterpretable Features. Proceedings for NELS 39.

Bosque, Ignacio. 1980. Sobre la negacion. Madrid: Catedra.

Bozsahin, Cem. 2000. Gapping and word order in Turkish. Studies in Turkish linguistics:proceedings of the Tenth International 
Conference in Turkish Linguistics. August 16-18, 2000, Boğaziçi University, İstanbul.

Bril, Isabelle. 2002. Le Nêlêmwa. Analyse syntaxique et sémantique. Paris: Peeters.

Büring, Daniel. 2002. Question-Answer congruence - unstructured comments on Krifka (2001). Berlin: ZAS.

Camacho, José Antonio. 1997. The Syntax of NP Coordination. Los Angeles, CA: University of Southern California dissertation.

Cañas, Sara. 2018. Nonmanual marking of polar interrogatives in Catalan Sign Language (LSC): Approaching the puzzle through a feature-based theory of biases. Talk presentated at IGG44, March 1-3, Rome (Italy).

Carminati, Maria Nella. 2002. The Processing of Italian Subject Pronouns, Ph.D. dissertation, University of Massachusetts at Amherst, Amherst (MA), GLSA publications.

Cecchetto, Carlo, Alessandra Checchetto, Carlo Geraci, Mirko Santoro, \& Sandro Zucchi (2015). The syntax of predicate ellipsis in Italian Sign Language (LIS). Lingua 166: 214-235.

Chierchia, Gennaro. 1993. Questions with Quantifiers. Natural Language Semantics 1, 181-234.

Chomsky, Noam. 1957. Syntactic Structures. The Hague: Mouton 1981. Lectures on Government and Binding. Foris:Dordrecht. 1995. The Minimalist Program. Cambridge: MIT Press. 
Chung, Sandra. 2006. Sluicing and the lexicon: The point of no return. In Proceedings of the annual meeting of the Berkeley Linguistics Society 31. Cover, Rebecca T. \& Yuni Kim (eds.), 73-91. Berkeley, Calif.: Berkeley Linguistics Society.

Chung, Sandra, William A. Ladusaw, \& James McCloskey. 1995. Sluicing and Logical Form. Natural Language Semantics 3:239-282

Cinque, Guglielmo. 1999. Adverbs and Functional Heads. A Crosslinguistic Perspective. New York: Oxford University Press.

Citko, Barbara. 2005. On the Nature of Merge: External Merge, Internal Merge, and Parallel Merge. Linguistic Inquiry $36: 475-496$.

2007. Disambiguation Strategies in Across-theBoard-Wh-Questions. In proceedings of BLS 33, No 1, Berkeley Linguistics Society and the Linguistic Society of America.

2018. On the Relationship between Forward and Backward Gapping. Syntax 21:1, 1-36.

Clayre, B. \& L. Kubit. 1974. An outline of Kayan grammar. In Rousseau, J. (ed.), The peoples of Central Borneo (Sarawak Museum Journal) special issue Vol. 22, no. 43 new series, 4391. Kuching: Sarawak Museum.

Collins, Chris. 1988. Part 2. Alternative Analyses of Conjunction. Manuscript, MIT.

Coppock, Elizabeth. 2001. Gapping: in defense of deletion. In Chicago Linguistics Society, ed. Mary Andronis, Christoper 
Ball, Heidi Elston, and Sylvain Neuvel, volume 37, 133-148. University of Chicago.

Corver, Norbert \& Marjo van Koppen. 2010. Ellipsis in Dutch possessive noun phrases: A micro-comparative approach. Journal of Comparative Germanic Linguistics 13:99-140.

Corver, Norbert, \& Marjo van Koppen. 2011. NP-ellipsis with adjectival remnants: a micro-comparative perspective. Natural Language and Linguistic Theory 29:371-421.

Costello, B. D. N. 2016. Language and modality: Effects of the use of space in the agreement system of lengua de signos española (Spanish Sign Language). Utrecht: LOT.

van Craenenbroeck, Jeroen. 2008. What does silence look like? On the unpronounced syntax of sluicing. Talk presented at the University of Chicago.

2010. The syntax of ellipsis: Evidence from Dutch dialects. New York, NY: Oxford University Press.

van Craenenbroeck, Jeroen, \& Anikó Lipták. 2006. The crosslinguistic syntax of sluicing: Evidence from Hungarian relatives. Syntax 9:248-274.

Crasborn, Onno \& Els van der Kooij. 2013. The phonology of focus in Sign Language of the Netherlands. Journal of Linguistics 49(3). 515-565.

Crysmann, Berthold. 2006. Coordination. In Brown, K. (ed.), Encyclopedia of Language and Linguistics, Elsevier, Oxford.

Culicover, Peter W. \& Ray Jackendoff. 2005. Simpler Syntax. Oxford: Oxford University Press. 
Dalrymple, Mary. 1991. Against reconstruction in ellipsis. Unpublished ms., Xerox-PARC and CSLI.

Davidson Zaremba, Kathryn. 2011. The Nature of the Semantic Scale: Evidence from Sign Language Research. San Diego, CA: University of California dissertation.

2013. 'And' or 'or': General use coordination in ASL. Semantics \& Pragmatics Volume 6, Article 4: 1-44, 2013 http://dx.doi.org/10.3765/sp.6.4

Du Feu, Veronica. 1996. Rapanui. Descriptive Grammars. London; New York: Routledge.

Farudi, Annahita. 2013. Gapping in Farsi: A cross-linguistic investigation. Amherst, MA: University of Massachusetts at Amherst dissertation.

Féry, Caroline \& Vieri Samek-Lodovici. 2006. Focus projection and prosodic prominence in nested foci. Language 82(1):131150.

Fiengo, Robert, \& Robert May. 1994. Indices and identity. Cambridge, Massachusetts: MIT Press.

Fischer, Susan D. 1975. Influences on word order change in American Sign Language. In Li, Charles (ed.) Word order and word order change. Austin: University of Texas Press, $1-25$.

Fortin, Catherine. 2007. Indonesian sluicing and verb phrase ellipsis: Description and explanation in a minimalist framework. Ann Arbor, MI: University of Michigan dissertation. 
Fox, Danny. 2000. Economy and semantic interpretation. Cambridge, Massachusetts: MIT Press.

Frazier, Michael, \& Yoshida, Masaya. 2012. Remarks on Gapping in ASL. Snippets 26.

Frazier, Lyn; Clifton, Charles Jr \& Carlson, Katy. 2007. Focus and VP ellipsis. Lang Speech. 2007; 50(Pt 1): 1-21.

Gengel, Kirsten. 2006. Contrastivity and ellipsis. In Mackie, L. and A. McNay (eds.), Online Proceedings of LingO: The First University of Oxford Postgraduate Conference in Linguistics.

Ginzburg, Jonathan, \& Ivan A. Sag. 2000. Interrogative investigations. Stanford, Calif.: CSLI Publications.

Gleitman, Lila R. (1965). Coordinating conjunctions in English. Language, 41, 260-293.

Glück, Susanne \& Pfau, Roland. 1998. On classifying classification as a class of inflection in German Sign Language. Proceedings for ConSOLE 6

Gökgöz, Kadir \& Engin Arik. 2011. Distributional and syntactic characteristics of nonmanual markers in Turkish Sign Language (Turk İşaret Dili, TID). MIT Working Papers in Linguistics 62. Proceedings of the 7th Workshop on Altaic Formal Linguistics. 63-78.

Göksel, Asli \& Kelepir, Meltem. (2016). Observations on clausal complementation in Turkish Sign Language. In Herrmann, Annika \& R. Pfau \& M. Steinbach (eds.), A Matter of Complexity: Subordination in Sign Languages. Berlin: De Gruyter Mouton. (pp. 65-94) 
Göbbel, Edward. 2004. Focus and marked positions for VP adverbs. Ms., University of Tübingen.

Goodall, Grant. 1987. Parallel Structures in Syntax: Coordination, Causatives and Restructuring. Cambridge: Cambridge University Press.

Grosu, Alexander. 1972. The strategic content of island constraints. The Ohio State University Working Papers in Linguistics 13 (special issue; revised doctoral thesis).

1973. On the nonunitary nature of the Coordinate Structure Constraint. Linguistic Inquiry No. 4.

Ha, Seungwan. 2008. Contrastive focus: Licensor for Right Node Raising. In Elfner, Emily and Martin Walkow (eds.) Proceedings of NELS 37, volume 247-260. Amherst, Mass.: GLSA, University of Massachusetts.

Hamblin, Charles L. 1973. Questions in Montague English. Foundations of Language 10(1). 41-53.

Hankamer, Jorge. 1979. Deletion in coordinate structures. New York: Garland Publishing, Inc.

Hankamer, Jorge, \& Ivan Sag. 1976. Deep and surface anaphora. Linguistic Inquiry 7.3:391-426.

Hardt, Daniel. 1993. Verb phrase ellipsis: Form, meaning and processing. University of Pennsylvania dissertation.

1999. Dynamic interpretation of verb phrase ellipsis. Linguistics and Philosophy 22:185-219. 
Hartmann, Katharina. 2000. Right node raising and gapping: Interface conditions on prosodic deletion. John Benjamins.

2015. Coordination in Syntax - Theory and Analysis An International Handbook. Kiss, Tibor \& Alexiadour, Artemis (eds.), Mouton de Gruyter, Berlin.

Haspelmath, Martin. 1993. A grammar of Lezgian. Mouton de Gruyter, Berlin.

2004. Coordinating constructions (Typological Studies in Language, 58. Benjamins.

2007. Coordination. In Shopen, Timothy (ed.) Language typology and syntactic description, vol. II: Complex constructions. 2nd edn. Cambridge: Cambridge University Press, 1-51.

Hernández, Ana Carrera. 2007. Gapping as a syntactic dependency. Lingua 117. 2016-2133.

Herrmann, Annika. 2010 The Interaction of Eye Blinks and Other Prosodic Cues in German Sign Language. In: Sign Language \& Linguistics 13(1), 339.

Hoeksema, Jacob \& Donna Napoli. 1993. Paratactic and Subordinative So. Journal of Linguistics 29:2, 291-314.

Hudson, Richard A. 1976. Conjunction reduction, gapping and rightnode raising. Language 52:535-562.

Ince, Atakan. 2009. Dimensions of ellipsis: Investigations in Turkish. Ph.D. Dissertation, University of Maryland, College Park. 
Jackendoff, Ray S. 1971. Gapping and related rules. Linguistic Inquiry 2:21-35.

1977. X'Syntax. Cambridge, Mass: MIT Press.

Jacobson, Pauline. 2003. Binding without pronouns (and pronouns without binding). In G.-J. M. Kruijff \& R. T. Oehrle (eds.), Resource-Sensitivity, Binding and Anaphora, pages 57-96. Dordrecht: Kluwer.

Jantunen, Tommi. 2013. Ellipsis in Finnish Sign Language. Nordic Journal of Linguistics 36(3), 303-332.

2016. Clausal coordination in Finnish Sign Language. Studies in Language 40:1 (2016), 2014-234.

Jayaseelan, K. A. 1990. Incomplete VP deletion and Gapping. Linguistic Analysis 20:64-81.

Johannessen, Janne Bondi. 1998. Coordination. Oxford: Oxford University Press.

Johnson, Kyle. 2004. In search of the English middle field. Ms, University of Massachusetts, Amherst.

2009. Gapping is not (VP) Ellipsis. Linguistic Inquiry 40.2, 289-328.

2014. Gapping. Manuscript, University of Massachusetts at Amherst.

Johnston, Trevor \& Schembri, Adam. 2007 Australian Sign Language: An Introduction to Sign Language Linguistics. Cambridge: Cambridge University Press. 
Jung, Wonsuk. 2016. The non-unity of gapping. Euskal Herriko Unibertsitatea-University of the Basque Country dissertation.

Kayne, Richard S. 1994. The Antisymmetry of Syntax. Cambridge: MIT Press.

Kehler, Andrew. 2002. Coherence in discourse. Stanford, Calif.: CSLI Publications.

Kimmelman, Vadim. 2017. Linearization of weak hand holds in Russian Sign Language. Linguistics in Amsterdam 10,1 (2017), 28-59.

Kooij, Els van der, Onno Crasborn \& Wim Emmerik. 2006. Explaining prosodic body leans in Sign Language of the Netherlands: pragmatics required. Journal of Pragmatics 38. 1598-1614.

Kratzer, Angelika. 1996. Severing the external argument from its verb. In Rooryck, Johan \& Laurie Zaring (eds.), Phrase structure and the lexicon, 109-137. Dordrecht, The Netherlands: Kluwer Academic Publishers.

Krifka, Manfred. 2008. Basic notions of information structure. Acta Linguistica Hungarica 55(3):243-276.

Kuno, Susumu. 1976. Gapping: a functional analysis. Linguistic Inquiry 7:300-318.

1982. The Focus of the Question and the Focus of the Answer. In Papers from the Parasession on Non declaratives, Chicago Linguistics Society, 134-157. 
Lakoff, George. 1971. The role of deduction in grammar. In Filmore, C. J. \& D. T. (eds.) Studies in Linguistic Semantics. New York: Holt, Rinehart and Winston.

1986. Frame Semantic Control of the Coordinate Structure Constraint. In Anne Farley, M. \& Peter T. Farley \& Karl-Erik McCullough (eds.), Papers from the Parasession on Pragmatics and Grammatical Theory.

Lakoff, George \& Peters, Stanley. 1966. Phrasal Conjunction and Symmetric Predicates. In Report NSF 17, Computation Laboratory, Harvard University VI-1 to VI-49.

Larson, Richard K. 1985. On the syntax of disjunction scope. Natural Language and Linguistic Theory 3, 217-264.

Legeland, Iris, Katharina Hartmann \& Roland Pfau. 2018. Word order asymmetries in NGT coordination: The impact of Information Structure. Talk presentated at FEAST 2018, June 18-10, Venice (Italy).

Lausberg, Hedda, \& Sloetjes, Han. 2009. Coding gestural behavior with the NEUROGES-ELAN system. Behavior Research Methods, Instruments, \& Computers, 41, 841-849.

Levin, Nancy S. 1986. Main-verb ellipsis in spoken English. New York: Garland Publishing.

Levin, Nancy S., \& Ellen F. Prince. 1986. Gapping and causal implicature. Papers in Linguistics 19:351-364.

Liddell, Scott. 1980. American Sign Language Syntax. The Hague: Mouton. 
2003 Grammar, Gesture, and Meaning in American Sign Language. Cambridge: Cambridge. University Press.

Lillo-Martin, Diane. 1991. Universal Grammar and American Sign Language: Setting the Null Argument Parameters. Dordrecht: Kluwer.

1995. The point of view predicate in American Sign Language. In Emmorey, Karen \& Judy Reilly (eds.), Language, gesture, and space, 155-170. Hillsdale, NJ: Lawrence Erlbaum Associates.

Lin, Vivian. 2000. Determiner sharing. In Billerey and Lillehaugen (eds.), WCCFL 19 Proceedings, volume 19, 274-287.

2001. A way to undo A-movement. In Megerdoomian, K. \& L.A. Barel (eds.) WCCFL 20 Proceedings, 358-371.

2002. Coordination and sharing at the interfaces. Cambridge, MA: MIT dissertation.

Lobeck, Anne. 1995. Ellipsis: Functional heads, licensing and identification. New York: Oxford University Press.

López, Luis \& Susanne Winkler. 2002. Gapping in phases. In Schwabe, Kerstin and Susanne Winkler (eds.), The Interfaces: Deriving and Interpreting (Omitted) Structures, John Benjamins: Amsterdam.

Maling, Joan M. 1972. On "gapping and the order of constituents". Linguistic Inquiry 3:101-108.

Mathur, Gaurav. 2000. Verb agreement as alignment in signed languages. Cambridge, MA: MIT dissertation. 
Mauri, Caterina. 2008. Coordination relations in the Languages of Europe and Beyond. EALT series, Berlin, New York: Mouton de Gruyter.

McCawley, J.D. 1993. Gapping with shared operators. In Proceedings of the 19th Annual Meeting of the BLS: General Session and Parasession on Semantic Typology and Semantic Universals, 245-253.

Meir, Irit. 1999. A perfect marker in Israeli Sign Language. Sign Language \& Linguistics 2 (1), 43-62.

Merchant, Jason. 2001. The syntax of silence: Sluicing, islands, and the theory of ellipsis. Oxford University Press: Oxford.

2004. Fragments and ellipsis. Linguistics and Philosophy 27, 661-738.

2006. Rethinking the identity conditions in ellipsis. Ealing 2006, Paris.

2012. Ellipsis. Submitted to Syntax: An international handbook of contemporary syntactic research, Tibor Kiss \& Artemis Alexiadou (eds.), Berlin: Walter de Gruyter.

2013a. Diagnosing ellipsis. In Lisa Lai-Shen Cheng \& Nobert Corver (eds.), Diagnosing syntax, 537-542. Oxford University Press: Oxford.

2013b. Voice and Ellipsis, Linguistic Inquiry 44(1): 77-108.

Mithun, Marianne. 1988. The Grammaticalization of Coordination. In Haiman, John \& Thompson, Sandra A. (eds.), Clause 
Combining in Grammar and Discourse, 331-359. Amsterdam: John Benjamins.

Moltmann, Frederike. 1992. On the Interpretation of ThreeDimensional Syntactic Trees. Proceedings of SALT II.

Mosella, Marta. 2012. Les construccions relatives en llengua de signes catalana ( $L S C)$. Barcelona: Universitat de Barcelona dissertation.

Muadz, Husni. 1991. A Planar Theory of Coordination. Tucson, AZ: University of Arizona dissertation.

Munn, Alan Boag. 1987. Coordinate Structure and X-bar theory, McGill Working Papers in Linguistics 4(1), 121-140.

1992. A null operator analysis of ATB gaps. The Linguistic Review 9,1-26.

1993. Topics in the syntax and semantics of coordinate structures. University of Maryland dissertation.

1999. On the identity requirement of ATB extraction. Natural Language Semantics 7, 421-425.

Na, Younghee \& Huck, Geoffrey J. 1992. On extracting from asymmetrical structures. In Brentari, D. \& G. Larson, \& L. Macleod (eds.), The Joy of Grammar: A Festschrift in Honor of James D. Mccawley, pp. 251-274. Amsterdam/Philadelphia: John Ben- jamins.

Navarrete González, Alexandra. 2016. Focus constructions in Catalan Sign Language (LSC). Barcelona: Universitat Pompeu Fabra MA thesis. 
2018. The expression of focus and its relation to contrast in Catalan Sign Language (LSC). Talk presentated at II Encontro sobre Morfossintaxe da LGP $e$ outras Linguas de SinaisII - Meeting on Morphosyntax of LGP and other Sign Languages. FLUP e ESE/PP.

Neijt, Anneke. 1979. Gapping: a contribution to sentence grammar. Dordrecht, The Netherlands: Foris Publications.

Nonato, Rafael. 2014. Clause chaining, switch reference and coordination. Cambridge, MA: MIT dissertation.

Nunes, Jairo. 1995. The Copy Theory of Movement and Linearization of Chains in the Minimalist Program. University of Maryland at College Park dissertation.

Oehrle, Richard T. 1987. Boolean properties in the analysis of gapping. In Geoffrey J. Huck (ed.), Discontinuous constituency, Syntax and semantics, volume 20, 203-238. Academic Press, Inc. Harcourt Brace Jovanovich.

Oversteegen, Leonoor E. 1997. On the pragmatic nature of causal and contrastive connectives. Discourse Processes 24. 1, 51-86.

Padden, Carol A. 1983/1988. Interaction of morphology and syntax in American Sign Language. New York: Garland.

Partee, Barbara H. \& Mats Rooth. 1983. Generalized conjunction and type ambiguity. In Rainer, Bäuerle \& Christoph Schwarze \& Arnim von Stechow (eds.), Meaning, Use and Interpretation of Language, 361-383. Berlin: Walter de Gruyter.

Pearson, Matt. 2006. What's no! Focusand clause linking in Malagasy. Paper presented at the Comparative. Austronesian Syntax Workshop, 6-8 Oct. 2006, UCSD. 
Peterson, Peter G. 2004. Coordination: consequences of lexicalfunctional account. Natural Language and Linguistic Theory 22, 643-679.

Pfau, Roland \& Quer, Josep. 2007. On the syntax of negation and modals in German Sign Language (DGS) and Catalan Sign Language (LSC). In Perniss, Pamela \& Pfau, Roland \& Steinbach, Markus (eds.), Visible Variation. Comparative Studies on Sign Language Structure. Berlin: Mouton de Gruyter. 129-161.

2010. Nonmanuals: Their Grammatical and Prosodic Roles. In Brentari, Diane (ed.), Sign Languages: A Cambridge Language Survey. Cambridge: Cambridge University Press, 381402.

Pfau, Roland \& Markus Steinbach. 2008. Agreement auxiliaries and transitivity in sign languages. Talk presented at Workshop on transitivity, November 14-15, Universität zu Köln, Cologne, Germany.

Pfau, Roland, Martin Salzmann \& Markus Steinbach. 2018. Sign Language Agreement. Common Ingredients but Unusual Recipe. Manuscript University of Amsterdam, University of Leipzig and University of Göttingen. Submitted.

Pesetsky, David. 2000. Paths and categories. Cambridge, MA: MIT dissertation.

Pesetsky, David and Torrego Esther. 2007. The syntax of valuation and the interpretability of features. In S. Karime, V. Samiian \& W. Wilkins. (eds.), Phrasal and Clausal Architecture: Syntactic derivation and interpretation, 262-294. Amsterdam/ Philadelphia: Benjamins. 
Potter, David, Michael Frazier, Masaya Yoshida. 2015. A Two Source Hypothesis for Gapping. In Natural Language \& Linguistic Theory.

Postal, Paul Martin. 1998. Three Investigations of Extraction. The MIT Press.

1974. On raising. Cambridge, Massachusetts: MIT Press.

Potsdam, Eric. 1997. English verbal morphology and VP ellipsis. In Meeting of the North Eastern Linguistic Society. GLSA.

Progovac, Ljiljana. 1998. Structure for Coordination. Part I and II.' Glot International 3.7: 3-6 and 3.8: 3-9.

Quadros, Ronice Müller de. 1999. Phrase structure of Brazilian Sign Language. Pontificia Universidade Católica do Rio Grande do Sul dissertation.

Quadros, Ronice Müller de \& Josep Quer. 2008. Back to back(wards) and moving on: on agreement, auxiliaries and verb classes in sign languages. In de Quadros, Ronice M. (ed.), Sign Languages: spinning and unraveling the past, present and future. TISLR9, forty five papers and three posters from the 9th. Theoretical Issues in Sign Language Research Conference, Florianopolis, Brazil, December 2006, 530-551. Petrópolis/RJ, Brazil: Editora Arara Azul.

Quer, Josep. 2002. Negative operators in Catalan Sign Language.

Ms., ICREA \& Universitat de Barcelona.

2005. Context shift and indexical variables in sign languages. In SALT XV. Ithaka, NY: CLC Publications. 
2011. When agreeing to disagree is not enough: Further arguments for the linguistic status of sign language agreement. Theoretical Linguistics 37-3/4, 189-196. Walter de Gruyter.

Quer, Josep, Laura Mazzoni \& Galini Sapountzaki. 2010. Sign Languages in Mediterranean Europe. In D. Brentari, Cambridge Language Surveys: Sign Languages, 95-112. Cambridge: Cambridge University Press.

Quer, Josep \& Joana Rosselló. 2013. On sloppy readings, ellipsis and pronouns: Missing arguments in Catalan Sign Language (LSC) and other argument-drop languages, V. CamachoTaboada, A.L. Jiménez-Fernández, MartínGonzález \& Mariano Reyes-Tejedor (eds.) Information Structure and Agreement, pp. 337-370.

Quirk, Randolph; Greenbaum, Sidney; Leech, Geoffrey; Svartvik, Jan. 1985. A Comprehensive Grammar of the English Language. Longman, London.

Rathmann, Christian \& Gaurav Mathur. 2008. Verb agreement as a linguistic innovation in signed languages. In Quer, Josep (ed.), Signs of the time. Selected papers from TISLR 8. Hamburg: Signum, 191-216.

Repp, Sophie. 2016. Contrast: Dissecting an elusive informationstructural notion and its role in grammar. In Féry, Caroline \& Shinichiro Ishihara (eds.), OUP Handbook of Information Structure.Oxford: Oxford University Press, 270-289.

2009. Negation in gapping. Number 22 in Oxford studies in theoretical linguistics. Oxford University Press. 
2010. Defining 'contrast' as an information-structural notion in grammar. Lingua 120(6), 1333-1345.

Riemsdijk, H. van. 2006. Grafts follow from Merge. In Frascarelli, M. (ed.) Phases of Interpretation, 17-44.

Rivero, María-Luisa. 1990. The location of nonactive voice in Albanian and Modern Greek. Linguistic Inquiry 21:135-146. 1992. Adverb Incorporation and the Syntax of Adverbs in Modern Greek. Linguistics and Philosophy 15: 289-331.

Rizzi, Luigi. 1997. The Fine Structure of the Left Periphery. In Liliane Haegeman (ed.), Elements of Grammar, pp. 281-337, Kluwer Academic Publishers, Dordrecht.

Rooth, Mats. 1992. A theory of focus interpretation. Natural Language Semantics 1:117-121.

Rosen, Sara T. 1989. Two types of noun incorporation: A lexical analysis. Language 65, pp. 294- 317.

Ross, John R. 1967. Constraints on variables in syntax. Cambridge, MA: MIT dissertation.

1970. Gapping and the order of constituents. In Manfred Bierwisch and Karl E. Heidolph (eds.), Progress in linguistics,. The Hague: Mouton, pp. 249-259.

Ross, Daniel. 2013. Verbal Pseudocoordination in English: A syntactic analysis with reference to diachronic, dialectal and cross-linguistic variation. Unpublished Qualifying Exam paper, University of Illinois at Urbana-Champaign. 
Ruys, E.G. 1992. The scope of indefinites. Utrecht University dissertation.

Sag, Ivan. 1976. Deletion and logical form. Cambridge, MA: MIT dissertation.

1980. Deletion and logical form. New York: Garland Publishing.

Sag, Ivan, Gerald Gazdar, Thomas Wasow, and Steven Weisler. 1985. Coordination and how to distinguish categories in Natural Language and Linguistic Theory 3: 117-171.

Schlangen, David. 2003. A coherence-based approach to the interpretation of non-sentential utterances in dialogue. School of Informatics, University of Edinburgh dissertation.

Schlenker, Philippe. 2014. Iconic features. Natural Language Semantics, 22(4), 299-356.

Schlenker, Philippe; Aristodemo, Valentina; Ducasse, Ludovic; Lamberton, Jonathan; Santoro, Mirko. 2016. The Unity of Focus: Evidence from Sign Language (ASL and LSF). Linguistic Inquiry 47:363-381.

Schmerling, S. 1975. Asymmetric conjunction and rules of conversation. In P. Cole and J. Morgan (eds.), Speech Acts, Volume 3 of Syntax and Semantics, pp. 211-231. New York: Academic Press.

Schwabe, Kerstin. 2000. Coordinate ellipsis and information structure. In Ellipsis in conjunction. Kerstin Schwabe and Ning Zhang (eds.), 247-269. Tübingen: Niemeyer. 
Schwarz, Bernhard. 2000. Topics in ellipsis. Amherst, MA: University of Massachusetts at Amherst dissertation.

Schwartz, Bonnie D. 1985. Case and Conjunction. Southern California Occasional Papers in Linguistics 10, 161-186.

Selkirk, Elisabeth. 2008. Contrastive focus, givenness and the unmarked status of 'discourse-new', Acta Hungarica Linguistica, 55(3-4), 331-346. Special Issue on Information Structure ed. by Caroline Féry and Gisebert Fanselow.

Siegel, Muffy. 1987. Compositionality, case, and the scope of auxiliaries. Linguistics and philosophy 10:53-76.

Stilo Donald. 2004. Coordination in three Western Iranian languages: Vafsi, Persian and Gilaki. In Haspelmath, Martin (ed.) 2004. Coordinating constructions. (Typological Studies in Language, 58.) Amsterdam: Benjamins.

Schwarzchild, Roger. 1999. GIVENness, AvoidF and other constraints on the placement of accent. Natural Langauge Semantics 7:141-177.

Sportiche, Dominique. 1993. Sketch of a Reductionist Approach to Syntactic Variation and Dependencies. Unpublished manuscript, UCLA.

Stump, Gregory T. 1977. Pseudogapping. Unpublished Ohio State University syntax practicum paper.

Svenonius, Peter. 1992. The extended projection of N: identifying the head of the noun phrase. In Working Papers in Scandinavian Syntax 49, pp. 95-121. 
Tai, J. H.-Y. 1969. Coordination Reduction. Ph.D. thesis, Indiana University.

Tang, Gladys \& Lau, Prudence. 2012. Coordination and subordination. In Roland Pfau, Markus Steinbach \& Bencie Woll (eds.), Sign language: An international handbook, 340365. Berlin: Mouton De Gruyter.

Te Velde, John R. 1996. Coordination and Antisymmetry Theory: Some Evidence from Germanic. American Journal of Germanic Linguistics and Literatures 8.2: 135-175.

1997. Deriving Conjoined XPs: A Minimal Deletion Approach. In Werner Abraham and Elly van Gelderen (eds.), German: Syntactic Problems Problematic Syntax, 231-258, Tübingen: Max Niemeyer.

Toosarvandani, Maziar. 2013. Gapping is low coordination (plus VPellipsis): A reply to johnson. Ms., University of California, Santa Cruz.

2008. Wh-movement and the syntax of sluicing. Journal of Linguistics 44:677-722.

2009. Ellipsis in Farsi complex predicates. Syntax 12:60-92.

Travis, Lisa. 1988. The Syntax of Adverbs. McGill Working Papers in Linguistics: Special Issue on Comparative Germanic Syntax: 280-310

Umbach, Carla. 2001. Contrast and contrastive topic. In KruijfKorbayová, I. \& M. Steedman (eds.), Information Structure, Discourse Structure and Discourse Semantics, 2-13. 
2004. On the Notion of Contrast in Information Structure and Discourse Structure. Journal of Semantics 21:155-175.

2005. Contrast and information structure: A focusbased analysis of 'but'. Linguistics 43, 207-232.

Vallduví, Enric. 1990. "The effects of plasticity in the association of focus and prominence". In Yongkyoon No \& Mark Libucha (eds.), ESCOL '90: Proceedings of the 7th Eastern States Conference on Linguistics. Columbus: Ohio State University Press.

Vallduví, Enric. \& Vilkuna, M. 1998. On rheme and kontrast. In Culicover, P.W. \& L. McNally (eds.), The limits of syntax (Syntax \& Semantics 29), 79-108. San Diego: Academic Press.

Van Gijn, Ingeborg Caren. 2004. The quest for syntactic dependency. Sentential complementation in Sign Language of the Netherlands. Utrecht: LOT.

Van Craenenbroeck, Jeroen \& Merchant, Jason. 2013. Ellipsis phenomena. In den Dikken, Marcel (ed.), The Cambridge Handbook of Generative Syntax, 701-745. Cambridge University Press: Cambridge.

Van Valin Jr, Robert \& LaPolla, Randy .J. 1997. Syntax. Structure, meaning and function. Cambridge: Cambridge University Press.

Velupillai, Viveka. 2012. An introduction to linguistic typology. Amsterdam: John Benjamins. 
Vermeerbergen, Myriam; Leeson, Lorraine; Crasborn, Onno (eds.). 2007. Simultaneity in Sign Language: Form and Function. Amsterdam: John Benjamins.

Vicente, Luis. 2006. Negative short replies in Spanish. In Jeroen van de Weijer and Los (ed.), Linguistics in the Netherlands 2006, 199-211. John Benjamins.

Vicente, Luis. 2010. On the syntax of adversative coordination. Natural Language and Linguistic Theory 28: 381-415.

Vries, Mark de. 2009. On Multidominance and Linearization. Biolinguistics 3, 344-403.

2017. Across-the-Board Phenomena. In Everaert, M. \& H. van Riemsdijk (eds.). The Wiley Blackwell Companion to Syntax, Second Edition, 1-31.

Vos, Mark De. 2005. The Syntax of Pseudo-Coordination in English and Afrikaans, LOT Publications. University of Leiden. Centre for Linguistics.

Warner, Anthony. 1985. The structure of English auxiliaries: A phrase structure grammar. Bloomington, Indiana: Indiana University Linguistics Club.

Wasow, Thomas. 1972. Anaphoric relations in English. Cambridge, MA: MIT dissertation.

Waters, Dafydd \& Sutton-Spence, Rachel. 2005 Connectives in British Sign Language. In: Deaf Worlds 21(3), 129.

Weisser, Philipp. 2015. Derived coordination: A minimalist perspective on clause chains, converbs and asymmetric coordination. Walter de Gruyter GmbH \& Co KG. 
Wilbur, Ronnie B. 1991. Intonation and focus in American Sign Language. In Yongkyoon No \& Mark Libucha (eds.), ESCOL '90: Proceedings of the 7th Eastern States Conference on Linguistics, 320-331. Columbus: Ohio State University Press.

1994. Eyeblinks and ASL Phrase Structure. In: Sign Language Studies 84, 221240.

2000. Phonological and prosodic layering of nonmanuals in American Sign Language. In Emmorey, Karen \& Harlan Lane (eds.), The signs of language revisited, 215244. Mahwah, NJ: Lawrence Erlbaum.

2012. Information structure. In Pfau, Roland \& Markus Steinbach \& Bencie Woll (eds.) Sign language. An international handbook (HSK - Handbooks of linguistics and communication science), 462-489. Berlin: Mouton de Gruyter.

Wilbur, Ronnie B. \& Cynthia Patschke. 1998. Body leans and the marking of contrast in American Sign Language. Journal of Pragmatics 30. 275-303.

Wilder, Chris. 1994. Coordination, ATB and ellipsis. GAGL 37, pp. 291-329.

1996. V2-Effekte: Wortstellungen und Ellipsen. In Deutsch-Typologisch. Institut fur deutsche Sprache.

1997. Some properties of ellipsis in coordination. In A. Alexiadou \& T. Hall (eds.), Studies on Universal Grammar and Typological Variation, 59-107. Amsterdam: John Benjamins. 
2008. Shared constituents and Linearization. In Johnson, Kyle (ed.) , Topics in Ellipsis, 229-258. Cambridge University Press.

Williams, Edwin. 1978. Across the Board Rule Application. Linguistic Inquiry 9: 31-43.

Winkler, Susanne. 2005. Ellipsis and Focus in Generative Grammar. Berlin: Walter de Gruyter.

Zhang, Niina Ning. 2010. Coordination in syntax. Cambridge University Press.

Zwitserlood, Inge. 2012. Classifiers. In R. Pfau, M. Steinbach, \& B. Woll (eds.), Sign Language: an International Handbook (pp. 158-186). Berlin: Mouton de Gruyter.

Zoerner, Ed. 1995. Coordination: The Syntax of \&P. Irvine, CA: University of California dissertation.

Zorzi, Giorgia. 2018. Gapping vs VP-ellipsis in Catalan Sign Language. FEAST 1: 70-81.

Zwart, Jan-Wouter. 2005. Some notes on coordination in head-final languages in Linguistics in the Netherlands 2005, John Benjamins Publishing Company. 